CERN-TH/7216/94

April 1994

\title{
THE THEORY AND PHENOMENOLOGY OF POLARIZED DEEP INELASTIC SCATTERING
}

\author{
M. Anselmino \\ Dipartimento di Fisica Teorica, Università di Torino and Istituto Nazionale di Fisica \\ Nucleare, Sezione di Torino, Via P. Giuria 1, 10125 Torino, Italy. \\ A. Efremov 10 \\ Theory Division, CERN, 1211 Geneva 23, Switzerland. \\ E. Leader \\ Birkbeck College, University of London, Malet Sreet, London WC1E 7HX, UK.
}

To appear in Physics Reports

1) Permanent address: Laboratory of Theoretical Physics, Joint Institute for Nuclear Research, Dubna, 141980, Russia. 


\title{
CONTENTS
}

\section{1) Introduction}

\author{
1.1) Notation
}

2) Polarized lepton-nucleon deep inelastic scattering - general aspects

2.1) General formalism in the one photon exchange approximation

2.1.1) Structure functions and Bjorken scaling

2.1.2) Cross-section differences

2.1.3) Experimental measurement of $g_{1}$ and $g_{2}$ on nucleon targets

a) Longitudinally polarized target

b) Transversely polarized target

c) Combined analysis using both polarizations

d) The problem of extrapolating in $Q^{2}$

2.1.4) Measurement of $g_{1,2}$ on nuclear targets

2.2) Neutral and charged current weak interactions initiated by charged leptons

2.2.1) Weak interaction structure functions and their measurement

3) The Naive Parton Model in polarized DIS

3.1) Projection of $F_{1,2}$ and $g_{1,2}$ from the hadronic tensor $W^{\mu \nu}$

3.2) The hadronic tensor and the nucleon structure functions in the Naive Parton Model

3.3) Intrinsic $\mathbf{p}_{\perp}$ and $g_{2}(x)$

3.3.1) Conflicting Parton Model results for $g_{2}(x)$

3.4) Origin of the difficulties with $g_{2}(x)$

3.5) Weak interaction structure functions in the Naive Parton Model

4) Phenomenological analysis of the data on $g_{1}(x)$ and its first moment $\Gamma_{1}$

4.1) The SLAC-Yale and EMC data: quark distributions near $x=1$

4.2) Analysis of data in the framework of the Operator Product Expansion

4.2.1) Information from hyperon $\beta$-decay

4.2.2) The EMC data on $\Gamma_{1}^{p}$ and its consequences

4.2.3) The EMC data: extrapolation to $x=0$

4.2.4) The EMC data: higher twist effects

a) The Gerasimov, Drell, Hearn approach

b) The QCD sum rule approach

c) Summary on higher twist effects in the EMC experiment

4.3) The EMC result: implications in the Naive Parton Model

4.3.1) The 'Spin crisis in the Parton Model'

4.3.2) The angular momentum sum rule

4.3.3) Trouble with the strange quark

4.4) Analysis of $\Gamma_{1}^{p}$ using the QCD Improved Parton Model

4.4.1) The Operator Product Expansion for $\Gamma_{1}^{p}$

4.5) The new experiments: neutron data and the Bjorken sum rule

4.5.1) The new experiments on deuterium and ${ }^{3} \mathrm{He}$

4.5.2) Tests of the Bjorken sum rule

5) The Operator Product Expansion (OPE)

5.1) General structure of the OPE

5.2) Equations for the moments of $g_{1,2}\left(x, Q^{2}\right)$

$5.3)$ Is there a connection between $g_{1}(x)$ and $g_{2}(x)$ ? 
5.4) Does the first moment of $g_{2}(x)$ vanish? (The Burkhardt-Cottingham sum rule)

5.5) The Efremov-Leader-Teryaev sum rule

6) $g_{1}(x)$ : The axial anomaly and the gluon current $K^{\mu}$

6.1) Is there really a 'spin crisis'?

6.2) On the connection between QCD, the Quark Model and the Naive Parton Model

6.3) The axial anomaly

6.4) The axial gluon current $K^{\mu}$ and the gluon spin

7) $g_{1}(x)$ : Reinterpretation of the measurement of $\Gamma_{1}^{p}$

7.1) The rôle of the anomalous gluon contribution

7.2) Why the gluon contribution survives as $Q^{2} \rightarrow \infty$

7.3) The angular momentum sum rule revisited

8) $g_{1}(x)$ : The anomaly in the QCD Field Theoretic Model

8.1) The factorization theorem

8.2) The gluonic contribution to $g_{1}(x)$ and the definition of $\Delta g(x)$

8.3) Measuring $\Delta g(x)$ via 2-jet production

8.4) The shape of $g_{1}^{p}(x)$ and the anomalous gluon contribution

8.5) The anomaly in $\nu p$ elastic scattering and weak interaction structure functions

8.6) Operator Product Expansion vs. QCD Improved Parton Model

9) $\boldsymbol{g}_{1}(\boldsymbol{x})$ : Non-perturbative effects in the interpretation of the data on $\Gamma_{1}^{p}$

9.1) Topological effects and the axial ghost in QCD: a pedagogical reminder

9.2) A reminder about the $\mathrm{U}(1)$ problem

9.3) The axial ghost

9.4) Rôle of the ghost in the nucleon spin problem

9.5) Attempts to provide a physical interpretation of the EMC result

10) $\boldsymbol{g}_{1,2}(\boldsymbol{x})$ in the QCD Field Theoretic Model

10.1) The QCD Field Theoretic Model

10.2) $g_{1}(x)$ : Longitudinal polarization

10.3) $g_{2}(x)$ : Transverse polarization

10.4) The Naive Parton Model for $g_{2}(x)$ revisited

11) Future Experiments

11.1) Semi-inclusive deep inelastic lepton-hadron reactions

11.2) Hadron-hadron reactions

11.2.1) Hard $\gamma$ and Drell-Yan reactions

11.2.2) Heavy quark production

11.3) Jet handedness

12) Conclusions

Appendices: A) Kinematical relations amongst asymmetries and scaling functions

B) Current matrix elements in the Parton Model

C) Transverse spin: the restoration of electromagnetic gauge invariance

D) Distribution and two-parton correlation functions for transverse spin

E) The Burkhardt-Cottingham and the Efremov-Leader-Teryaev sum rules in the QCD Field Theoretic Model

\section{Acknowledgements}

\section{References}




\section{Introduction}

Deep inelastic lepton-hadron scattering (DIS) has played a seminal role in the development of our present understanding of the sub-structure of elementary particles. The discovery of Bjorken scaling in the late nineteen-sixties provided the critical impetus for the idea that elementary particles contain almost pointlike constituents and for the subsequent invention of the Parton Model. DIS continued to play an essential role in the long period of consolidation that followed, in the gradual linking of partons and quarks, in the discovery of the existence of missing constituents, later identified as gluons, and in the wonderful confluence of all the different parts of the picture into a coherent dynamical theory of quarks and gluons - Quantum Chromodynamics (QCD).

In more recent times the emphasis has shifted to the detailed study of the $x$ dependence of the parton distribution functions and to the study of their $Q^{2}$-evolution, probably the most direct test of the perturbative aspects of QCD.

Polarized DIS, involving the collision of a longitudinally polarized lepton beam on a polarized target (either longitudinally or transversely polarized) provides a different, complementary and equally important insight into the structure of the nucleon. Whereas ordinary DIS probes simply the number density of partons with a fraction $x$ of the momentum of the parent hadron, polarized DIS can partly answer the more sophisticated question as to the number density of partons with given $x$ and given spin polarization, in a hadron of definite polarization, either along or transverse to the motion of the hadron.

But what is quite extraordinary and unexpected ab initio is the richness and subtlety of the dynamical effects associated with the polarized case. Whereas the unpolarized scaling functions $\mathrm{F}_{1,2}(x)$ have a simple interpretation in the Naive Parton Model (where the nucleon is considered as an ensemble of essentially free massless partons) and a straightforward generalisation in the framework of perturbative QCD, the spin dependent scaling functions $g_{1,2}(x)$ are much more subtle, each fascinating in its own way. The function $g_{1}(x)$ which, at first sight, seems trivial to deal with in the Naive Parton Model, turns out, within perturbative QCD, to have an anomalous gluon contribution associated with it. In addition the first moment of $g_{1}(x)$ turns out to be connected with an essentially non-perturbative aspect of $\mathrm{QCD}$, the axial ghost which is invoked to resolve the $U(1)$ problem of the mass of the $\eta^{\prime}$. And $g_{2}(x)$ turns out not to have any interpretation at all in purely partonic language.

What is also fascinating is the extraordinary interplay of theory and experiment in the study of $g_{1}(x)$. For a long time the theory of $g_{1}(x)$ remained comfortably at the level of the Naive Parton Model. Then, in 1988, came the disturbing results of the European Muon Collaboration (EMC) [ASH 88, 89], which differed significantly from the naive theoretical predictions. These results could be argued to imply that the sum of the spins carried by the quarks in a proton $\left(S_{z}^{\text {quarks }}\right)$ was consistent with zero, rather than with $1 / 2$ as given in the quark model, suggesting a "spin crisis in the parton model" [LEA 88]. This led to an intense scrutiny of the basis of the theoretical calculation of $g_{1}(x)$ and the discovery of the anomalous gluon contribution [EFR 88]. (As so often happens in theoretical physics it turns out that such an effect had already been studied to some extent in a largely overlooked paper of 1982 [LAM 82]). So surprising was this discovery that the calculation was immediately checked by three different groups [ALT 88; CAR 88; LEA 88a] who all arrived at the same result. (Somewhat fortuitously, as it turns out, as was demonstrated in [CAR 88]).

In the modified theoretical picture, the quantity $\Delta \Sigma=2 S_{z}^{\text {quark }}$, whose value had to 
be consistent with zero as a consequence of the EMC experiment, is replaced by the linear combination (for 3 flavours) $\Delta \Sigma-\left(3 \alpha_{s} / 2 \pi\right) \Delta g$ where $\Delta g=\int_{0}^{1} d x \Delta g(x)$ and $\Delta g(x)$ is the polarized gluon number density.

It has to be stressed that as a consequence of QCD a measurement of the first moment of $g_{1}(x)$ does not measure the z-component of the sum of the quark spins. It measures only the superposition

$$
\frac{1}{9}\left[\Delta \Sigma-\frac{3 \alpha_{s}}{2 \pi} \Delta g\right]
$$

and this linear combination can be made small by a cancellation between quark and gluon contributions. Thus the EMC results ceases to imply that $\Delta \Sigma$ is small.

The function $g_{2}(x)$, on the other hand, does not have any simple interpretation in the Naive Parton Model and it is a triumph of perturbative QCD that one can derive a sensible, gauge invariant result for it in the QCD Field Theoretic Model [EFR 84].

In this review we concentrate almost exclusively on the polarized case. A good survey of the unpolarized case can be found in [ALT 82] or, at a more introductory level, in [LEA 94]. Our treatment attempts to cover both the theory and the phenomenology of the subject and is meant to be reasonably self-contained.

On the phenomenological side we outline the formalism for discussing DIS in terms of one photon exchange. We briefly mention the extension to weak interactions (both neutral and charged current) initiated by charged leptons. We introduce the scaling functions $g_{1,2}(x)$ and consider how data on them can be extracted from experiments on cross-section asymmetries using both longitudinal and transversely polarized targets. We consider both hydrogen and nuclear targets and we also address the problem of how to extract data at fixed $Q^{2}$ over the whole range of $x$, as needed for the testing of sum rules.

Since the data taken in different $x$-bins correspond to different ranges of $Q^{2}$, the production of a data set for all $x$ at some fixed $Q^{2}$ is a non trivial "experimental" problem. There is some evidence that the Compton asymmetry $A_{1}\left(x, Q^{2}\right)$ is almost independent of $Q^{2}$ and the extraction of $g_{1}\left(x, Q^{2}\right)$ at the desired $Q^{2}$ is carried out by assuming $A_{1}$ independent of $Q^{2}$. Now it is reasonable to believe that $A_{1}\left(x, Q^{2}\right)$ will vary only slowly with $Q^{2}$, but there is no justification for believing it to be exactly constant. Nonetheless it is experimental practice NOT to include an error in $g_{1}\left(x, Q^{2}\right)$ to reflect the uncertainty in the variation of $A_{1}$ with $Q^{2}$. This is, to say the least, quite unjustifiable. A possible strategy for carrying out the extrapolation in $Q^{2}$ is discussed in Section 2.1.3 d).

An extensive phenomenological analysis of all the data on $g_{1}\left(x, Q^{2}\right)$ and its first moment $\Gamma_{1}\left(Q^{2}\right)$ is given including the very recent data from experiments on deuterium and ${ }^{3} \mathrm{He}$ targets. The implications of the data are considered in the framework of the Naive Parton Model and of the Operator Product Expansion (OPE). Aspects discussed include: information from hyperon $\beta$-decay; the extrapolation to $x=0$; higher twist effects.

We argue, in agreement with several recent analyses, that there is absolutely no evidence for a failure of the very fundamental Bjorken sum rule.

On the theoretical side we distinguish between three approaches of varying degrees of sophistication:

i) In the Naive Parton Model the nucleon is viewed as an assembly of free, parallel moving quarks. The treatment is probabilistic and its essence is summarized by the relation connecting the virtual photon-hadron cross-section $\sigma^{\gamma^{*} H}$ to a convolution of the $\gamma^{*}$-quark 
cross-sections and the number density of quarks in the hadron, $f_{q / H}(x)$ :

$$
\sigma^{\gamma^{*} H}=\int d x^{\prime} \sigma^{\gamma^{*} q}\left(x / x^{\prime}\right) f_{q / H}\left(x^{\prime}\right) \text {. }
$$

ii) In the QCD Improved Parton Model one supplements the Naive Parton Model with QCD-controlled, probabilistic, Altarelli-Parisi splitting functions. The description remains probabilistic and the main effect is the replacement of number densities $f_{q / H}(x)$ by $Q^{2}$-dependent densities $f_{q / H}\left(x, Q^{2}\right)$.

iii) In the QCD Field Theoretic Model one starts with genuine QCD Feynman diagrams. We call it a "Model" because the diagrams have to be divided into a hard scattering part, which is calculated strictly according to perturbative QCD, and a soft part which we cannot calculate and which has to be modelled. One makes certain reasonable sounding assumptions about the behaviour of this non-perturbative part. The Model is, in general, not probabilistic and involves hadronic matrix elements of products of quark and gluon interacting fields at different space-time points. In those cases where the result is probabilistic one can usually recover the Naive Parton Model upon treating the fields as free fields, or, with a less drastic approximation, the QCD Improved Parton Model.

The Operator Product Expansion (which deals with matrix elements of local operators), where it can be justified leads to results perfectly compatible with the Field Theoretic Model, but the latter has a much wider field of applicability and can be utilised in situations where use of the OPE cannot be justified.

The calculation of $g_{1,2}(x)$ in the Naive Parton Model is covered in some detail and the source of the difficulties with $g_{2}(x)$ is, we hope, very clearly delineated. We introduce and discuss the general structure of the Operator Product Expansion and give expressions for the n-th moments of $g_{1,2}\left(x, Q^{2}\right)$. We are careful to state the range of $n$ for which the results can be proved and this leads to an examination of various claims in the literature about relationships between $g_{1}$ and $g_{2}$, about the first moment of $g_{2}(x)\left(\int d x g_{2}(x)=0\right.$ : the Burkhardt-Cottingham sum rule) and about the second moment of $g_{1}(x)+2 g_{2}(x)$ $\left(\int d x x\left[g_{1}(x)+2 g_{2}(x)\right]=0\right.$ : the Efremov-Leader-Teryaev sum rule).

We explain that neither of these sum rules follows directly from the OPE and that their validity rests upon an assumption about the invertability of certain Fourier transforms (equivalently, about the behaviour as $x \rightarrow 0$ ) and that this invertability does not occur if $g_{2}(x)$ has the small $x$ behaviour $1 / x^{2}$ suggested by Regge Theory, so that the above integrals diverge.

The axial anomaly and the gluon axial current $K^{\mu}$ are discussed in some detail. We study the connection between $K^{\mu}$ and the gluon spin operator and derive the anomalous gluon contribution to $g_{1}(x)$. There is some disagreement in the literature regarding the kernel relating the polarized gluon number density $\Delta g(x)$ to $g_{1}(x)$, and we give arguments in favour of a particular choice.

There are also arguments in the literature against the whole concept of an anomalous gluon contribution, on the grounds that $K^{\mu}$ is not a gauge invariant operator. Here we would like to remind readers that in gauge theories (even in the Abelian case of QED) it often happens that the operators representing fundamental physical quantities like momentum are not gauge-invariant. This lack of gauge invariance is innocuous and irrelevant provided that the expectation values of those operators between physical states are gauge invariant. Ultimately, the gauge invariance of $\Delta g(x)$ is guaranted by showing how it is related to the physically measurable cross-section for 2-jet production.

There are also claims in the literature that the anomalous contribution to $g_{1}$ vanishes 
if the calculation is carried out with strictly massless gluons. We point out that this is irrelevant. In the QCD Field Theoretic Model there is, in principle, an integral over the $k^{2}$ of the gluon and the point $k^{2}=0$ is of zero measure. Only in a Parton Model calculation with gluon mass fixed at $k^{2}=0$ would there be any problem.

The whole question of the calculation of $g_{1,2}$ and of the anomaly is finally approached from the point of view of the QCD Field Theoretic Model. Contrary to some assertions in the literature we show that there is perfect consistency between this approach and the Operator Product Expansion.

We contrast the calculation of $g_{2}(x)$ and $g_{1}(x)$ in QCD Field Theoretic Model, showing that the Feynman diagrams which have a simple quark-parton interpretation lead to an unacceptable result for $g_{2}(x)$, i.e. a result which does not respect gauge invariance. A detailed demonstration is given of how the unwanted gauge dependent terms are cancelled when one includes diagrams corresponding to quark-gluon correlations in the nucleon, a concept which is outside the framework of the Parton Model.

We provide a brief introduction to non-perturbative and topological effects in QCD, to the $U(1)$ problem, to the axial ghost and to the isosinglet, Generalized GoldbergerTreiman relation (GGT). A connection is established between the total quark spin and the coupling of the $\eta^{\prime}$ to nucleons and this suggests that the sum of the quark spins is, in fact, close to the canonical value of $1 / 2$, in agreement with the interpretation of the EMC data utilising an anonalous gluon contribution to $g_{1}(x)$.

A dominant feature throughout is the polarized gluon distribution $\Delta g(x)$. It is essential that this important element in the internal structure of the nucleon should be measured independently, in as many other reactions as possible. We end with a brief survey of reactions in which this might be feasible.

Finally, a word about notation. It may seem pedantic to squabble about choices of notation. But when a subject threatens to become incomprehensible as a result of an injection of illogical, confusing notation, it is necessary to draw attention to the danger. Our Section on notation, consequently, will comment on this issue.

\section{$1.1 \quad$ Notation}

The Field Theoretic approach to hard scattering was developed in a pioneering paper by Ralston and Soper in 1979 [RAL 79]. They introduced a precise, logical, clear and informative notation for the hadronic matrix elements of the bilocal operators that appear.

On the other hand the spin dependent scaling functions $g_{1,2}(x)$ were introduced by Bjorken in 1966 [BJO 66]. They too have a precise and clear meaning in the expression for the measured spin-dependent cross-section.

A complete relabelling of the Ralston-Soper matrix elements [JAF 91] ought to require serious justification (especially the use of $g_{1}$ for one of them when it is only the leading twist approximation to Bjorken $g_{1}$ ) and such justification has never been offered. We therefore follow the Ralston-Soper notation.

Concerning $\gamma$ matrices and spinors we adopt the conventions of Bjorken and Drell [BJO 65] except that our spinors are normalised so that for a fermion with four-momentum $p^{\mu}=(E, \boldsymbol{p})$

$$
u^{\dagger} u=2 E, \quad v^{\dagger} v=2 E, \quad \bar{u} u=2 M, \quad \bar{v} v=-2 M
$$

for both massive and massless particles. 
One has then

$$
\bar{u}(p) \gamma^{\mu} u(p)=2 p^{\mu} \quad \bar{v}(p) \gamma^{\mu} v(p)=2 p^{\mu}
$$

We use

$$
\gamma_{5}=i \gamma^{0} \gamma^{1} \gamma^{2} \gamma^{3}
$$

and our convariant spin vectors $S^{\mu}$ are normalised to $S_{\mu} S^{\mu}=-1$.

For a fermion of mass $M$, then,

$$
\bar{u}(p, S) \gamma^{\mu} \gamma_{5} u(p, S)=-\bar{v}(p, S) \gamma^{\mu} \gamma_{5} v(p, S)=2 M S^{\mu}
$$

For massless fermions of helicity $\lambda= \pm 1 / 2$, or of chirality $2 \lambda= \pm 1$,

$$
\bar{u}(p, \lambda) \gamma^{\mu} \gamma_{5} u(p, \lambda)=-\bar{v}(p, \lambda) \gamma^{\mu} \gamma_{5} v(\rho, \lambda)=\lim _{M \rightarrow 0} 2 M S^{\mu}(\lambda)=4 \lambda p^{\mu}
$$

All states are normalized so that

$$
\left\langle P^{\prime} \mid P\right\rangle=(2 \pi)^{3} 2 E \delta^{3}\left(\boldsymbol{p}^{\prime}-\boldsymbol{p}\right) .
$$

We use

$$
\varepsilon_{0123}=+1
$$

Finally, because 'transversity' has a quite specific meaning [DAL 56; KOT 70] in terms of a phase convention etc., we avoid its use and simply talk of longitudinal and transverse polarization states.

\section{Polarized lepton-nucleon Deep Inelastic Scattering - General aspect}

\subsection{General formalism in one photon exchange approximation}

Consider the inelastic scattering of polarized leptons on polarized nucleons. We denote by $m$ the lepton mass, $k\left(k^{\prime}\right)$ the initial (final) lepton four-momentum and $s\left(s^{\prime}\right)$ its covariant spin four-vector, such that $s \cdot k=0\left(s^{\prime} \cdot k^{\prime}=0\right)$ and $s \cdot s=-1\left(s^{\prime} \cdot s^{\prime}=-1\right)$; the nucleon mass is $M$ and the nucleon four-momentum and spin four-vector are, respectively, $P$ and $S$. Assuming one photon exchange, see Fig. 2.1, the differential cross-section for detecting the final polarized lepton in the solid angle $d \Omega$ and in the final energy range $\left(E^{\prime}, E^{\prime}+d E^{\prime}\right)$ in the laboratory frame, $P=(M, \mathbf{0}), k=(E, \boldsymbol{k}), k^{\prime}=\left(E^{\prime}, \boldsymbol{k}^{\prime}\right)$, can be written as [LEA 85]

$$
\frac{d^{2} \sigma}{d \Omega d E^{\prime}}=\frac{\alpha^{2}}{2 M q^{4}} \frac{E^{\prime}}{E} L_{\mu \nu} W^{\mu \nu},
$$

where $q=k-k^{\prime}$ and $\alpha$ is the fine structure constant.

In Eq. (2.1.1) the leptonic tensor $L_{\mu \nu}$ is given by

$$
L_{\mu \nu}\left(k, s ; k^{\prime}, s^{\prime}\right)=\left[\bar{u}\left(k^{\prime}, s^{\prime}\right) \gamma_{\mu} u(k, s)\right]^{*}\left[\bar{u}\left(k^{\prime}, s^{\prime}\right) \gamma_{\nu} u(k, s)\right]
$$

and can be split into symmetric $(S)$ and antisymmetric $(A)$ parts under $\mu, \nu$ interchange:

$$
\begin{aligned}
L_{\mu \nu}\left(k, s ; k^{\prime}, s^{\prime}\right) & =L_{\mu \nu}^{(S)}\left(k ; k^{\prime}\right)+i L_{\mu \nu}^{(A)}\left(k, s ; k^{\prime}\right) \\
& +L_{\mu \nu}^{\prime(S)}\left(k, s ; k^{\prime}, s^{\prime}\right)+i L_{\mu \nu}^{\prime(A)}\left(k ; k^{\prime}, s^{\prime}\right)
\end{aligned}
$$


where

$$
\begin{aligned}
L_{\mu \nu}^{(S)}\left(k ; k^{\prime}\right) & =k_{\mu} k_{\nu}^{\prime}+k_{\mu}^{\prime} k_{\nu}-g_{\mu \nu}\left(k \cdot k^{\prime}-m^{2}\right) \\
L_{\mu \nu}^{(A)}\left(k, s ; k^{\prime}\right) & =m \varepsilon_{\mu \nu \alpha \beta} s^{\alpha}\left(k-k^{\prime}\right)^{\beta} \\
L_{\mu \nu}^{\prime(S)}\left(k, s ; k^{\prime}, s^{\prime}\right) & =\left(k \cdot s^{\prime}\right)\left(k_{\mu}^{\prime} s_{\nu}+s_{\mu} k_{\nu}^{\prime}-g_{\mu \nu} k^{\prime} \cdot s\right) \\
& -\left(k \cdot k^{\prime}-m^{2}\right)\left(s_{\mu} s_{\nu}^{\prime}+s_{\mu}^{\prime} s_{\nu}-g_{\mu \nu} s \cdot s^{\prime}\right) \\
& +\left(k^{\prime} \cdot s\right)\left(s_{\mu}^{\prime} k_{\nu}+k_{\mu} s_{\nu}^{\prime}\right)-\left(s \cdot s^{\prime}\right)\left(k_{\mu} k_{\nu}^{\prime}+k_{\mu}^{\prime} k_{\nu}\right) \\
L_{\mu \nu}^{\prime(A)}\left(k ; k^{\prime}, s^{\prime}\right) & =m \varepsilon_{\mu \nu \alpha \beta} s^{\alpha}\left(k-k^{\prime}\right)^{\beta} .
\end{aligned}
$$

On summing Eq. (2.1.3) over $s^{\prime}$ and averaging over $s$ one recovers the usual unpolarized leptonic tensor, $2 L_{\mu \nu}^{(S)}$. On summing over $s^{\prime}$, one obtains $2 L_{\mu \nu}^{(S)}+2 i L_{\mu \nu}^{(A)}$.

The unknown hadronic tensor $W_{\mu \nu}$ is similarly defined in terms of four structure functions as [DRE 64; CAR 72; HEY 72].

$$
W_{\mu \nu}(q ; P, S)=W_{\mu \nu}^{(S)}(q ; P)+i W_{\mu \nu}^{(A)}(q ; P, S)
$$

with

$$
\begin{aligned}
\frac{1}{2 M} W_{\mu \nu}^{(S)}(q ; P) & =\left(-g_{\mu \nu}+\frac{q_{\mu} q_{\nu}}{q^{2}}\right) W_{1}\left(P \cdot q, q^{2}\right) \\
& +\left[\left(P_{\mu}-\frac{P \cdot q}{q^{2}} q_{\mu}\right)\left(P_{\nu}-\frac{P \cdot q}{q^{2}} q_{\nu}\right)\right] \frac{W_{2}\left(P \cdot q, q^{2}\right)}{M^{2}} \\
\frac{1}{2 M} W_{\mu \nu}^{(A)}(q ; P, S) & =\varepsilon_{\mu \nu \alpha \beta} q^{\alpha}\left\{M S^{\beta} G_{1}\left(P \cdot q, q^{2}\right)\right. \\
& \left.+\left[(P \cdot q) S^{\beta}-(S \cdot q) P^{\beta}\right] \frac{G_{2}\left(P \cdot q, q^{2}\right)}{M}\right\} .
\end{aligned}
$$

From Eqs. $(2.1 .1,3,8)$ one has

$$
\begin{aligned}
\frac{d^{2} \sigma}{d \Omega d E^{\prime}} & =\frac{\alpha^{2}}{2 M q^{4}} \frac{E^{\prime}}{E}\left[L_{\mu \nu}^{(S)} W^{\mu \nu(S)}+L_{\mu \nu}^{\prime(S)} W^{\mu \nu(S)}\right. \\
& \left.-L_{\mu \nu}^{(A)} W^{\mu \nu(A)}-L_{\mu \nu}^{\prime(A)} W^{\mu \nu(A)}\right] .
\end{aligned}
$$

The individual terms in square brackets can be separately studied by considering cross-sections or differences between cross-sections with particular initial and final polarizations [ANS 79]. Each of these terms is, at least in principle, a measurable quantity which is either a function of the two spin-averaged structure functions $W_{1}$ and $W_{2}$ (terms containing $W_{\mu \nu}^{(S)}$ ) or of the two spin-dependent structure functions $G_{1}$ and $G_{2}$ (terms containing $\left.W_{\mu \nu}^{(A)}\right)$. For example, the usual unpolarized cross-section is proportional to $L_{\mu \nu}^{(S)} W^{\mu \nu(S)}$

$$
\begin{aligned}
\frac{d^{2} \sigma^{u n p}}{d \Omega d E^{\prime}}\left(k, P ; k^{\prime}\right) & =\frac{1}{4} \sum_{s, s^{\prime}, S} \frac{d^{2} \sigma}{d \Omega d E^{\prime}}\left(k, s, P, S ; k^{\prime}, s^{\prime}\right) \\
& =\frac{\alpha^{2}}{2 M q^{4}} \frac{E^{\prime}}{E} 2 L_{\mu \nu}^{(S)} W^{\mu \nu(S)}
\end{aligned}
$$

while differences of cross-sections with opposite target spins single out the $L_{\mu \nu}^{(A)} W^{\mu \nu(A)}$ term:

$$
\sum_{s^{\prime}}\left[\frac{d^{2} \sigma}{d \Omega d E^{\prime}}\left(k, s, P,-S ; k^{\prime}, s^{\prime}\right)-\frac{d^{2} \sigma}{d \Omega d E^{\prime}}\left(k, s, P, S ; k^{\prime}, s^{\prime}\right)\right]
$$




$$
=\frac{\alpha^{2}}{2 M q^{4}} \frac{E^{\prime}}{E} 4 L_{\mu \nu}^{(A)} W^{\mu \nu(A)}
$$

\subsubsection{Structure functions and Bjorken scaling}

The cross-section for the inelastic scattering of unpolarized leptons on unpolarized

nucleons, in the laboratory frame, can be written explicitely using Eqs. $(2.1 .4,9,12)$ and neglecting the lepton mass, as

$$
\frac{d^{2} \sigma^{u n p}}{d \Omega d E^{\prime}}=\frac{4 \alpha^{2} E^{\prime 2}}{q^{4}}\left[2 W_{1} \sin ^{2} \frac{\theta}{2}+W_{2} \cos ^{2} \frac{\theta}{2}\right]
$$

where $\theta$ is the LAB scattering angle of the lepton. Its measurement supplies information on the unpolarized structure functions $W_{1}\left(P \cdot q, q^{2}\right)$ and $W_{2}\left(P \cdot q, q^{2}\right)$.

In the Bjorken limit, or Deep Inelastic Scattering (DIS) regime,

$$
-q^{2}=Q^{2} \rightarrow \infty \quad \nu=E-E^{\prime} \rightarrow \infty \quad x=\frac{Q^{2}}{2 P \cdot q}=\frac{Q^{2}}{2 M \nu}, \text { fixed }
$$

they are known to approximately scale:

$$
\begin{aligned}
\lim _{B j} M W_{1}\left(P \cdot q, Q^{2}\right) & =F_{1}(x) \\
\lim _{B j} \nu W_{2}\left(P \cdot q, Q^{2}\right) & =F_{2}(x),
\end{aligned}
$$

where $F_{1,2}$ vary very slowly with $Q^{2}$ at fixed $x$.

Similarly, from Eqs. (2.1.5, 10, 13), one has

$$
\begin{gathered}
\sum_{s^{\prime}}\left[\frac{d^{2} \sigma}{d \Omega d E^{\prime}}\left(k, s, P, S ; k^{\prime}, s^{\prime}\right)-\frac{d^{2} \sigma}{d \Omega d E^{\prime}}\left(k, s, P-S ; k^{\prime}, s^{\prime}\right)\right] \equiv \\
\equiv \frac{d^{2} \sigma^{s, S}}{d \Omega d E^{\prime}}-\frac{d^{2} \sigma^{s,-S}}{d \Omega d E^{\prime}}= \\
=\frac{8 m \alpha^{2} E^{\prime}}{q^{4} E}\left\{\left[(q \cdot S)(q \cdot s)+Q^{2}(s \cdot S)\right] M G_{1}+Q^{2}[(s \cdot S)(P \cdot q)-(q \cdot S)(P \cdot s)] \frac{G_{2}}{M}\right\}
\end{gathered}
$$

which yields information on the polarized structure functions $G_{1}\left(P \cdot q, q^{2}\right)$ and $G_{2}\left(P \cdot q, q^{2}\right)$. They also, in the Bjorken limit, are expected to scale approximately:

$$
\begin{aligned}
\lim _{B j} \frac{(P \cdot q)^{2}}{\nu} G_{1}\left(P \cdot q, Q^{2}\right) & =g_{1}(x) \\
\lim _{B j} \nu(P \cdot q) G_{2}\left(P \cdot q, q^{2}\right) & =g_{2}(x) .
\end{aligned}
$$

In terms of $g_{1,2}$ the expression (2.1.10) for $W_{\mu \nu}^{(A)}$ becomes

$$
W_{\mu \nu}^{(A)}(q ; P, s)=\frac{2 M}{P \cdot q} \varepsilon_{\mu v \alpha \beta} q^{\alpha}\left\{S^{\beta} g_{1}\left(x, Q^{2}\right)+\left[S^{\beta}-\frac{(S \cdot q) P^{\beta}}{(P \cdot q)}\right] g_{2}\left(x, Q^{2}\right)\right\} .
$$




\subsubsection{Cross-section differences}

Aiming at gathering information on the polarized structure functions $G_{1}$ and $G_{2}$ we look at Eq. (2.1.17) and specialize it to particular spin configurations of the incoming leptons and target nucleons.

Let us consider first the case of longitudinally polarized leptons, that is initial leptons with spin along $(\rightarrow)$ or opposite $(\leftarrow)$ the direction of motion, while the nucleons at rest are polarized along $(S)$ or opposite $(-S)$ an arbitrary direction $\hat{\boldsymbol{S}}$. We then have

$$
\begin{aligned}
s_{\rightarrow}^{\mu} & =-s_{\leftarrow}^{\mu}=\frac{1}{m}(|\boldsymbol{k}|, \hat{\boldsymbol{k}} E) \quad \hat{\boldsymbol{k}}=\frac{\boldsymbol{k}}{|\boldsymbol{k}|} \\
S^{\mu} & =(0, \hat{\boldsymbol{S}}) .
\end{aligned}
$$

We take the $z$-axis along the incoming lepton direction and define (see Fig. 2.2)

$$
\begin{aligned}
k^{\mu} & =(E, 0,0,|\boldsymbol{k}|) \simeq E(1,0,0,1) \\
k^{\prime \mu} & =\left(E^{\prime}, \boldsymbol{k}^{\prime}\right) \simeq E^{\prime}\left(1, \hat{\boldsymbol{k}}^{\prime}\right) \\
& =E^{\prime}(1, \sin \theta \cos \varphi, \sin \theta \sin \varphi, \cos \theta) \\
\hat{\boldsymbol{S}} & =(\sin \alpha \cos \beta, \sin \alpha \sin \beta, \cos \alpha) .
\end{aligned}
$$

Using Eqs. (2.1.20, 21) into Eq. (2.1.17) one obtains (at leading order in $\mathrm{m} / \mathrm{E}$ )

$$
\begin{aligned}
\frac{d^{2} \sigma^{\rightarrow, S}}{d \Omega d E^{\prime}} & -\frac{d^{2} \sigma^{\rightarrow,-S}}{d \Omega d E^{\prime}}=-\frac{4 \alpha^{2}}{Q^{2}} \frac{E^{\prime}}{E} \\
& \times\left\{\left[E \cos \alpha+E^{\prime} \cos \Theta\right] M G_{1}+2 E E^{\prime}[\cos \Theta-\cos \alpha] G_{2}\right\}
\end{aligned}
$$

$\alpha$ is the polar angle of the nucleon spin direction, i.e. the angle between $\hat{\boldsymbol{k}}$ and $\hat{\boldsymbol{S}}$, and $\Theta$ is the angle between the outgoing lepton direction, $\hat{\boldsymbol{k}}^{\prime}$, and $\hat{\boldsymbol{S}}$ :

$$
\begin{aligned}
\cos \Theta & =\sin \theta \cos \varphi \sin \alpha \cos \beta \\
& +\sin \theta \sin \varphi \sin \alpha \sin \beta+\cos \theta \cos \alpha \\
& =\sin \theta \sin \alpha \cos \phi+\cos \theta \cos \alpha
\end{aligned}
$$

where $\phi=\beta-\varphi$ is the azimuthal angle between the $\left(\hat{\boldsymbol{k}}, \hat{\boldsymbol{k}}^{\prime}\right)$ scattering plane and the $(\hat{\boldsymbol{k}}, \hat{\boldsymbol{S}})$ polarization plane (see Figs. 2.2 and 2.3).

For particular values of $\alpha$ one recovers familiar results [CAR 72; HEY 72]. For nucleons polarized along $(\Rightarrow)$ or opposite $(\Leftarrow)$ the initial lepton direction of motion one has $\alpha=0, \Theta=\theta$, and Eq. (2.1.22) gives

$$
\frac{d^{2} \sigma^{\rightrightarrows}}{d \Omega d E^{\prime}}-\frac{d^{2} \sigma^{\rightleftarrows}}{d \Omega d E^{\prime}}=-\frac{4 \alpha^{2}}{Q^{2}} \frac{E^{\prime}}{E}\left[\left(E+E^{\prime} \cos \theta\right) M G_{1}-Q^{2} G_{2}\right] .
$$

If the nucleons are transversely polarized, that is the nucleon spin is perpendicular to the direction of the incoming lepton, then $\alpha=\pi / 2$ and Eqs. $(2.1 .22,23)$ yield

$$
\frac{d^{2} \sigma^{\rightarrow \Uparrow}}{d \Omega d E^{\prime}}-\frac{d^{2} \sigma^{\rightarrow \Downarrow}}{d \Omega d E^{\prime}}=-\frac{4 \alpha^{2}}{Q^{2}} \frac{E^{\prime 2}}{E} \sin \theta \cos \phi\left(M G_{1}+2 E G_{2}\right) .
$$


Notice that if the nucleon spin is perpendicular to the scattering plane $(\alpha=\phi=$ $\pi / 2)$, then the cross-section difference in Eq. (2.1.25) is zero; such a difference has its maximum absolute value when $\phi=0$ or $\pi$, that is when the nucleon spin vector, perpendicular to $\hat{\boldsymbol{k}}$, lies in the scattering plane.

In the above we considered longitudinally polarized leptons. Note that it is impractical to deal with transversely polarized leptons. Because, for transversely polarized incoming leptons, that is leptons with spin perpendicular to the direction of motion, we have

$$
s=(0, \hat{\boldsymbol{s}}),
$$

with the unit vector $\hat{\boldsymbol{s}}$ orthogonal to $\hat{\boldsymbol{k}}, \hat{\boldsymbol{s}} \cdot \hat{\boldsymbol{k}}=0$. In such a case, contrary to what happens for longitudinally polarized leptons, Eq. (2.1.20), there is no factor $E / m$ to cancel the factor $m / E$ which appears in the cross-section differences (2.1.17), and the latter turn out to be vanishingly small in the large energy limit $(m / E \rightarrow 0)$.

Useful information on the unpolarized structure functions $W_{1}$ and $W_{2}$ could be obtained by looking at initial-final lepton spin asymmetries [ANS 79]; such a technique, however, requires the measurement of the scattered lepton polarization, which is probably too difficult to contemplate at present.

\subsubsection{Experimental measurement of $\boldsymbol{g}_{\mathbf{1}}$ and $\boldsymbol{g}_{\mathbf{2}}$ on nucleon targets}

Measurements of cross-section differences with particular lepton and nucleon spin configurations provide information on the polarized structure functions $G_{1}$ and $G_{2}$ or on the scaling functions $g_{1}$ and $g_{2}$, Eq. (2.1.18).

A single difference measurement, however, only yields information on a combination of $G_{1}$ and $G_{2}$, rather than on the separate structure functions. Extracting from the data values of $G_{1}$ or $G_{2}$ alone still requires some further approximate procedure.

a) Longitudinally polarized target

In the performed experiments [ALG 78; BAU 83, 88; ASH 88, 89; ADE 93, 94; ANT 93] the quantity actually measured is the longitudinal spin-spin asymmetry in $\ell p \rightarrow \ell X$,

$$
A_{\|} \equiv \frac{d \sigma^{\rightleftarrows}-d \sigma^{\rightrightarrows}}{d \sigma^{\rightrightarrows}+d \sigma^{\rightleftarrows}},
$$

where $d \sigma$ is short for $d^{2} \sigma /\left(d \Omega d E^{\prime}\right)$ and the denominator is simply twice the unpolarized cross-sections. From Eqs. (2.1.14) and (2.1.24) we derive

$$
A_{\|}=\frac{Q^{2}\left[\left(E+E^{\prime} \cos \theta\right) M G_{1}-Q^{2} G_{2}\right]}{2 E E^{\prime}\left[2 W_{1} \sin ^{2}(\theta / 2)+W_{2} \cos ^{2}(\theta / 2)\right]} .
$$

For reasons to be explained shortly the asymmetry $A_{\|}$is usually expressed in terms of virtual Compton scattering asymmetries $A_{1,2}$ [LEA 85],

$$
A_{\|}=D\left(A_{1}+\eta A_{2}\right)
$$

where $D$ and $\eta$ are known coefficients given in Appendix A. The analysis of the data then proceeds through subsequent approximate steps, explained in detail in Appendix A, which lead to the expressions

$$
A_{\|} \approx D A_{1}
$$


and

$$
g_{1}(x) \approx \frac{A_{\|}}{D} \frac{F_{2}(x)}{2 x[1+R(x)]},
$$

where $F_{2}(x)$ is the unpolarized scaling structure function, Eq. (2.1.16), and $R$ is the ratio of the longitudinal to transverse cross-section,

$$
R=\frac{W_{2}}{W_{1}}\left(1+\frac{\nu^{2}}{Q^{2}}\right)-1 .
$$

All the approximations involved in the above simplifications can be shown to be quite harmless when measuring $g_{1}[\mathrm{ASH} 88,89]$. Indeed not only is $\eta$ small in $(2.1 .29)$ but one can show that

$$
\left|A_{2}\right| \leq \sqrt{R}
$$

and $R$ is known to be small.

If the goal is simply the knowledge of $g_{1}$ and $g_{2}$ on an event-by-event basis then there is a somewhat more direct way of utilising the data.

From $(2.1 .14,18$ and 28) we have

$$
\frac{M \nu Q^{2} E}{2 \alpha^{2} E^{\prime}\left(E+E^{\prime} \cos \theta\right)} \frac{d^{2} \sigma^{u n p}}{d \Omega d E^{\prime}} A_{\|}=g_{1}-\frac{2 x M}{E+E^{\prime} \cos \theta} g_{2}
$$

which can be written more concisely as

$$
g_{1}-\kappa g_{2}=2 K d \sigma^{u n p} A_{\|}
$$

with

$$
\begin{gathered}
\kappa=\frac{2 x M}{E+E^{\prime} \cos \theta} \approx \frac{x M}{E-Q^{2} /(4 M x)} \\
K=\frac{M \nu Q^{2} E}{4 \alpha^{2} E^{\prime}\left(E+E^{\prime} \cos \theta\right)}=\frac{E E^{\prime} \cos ^{2}(\theta / 2)}{2 x \sigma_{M o t t}\left(E+E^{\prime} \cos \theta\right)}
\end{gathered}
$$

where

$$
\sigma_{\text {Mott }}=\left[\frac{\alpha \cos (\theta / 2)}{2 E \sin ^{2}(\theta / 2)}\right]^{2} .
$$

The RHS of Eq. (2.1.35) is constructed directly from experiment, with no need of further data analyses in order to extract $F_{2}$ and $R$, as required in Eq. (2.1.31).

As we said previously the single measurement of $A_{\|}$(and $d \sigma^{u n p}$ ) gives us information on the combination $g_{1}-\kappa g_{2}$, rather than on $g_{1}$ or $g_{2}$ alone. The usual argument [ALG 78; BAU 83, 88], however, is that the $g_{2}$ term in Eq. (2.1.35) can be safely neglected because of the kinematical coefficient $\kappa$ which, in the large energy limit, is very small, as can be seen from Eqs. (2.1.36). This is indeed confirmed by a more careful analysis of the $g_{2}$ term [LEA 88]. We can thus conclude that the measurement of the quantities on the RHS of Eq. (2.1.35) provides essentially a direct measurement of the polarized scaling structure function $g_{1}$.

If one requires data on $g_{1}\left(x, Q^{2}\right)$ on an event-by-event basis, (2.1.35) is the most direct way to use the experimental data on $A_{\|}$. However, it is of great interest to assemble results for $g_{1}\left(x, Q^{2}\right)$ over the entire $x$-range $0 \leq x \leq 1$ at the same $Q^{2}$. Experimentally this is impossible. The kinematics of the experiment dictates that smaller $x$ will correspond to a smaller range of accessible $Q^{2}$. 
The experimentalist is thus forced to extrapolate the data in $Q^{2}$ at fixed $x$, and the question is then: which quantities vary most smoothly and slowly in $Q^{2}$ ? It is an experimental fact that, where it has been studied, $A_{\|}\left(x, Q^{2}\right) / D$ varies only slowly with $Q^{2}$. (This question is discussed further in Section 4.5). For this reason experimentalists prefer to express their measurements in terms of data on $A_{\|}\left(x, Q^{2}\right)$ via (2.1.31). It is then assumed that $A_{\|}\left(x, Q^{2}\right) / D$ is essentially independent of $Q^{2}$ and the value of $g_{1}\left(x,\left\langle Q^{2}\right\rangle\right)$ quoted, for an experiment with mean $Q^{2}$ equal to $\left\langle Q^{2}\right\rangle$, is really

$$
g_{1}\left(x,\left\langle Q^{2}\right\rangle\right) \equiv\left(\frac{A_{\|}(x)}{D}\right) \frac{F_{2}\left(x,\left\langle Q^{2}\right\rangle\right)}{2 x\left[1+R\left(x,\left\langle Q^{2}\right\rangle\right)\right]} .
$$

It is important to bear this in mind when testing sum rules which hold at fixed $Q^{2}$. A possible strategy for improving the $Q^{2}$ extrapolation [EFR 94a] is discussed in Section 2.1.3 d).

As mentioned above, all the approximations leading to (2.1.31) are safe if one is trying to evaluate $g_{1}(x)$. In the next Section we consider the perpendicular asymmetry $A_{\perp}$ which is used to measure a combination of $g_{1}$ and $g_{2}$ with the aim of extracting $g_{2}$. Since $g_{2}$ is expected to be much smaller than $g_{1}$ some care may be necessary in utilising an approximate version of $g_{1}(x)$.

b) Transversely polarized target

If we want to have information on $g_{2}$ we must then consider other spin-spin asymmetries. By scattering longitudinally polarized leptons on transversely polarized nucleons, one can measure the quantity

$$
A_{\perp} \equiv \frac{d \sigma^{\rightarrow \Downarrow}-d \sigma^{\rightarrow \Uparrow}}{d \sigma^{\rightarrow \Uparrow}+d \sigma^{\rightarrow \Downarrow}}
$$

where, again, $d \sigma$ is short for $d^{2} \sigma /\left(d \Omega d E^{\prime}\right)$ and the denominator is twice the unpolarized cross-section $d \sigma^{u n p}$.

From Eqs. (2.1.14) and (2.1.25) one obtains

$$
A_{\perp}=\frac{Q^{2} \sin \theta\left(M G_{1}+2 E G_{2}\right)}{2 E\left[2 W_{1} \sin ^{2}(\theta / 2)+W_{2} \cos ^{2}(\theta / 2)\right]} \cos \phi
$$

where $\phi$ is the difference between the azimuthal angles of $\hat{\boldsymbol{S}}$ and $\hat{\boldsymbol{k}}^{\prime}, \phi=\beta-\varphi$ (see Figs. 2.2,3). For sake of clarity we show in Fig. 2.4 the spin configuration leading to Eq. (2.1.39), for $\phi=0$. In the following we shall always assume $\phi=0$; if necessary one could integrate Eq. (2.1.39) over the $\phi$ range covered by the experimental apparatus.

One can repeat for $g_{2}$ the same direct procedure followed for $A_{\|}$, Eqs. (2.1.34-36), and can use Eqs. (2.1.14, 18 and 39) (with $\phi=0$ ) to write

$$
g_{2}+\frac{\nu}{2 E} g_{1}=\left(\frac{\nu}{E}\right) K^{\prime} d \sigma^{u n p} A_{\perp}
$$

where

$$
K^{\prime}=\frac{Q^{2} E M \nu}{4 \alpha^{2} E^{\prime 2} \sin \theta}=\frac{E \cos ^{2}(\theta / 2)}{2 x \sigma_{M o t t} \sin \theta}
$$

and $\sigma_{M o t t}$ was given in Eqs. (2.1.36).

A measurement of $A_{\perp}$, i.e. the RHS of (2.1.40), provides direct information on the structure function combination $g_{2}+\nu /(2 E) g_{1}$.

In this case the coefficient of $g_{1}$ is not negligible and to isolate $g_{2}$ one must feed in one's knowledge of $g_{1}$ obtained from the $A_{\|}$measurement. 
The $A_{\perp}$ type experiments are much harder than the $A_{\|}$type so it is perhaps worth noting that one can obtain an estimate of $g_{2}(x)$ from the $A_{\|}$experiments if performed at several different beam energies [LEA 88].

At fixed values of $Q^{2}$ and $x$ the only dependence on the beam energy $E$ in the LHS of (2.1.35) is in the coefficient of $g_{2}(x)$.

A study of the energy variation of the RHS of (2.1.34) thus allows one, in principle, to learn about $g_{2}(x)$. Unfortunately, because the coefficient of $g_{2}(x)$ is so small, it is not clear whether this method is practicable or not.

In a similar way the measurement of $A_{\perp}$ at different beam energies $E$, allows the isolation of $g_{2}(x)$ from (2.1.40). For example, measurements at $E=E_{1}$ and $E_{2}$ yield

$$
\left(E_{1}-E_{2}\right) g_{2}(x)=\left[\begin{array}{ll}
\nu K^{\prime} & d \sigma^{u n p} A_{\perp}
\end{array}\right]_{E=E_{1}}-\left[\nu k^{\prime} d \sigma^{u n p} A_{\perp}\right]_{E=E_{2}} .
$$

Equations $(2.1 .40,41)$ provide the most direct access to $g_{2}$ on an event-by-event basis, provided one's knowledge of $g_{1}$ is accurate enough.

However, as with $g_{1}$, if data is desired at fixed $Q^{2}$ over the entire range of $x$ a different strategy is required which we now describe.

c) Combined analysis using $A_{\|}$and $A_{\perp}$

From the longitudinal polarization data one can extrapolate $A_{\|} / D$ smoothly in $Q^{2}$ at fixed $x$. Combining this with the perpendicular polarization data where it is measured one can construct (see Appendix A for definitions of kinematical coefficients)

$$
A^{\prime} \equiv \frac{\sqrt{Q^{2}}}{2 M} A_{2}=\frac{\sqrt{Q^{2}}}{2 M(1+\xi \eta)}\left\{\xi \frac{A_{\|}}{D}+\frac{A_{\perp}}{d}\right\}
$$

at the values of the $A_{\perp}$ experiment. But $A^{\prime}$ should itself vary slowly with $Q^{2}$ because one has

$$
g_{1}\left(x, Q^{2}\right)+g_{2}\left(x, Q^{2}\right)=\frac{F_{2}\left(x, Q^{2}\right)}{2 x^{2}\left[1+R\left(x, Q^{2}\right)\right]} A^{\prime}\left(x, Q^{2}\right)
$$

so that extrapolation in $Q^{2}$ at fixed $x$ should be smooth. Via (2.1.44) one can thus estimate $g_{1}+g_{2}$ over the entire $x, Q^{2}$ range.

Again, with $A^{\prime}$ extrapolated to the relevant $Q^{2}$, one can obtain an improved evaluation of $g_{1}\left(x, Q^{2}\right)$ via

$$
g_{1}\left(x, Q^{2}\right)=\frac{F_{2}\left(x, Q^{2}\right)}{2 x\left[1+R\left(x, Q^{2}\right)\right]} \cdot \frac{1}{1+4 M^{2} x^{2} / Q^{2}} \cdot\left\{\frac{A_{\|}}{D}-\frac{2 M}{\sqrt{Q^{2}}}\left(\eta-\frac{2 M x}{\sqrt{Q^{2}}}\right) A^{\prime}\right\} .
$$

The formulae (2.1.44) and (2.1.45) are exact. No approximations have been made. And they are expressed in terms of the functions $A^{\prime}\left(x, Q^{2}\right)$ and $A_{\|} / D$ which should both be slowly varying functions of $Q^{2}$ at fixed $x$.

d) The problem of extrapolating in $Q^{2}$

We mentioned in a) above that

$$
A_{1}\left(x, Q^{2}\right)=\frac{A_{\|}\left(x, Q^{2}\right)}{D}
$$

is taken to be independent of $Q^{2}$ in the experimental evaluation of $g_{1}\left(x,\left\langle Q^{2}\right\rangle\right)$ via (2.1.37). There is no allowance made for the error in extrapolating from the measured region of $Q^{2}$ for the $x$ involved to the required value $\left\langle Q^{2}\right\rangle$. A simple linear parametrization $a+b Q^{2}$ or $a+b \ln Q^{2}$ for $A_{1}$ will not work because a best fit will yield a very small value of $b$, but with very large errors, leading to unrealistic error estimates on $g_{1}\left(x,\left\langle Q^{2}\right\rangle\right)$. 
There is no rigorous theoretical solution to this experimental problem, but the following approximate procedure should lead to an improved estimate of $g_{1}\left(x,\left\langle Q^{2}\right\rangle\right)$ and its error [EFR 94a].

Let $x_{1}, x_{2}, \ldots$ be the values of $x$ for the data bins and let $Q_{1}^{2}, Q_{2}^{2}, \ldots$ be the mean values of $Q^{2}$ for each $x$-bin.

We define a zeroth order approximation to $g_{1}\left(x,\left\langle Q^{2}\right\rangle\right)$ for each $x_{i}$ via $(2.1 .37)$, i.e.

$$
g_{1}^{(0)}\left(x_{i},\left\langle Q^{2}\right\rangle\right) \equiv A_{1}\left(x_{i}, Q_{i}^{2}\right) F_{1}\left(x_{i},\left\langle Q^{2}\right\rangle\right)
$$

where we have written

$$
F_{1}=\frac{F_{2}}{2 x[1+R]}
$$

for brevity. We define an improved estimate of $g_{1}\left(x,\left\langle Q^{2}\right\rangle\right)$ for each $x_{i}$ via

$$
g_{1}\left(x_{i},\left\langle Q^{2}\right\rangle\right) \equiv A_{1}\left(x_{i},\left\langle Q^{2}\right\rangle\right) F_{1}\left(x_{i},\left\langle Q^{2}\right\rangle\right)
$$

where $A_{1}\left(x_{i},\left\langle Q^{2}\right\rangle\right)$ is obtained using

$$
A_{1}\left(x_{i},\left\langle Q^{2}\right\rangle\right) \approx A_{1}\left(x_{i}, Q_{i}^{2}\right)+b\left(x_{i}, Q_{i}^{2}\right) \ln \left(\left\langle Q^{2}\right\rangle / Q_{i}^{2}\right)
$$

and $b\left(x_{i}, Q_{i}^{2}\right)$ is estimated from

$$
\begin{aligned}
b\left(x_{i}, Q_{i}^{2}\right) & =\left.\frac{1}{F_{1}\left(x_{i}, Q_{i}^{2}\right)} \frac{\partial g_{1}\left(x_{i}, Q^{2}\right)}{\partial \ln Q^{2}}\right|_{Q^{2}=Q_{i}^{2}} \\
& -\left.\frac{A_{1}\left(x_{i}, Q_{i}^{2}\right)}{F_{1}\left(x_{i}, Q_{i}^{2}\right)} \frac{\partial F_{1}\left(x_{i}, Q^{2}\right)}{\partial \ln Q^{2}}\right|_{Q^{2}=Q_{i}^{2}} .
\end{aligned}
$$

All the terms on the RHS of (2.1.51) are known from experiment except for the derivative of $g_{1}$. The latter can be approximately calculated from the evolution equation provided we use the experimental fact that the flavour singlet part of $g_{1}$ is much smaller than the non-singlet part [see (4.2.22)]. Thus we treat the evolution as if it were purely non-singlet, i.e. we compute

$$
\left.\frac{\partial g_{1}\left(x_{i}, Q^{2}\right)}{\partial \ln Q^{2}}\right|_{Q^{2}=Q_{i}^{2}} \approx \frac{\alpha_{s}\left(Q_{i}^{2}\right)}{2 \pi} \int_{x_{i}}^{1} \frac{d y}{y} g_{1}\left(y, Q_{i}^{2}\right) \Delta P_{q q}\left(\frac{x_{i}}{y}\right),
$$

where $\Delta P_{q q}$ is the non-singlet polarized splitting function. In fact, for the non-singlet case, polarized and unpolarized splitting functions are equal [ALT 77],

$$
\Delta P_{q q}(x)=P_{q q}(x)=\frac{4}{3}\left(\frac{1+x^{2}}{1-x}\right)_{+} .
$$

Finally, in the convolution integral in (2.1.52), we approximate $g_{1}$ by its known zeroth order estimate (2.1.47). Thus we suggest the approximate formula

$$
\left.\frac{\partial g_{1}\left(x_{i}, Q^{2}\right)}{\partial \ln Q^{2}}\right|_{Q^{2}=Q_{i}^{2}} \approx \frac{\alpha_{s}\left(Q_{i}^{2}\right)}{2 \pi} \int_{x_{i}}^{1} \frac{d y}{y} g_{1}^{(0)}\left(y, Q_{i}^{2}\right) \Delta P_{q q}\left(\frac{x_{i}}{y}\right) .
$$

This will, of course, provide an estimate for the value of $b$ in (2.1.51) and for the error on it. 


\subsubsection{Measurement of $\boldsymbol{g}_{1,2}$ on nuclear targets}

We consider spin 1 targets, like deuterium, or spin $1 / 2$ targets like ${ }^{3} \mathrm{He}$. The asymmetries considered are the analogues of (2.1.27 and 38) and are defined for nucleus $A$ by

$$
\begin{aligned}
& A_{\|}^{A}=\frac{d \sigma_{A}^{\stackrel{\vec{\xi}}{\rightleftarrows}}-d \sigma_{A}^{\rightrightarrows}}{d \sigma_{A}^{\rightleftarrows}+d \sigma_{A}^{\rightrightarrows}}
\end{aligned}
$$

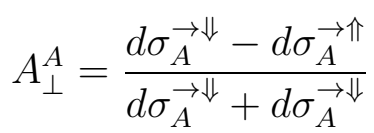

where $\sigma_{A} \vec{A}, \sigma_{A}^{\leftarrow}$ means $J_{z}= \pm 1 / 2$ for a spin $1 / 2$ target, but $J_{z}= \pm 1$ for a spin one target longitudinally polarized; and similarly for transverse polarization.

We deal only with the case where the constituents of the nucleons are assumed to contribute independently to the scattering. This means we neglect shadowing and Fermi motion.

For unpolarized scattering on deuterium this is tantamount to taking

$$
\sigma_{d}=\sigma_{p}+\sigma_{n}
$$

a perfectly reasonable approximation for $\sigma_{d}$ but one which can be misleading when differences of cross-section are being studied. For example the Gottfried sum rule requires the combination $\sigma_{p}-\sigma_{n}$ for which the approximation $2 \sigma_{p}-\sigma_{d}$ may be dangerous because the subtraction of comparable quantities magnifies the error [EPE 93].

In a similar way, for the testing of the Bjorken sum rule we shall require the quantity $g_{1}^{p}-g_{1}^{n}$, and one might worry about obtaining $g_{1}^{n}$ from nuclear data on the basis of independent scattering. Happily because $g_{1}^{p}$ and $g_{1}^{n}$ are expected to be quite different in magnitude this should be quite reliable [EPE 92].

On the basis of independent scattering on the $Z$ protons and $N$ neutrons in the nucleus the nuclear cross-section difference is given by

$$
d \sigma_{A}^{\Rightarrow}-d \sigma_{A}^{\stackrel{\leftarrow}{=}}=2\left[Z d \sigma_{p} \mathcal{P}_{p} A_{\|}^{p}+N d \sigma_{n} \mathcal{P}_{n} A_{\|}^{n}\right]
$$

where $d \sigma_{p, n}$ are the unpolarized nucleon cross-sections, $A_{\|}^{p, n}$ the nucleon longitudinal asymmetries and $\mathcal{P}_{p, n}$ the longitudinal polarization of the nucleons in the nuclear state with $J_{z}=1 / 2$ or 1 .

For the asymmetry defined in (2.1.55) one then has

$$
A_{\|}^{A}=f_{p} \mathcal{P}_{p} A_{\|}^{p}+f_{n} \mathcal{P}_{n} A_{\|}^{n}
$$

where

$$
f_{p}=\frac{Z d \sigma_{p}}{Z d \sigma_{p}+N d \sigma_{n}} \quad f_{n}=\frac{N d \sigma_{n}}{Z d \sigma_{p}+N d \sigma_{n}}
$$

are the fractions of events originating on protons and neutrons respectively.

An analogous result holds for $A_{\perp}^{A}$.

Equation (2.1.59) is the fundamental formula which should be used to extract $A_{\|}^{n}$ from $A_{\|}^{A}$ and a knowledge of $A_{\|}^{p}$. This is especially true when one does not have data on $A_{\|}^{A}$ and $A_{\|}^{p}$ at the same $\left\langle Q^{2}\right\rangle$ because it appears that the asymmetries themselves show very little $Q^{2}$ dependence. 
For deuterium, because of the $D$-state admixture one has

$$
\mathcal{P}_{p}^{d}=\mathcal{P}_{n}^{d}=\left(1-\frac{3}{2} \omega_{D}\right)
$$

where $\omega_{D}=0.058$ is the $D$-state probability.

For the fractions $f_{p, n}$ one has, approximately,

$$
f_{p}^{d}=\frac{F_{2}^{p} /\left(1+R^{p}\right)}{2 F_{2}^{d} /\left(1+R^{d}\right)} \quad f_{n}^{d}=\frac{F_{2}^{n} /\left(1+R^{n}\right)}{2 F_{2}^{d} /\left(1+R^{d}\right)}
$$

where $F_{2}^{d}$ is the deuteron $F_{2}$ per nucleon.

Often one defines $g_{1}^{d}$ per nucleon via $(2.1 .30,31)$

$$
g_{1}^{d}\left(x, Q^{2}\right) \equiv \frac{A_{\|}^{d}}{D} \frac{F_{2}^{d}\left(x, Q^{2}\right)}{2 x\left[1+R^{d}\left(x, Q^{2}\right)\right]}
$$

so that (2.1.59) becomes

$$
g_{1}^{d}\left(x, Q^{2}\right)=\frac{\left(1-\frac{3}{2} \omega_{D}\right)}{2}\left\{g_{1}^{p}\left(x, Q^{2}\right)+g_{1}^{n}\left(x, Q^{2}\right)\right\} .
$$

In the above $(2.1 .55,56)$ we have defined asymmetries for $100 \%$ polarized targets.

For spin $1 / 2$ targets with degree of longitudinal polarization $\mathcal{P}$ the generalization of these equations is quite straightforward:

$$
A_{\|}=\frac{1}{\mathcal{P}}\left\{\frac{d \sigma^{\rightarrow}(-\mathcal{P})-d \sigma^{\rightarrow}(\mathcal{P})}{d \sigma^{\rightarrow}(-\mathcal{P})+d \sigma^{\rightarrow}(\mathcal{P})}\right\}=\frac{1}{\mathcal{P}} \frac{d \sigma^{\rightarrow}(-\mathcal{P})-d \sigma^{\rightarrow}(\mathcal{P})}{2 d \sigma}
$$

where $d \sigma$ is the unpolarized cross-section. A similar result holds for $A_{\perp}$.

For spin 1 targets the result is a little more subtle. If $p_{+}, p_{-}, p_{0}$ are the probabilities of finding states with $J_{z}=1,-1,0$ in the target, then the degree of polarization is [BOU 80]

$$
\mathcal{P}=p_{+}-p_{-}
$$

and the alignment is

$$
\mathcal{A}=1-3 p_{0} .
$$

Then one has

$$
d \sigma^{\rightarrow}(-\mathcal{P})-d \sigma^{\rightarrow}(\mathcal{P})=\mathcal{P}\{d \sigma \stackrel{\rightleftarrows}{\rightleftarrows}-d \sigma \rightrightarrows
$$

but

$$
d \sigma^{\rightarrow}(-\mathcal{P})+d \sigma^{\rightarrow}(\mathcal{P})=2 d \sigma+\frac{\mathcal{A}}{3}\left[d \sigma_{+}+d \sigma_{-}-2 d \sigma_{0}\right]
$$

where $d \sigma$ is the unpolarized cross-section for the spin 1 target.

It follows that for a spin 1 target

$$
A_{\|}=\frac{1}{\mathcal{P}} \frac{d \sigma^{\rightarrow}(-\mathcal{P})-d \sigma^{\rightarrow}(\mathcal{P})}{2 d \sigma+(\mathcal{A} / 3)\left[d \sigma_{+}+d \sigma_{-}-2 d \sigma_{0}\right]}
$$

and this is strictly only equal to

$$
\frac{1}{\mathcal{P}} \frac{d \sigma^{\rightarrow}(-\mathcal{P})-d \sigma^{\rightarrow}(\mathcal{P})}{2 d \sigma}
$$


if the alignment $\mathcal{A}$ is known to be zero.

Of course for the $100 \%$ polarized target corresponding to $(2.1 .55), p_{0}=0$ and the alignment has its maximum value $\mathcal{A}=1$. However, even for $\mathcal{A} \neq 0$, the term in square brackets in $(2.1 .69,70)$ vanishes in the approximation of independent scattering from the nucleons. Since the results $(2.1 .59,60)$ are based on this approximation it is consistent in these equations to ignore the term proportional to $\mathcal{A}$.

It is worth noting that one could, in principle, check the assumption of independent scattering by experimentally testing (2.1.69).

For ${ }^{3} \mathrm{He}$ the wave-function is almost entirely an $S$-state with the two protons having opposite spins. Thus all the spin is carried by the neutron but there is some mixing in the wave-function [WOL 89; CIO 93] and one estimates

$$
\mathcal{P}_{n}^{3} \mathrm{He}=(87 \pm 2) \% \quad \mathcal{P}_{p}^{3} \mathrm{He}=(-2.5 \pm 0.3) \%
$$

For the fractions $f_{p, n}$ one takes the approximation

$$
\begin{aligned}
f_{n}^{3} \mathrm{He} & =\frac{F_{2}^{n} /\left(1+R^{n}\right)}{3 F_{2}^{3} \mathrm{He} /\left(1+R^{3} \mathrm{He}\right)} \\
f_{p}^{3} \mathrm{He} & =\frac{2 F_{2}^{p} /\left(1+R^{p}\right)}{3 F_{2}^{3} \mathrm{He} /\left(1+R^{3} \mathrm{He}\right)}
\end{aligned}
$$

where $F_{2}^{3} \mathrm{He}$ is the helium-3 $F_{2}$ per nucleon.

Defining $g_{1}^{3} \mathrm{He}$ per nucleon via (2.1.31),

$$
g_{1}^{3} \mathrm{He}\left(x, Q^{2}\right)=\frac{A_{\|}^{3} \mathrm{He}}{D} \frac{F_{2}^{3} \mathrm{He}\left(x, Q^{2}\right)}{2 x\left[1+R^{3} \mathrm{He}\left(x, Q^{2}\right)\right]},
$$

one obtains

$$
g_{1}^{3} \mathrm{He}\left(x, Q^{2}\right)=\frac{1}{3}\left[(0.87 \pm 0.02) g_{1}^{n}\left(x, Q^{2}\right)-(0.050 \pm 0.006) g_{1}^{p}\left(x, Q^{2}\right)\right] .
$$

Note that the above is again based on completely independent scattering from the constituent nucleons. This might be reasonable at high $Q^{2}$ but it is not likely to be a good approximation in the E142 experiment which has $\left\langle Q^{2}\right\rangle=2(\mathrm{GeV} / \mathrm{c})^{2}$ and which will be discussed in Section 4.5. Unfortunately we do not have any simple prescription for improving the analysis.

\subsection{Neutral and charged current weak interactions initiated by charged leptons}

Consider now the deep inelastic scattering of longitudinally polarized leptons on polarized nucleons at very high energies, taking into account weak interactions of both the neutral and charged current type. We do not study neutrino initiated processes, although the extension of our results to such a case would be trivial, because of the technical difficulties in polarizing the large nucleon targets needed for neutrino scattering, which make such experiments quite unrealistic. Useful information can be found in [NAS 71; DER 73; NIK 73; AHM 76; KAU 77; JOS 77; CAH 78; BAR 79; CRA 83; LAM 89; VOG 91; JEN 91; RAV 92; MAT 92, 92b; XIA 93; DEF 93]. 
Let us start from neutral current processes, $\ell N \rightarrow \ell X$, which, at lowest perturbative order, proceed via the exchange of one photon or one $Z^{0}$ boson, Figs. 2.1, 5a. The crosssection then receives contributions from a purely electromagnetic, a purely weak and an interference term, so that Eq. (2.1.1) becomes

$$
\frac{d^{2} \sigma_{n c}}{d \Omega d E^{\prime}}=\frac{\alpha^{2}}{2 M q^{4}} \frac{E^{\prime}}{E} \sum_{i=\gamma, \gamma Z, Z} L_{\mu \nu}^{i} W_{i}^{\mu \nu} \eta^{i} .
$$

On summing over the final lepton spins and neglecting terms proportional to $m / E$ or $m / E^{\prime}$, the leptonic tensors $L_{\mu \nu}^{i}$ are given, for negatively charged leptons $\left(e^{-}, \mu^{-}\right)$, by

$$
\begin{aligned}
L_{\mu \nu}^{\gamma} & =\sum_{s^{\prime}}\left[\bar{u}\left(k^{\prime}, s^{\prime}\right) \gamma_{\mu} u(k, \lambda)\right]^{*}\left[\bar{u}\left(k^{\prime}, s^{\prime}\right) \gamma_{\nu} u(k, \lambda)\right] \\
& =2\left[k_{\mu} k_{\nu}^{\prime}+k_{\mu}^{\prime} k_{\nu}-k \cdot k^{\prime} g_{\mu \nu}-2 i \lambda \varepsilon_{\mu \nu \alpha \beta} k^{\alpha} k^{\prime \beta}\right] \\
L_{\mu \nu}^{\gamma Z} & =\sum_{s^{\prime}}\left[\bar{u}\left(k^{\prime}, s^{\prime}\right) \gamma_{\mu}\left(g_{V}-g_{A} \gamma_{5}\right) u(k, \lambda)\right]^{*}\left[\bar{u}\left(k^{\prime}, s^{\prime}\right) \gamma_{\nu} u(k, \lambda)\right] \\
& =\left(g_{V}-2 \lambda g_{A}\right) L_{\mu \nu}^{\gamma} \\
L_{\mu \nu}^{Z} & =\sum_{s^{\prime}}\left[\bar{u}\left(k^{\prime}, s^{\prime}\right) \gamma_{\mu}\left(g_{V}-g_{A} \gamma_{5}\right) u(k, \lambda)\right]^{*}\left[\bar{u}\left(k^{\prime}, s^{\prime}\right) \gamma_{\nu}\left(g_{V}-g_{A} \gamma_{5}\right) u(k, \lambda)\right] \\
& =\left(g_{V}-2 \lambda g_{A}\right)^{2} L_{\mu \nu}^{\gamma}
\end{aligned}
$$

where $2 \lambda= \pm 1$ denotes twice the helicity of the initial lepton. $L_{\mu \nu}^{\gamma}$ agrees with Eqs. (2.1.35 ), upon remembering that for a fast moving lepton with helicity $\lambda$ one has $s^{\mu} \approx 2 \lambda k^{\mu} / \mathrm{m}$. For positively charged leptons $\left(e^{+}, \mu^{+}\right)$one should simply replace, in Eqs. $(2.2 .3,4), g_{A}$ by $-g_{A}$. In our notation

$$
g_{V}=-\frac{1}{2}+2 \sin ^{2} \theta_{W} \quad g_{A}=-\frac{1}{2} .
$$

The factors $\eta^{i}$ in Eq. (2.2.1) collect some kinematical factors, coupling constants and the relative weights of different propagators, namely:

$$
\begin{aligned}
\eta^{\gamma} & =1 \\
\eta^{\gamma Z} & =\left(\frac{G M_{Z}^{2}}{2 \sqrt{2} \pi \alpha}\right)\left(\frac{Q^{2}}{Q^{2}+M_{Z}^{2}}\right) \\
\eta^{Z} & =\left(\eta^{\gamma Z}\right)^{2}
\end{aligned}
$$

where $G$ is the Fermi coupling constant and $M_{Z}$ is the $Z_{0}$ mass. Notice that $G M_{Z}^{2} /(2 \sqrt{2} \pi \alpha)$ $=\left(4 \sin ^{2} \theta_{W} \cos ^{2} \theta_{W}\right)^{-1} \simeq 4 / 3$.

Finally the hadronic tensor $W_{i}^{\mu \nu}$ defines the coupling of the electro-weak current to the nucleon; exploiting Lorentz and CP invariance it can be expressed in terms of 8 independent structure functions as [BAR 79]

$$
\begin{aligned}
\frac{1}{2 M} W_{\mu \nu}^{i} & =-\frac{g_{\mu \nu}}{M} F_{1}^{i}+\frac{P_{\mu} P_{\nu}}{M(P \cdot q)} F_{2}^{i} \\
& +i \frac{\varepsilon_{\mu \nu \alpha \beta}}{2 P \cdot q}\left[\frac{P^{\alpha} q^{\beta}}{M} F_{3}^{i}+2 q^{\alpha} S^{\beta} g_{1}^{i}-4 x P^{\alpha} S^{\beta} g_{2}^{i}\right] \\
& -\frac{P_{\mu} S_{\nu}+S_{\mu} P_{\nu}}{2 P \cdot q} g_{3}^{i}+\frac{S \cdot q}{(P \cdot q)^{2}} P_{\mu} P_{\nu} g_{4}^{i}+\frac{S \cdot q}{P \cdot q} g_{\mu \nu} g_{5}^{i}
\end{aligned}
$$


Notice that terms proportional to $q^{\mu}$ or $q^{\nu}$ can be dropped in the definition of $W_{\mu \nu}^{i}$ because they give no contribution (in the $m / E \rightarrow 0$ limit) when contracted with $L_{i}^{\mu \nu}$. Other definitions of the hadronic tensor appearing in the literature differ from ours because of these terms. In particular the coefficient of $g_{2}$ could be written in a more familiar way using the identity [HEI 73]

$$
\begin{aligned}
\varepsilon_{\mu \nu \alpha \beta} P^{\alpha} S^{\beta} & =\frac{\varepsilon_{\mu \nu \alpha \beta}}{2 x P \cdot q}\left[(q \cdot S) q^{\alpha} P^{\beta}-(P \cdot q) q^{\alpha} S^{\beta}\right] \\
& -\left(q_{\mu} \varepsilon_{\nu \alpha \beta \gamma}-q_{\nu} \varepsilon_{\mu \alpha \beta \gamma}\right) \frac{P^{\alpha} q^{\beta} S^{\gamma}}{2 x P \cdot q}
\end{aligned}
$$

and dropping the last two terms.

The structure functions $F_{j}^{i}\left(P \cdot q, q^{2}\right)$ and $g_{j}^{i}\left(P \cdot q, q^{2}\right)$ are expected to scale approximately in the Bjorken limit and to depend only on $x=Q^{2} /(2 P \cdot q)$. The $F_{j}^{i}$ are the unpolarized structure functions and the $g_{j}^{i}$ the polarized ones: when averaging over the nucleon spin one sums $W_{\mu \nu}(P, q, S)$ to $W_{\mu \nu}(P, q,-S)$ and all terms proportional to $g_{j}^{i}$ cancel out. In Eq. (2.2.7) we have allowed for parity violation and indeed $W_{\mu \nu}^{i}$ is a mixture of second rank tensors and pseudotensors. In case of pure electromagnetic interactions $(i=\gamma)$ parity is conserved and one has

$$
F_{3}^{\gamma}=g_{3}^{\gamma}=g_{4}^{\gamma}=g_{5}^{\gamma}=0 .
$$

In this case Eq. (2.2.7), upon using Eq. (2.2.8) and up to irrelevant terms proportional to $q^{\mu}$ or $q^{\nu}$, reproduces Eqs. $(2.1 .8-10,16,18) . F_{3}, g_{3}, g_{4}$ and $g_{5}$ only contribute to parity violating interactions and are often referred to as the parity violating structure functions.

From Eqs. (2.2.1-4) and $(2.2 .6,7)$ one obtains explicit expressions of the crosssections; some of them can be found in [ANS 93].

In case of charged current interactions, $\ell^{\mp} N \rightarrow \nu(\bar{\nu}) X$, the process is, at leading order, mediated by the exchange of a $W$ meson and it resembles the $Z_{0}$ contribution to the neutral current process, with the assignments $g_{V}=g_{A}=1$. In fact Eq. (2.2.1) now reads

$$
\frac{d^{2} \sigma_{c c}}{d \Omega d E^{\prime}}=\frac{\alpha^{2}}{2 M q^{4}} \frac{E^{\prime}}{E} L_{\mu \nu}^{W} W_{W}^{\mu \nu} \eta^{W}
$$

with $W_{W}^{\mu \nu}$ given by Eq. (2.2.7) in which the label $i$ is now $W$. For a negatively charged lepton $\ell^{-}$(which couples to a $W^{-}$),

$$
\begin{aligned}
L_{\mu \nu}^{W^{-}} & =\sum_{s^{\prime}}\left[\bar{u}\left(k^{\prime}, s^{\prime}\right) \gamma_{\mu}\left(1-\gamma_{5}\right) u(k, \lambda)\right]^{*}\left[\bar{u}\left(k^{\prime}, s^{\prime}\right) \gamma_{\nu}\left(1-\gamma_{5}\right) u(k, \lambda)\right] \\
& =(1-2 \lambda)^{2} L_{\mu \nu}^{\gamma} .
\end{aligned}
$$

Equation (2.2.11) shows that fast $\ell^{-}$leptons only couple to a $W$ if they have a negative helicity $(\lambda=-1 / 2)$. Equation (2.2.10) is completed by

$$
\eta^{W}=\frac{1}{2}\left(\frac{G M_{W}^{2}}{4 \pi \alpha} \frac{Q^{2}}{Q^{2}+M_{W}^{2}}\right)^{2}
$$

where $M_{W}$ is the $W$ mass. For a positively charged lepton $\ell^{+}$one simply changes, as usual, the sign of the axial coupling $\gamma^{\mu} \gamma^{5}$, that is one replaces $\lambda$ with $-\lambda$ in Eq. (2.2.11) to obtain $L_{\mu \nu}^{W^{+}}=(1+2 \lambda)^{2} L_{\mu \nu}^{\gamma}$. 


\subsubsection{Weak interaction structure functions and their measurement}

In order to obtain information on the polarized structure functions observed in weak interaction deep inelastic scattering we should study, in analogy to Eq. (2.1.22), differences of cross-sections with opposite nucleon spin. Rather than considering here the most general case, which could be derived from Eqs. (2.2.1-7) or (2.2.10-12) and (2.1.21), we look at particular nucleon spin configurations, namely longitudinal or transverse ones, like in Eqs. (2.1.24) and (2.1.25) respectively.

To do so we switch to the more convenient variables $x$ and $y \equiv \nu / E$, using

$$
\frac{d^{2} \sigma}{d \Omega d E^{\prime}}=\frac{E^{\prime}}{y M E} \frac{d^{3} \sigma}{d x d y d \varphi} .
$$

We then notice that in the high energy region one can neglect terms proportional to $M / E$ and that, from Eq. (2.2.6) and for $Q^{2}$ values up to $\sim 10^{3} \mathrm{GeV}^{2}$, one has

$$
\eta^{Z} \ll \eta^{\gamma Z} \ll \eta^{\gamma}
$$

Taking all this into account and adopting the same symbols to denote the nucleon spin as in Eqs. (2.1.24) and (2.1.25), one obtains for neutral current reactions:

$$
\begin{gathered}
\frac{d^{2} \sigma_{n c}^{\rightrightarrows}}{d x d y}-\frac{d^{2} \sigma_{n c}}{d x d y} \approx-16 \pi M E \frac{\alpha^{2}}{Q^{4}} x y(2-y) g_{1}^{\gamma} \\
\frac{d^{2} \sigma_{n c} \rightarrow}{d x d y d \varphi}-\frac{d^{2} \sigma_{n c}^{\rightarrow \Downarrow}}{d x d y d \varphi} x \approx-8 M \frac{\alpha^{2}}{Q^{4}} \cos \phi \sqrt{2 x y(1-y) M E} x\left[y g_{1}^{\gamma}+2 g_{2}^{\gamma}\right]
\end{gathered}
$$

and for charged current reactions initiated by $\ell^{\mp}$

$$
\begin{aligned}
\frac{d^{2} \sigma_{c c}^{\rightrightarrows}}{d x d y}-\frac{d^{2} \sigma_{c c}^{\rightleftarrows}}{d x d y} & \approx 64 \pi M E \frac{\alpha^{2}}{Q^{4}} \eta^{W} \\
& \times\left[ \pm x y(2-y) g_{1}^{W^{\mp}}+(1-y)\left(g_{3}^{W^{\mp}}-g_{4}^{W^{\mp}}\right)+x y^{2} g_{5}^{W \mp}\right] \\
\frac{d^{3} \sigma_{c c}^{\rightarrow \Uparrow}}{d x d y d \varphi}-\frac{d^{3} \sigma_{c c}^{\rightarrow \Downarrow}}{d x d y d \varphi} & \approx 32 M \frac{\alpha^{2}}{Q^{4}} \eta^{W} \cos \phi \sqrt{2 x y(1-y) M E} \\
& \times\left[ \pm x y g_{1}^{W^{\mp}} \pm 2 x g_{2}^{W^{\mp}}+\frac{1}{2} g_{3}^{W^{\mp}}+\frac{1-y}{y} g_{4}^{W^{\mp}}-x y g_{5}^{W \mp}\right] .
\end{aligned}
$$

Equations $(2.2 .15,16)$ show that in our kinematical range the neutral current processes are still dominated by one photon exchange and thus agree with Eqs. $(2.1 .24,25)$ respectively. The charged current cross-sections instead, although much smaller than the neutral current ones due to the factor $\eta^{W}$, Eq. (2.2.12), depend also on the parity violating polarized structure functions $g_{3,4,5}^{W^{\mp}}$. (Recall that the \pm signs and the $W^{\mp}$ labels in Eqs. $(2.2 .17,18)$ refer to negatively or positively charged leptons, $\ell^{\mp}$.)

One could also extract information on the parity violating structure functions in neutral current processes by looking at appropriate combinations of cross-sections [ANS 93]. Ideally, it would be very helpful to perform large $Q^{2}$ experiments with both $\ell^{-}$and 
$\ell^{+}$leptons with positive $(\rightarrow)$ and negative $(\leftarrow)$ helicities. In fact, always within the $Q^{2}$ range of validity of Eq. (2.2.14) and noticing, from Eq. (2.2.5), that $g_{V} \simeq 0.04$ whereas $g_{A}=-0.5$, one has:

$$
\begin{aligned}
& \left(\frac{d^{2} \underset{\sigma_{n c}}{\Rightarrow}}{d x d y}-\frac{d^{2} \underset{\sigma_{n c}}{\stackrel{\rightleftarrows}{\rightleftarrows}}}{d x d y}\right)_{\ell^{-}}+\left(\frac{d^{2} \sigma_{n c}^{\rightrightarrows}}{d x d y}-\frac{d^{2} \sigma_{n c}^{\rightleftarrows}}{d x d y}\right)_{\ell^{+}} \\
& +\left(\frac{d^{2} \sigma_{n c}^{\leftrightarrows}}{d x d y}-\frac{d^{2} \sigma_{n c}^{\overleftarrow{\digamma}}}{d x d y}\right)_{\ell^{-}}+\left(\frac{d^{2} \sigma_{n c}}{d x d y}-\frac{d^{2} \sigma_{n c}^{\overleftarrow{\digamma}}}{d x d y}\right)_{\ell^{+}} \approx \\
& \approx 64 \pi M E \frac{\alpha^{2}}{Q^{4}}\left\{(1-y)\left[g_{V} \eta^{\gamma Z}\left(g_{3}^{\gamma Z}-g_{4}^{\gamma Z}\right)+g_{A}^{2} \eta^{Z}\left(g_{3}^{Z}-g_{4}^{Z}\right)\right]\right. \\
& \left.+x y^{2}\left[g_{V} \eta^{\gamma Z} g_{5}^{\gamma Z}+g_{A}^{2} \eta^{Z} g_{5}^{Z}\right]\right\} \\
& \left(\frac{d^{2} \sigma_{n c}^{\rightrightarrows}}{d x d y}-\frac{d^{2} \sigma_{n c}^{\rightleftarrows}}{d x d y}\right)_{\ell^{-}}-\left(\frac{d^{2} \sigma_{n c}}{d x d y}-\frac{d^{2} \sigma_{n c}^{\rightleftarrows}}{d x d y}\right)_{\ell^{+}} \\
& +\left(\frac{d^{2} \sigma_{n c}}{d x d y}-\frac{d^{2} \sigma_{n c}^{\overleftarrow{\digamma}}}{d x d y}\right)_{\ell^{-}}-\left(\frac{d^{2} \sigma_{n c}}{d x d y}-\frac{d^{2} \sigma_{n c}^{\overleftarrow{\digamma}}}{d x d y}\right)_{\ell^{+}} \approx \\
& \approx 64 \pi M E \frac{\alpha^{2}}{Q^{4}} x y(2-y) g_{A} \eta^{\gamma Z} g_{1}^{\gamma Z} \\
& \left(\frac{d^{2} \sigma_{n c}}{d x d y}-\frac{d^{2} \sigma_{n c}^{\rightleftarrows}}{d x d y}\right)_{\ell^{-}}-\left(\frac{d^{2} \sigma_{n c}}{d x d y}-\frac{d^{2} \sigma_{n c}^{\rightleftarrows}}{d x d y}\right)_{\ell^{+}} \\
& -\left(\frac{d^{2} \sigma_{n c}^{\leftrightarrows}}{d x d y}-\frac{d^{2} \sigma_{n c}^{\leftrightarrows}}{d x d y}\right)_{\ell^{-}}+\left(\frac{d^{2} \sigma_{n c}}{d x d y}-\frac{d^{2} \sigma_{n c}^{\overleftarrow{\rightleftarrows}}}{d x d y}\right)_{\ell^{+}} \approx \\
& \approx-64 \pi M E \frac{\alpha^{2}}{Q^{4}}\left\{(1-y) g_{A} \eta^{\gamma Z}\left(g_{3}^{\gamma Z}-g_{4}^{\gamma Z}\right)+x y^{2} g_{A} \eta^{\gamma Z} g_{5}^{\gamma Z}\right\}
\end{aligned}
$$

where $\ell^{-}$and $\ell^{+}$label negative and positive charge leptons respectively. Other interesting combinations of cross-sections can be found in [ANS 93]. Notice that in Eqs. (2.2.19 and 20) a sum over the $\ell^{-}$and $\ell^{+}$helicities is performed, which amounts to considering unpolarized leptons.

In case only $\ell^{-}$unpolarized lepton beams are available one can still obtain information on the parity violating polarized structure functions, by scattering the leptons off longitudinally polarized nucleons and measuring [BIL 75]:

$$
\frac{1}{2}\left(\frac{d^{2} \sigma_{n c}^{\rightrightarrows}}{d x d y}-\frac{d^{2} \sigma_{n c}^{\rightleftarrows}}{d x d y}\right)+\frac{1}{2}\left(\frac{d^{2} \sigma_{n c}}{d x d y}-\frac{d^{2} \sigma_{n c}^{\rightleftarrows}}{d x d y}\right) \equiv \frac{d^{2} \sigma^{\Rightarrow}}{d x d y}-\frac{d^{2} \sigma^{\Leftarrow}}{d x d y}
$$




$$
\begin{aligned}
& \approx 16 \pi M E \frac{\alpha^{2}}{Q^{4}}\left\{(1-y)\left[g_{V} \eta^{\gamma Z}\left(g_{3}^{\gamma Z}-g_{4}^{\gamma Z}\right)+g_{A}^{2} \eta^{Z}\left(g_{3}^{Z}-g_{4}^{Z}\right)\right]\right. \\
& \left.+x y^{2}\left[g_{V} \eta^{\gamma Z} g_{5}^{\gamma Z}+g_{A}^{2} \eta^{Z} g_{5}^{Z}\right]+x y(2-y) g_{A} \eta^{\gamma Z} g_{1}^{\gamma Z}\right\} .
\end{aligned}
$$

Eq. (2.2.22) holds also for unpolarized $\ell^{+}$leptons, provided $g_{A}$ is replaced by $-g_{A}$, so that on summing and subtracting the $\ell^{-}$and the $\ell^{+}$contributions one recovers respectively Eqs. (2.2.19) and (2.2.20). Of course, the above measurements (2.2.19-22) are difficult ones in that they single out non leading parts of the cross-sections. However, they may be feasable and would provide further information on the structure of the nucleon.

\section{The Naive Parton Model in polarized DIS}

\subsection{Projection of $F_{1}, F_{2}, g_{1}$ and $g_{2}$ from the hadronic tensor $W_{\mu \nu}$}

In Section 2 we have written the most general hadronic tensor $W_{\mu \nu}$, Eqs. (2.1.8-10), which describes the unknown coupling of the virtual photon to the composite nucleon in terms of the four structure functions $F_{1}=M W_{1}, F_{2}=\nu W_{2}, g_{1}=M^{2} \nu G_{1}$ and $g_{2}=M \nu^{2} G_{2}$; in the large $Q^{2}$ Bjorken limit, Eqs. $(2.1 .16,18)$, these are supposed to scale, that is to depend only on the Bjorken variable $x=Q^{2} / 2 M \nu$. By performing DIS experiments one learns about the structure functions.

The hadronic tensor $W_{\mu \nu}$ can also be computed from models of the nucleon, as we shall soon illustrate using the Parton Model. In such a case it is convenient to have a technique which easily allows one to extract the four different structure functions from a knowledge of $W_{\mu \nu}(N)$.

Let us start from the unpolarized case. Defining

$$
\begin{aligned}
& P_{1}^{\alpha \beta} \equiv \frac{1}{4}\left[\frac{1}{a} P^{\alpha} P^{\beta}-g^{\alpha \beta}\right] \\
& P_{2}^{\alpha \beta} \equiv \frac{3 P \cdot q}{4 a}\left[\frac{P^{\alpha} P^{\beta}}{a}-\frac{1}{3} g^{\alpha \beta}\right]
\end{aligned}
$$

with

$$
a=\frac{P \cdot q}{2 x}+M^{2}
$$

one can see, from Eqs. (3.1.1-3) and (2.1.8-10), that

$$
P_{1}^{\alpha \beta} W_{\alpha \beta}(N)=F_{1}
$$

and

$$
P_{2}^{\alpha \beta} W_{\alpha \beta}(N)=F_{2}
$$

Similarly, in the polarized case one can introduce the projectors

$$
\begin{aligned}
P_{3}^{\alpha \beta} & \equiv \frac{(P \cdot q)^{2}}{b M^{2}(q \cdot S)}\left[(q \cdot S) S_{\lambda}+q_{\lambda}\right] P_{\eta} \varepsilon^{\alpha \beta \lambda \eta} \\
P_{4}^{\alpha \beta} & \equiv \frac{1}{b}\left\{\left[\frac{(P \cdot q)^{2}}{M^{2}}+2(P \cdot q) x\right] S_{\lambda}+(q \cdot S) q_{\lambda}\right\} P_{\eta} \varepsilon^{\alpha \beta \lambda \eta}
\end{aligned}
$$

with

$$
b=-4 M\left[\frac{(P \cdot q)^{2}}{M^{2}}+2(P \cdot q) x-(q \cdot S)^{2}\right]
$$


such that, from Eqs. (3.1.6-8) and (2.1.8-10),

$$
\begin{aligned}
P_{3}^{\alpha \beta} W_{\alpha \beta}(N) & =g_{2} \\
P_{4}^{\alpha \beta} W_{\alpha \beta}(N) & =g_{1}+g_{2} .
\end{aligned}
$$

Equations (3.1.1-10) can be used in any reference frame and for arbitrary directions of the nucleon spin four-vector; however, some care must be taken in particular cases in which a limiting procedure is required.

\subsection{The hadronic tensor and the nucleon structure functions in the Naive Parton Model}

In the simplest version of the Parton Model the nucleon is considered to be made of collinear, free constituents, each carrying a fraction $x^{\prime}$ of the nucleon four-momentum. The lepton-nucleon DIS is then described as the incoherent sum of all lepton-constituent quark interactions and the hadronic tensor $W_{\mu \nu}(N)$, Eq. (2.1.8), is given in terms of the elementary quark tensor $w_{\mu \nu}$ by [LEA 85]

$$
\begin{aligned}
W_{\mu \nu}(q ; P, S) & =W_{\mu \nu}^{(S)}(q ; P)+i W_{\mu \nu}^{(A)}(q ; P, S) \\
& =\sum_{q, s} e_{q}^{2} \frac{1}{2 P \cdot q} \int_{0}^{1} \frac{d x^{\prime}}{x^{\prime}} \delta\left(x^{\prime}-x\right) n_{q}\left(x^{\prime}, s ; S\right) w_{\mu \nu}\left(x^{\prime}, q, s\right),
\end{aligned}
$$

where $n_{q}\left(x^{\prime}, s ; S\right)$ is the number density of quarks $q$ with charge $e_{q}$, four-momentum fraction $x^{\prime}$ and spin $s$ inside a nucleon with spin $S$ and four-momentum $P$; the $\Sigma_{q}$ runs over quarks and antiquarks; $x$ is the Bjorken variable (2.1.15) and the quark tensor $w_{\mu \nu}(x, q, s)$ is the same as the leptonic tensor $L_{\mu \nu}$, Eqs. (2.1.2-7), with the replacements $k^{\mu} \rightarrow x P^{\mu}, k^{\prime \mu} \rightarrow x P^{\mu}+q^{\mu}$ and a sum over the unobserved final quark spin $\left(s^{\prime}\right)$ performed. That is:

$$
w_{\mu \nu}(x, q, s)=w_{\mu \nu}^{(S)}(x, q)+i w_{\mu \nu}^{(A)}(x, q, s)
$$

with

$$
\begin{aligned}
w_{\mu \nu}^{(S)}(x, q) & =2\left[2 x^{2} P_{\mu} P_{\nu}+x P_{\mu} q_{\nu}+x q_{\mu} P_{\nu}-x(P \cdot q) g^{\mu \nu}\right] \\
w_{\mu \nu}^{(A)}(x, q, s) & =-2 m_{q} \varepsilon_{\mu \nu \alpha \beta} s^{\alpha} q^{\beta}
\end{aligned}
$$

and the quark mass must for consistency be taken to be $m_{q}=x M$, before and after the interaction with the virtual photon. We will further comment on this point in Section 3.4.

From Eqs. (3.1.1-5) and (3.2.1-4) one obtains the well known Naive Parton Model predictions for the unpolarized nucleon structure functions:

$$
\begin{aligned}
& F_{1}(x)=\frac{1}{2} \sum_{q} e_{q}^{2} q(x) \\
& F_{2}(x)=x \sum_{q} e_{q}^{2} q(x)=2 x F_{1}(x)
\end{aligned}
$$

where the unpolarized quark number densities $q(x)$ are defined as

$$
q(x)=\sum_{s} n_{q}(x, s ; S) .
$$


Similarly, from Eqs. (3.1.6-10) and (3.2.1-4), the polarized nucleon structure functions are obtained:

$$
\begin{aligned}
g_{1}(x) & =\frac{1}{2} \sum_{q} e_{q}^{2} \Delta q(x, S) \\
g_{2}(x) & =0
\end{aligned}
$$

where

$$
\Delta q(x, S)=n_{q}(x, S ; S)-n_{q}(x,-S ; S)
$$

is the difference between the number density of quarks with spin parallel to the nucleon spin $(s=S)$ and those with spin anti-parallel $(s=-S)$. In the Parton Model with free non-interacting quarks of mass $m_{q}=x M$, this quantity cannot depend on the choice of $S$, i.e. $\Delta q(x, S)=\Delta q(x)$; this follows because in such ultra simple case one is allowed to Lorentz transform to the nucleon rest frame, where the quarks too are at rest and where the parton distributions $n_{q}(x, \pm S, S)$, with $S=(0, \hat{\boldsymbol{S}})$, cannot depend on the spin quantization direction $\hat{\boldsymbol{S}}$ [ANS 92].

\subsection{Intrinsic $p_{\perp}$ and $g_{2}(x)$}

The polarized structure function $g_{2}(x)$, Eq. (3.2.9), is zero in the Naive Parton Model where each parton carries a fraction $x$ of the nucleon four-momentum, $p^{\mu}=x P^{\mu}$. However, non zero values of $g_{2}$ can be obtained by allowing the quarks to have an intrinsic Fermi motion inside the nucleon, $p^{\mu}=\left(E_{q}, p_{x}, p_{y}, x^{\prime} P_{z}\right.$ ) (in a nucleon moving along the $\hat{z}$-axis).

Equation (3.2.1) then modifies to [LEA 85]

$$
W_{\mu \nu}(q ; P, S)=\sum_{q, s} e_{q}^{2} \int d^{3} \boldsymbol{p}\left(\frac{P_{0}}{E_{q}}\right) \delta\left(2 p \cdot q-Q^{2}\right) n_{q}(\boldsymbol{p}, s ; S) w_{\mu \nu}(q ; p, s)
$$

which holds in the infinite momentum frame $\left(\left|P_{z}\right| \rightarrow \infty\right)$ and where $n_{q}(\boldsymbol{p}, s ; S)$ is the number density of quarks $q$ with charge $e_{q}$, energy $E_{q}$, three-momentum $\boldsymbol{p}$ and spin $s$ in

a nucleon with spin $S$ and momentum $P$. It is related to the usual quark distribution functions $q(x)$ and $\Delta q(x, s ; S)$, Eqs. $(3.2 .7,10)$, by:

$$
\begin{gathered}
\sum_{s} \int d^{2} \boldsymbol{p}_{\perp} n_{q}(\boldsymbol{p}, s ; S) P_{z}=q\left(x^{\prime}\right) \\
\int d^{2} \boldsymbol{p}_{\perp}\left[n_{q}(\boldsymbol{p}, s ; S)-n_{q}(\boldsymbol{p},-s ; S)\right] P_{z}=\int d^{2} \boldsymbol{p}_{\perp} \Delta q(\boldsymbol{p}, s ; S) P_{z}=\Delta q\left(x^{\prime}, s ; S\right)
\end{gathered}
$$

where we have put $d^{3} \boldsymbol{p}=d^{2} \boldsymbol{p}_{\perp} d x^{\prime} P_{z}$.

Equations (3.1.9) and (3.3.1) yield

$$
g_{2}(x)=\sum_{q, s} e_{q}^{2} \int d^{3} \boldsymbol{p}\left(\frac{P_{0}}{E_{q}}\right) \delta\left(2 p \cdot q-Q^{2}\right) n_{q}(\boldsymbol{p}, s ; S) P_{3}^{\alpha \beta} w_{\alpha \beta}^{(A)}(q ; p, s),
$$

and insertion of the explicit expression of $P_{3}^{\alpha \beta}$, Eqs. $(3.1 .6,8)$ and $w_{\alpha \beta}^{(A)}$, Eq. (3.2.4), yields

$$
\begin{aligned}
g_{2}(x) & =\sum_{q} e_{q}^{2} \frac{4 m_{q}(P \cdot q)^{2}}{b M^{2}(q \cdot S)} \int d^{3} \boldsymbol{p}\left(\frac{P_{0}}{E_{q}}\right) \delta\left(2 p \cdot q-Q^{2}\right) \\
& \times \Delta q(\boldsymbol{p}, s ; S)\left\{(P \cdot q)[(q \cdot S)(s \cdot S)+q \cdot s]-(P \cdot s)\left[(q \cdot S)^{2}+q^{2}\right]\right\}
\end{aligned}
$$


with $b$ as given in Eq. (3.1.8) and the quark mass $m_{q}=\sqrt{E_{q}^{2}-\boldsymbol{p}^{2}}$.

In the nucleon rest frame let $S^{\mu}=(0, \hat{\boldsymbol{S}})$, where $\hat{\boldsymbol{S}}$ is not perpendicular to the $\hat{z}$-axis (along which the nucleon moves in the infinite momentum frame); specify the quark spin by choosing $s^{\mu}=(0, \hat{\boldsymbol{S}})$ in the quark rest frame (which, for $\boldsymbol{p}_{\perp} \neq 0$, is not the nucleon rest frame). Then Eq. (3.3.5), at leading order in $M / P_{z}$, reads [LEA 88]

$$
g_{2}(x)=\frac{1}{2} \sum_{q} e_{q}^{2}\left(1-\frac{x M}{m_{q}}\right) \frac{m_{q}}{x M} \Delta q(x, s ; S)
$$

where we have used Eq. (3.3.3).

As the left hand side cannot depend on $s$ and $S$, Eq. (3.3.6) appears contradictory. However, this is not so since in a fast frame where $p_{\perp}$ is negligible compared to $p_{z}$, both $s^{\mu}$ and $S^{\mu}$, to leading order, are parallel to $P^{\mu}$ so that $\Delta q$ is the helicity distribution and we have

$$
g_{2}(x)=\frac{1}{2} \sum_{q} e_{q}^{2}\left(\frac{m_{q}}{x M}-1\right) \Delta q(x)
$$

where $\Delta q(x)$ is the difference between the number density of quarks with the same helicity as the nucleon and those with opposite helicity. When neglecting the intrinsic $p_{\perp}$ of quarks one has $m_{q}=x M$, so that from Eqs. (3.3.6) and (3.3.7) one recovers the previous result $g_{2}(x)=0$. Thus the introduction of intrinsic $p_{\perp}$ allows a non zero value of $g_{2}(x)$ in the Parton Model, but we see that such value shows an extreme sensitivity to the quark mass, Eq. (3.3.7).

We shall argue, as has been emphasized in [JAF 90], that this throws doubt on all purely Parton Model calculations of $g_{2}$.

\subsubsection{Conflicting Parton Model results for $\boldsymbol{g}_{\mathbf{2}}(\boldsymbol{x})$}

Before discussing the difficulties of the Parton Model with $g_{2}(x)$, and in order to better realize that indeed there are difficulties, we briefly summarize some contradictory statements existing in the literature.

Some authors [IOF 84] claim that

$$
g_{1}(x)+g_{2}(x)=0
$$

whereas Feynman [FEY 72] has

$$
g_{1}(x)+g_{2}(x)=\frac{1}{2} \sum_{q} e_{q}^{2} \Delta q_{T}(x),
$$

where $\Delta q_{T}$ is given by Eq. (3.2.10) with the spin $S$ perpendicular to the nucleon momentum. Moreover, on summing Eqs. (3.2.8) and (3.3.7), one obtains, in terms of helicity densities [LEA 88],

$$
g_{1}(x)+g_{2}(x)=\frac{1}{2} \sum_{q} e_{q}^{2} \frac{m_{q}}{x M} \Delta q(x) .
$$

Finally in [JAC 89] it is argued, using a covariant Parton Model formulation, that

$$
g_{1}(x)+g_{2}(x)=\int_{x}^{1} \frac{d y}{y} g_{1}(y) .
$$

These last authors exploit the experimental data on $g_{1}(x)$ to give predictions for $x g_{2}(x)$ [see Fig. 3.1]. 
In [ANS 92] it is shown that Eq. (3.3.8) is not correct and that Eqs. (3.3.9) and (3.3.10) are compatible when neglecting the intrinsic $p_{\perp}$ of quarks. In section 5.3, we explain why (3.3.11) is incorrect because it neglects twist-3 terms. Nevertheless (3.3.11) may not be a bad approximation, so it should be tested experimentally.

Finally in Fig. 3.2 we show a bag model prediction for $g_{2}(x)$ [JAF 91].

\subsection{Origin of the difficulties with $g_{2}(x)$}

As we have seen the Parton Model does not lead to clearcut results for the spin dependent structure function $g_{2}$. Different authors obtain different results and these are generally incompatible with each other.

Perhaps the most general way to understand this difficulty is to recall that the Parton Model is fundamentally an impulse approximation in which binding effects (i.e. the virtuality) of the struck parton are unimportant for the large transverse momentum reactions under consideration. However, in some cases what one is measuring is not just a cross-section but an asymmetry, i.e. a difference of cross-sections, and it may happen that in this difference the dominant contributions cancel out leaving a result which does depend upon the binding energy or virtuality. Just such effects occur in $g_{2}$. Whenever this happens the result is bound to be unreliable.

We can see this quite directly by reconsidering the antisymmetric part of Eq. (3.3.1), valid in the impulse approximation:

$$
W_{\mu \nu}^{(A)}(q ; P, S)=\sum_{s} e_{q}^{2} \int d^{3} \boldsymbol{p}\left(\frac{P_{0}}{E_{q}}\right) \delta\left(2 p \cdot q-Q^{2}\right) n_{q}(\boldsymbol{p}, s ; S) w_{\mu \nu}^{(A)}(q ; p, s)
$$

where we ignore the sum over the flavours $q$, irrelevant to this issue.

Now consider the calculation of $w_{\mu \nu}^{(A)}$ describing the interaction of the hard photon with the quark, as depicted in Fig. 3.3. The final state quark is a 'free' quark and is on mass-hell: $\left(p^{\prime}\right)^{2}=m_{q}^{2}$. In the impulse approximation also the initial quark is considered to be free and to have the same mass. But to see the danger of this assertion, let us put $p^{2}=m^{2}$, where for the moment we allow $m^{2} \neq m_{q}^{2}$ to represent the fact that the initial quark is really a bound quark. Aside from this we treat the incoming quark as free, i.e. its wave function is taken as the usual free-particle Dirac spinor $u(p, s)$ for a particle of mass $m$.

One then finds

$$
w_{\mu \nu}(q ; p, s)=\frac{1}{2} \operatorname{Tr}\left[\left(1+\gamma_{5} \phi\right)(\not p+m) \gamma_{\mu}\left(\not p+\not q+m_{q}\right) \gamma_{\nu}\right]
$$

from which one obtains

$$
w_{\mu \nu}^{(A)}(q ; p, s)=2 \varepsilon_{\mu \nu \alpha \beta}\left(m_{q} s^{\alpha}\right)\left[\left(1-\frac{m}{m_{q}}\right) p^{\beta}-\frac{m}{m_{q}} q^{\beta}\right] .
$$

Equation (3.4.3) is extremely revealing. We see immediately that for a general $s^{\mu}$ the result is not gauge invariant $\left(q^{\mu} w_{\mu \nu}^{(A)} \neq 0\right)$ unless $m=m_{q}$, in which case we recover the Naive Parton Model, Eq. (3.2.4). Moreover, the offending term, when $m \neq m_{q}$, is not

small in an infinite momentum frame (where the impulse approximation is supposed to be most justifiable) even if $\left(m-m_{q}\right)$ is small.

However, in the special case of longitudinal $(L)$ polarization, if the quark has high momentum so that $m_{q} / p_{z} \ll 1$, the product $\left(m_{q} s_{L}^{\beta}\right) \rightarrow \pm p^{\beta}$ and the gauge non-invariant 
term vanishes because of the antisymmetric $\varepsilon_{\mu \nu \alpha \beta}$. For the calculation of $g_{1}(x)$ it is the longitudinal polarization that is relevant and there is no unreasonable sensitivity to whether or not $m$ equals $m_{q}$. Moreover, since the limit $\lim _{m_{q} \rightarrow 0}\left(m_{q} S_{L}^{\beta}\right)$ is finite there is also no crucial sensitivity to whether one works with massive or massless quarks. Thus $g_{1}(x)$ can be calculated unambigously in the Parton Model.

Quite the opposite happens for $g_{2}(x)$ where the transverse spin is relevant. There is an extreme sensitivity to whether or not $m$ equals $m_{q}$ and one cannot expect to make a reliable calculation of $g_{2}(x)$ in the Parton Model.

One can put the case even more forcefully. The whole point of quarks is that, in their pointlike interaction with a hard photon, they produce large momentum transfer reactions which we are trying to generate for the photon-hadron interactions. But even if we define the model by insisting that $m=m_{q}$, comparing the expression (3.4.3) with the general structure of $W_{\mu \nu}^{(A)}$ for a spin $1 / 2$ particle (2.1.19), we see that

$$
\left.g_{2}(x)\right|_{\text {quark }}=0 .
$$

Thus the hard photon-free quark interaction does not possess the cross-section asymmetry which we are seeking to explain in the photon-hadron interaction. It is clearly unrealistic therefore to try to produce such an asymmetry from quark-partons.

\subsection{Weak interaction structure functions in the Naive Parton Model}

We have seen in Section 2.2 that, when taking into account weak interactions, the most general form of the hadronic tensor, Eq. (2.2.7), contains 8 independent structure functions, 3 unpolarized and 5 polarized ones. On the other hand, in the Naive Parton Model, the hadronic tensor is given by (see Eq. (3.2.1))

$$
W_{\mu \nu}^{i}(q ; P, S)=\sum_{q} \frac{1}{2(P \cdot q) x}\left[n_{q}(x, S ; S) w_{\mu \nu}^{i, q}(x, q, S)+n_{q}(x,-S ; S) w_{\mu \nu}^{i, q}(x, q,-S)\right]
$$

where we have explicitely performed the sum over $s= \pm S$. The index $i$ denotes the different kinds of contributions $(i=\gamma, \gamma Z, Z, W)$ and the $\sum_{q}$ runs over quarks and antiquarks. The $w_{\mu \nu}^{i, q}$ are the (flavour dependent) quark tensors, given by:

$$
\begin{aligned}
w_{\mu \nu}^{\gamma, q}(x, q, s) & =\sum_{s^{\prime}} e_{q}^{2}\left[\bar{u}\left(p^{\prime}, s^{\prime}\right) \gamma_{\mu} u(p, s)\right]^{*}\left[\bar{u}\left(p^{\prime}, s^{\prime}\right) \gamma_{\nu} u(p, s)\right] \\
w_{\mu \nu}^{\gamma Z, q}(x, q, s) & =\sum_{s^{\prime}} e_{q}\left[\bar{u}\left(p^{\prime}, s^{\prime}\right) \gamma_{\mu}\left(g_{V}-g_{A} \gamma_{5}\right)_{q} u(p, s)\right]^{*}\left[\bar{u}\left(p^{\prime}, s^{\prime}\right) \gamma_{\nu} u(p, s)\right] \\
& +\sum_{s^{\prime}} e_{q}\left[\bar{u}\left(p^{\prime}, s^{\prime}\right) \gamma_{\mu} u(p, s)\right]^{*}\left[\bar{u}\left(p^{\prime}, s^{\prime}\right) \gamma_{\nu}\left(g_{V}-g_{A} \gamma_{5}\right) u(p, s)\right] \\
w_{\mu \nu}^{Z, q}(x, q, s) & =\sum_{s^{\prime}}\left[\bar{u}\left(p^{\prime}, s^{\prime}\right) \gamma_{\mu}\left(g_{V}-g_{A} \gamma_{5}\right)_{q} u(p, s)\right]^{*}\left[\bar{u}\left(p^{\prime}, s^{\prime}\right) \gamma_{\nu}\left(g_{V}-g_{A} \gamma_{5}\right)_{q} u(p, s)\right] \\
w_{\mu \nu}^{W, q}(x, q, s) & =\sum_{s^{\prime}, q^{\prime}}\left[\bar{u}\left(p^{\prime}, s^{\prime}\right) \gamma_{\mu}\left(1-\gamma_{5}\right) u(p, s)\right]^{*}\left[\bar{u}\left(p^{\prime}, s^{\prime}\right) \gamma_{\nu}\left(1-\gamma_{5}\right) u(p, s)\right]\left|(V)_{q q^{\prime}}\right|^{2}
\end{aligned}
$$

where $p=x P, p^{\prime}=p+q, s$ and $s^{\prime}$ are respectively the momentum and spin four-vectors of the initial and final quarks. $\left(g_{V}\right)_{q}$ and $\left(g_{A}\right)_{q}$ are the vector and axial couplings of the quark of flavour $q$ to the $Z_{0}$ :

$$
\begin{aligned}
\left(g_{V}\right)_{u, c}=\frac{1}{2}-\frac{4}{3} \sin ^{2} \theta_{W} & \left(g_{A}\right)_{u, c}=\frac{1}{2} \\
\left(g_{V}\right)_{d, s}=-\frac{1}{2}+\frac{2}{3} \sin ^{2} \theta_{W} & \left(g_{A}\right)_{d, s}=-\frac{1}{2} .
\end{aligned}
$$


In case of charged current, negatively charged leptons couple to $u$-type quarks (or $\bar{d}$-type antiquarks) and positively charged leptons couple to $d$-type quarks (or $\bar{u}$-type antiquarks); one has also to take into account the proper Cabibbo-Kobayashi-Maskawa matrix elements $(V)_{q q^{\prime}}$ occuring in the transition coupling from a flavour $q$ to a flavour $q^{\prime}$. However, if we consider the contribution of four flavours $(u, d, s$ and $c)$, one always has $\sum_{q^{\prime}}\left|(V)_{q q^{\prime}}\right|^{2}=\cos ^{2} \theta_{c}+\sin ^{2} \theta_{c}=1$, where $\theta_{c}$ is the Cabibbo angle. Equations (3.5.2) and (3.5.3) hold for quarks; for antiquarks one should only replace $\gamma_{5}$ with $-\gamma_{5}$ in Eqs. (3.5.2) leaving all other expressions unchanged.

Explicit expressions for Eqs. (3.5.2) can be obtained from the general form

$$
\begin{aligned}
w_{\mu \nu}(x, q, s) & =\sum_{s^{\prime}}\left[\bar{u}\left(p^{\prime}, s^{\prime}\right) \gamma_{\mu}\left(v_{1}-a_{1} \gamma_{5}\right) u(p, s)\right]^{*}\left[\bar{u}\left(p^{\prime}, s^{\prime}\right) \gamma_{\nu}\left(v_{2}-a_{2} \gamma_{5}\right) u(p, s)\right] \\
& =2\left(a_{1} a_{2}+v_{1} v_{2}\right)\left[2 p_{\mu} p_{\nu}-p \cdot q g_{\mu \nu}\right]-4 a_{1} a_{2} m_{q}^{2} g_{\mu \nu} \\
& -2 v_{1} a_{2} m_{q}\left[2 p_{\mu} s_{\nu}-s \cdot q g_{\mu \nu}\right]-2 a_{1} v_{2} m_{q}\left[2 s_{\mu} p_{\nu}-s \cdot q g_{\mu \nu}\right] \\
& +2 i \varepsilon_{\mu \nu \alpha \beta}\left[\left(v_{1} a_{2}+a_{1} v_{2}\right) p^{\alpha} q^{\beta}+2 a_{1} a_{2} m_{q} p^{\alpha} s^{\beta}+\left(a_{1} a_{2}+v_{1} v_{2}\right) m_{q} q^{\alpha} s^{\beta}\right]
\end{aligned}
$$

by properly fixing the values of $v_{1,2}$ and $a_{1,2}$; in Eq. (3.5.4) we have dropped, as usual, terms proportional to $q^{\mu}$ or $q^{\nu}$ which, when contracted with the leptonic tensor $L^{\mu \nu}$ give negligible contributions proportional to $m / E$.

Equations (3.5.1-4) give the Parton Model prediction of the hadronic tensor $W_{\mu \nu}^{i}$; by comparing it with the general expression (2.2.7) one obtains the naive quark-parton model results for the nucleon structure functions. For completeness we list all of them here, starting from the electromagnetic case $(i=\gamma)$, for which we recover Eqs. $(3.2 .5,6)$ :

$$
\begin{aligned}
F_{1}^{\gamma}=\frac{1}{2} \sum_{f} e_{q_{f}}^{2}\left(q_{f}+\bar{q}_{f}\right) & F_{2}^{\gamma}=2 x F_{1}^{\gamma} \\
g_{1}^{\gamma}=\frac{1}{2} \sum_{f} e_{q_{f}}^{2}\left(\Delta q_{f}+\Delta \bar{q}_{f}\right) & g_{2}^{\gamma}=0
\end{aligned}
$$

where $q_{f}=u, d, s, c ; u$ stands for the number density of quarks $u$ and so on. The interference contribution $(i=\gamma Z)$ is:

$$
\begin{aligned}
F_{1}^{\gamma Z} & =\sum_{f} e_{q_{f}}\left(g_{V}\right)_{q_{f}}\left(q_{f}+\bar{q}_{f}\right) \quad F_{2}^{\gamma Z}=2 x F_{1}^{\gamma Z} \\
F_{3}^{\gamma Z} & =2 \sum_{f} e_{q_{f}}\left(g_{A}\right)_{q_{f}}\left(q_{f}-\bar{q}_{f}\right) \\
g_{1}^{\gamma Z} & =\sum_{f} e_{q_{f}}\left(g_{V}\right)_{q_{f}}\left(\Delta q_{f}+\Delta \bar{q}_{f}\right) \\
g_{2}^{\gamma Z} & =g_{4}^{\gamma Z}=0 \\
g_{3}^{\gamma Z} & =2 x \sum_{f} e_{q_{f}}\left(g_{A}\right)_{q_{f}}\left(\Delta q_{f}-\Delta \bar{q}_{f}\right)=2 x g_{5}^{\gamma Z}
\end{aligned}
$$

and the purely weak interaction $(i=Z)$ leads to:

$$
\begin{aligned}
F_{1}^{Z} & =\frac{1}{2} \sum_{f}\left(g_{V}^{2}+g_{A}^{2}\right)_{q_{f}}\left(q_{f}+\bar{q}_{f}\right) \quad F_{2}^{Z}=2 x F_{1}^{Z} \\
F_{3}^{Z} & =2 \sum_{f}\left(g_{V} g_{A}\right)_{q_{f}}\left(q_{f}-\bar{q}_{f}\right) \\
g_{1}^{Z} & =\frac{1}{2} \sum_{f}\left(g_{V}^{2}+g_{A}^{2}\right)_{q_{f}}\left(\Delta q_{f}+\Delta \bar{q}_{f}\right)
\end{aligned}
$$




$$
\begin{aligned}
g_{2}^{Z} & =-\frac{1}{2} \sum_{f}\left(g_{A}^{2}\right)_{q_{f}}\left(\Delta q_{f}+\Delta \bar{q}_{f}\right) \\
g_{3}^{Z} & =2 x \sum_{f}\left(g_{V} g_{A}\right)_{q_{f}}\left(\Delta q_{f}-\Delta \bar{q}_{f}\right)=2 x g_{5}^{Z} \\
g_{4}^{Z} & =0 .
\end{aligned}
$$

In case of charged current $(i=W)$, on performing explicitely the $\sum_{f}$, one obtains, for $\ell^{-} N \rightarrow \nu X$ processes:

$$
\begin{aligned}
F_{1}^{W^{-}} & =u+c+\bar{d}+\bar{s} \quad F_{2}^{W^{-}}=2 x F_{1}^{W^{-}} \\
F_{3}^{W^{-}} & =2(u+c-\bar{d}-\bar{s}) \\
g_{1}^{W^{-}} & =(\Delta u+\Delta c+\Delta \bar{d}+\Delta \bar{s})=-2 g_{2}^{W^{-}} \\
g_{3}^{W^{-}} & =2 x(\Delta u+\Delta c-\Delta \bar{d}-\Delta \bar{s})=2 x g_{5}^{W^{-}} \\
g_{4}^{W^{-}} & =0 .
\end{aligned}
$$

$\ell^{+} N \rightarrow \bar{\nu} X$ processes probe different quark flavours and one obtains the corresponding expressions of the structure functions $F_{j}^{W^{+}}$and $g_{j}^{W^{+}}$by the flavour interchanges $d \leftrightarrow u$ and $s \leftrightarrow c$ in the above Eqs. (3.5.8).

Notice that in the naive quark-parton model the structure functions $g_{4}^{i}$ are always zero and one finds $g_{3}^{i}=2 x g_{5}^{i}$ for any $i=\gamma, \gamma Z, Z, W$. The functions $g_{2}^{i}$ are nonzero only for pure weak interactions (both in neutral and charged current processes). It is interesting to note that, in case of neutral current, the integral of $g_{2}^{Z}$ is directly proportional to the total spin carried by the quarks and antiquarks, as can be seen from Eqs. (3.5.7) and (3.5.3). Particular combinations of the structure functions can single out interesting quark and quark spin information; some examples are discussed in [ANS 93].

\section{Phenomenological analysis of the data on $\boldsymbol{g}_{\mathbf{1}}(\boldsymbol{x})$ and its first moment $\Gamma_{1}$}

As explained in Section 2.1.3 the measurement of the cross-section asymmetry using a longitudinally polarized lepton beam on a longitudinally polarized hadron target may be interpreted as essentially a measurement of the spin dependent structure function $g_{1}\left(x, Q^{2}\right)$. Experimental data on $g_{1}^{p}$ were first obtained at SLAC [ALG 78] as early as 1978 using an electron beam. Further information came from the SLAC-Yale group [BAU 83] in 1983, with fascinating implications about the internal structure of the proton. And then, more recently, very startling results were obtained by the European Muon Collaboration (EMC) who scattered a longitudinally polarized muon beam of energy 100-200 GeV on a longitudinally polarized hydrogen target at CERN [ASH 88, 89]. The unexpectedly low asymmetry found by the EMC led to what was termed a "spin crisis in the Parton Model" [LEA 88] and raised serious questions as to how the spin of the proton is built up from the spins of its constituents. It also seemed, for a while, to imply the first failure of the Parton Model D.

The EMC result catalysed a great deal of theoretical research and the "crisis" is now believed to be resolved as a consequence of the discovery of a deep and beautiful

1) It is often forgotten that there are in a sense other failures of the NPM, namely the experimental observations of large transverse polarization in inclusive $\Lambda$-production at high $p_{T}$ first observed in 1976 and of left-right asymmetry in high $p_{T}$ inclusive pion production on a transversely polarized target (see e.g. [HEL 91]). 
connection between the observable measured by the EMC and the axial anomaly [EFR 88]. Nonetheless the situation is not yet fully settled and there are subtle non-perturbative issues and ambiguities in limiting procedures which will be discussed in Section 6 .

Two new experiments are under way to check the EMC measurement of $g_{1}^{p}$ and to measure the analogous neutron function $g_{1}^{n}$. First results have now been presented [ADE 93, 94; ANT 93] and we shall discuss their implications in Section 4.5. Our discussion in this Section will largely rely on the EMC experiment, since this is the experiment at largest $\left\langle Q^{2}\right\rangle$ and should thus be least sensitive to higher twist contributions. It is important to realize that even if the EMC results turn out to be incorrect, the theoretical discoveries catalysed by them remain valid and interesting. In fact at the time of going to press, the SMC has just issued results at the same $\left\langle Q^{2}\right\rangle$ as the EMC experiment [ADA 94] and they are in substantial agreement with those of EMC. We will comment briefly on the new measurement in Section 4.2.2.

We shall begin by analysing the data in the simple Parton Model. We shall find ourselves in difficulty and will therefore invoke the more sophisticated operator product expansion with its QCD corrections. This will be seen to offer no significant help and we will be forced to conclude that the standard approach is in trouble. A possible resolution of these difficulties in terms of an anomalous gluonic contribution will be discussed in Section 7, where we deal with the first moment of $g_{1}^{p}(x)$ and in Section 8.4 where we consider the $x$-dependence.

\subsection{The SLAC-Yale and EMC data: quark distributions near $x=1$}

As derived in Section 3.2, in the Naive Parton Model one has

$$
g_{1}^{p}(x)=\frac{1}{2}\left\{\frac{4}{9}[\Delta u(x)+\Delta \bar{u}(x)]+\frac{1}{9}[\Delta d(x)+\Delta \bar{d}(x)]+\frac{1}{9}[\Delta s(x)+\Delta \bar{s}(x)]\right\}
$$

where we assume that the contribution of heavy quarks is negligible.

Before discussing the data on $g_{1}^{p}(x)$ itself it will be interesting to look at the virtual Compton scattering asymmetry $A_{1}(x)$ introduced in Appendix A. Taking the approximate form

$$
A_{1}(x) \approx \frac{g_{1}(x)}{F_{1}(x)}
$$

and using the standard result

$$
F_{1}(x)=\frac{1}{2}\left\{\frac{4}{9}[u(x)+\bar{u}(x)]+\frac{1}{9}[d(x)+\bar{d}(x)]+\frac{1}{9}[s(x)+\bar{s}(x)]\right\}
$$

one has, using (4.1.1),

$$
A_{1}(x) \approx \frac{4[\Delta u(x)+\Delta \bar{u}(x)]+\Delta d(x)+\Delta \bar{d}(x)+\Delta s(x)+\Delta \bar{s}(x)}{4[u(x)+\bar{u}(x)]+d(x)+\bar{d}(x)+s(x)+\bar{s}(x)} .
$$

The EMC and early SLAC-Yale data [ALG 78; BAU 83] on $A_{1}(x)$ are shown in Fig. 4.1. The errors are, of course, large, but there is a discernible trend towards the maximum possible value $A_{1}=1$ as $x \rightarrow 1$.

Given that we know empirically that $u(x) \gg d(x), s(x), \bar{q}(x)$ as $x \rightarrow 1$ and that one must have $|\Delta q(x)| \leq q(x)$ for each quark flavour, the behaviour $A_{1} \rightarrow 1$ as $x \rightarrow 1$ suggests that

$$
\left.\Delta u(x)\right|_{x \rightarrow 1} \rightarrow u(x) .
$$

This implies that those $u$ quarks which carry a large fraction of the longitudinally polarized proton momentum are highly polarized along the direction of the proton spin. 


\subsection{Analysis of data in the framework of the Operator Product Expansion}

As will be discussed in Section 5, the operator product expansion gives results for the moments of $g_{1,2}$ in terms of hadronic matrix elements of certain operators multiplied by perturbatively calculable coefficient functions. The general result for the moments of $g_{1}$ is given in (5.2.1). If, however, the fields involved in the electromagnetic currents in (5.1.1) are treated as free quark fields the results simplify via (5.1.10) - in effect the current commutator in (5.1.1) can be explicitely evalutated - and one obtains

$$
\Gamma_{1}^{p}=\int_{0}^{1} d x g_{1}^{p}(x)=\frac{1}{12}\left\{a_{3}+\frac{1}{\sqrt{3}} a_{8}+\frac{4}{3} a_{0}\right\}
$$

where the $a_{i}$ are [aside from a factor specified below in (4.2.7 and 8)] hadronic matrix elements of the octet of quark $S U(3)_{F}$ axial-vector currents $J_{5 \mu}^{j}(j=1, \ldots, 8)$ and the flavour singlet axial current $J_{5 \mu}^{0}$ taken between proton states of definite momentum and spin direction. (In practice we usually use helicity states). The precise definitions are given below.

In the following we shall explain how the values of $a_{3}$ and $a_{8}$ can be obtained from data on hyperon $\beta$-decay. The EMC measurement of $\Gamma_{1}^{p}$ can thus, via (4.2.1) be construed as the first ever measurement of the singlet matrix element $a_{0}$ and it will turn out that the value of $a_{0}$ is unexpectedly small.

We shall show that in the Naive Parton Model

$$
a_{0}=2 S_{z}^{q}
$$

where $S_{z}^{q}$ is the component of the total quark spin (carried by all quarks and antiquarks) in the direction of motion of a proton of helicity $+1 / 2$. That $S_{z}^{q}$ turns out to be almost compatible with zero is a great surprise. Naively it might have been expected to be fairly close to $+1 / 2$ implying $a_{0} \simeq 1$. When the quark fields are treated as interacting fields there are small perturbative corrections to (4.2.1) (see Section 4.4.1) but these have no significant effect on the argument below.

The octet currents are

$$
J_{5 \mu}^{j}=\overline{\boldsymbol{\psi}} \gamma_{\mu} \gamma_{5}\left(\frac{\boldsymbol{\lambda}_{j}}{2}\right) \boldsymbol{\psi} \quad(j=1,2, \ldots, 8)
$$

where the $\boldsymbol{\lambda}_{j}$ are the usual Gell-Mann matrices and $\boldsymbol{\psi}$ is a column vector in flavour space

$$
\boldsymbol{\psi}=\left(\begin{array}{c}
\psi_{u} \\
\psi_{d} \\
\psi_{s}
\end{array}\right)
$$

and the flavour singlet current is

$$
J_{5 \mu}^{0}=\overline{\boldsymbol{\psi}} \gamma_{\mu} \gamma_{5} \boldsymbol{\psi}
$$

(Note that $J_{5 \mu}^{0}$ is sometimes defined like (4.2.3) with a flavour matrix $\boldsymbol{\lambda}_{0} / 2=(1 / \sqrt{6}) \boldsymbol{I}$.) The question of the conservation of the non-singlet axial currents will be discussed in Section 6.1.

The proton states will be labelled by the four-momentum $P^{\mu}$ and the covariant spin vector $S^{\mu}(\lambda)$ corresponding to definite helicity $\lambda$. Recall that

$$
S \cdot P=0 \quad S^{2}=-1 .
$$


The forward matrix elements of the $J_{5 \mu}^{j}$ can only be proportional to $S^{\mu}$ and the $a_{j}$ are conventionally defined by

$$
\begin{aligned}
\left\langle P, S\left|J_{5 \mu}^{j}\right| P, S\right\rangle & =M a_{j} S_{\mu} \\
\left\langle P, S\left|J_{5 \mu}^{0}\right| P, S\right\rangle & =2 M a_{0} S_{\mu} .
\end{aligned}
$$

The relative factor of 2 in (4.2.7 and 8) reflects the fact that the $S U(3)$ currents are defined using the generators of the group i.e. $\boldsymbol{\lambda}_{j} / 2$ in (4.2.3).

Analogous to (4.2.3) one introduces an octet of vector currents

$$
J_{\mu}^{j}=\overline{\boldsymbol{\psi}} \gamma_{\mu}\left(\frac{\boldsymbol{\lambda}_{j}}{2}\right) \boldsymbol{\psi} \quad(j=1, \ldots, 8)
$$

which are conserved currents to the extent that $S U(3)_{F}$ is a symmetry of the strong interactions.

\subsubsection{Information from hyperon $\boldsymbol{\beta}$-decay}

Consider now the $\beta$-decays of the spin $1 / 2$ hyperons. If we use the standard $S U(3)$ labelling [BAI 82] $B_{j}$ for the hyperons then the hadronic transition involved is controlled by matrix elements of the form $\left\langle B_{i}\left|h_{+}^{\mu}\right| B_{k}\right\rangle$ where $h_{+}^{\mu}$ is the charged hadronic current that couples to the $W$ boson in the electroweak Lagrangian. It is typically of the form of a Kobayashi-Maskawa matrix element multiplied by some combination of the currents $J_{\mu}^{j}$ and $J_{5 \mu}^{j}$. For example $\Delta Q=\Delta S=1$ transitions $\left(\Lambda \rightarrow p ; \bar{\Sigma} \rightarrow n ; \Xi^{-} \rightarrow \Lambda\right)$ are controlled by the current

$$
V_{u s} \bar{u} \gamma^{\mu}\left(1-\gamma_{5}\right) s=V_{u s}\left[J_{\mu}^{4}+i J_{\mu}^{5}-J_{5 \mu}^{4}-i J_{5 \mu}^{5}\right]
$$

If we now assume:

a) that the 8 spin $1 / 2$ hyperons form an octet under $S U(3)_{F}$;

b) that the currents $J_{\mu}^{j}, J_{5 \mu}^{j}(j=1, \ldots, 8)$ transform as an octet under $S U(3)_{F}$ with $J_{\mu}^{j}, J_{5 \mu}^{j}$ conserved, (the conservation of the axial current will be discussed in Section $6.1)$;

c) that the momentum transfer and mass differences in the hadronic transitions are negligible;

then all the hyperon $\beta$-decays are described in terms of two constants, $F$ and $D$ which occur in the matrix elements of the octet of axial currents [BAI 82]:

$$
\left\langle B_{j} ; P, S\left|J_{5 \mu}^{i}\right| B_{k} ; P, S\right\rangle=2 M_{B} S_{\mu}\left\{-i f_{i j k} F+d_{i j k} D\right\} \quad(i, j, k=1, \ldots, 8)
$$

where the $f_{i j k}$ and $d_{i j k}$ are the usual $S U(3)_{F}$ group constants. The structure of (4.2.11) is dictated by the $S U(3)$ transformation properties of the LHS and is an example of the Wigner-Eckart theorem. It should be noted that $J_{5 \mu}^{0}$ does not play any role in the weak interactions.

An analysis in 1983 of hyperon decay data [WA2 83] seemed to be in good agreement with the above and led to the values

$$
F=0.477 \pm 0.012, \quad D=0.756 \pm 0.011 .
$$

If now we use the standard $S U(3)_{F}$ assignments for the baryon octect [BAI 82] we find from (4.2.11) that

$$
\begin{array}{r}
a_{3}=F+D \\
a_{8}=\frac{1}{\sqrt{3}}(3 F-D) .
\end{array}
$$


We see, therefore, that the study of hyperon $\beta$-decay provides us, via (4.2.12), with the values of $a_{3}$ and $a_{8}$, two of the three parameters occuring in (4.2.1). However there is some doubt as to the reliability of the values quoted in (4.2.12), and for that reason we shall adopt a slightly different strategy. Ultimately, however, it will be seen that the essential result is not sensitive to this issue.

It is straightforward to demonstrate, using only isotopic spin invariance, that

$$
a_{3}=g_{A},
$$

where $g_{A}$ is the axial coupling $\left(=G_{A} / G_{V}\right.$ in the language of the old Cabibbo theory) which governs neutron $\beta$-decay. We prefer therefore to use the very accurate measurement of $g_{A}$ [PDG 92]

$$
a_{3}=g_{A}=1.2573 \pm 0.0028
$$

rather than the value $F+D=1.233 \pm 0.016$ that follows from (4.2.12).

\subsubsection{The EMC data on $\Gamma_{1}^{p}$ and its consequences}

Our main interest will be in the matrix element of the flavour singlet axial current, i.e. in $a_{0}$. From (4.2.1) we have

$$
a_{0}=\frac{3}{4}\left\{12 \Gamma_{1}^{p}-a_{3}-\frac{1}{\sqrt{3}} a_{8}\right\} .
$$

The EMC data on $x g_{1}^{p}(x)$ are shown in Fig. 4.2. Of particular interest, and indeed the source of all the recent theoretical research in this subject, is the first moment

$$
\Gamma_{1}^{p} \equiv \int_{0}^{1} d x g_{1}^{p}(x)
$$

Since experimental measurements cannot reach $x=0$ or 1 an extrapolation of the data is always required in evaluating (4.2.18). The region $x \rightarrow 1$ is harmless but the extrapolation to $x=0$ is potentially tricky. The dashed curve in Fig. 4.2 shows the form of the extrapolation used by the EMC for the value of $\int_{x}^{1} d x^{\prime} g_{1}^{p}\left(x^{\prime}\right)$ as function of $x$ for $x \rightarrow 0$. It is seen that the extrapolation looks perfectly reasonable (but beware the logorithmic scale) and it led to the value $\Gamma_{1}^{p}=0.126 \pm 0.010 \pm 0.015$.

Recall [see (2.1.31)] that to extract $g_{1}(x)$ from the measurement of the asymmetry $A_{\|}$one has to utilize information on $F_{2}\left(x, Q^{2}\right)$ and $R\left(x, Q^{2}\right)$ which are studied in unpolarized DIS. Since the EMC experiment was carried out there have been improved determinations of $F_{2}$ by the NMC group [AMA 92] and of $R$ at SLAC [WHI 90]. As a consequence there is a small change and one has [ELL 93]

$$
\Gamma_{1}^{p}\left[\left\langle Q^{2}\right\rangle=10.7\right]=0.128 \pm 0.013 \pm 0.019
$$

where we have indicated the mean value of $Q^{2}$ for the experiment.

In Fig. 4.3 we show the new SMC data [ADA 94] on $g_{1}^{p}(x)$ at $\left\langle Q^{2}\right\rangle=10(\mathrm{GeV} / \mathrm{c})^{2}$ compared with the EMC data. There is excellent agreement in general where the measurements overlap. The new data, which extend to smaller value of $x$ give some indication that $g_{1}^{p}(x)$ may be increasing as $x \rightarrow 0$, though this trend may not be significant. The SMC value for the first moment is $\Gamma_{1}^{p}\left[\left\langle Q^{2}\right\rangle=10\right]=0.136 \pm 0.011 \pm 0.011$, perfectly consistent with the EMC result (4.2.19). 
This result is considerably smaller than the value $0.188 \pm 0.004$ expected on the basis of the Ellis-Jaffe sum rule which will be discussed in Section 4.3. Several attempts were made to try to explain this discrepancy, initially focusing on the question as to whether (4.2.19) can be considered as a reliable number to compare with the theory. If we substitute the value of $\Gamma_{1}^{p}(4.2 .19)$ into (4.2.17) we obtain

$$
a_{0}=\frac{3}{4}\left\{(0.279 \pm 0.156 \pm 0.228)-\frac{1}{3}(3 F-D)\right\} \text {. }
$$

The values $(4.2 .12)$ yield $(3 F-D) / 3=0.225 \pm 0.013$ which implies $a_{0}=0.040 \pm 0.117 \pm$ 0.171. However a more recent analysis of hyperon decays obtains [HSU 88]

$$
F=0.46 \pm 0.01 \quad D=0.79 \pm 0.01 \quad \frac{1}{3}(3 F-D)=0.20 \pm 0.01
$$

which implies

$$
a_{0}=0.06 \pm 0.12 \pm 0.17 .
$$

As mentioned earlier one might have expected $a_{0} \simeq 1$ so that the value in $(4.2 .22)$ is surprisingly small.

This unintuitive result and the disagreement with the Ellis-Jaffe sum rule inspired several attempts to question the reliability of the EMC value for $\Gamma_{1}^{p}$. Because of its fundamental importance we turn to this question. We believe that only two issues require serious consideration: the question of the extrapolation to $x=0$ and the question of higher twist effects.

\subsubsection{The EMC data: extrapolation to $\boldsymbol{x}=\mathbf{0}$}

The extrapolation to small $x$ was queried by Close and Roberts [CLO 88] who stressed that the extraction of $g_{1}(x)$ from the data, at small $x$, is dependent upon the assumed behavior as $x \rightarrow 0$ of both $g_{1}(x)$ and the usual structure function $F_{2}(x)$, and that, in particular, the latter may have a more singular behavior than is given by the usual Regge analysis of small $x$ behavior.

We are convinced that the Regge behavior

$$
g_{1}(x) \sim x^{-\alpha_{a_{1}}}
$$

where $\alpha_{a_{1}}(t)$ is the Regge trajectory of the $a_{1}$ meson (previously referred to as the $A_{1}$ ), $\alpha \equiv \alpha(0) \simeq-0.14 \pm 0.20$, is correct, and that it is not possible to have a contribution to $g_{1}(x)$ from the $P \otimes P$ cut [HEI 73]. The reason is that only Regge poles or cuts with $G(-1)^{T} \sigma=-1$ can contribute to those virtual Compton scattering amplitudes that are relevant to $g_{1}(x)$. (Here $T$ is the $t$-channel isospin, $G$ is the $G$-parity and $\sigma$ the signature). However, it is possible to have a three-pomeron cut, but its contribution relative to $a_{1^{-}}$ exchange is suppressed by both a factor $(m / Q)(\ln \nu)^{-5}$ and a small numerical coefficient. Also we are reluctant to accept that the non-Regge singular behavior of $F_{2}(x)$ can be relevant at the values of $Q^{2}$ involved in the EMC experiment. It seems generally accepted that the extrapolation to small $x$ used by the EMC is reliable and is not the cause of their peculiar result.

It is interesting that there is some support for the validity of the EMC extrapolation which comes from a totally different source, namely elastic $\nu p \rightarrow \nu p$. In the latter process the momentum transfer dependence of the differential cross-section gives information on 
the axial form factor $G_{A}\left(Q^{2}\right)$ [AHR 87] whose value at $Q^{2}=0$ is directly related to the $a_{j}$ appearing in DIS. One has

$$
G_{A}(0)=\frac{1}{2}\left\{a_{3}+\frac{1}{\sqrt{3}} a_{8}-\frac{1}{3} a_{0}\right\} .
$$

It is not absolutely straightforward to obtain $G_{A}(0)$ from the data, since it is somewhat dependent on the form of the fit assumed for the $Q^{2}$ variation in the measured range $0.45 \leq Q^{2} \leq 1.05(\mathrm{GeV} / \mathrm{c})^{2}$. Thus, although no extrapolation in $x$ is required, one does have to make an assumption of smooth behaviour in $Q^{2}$ as $Q^{2} \rightarrow 0$. The value of $G_{A}(0)$ given in [AHR 87] corresponds to

$$
a_{0}-\sqrt{3} a_{8}=-0.45 \pm 0.27 .
$$

Using (4.2.14) and (4.2.21) yields

$$
a_{0}=0.14 \pm 0.27
$$

which is perfectly consistent with the EMC value in (4.2.22).

The reader is warned that the $\nu p \rightarrow \nu p$ data are sometimes used as an argument in favour of a large polarized strange quark contribution $\Delta s$. This interpretation is only valid in the Naive Parton Model where $\frac{1}{3}\left[a_{0}-\sqrt{3} a_{8}\right]=\Delta s$. As will be discussed in Section 8.5 there is also a gluonic contribution arising from the axial anomaly.

\subsubsection{The EMC data : higher twist effects}

The possibility that significant higher twist effects at the values of $Q^{2}$ in the EMC experiment might invalidate a comparison of the data with the Parton Model or with the leading twist operator product expansion, was studied by Anselmino, Ioffe and Leader [ANS 89] in an approach based upon compatibility with the Gerasimov, Drell, Hearn sum rule. A more consistent treatment was later developed by Burkert and Ioffe [BUR 92, 93].

Higher twist effects have also been approached in a quite different way by Balitsky, Braun and Kolesnichenko [BAL 90, 93] based upon the study of higher twist operators and QCD sum rules. The latter authors find much smaller corrections than in [ANS 89] and in [BUR 92, 93], but the uncertainties in their approach are intrinsically difficult to assess. Also there is some question as to the correctness of the whole approach [IOF 92].

We shall proceed to explain the two methods. Ultimately we feel that higher twist effects are unimportant in the EMC experiment but are surely not negligible in the new experiments at $Q^{2}=2$ and $4.6(\mathrm{GeV} / \mathrm{c})^{2}$.

a) The Gerasimov, Drell, Hearn sum rule approach.

The key point is that $\Gamma_{1}^{p}$ is connected to the Gerasimov-Drell-Hearn (GDH) sum rule [GER 66, DRE 66] for forward scattering of real photons on nucleons and that this indicates rapid $Q^{2}$-dependences which might still have consequences at the EMC $Q^{2}$ values.

Define

$$
I_{p}\left(Q^{2}\right) \equiv \frac{2 M^{2}}{Q^{2}} \Gamma_{1}^{p}\left(Q^{2}\right)
$$

Then at large $Q^{2}$

$$
I_{p}\left(Q^{2}\right) \rightarrow \frac{2 M^{2}}{Q^{2}} \Gamma_{1}^{p}\left(Q^{2}\right)_{A s}
$$


where $\Gamma_{1}^{p}\left(Q^{2}\right)_{A s}$ is the asymptotic form given by say the lowest twist contribution to the operator product expansion and which is, empirically, positive. But, according to GDH,

$$
I_{p}(0)=-\frac{\kappa_{p}^{2}}{4}
$$

where $\kappa_{p}$ is the anomalous magnetic moment of the proton $\left(\kappa_{p}=1.79\right)$. Figure 4.4 shows $I_{p}\left(Q^{2}\right)$ as given by DIS data and the value at $Q^{2}=0$. (Also shown is $I_{p}\left(Q^{2}\right)-I_{n}\left(Q^{2}\right)$ relevant to the Bjorken sum rule). Since the $Q^{2}$-dependence of $\Gamma_{1}^{p}\left(Q^{2}\right)_{A s}$, as given by perturbative QCD, is slow and logorithmic, the behaviour of $I\left(Q^{2}\right)$ implies strong higher twist effects in $\Gamma_{1}^{p}\left(Q^{2}\right)$. These were parametrized in [ANS 89] in a very simple way by putting

$$
I_{p}\left(Q^{2}\right)=2 M^{2} \Gamma_{1, A s}^{p}\left[\frac{1}{Q^{2}+\mu^{2}}-\frac{c_{p} \mu^{2}}{\left(Q^{2}+\mu^{2}\right)^{2}}\right]
$$

where

$$
c_{p}=1+\frac{1}{8}\left(\frac{\mu^{2}}{M^{2}}\right) \frac{\kappa_{p}^{2}}{\Gamma_{1, A s}^{p}}
$$

and $\mu$ is a parameter setting the scale for the $Q^{2}$ variation. By vector dominance arguments it was suggested that $\mu^{2} \simeq m_{\rho}^{2}$. In $(4.2 .30,31) \Gamma_{1, A s}^{p}$ is taken as a constant, the limit of $\Gamma_{1}^{p}\left(Q^{2}\right)_{A s}$ as $Q^{2} \rightarrow \infty$.

Equation (4.2.30) used in (4.2.27) provides a formula for $\Gamma_{1}^{p}\left(Q^{2}\right)$ for all $Q^{2}$

$$
\Gamma_{1}^{p}\left(Q^{2}\right)=\frac{\Gamma_{1, A s}^{p}}{1+\mu^{2} / Q^{2}}\left[1-\frac{c_{p} \mu^{2}}{Q^{2}+\mu^{2}}\right]
$$

and equating this to the EMC result at $\left\langle Q^{2}\right\rangle=10.7(\mathrm{GeV} / \mathrm{c})^{2}$ yields $\Gamma_{1, A s}^{p} \simeq 1.2 \Gamma_{E M C}^{p}$, i.e some $20 \%$ larger than the EMC result (4.2.19). However the $Q^{2}$-dependence implied by (4.2.32) is too rapid and was later shown to contradict the $Q^{2}$-dependence of the data by 1.5 standard deviation [ASH 89].

More recently Burkert and Ioffe [BUR 92, 93] have argued that the contribution from resonance production has a strong $Q^{2}$-dependence for small $Q^{2}$ and then drops rapidly with $Q^{2}$ and that this should be substracted out before parametrizing the smooth large $Q^{2}$ behaviour. The behaviour of the resonance contribution is shown in Fig. 4.5. Equation (4.2.30) is then modified to

$$
I_{p}\left(Q^{2}\right)=I_{r e s}^{p}\left(Q^{2}\right)+2 M^{2} \Gamma_{1, A s}^{p}\left[\frac{1}{Q^{2}+\mu^{2}}-\frac{c_{p} \mu^{2}}{\left(Q^{2}+\mu^{2}\right)^{2}}\right]
$$

where now

$$
c_{p}=1+\frac{1}{2}\left(\frac{\mu^{2}}{M^{2}}\right) \frac{1}{\Gamma_{1, A s}^{p}}\left[\frac{\kappa_{p}^{2}}{4}+I_{\text {res }}^{p}(0)\right] .
$$

For the $\Delta\left(N^{*}(1238)\right)$ contribution they find [BUR 92]

$$
I_{\Delta}^{p}(0)=-0.78
$$

and including all resonances up to mass $1.8 \mathrm{GeV}$ [BUR 93]

$$
I_{\text {res }}^{p}(0)=-1.03 \text {. }
$$

Bearing in mind that $\kappa_{p}^{2} / 4=0.80$ we see that the resonance contribution has an important effect on the value of $c_{p}$. 
With this revised estimate of $c_{p}$, and using $\mu=m_{\rho}$, (4.2.32) suggests that $\Gamma_{1, A s}^{p}$ is about $8 \%$ larger than the EMC result. It appears that the $Q^{2}$-variation implied by $(4.2 .32)$ might just be compatible with the EMC data divided into two bins with $\left\langle Q^{2}\right\rangle=4.8$ and $\left\langle Q^{2}\right\rangle=17.2(\mathrm{GeV} / \mathrm{c})^{2}$.

For purposes of comparison with the QCD sum rule approach, let us expand (4.2.32) in inverse powers of $Q^{2}$ (we ignore $I_{\text {res }}\left(Q^{2}\right)$ at large $Q^{2}$ ):

$$
\Gamma_{1}^{p}\left(Q^{2}\right)=\Gamma_{1, A s}^{p}\left[1-\frac{\left(1+c_{p}\right) \mu^{2}}{Q^{2}}+\cdots\right] .
$$

The leading term in the higher twist correction is then

$$
\delta \Gamma_{1}^{p}\left(Q^{2}\right)=-\frac{\left(1+c_{p}\right) \mu^{2} \Gamma_{1, A s}^{p}}{Q^{2}} \approx-\frac{0.12(\mathrm{GeV} / \mathrm{c})^{2}}{Q^{2}} .
$$

In summary the higher twist corrections, estimated on the basis of the GDH sum rule, are likely to be less than $8 \%$ for the EMC experiment.

b) The QCD sum rule approach

In the usual operator product approach explained in Section 5 one keeps only the operators of lowest twist $\tau\left(\tau=2\right.$ for the study of $\left.g_{1}\left(x, Q^{2}\right)\right)$. The approach of [BAL 90], based on the QCD sum rule paper of Shuryak and Vainshtein [SHU 82], is a natural extension in which one studies also the operators of twist 4 and in which target mass corrections are taken into account. The problem is that one has to estimate certain matrix elements of gluon and quark condensates and certain correlators of quark and gluon operators. The latter is done assuming pseudoscalar meson dominance.

The leading target mass correction $\delta \Gamma_{1, T}$ to $\Gamma_{1}^{p, n}$ involves the third moment of $g_{1}^{p, n}$ and is given by [BAL 90, 93]

$$
\delta \Gamma_{1, T}^{p, n}=\frac{2 M^{2}}{9 Q^{2}} \int_{0}^{1} d x x^{2} g_{1}^{p, n}\left(x, Q^{2}\right)
$$

which yields

$$
\delta \Gamma_{1, T}^{p} \approx \frac{0.003 M^{2}}{Q^{2}}
$$

which is totally negligible in the EMC experiment.

The higher twist corrections require an estimate of the nucleon matrix elements of certain twist-4 $\left(\tau_{4}\right)$ operators. The contribution to $\Gamma_{1}^{p}$ involves flavour singlet $(\mathrm{S})$ and non-singlet (NS) operators:

$$
\delta \Gamma_{1, \tau_{4}}^{p}=-\frac{1}{6 Q^{2}}\left\{\frac{5}{3}\left\langle\left\langle O^{S}\right\rangle\right\rangle+\left\langle\left\langle O^{N S}\right\rangle\right\rangle\right\}
$$

where [BAL 90, 93]

$$
\langle\langle O\rangle\rangle=\frac{8}{9}\left[\langle\langle U\rangle\rangle-\frac{M^{2}}{2}\langle\langle V\rangle\rangle\right]
$$

and $\langle\langle U\rangle\rangle,\langle\langle V\rangle\rangle$ are the reduced matrix elements of operator $U_{\mu}$ and $V_{\mu \nu, \sigma}$, defined by

$$
\left\langle P, S\left|U_{\mu}\right| P, S\right\rangle=2 M S_{\mu}\langle\langle U\rangle\rangle
$$

and

$$
\left\langle P, S\left|V_{\mu \nu, \sigma}\right| P, S\right\rangle=2 M\langle\langle V\rangle\rangle\left\{\left(S_{\mu} P_{\nu}-S_{\nu} P_{\mu}\right) P_{\sigma}\right\}_{S_{\nu \sigma}}
$$


where $S_{\nu \sigma}$ means symmetrization with respect to $\nu, \sigma$.

The actual operators are :

$$
\begin{aligned}
U_{\mu}^{S / N S} & =g\left[\bar{u} \widetilde{\boldsymbol{G}}_{\mu \nu} \gamma^{\nu} u \pm \bar{d} \widetilde{\boldsymbol{G}}_{\mu \nu} \gamma^{\nu} d\right] \\
V_{\mu \nu, \sigma}^{S / N S} & =g\left\{\bar{u} \widetilde{\boldsymbol{G}}_{\mu \nu} \gamma_{\sigma} u \pm \bar{d} \widetilde{\boldsymbol{G}}_{\mu \nu} \gamma_{\sigma} d\right\}_{S_{\nu \sigma}}
\end{aligned}
$$

where $\widetilde{\boldsymbol{G}}_{\mu \nu}$ is the matrix

$$
\widetilde{\boldsymbol{G}}_{\mu \nu}=\frac{1}{2} \varepsilon_{\mu \nu \alpha \beta} G_{a}^{\alpha \beta}\left(\frac{\boldsymbol{\lambda}_{a}}{2}\right) .
$$

It is a non-trivial matter, using sum-rule techniques, to estimate the nucleon matrix elements of these operators and there is some uncertainty about the results. Moreover it is claimed [IOF 92] that the handling of the singlet is incorrect since the axial anomaly (to be discussed in Section 6) was not taken into account and certain correlators were estimated using pseudoscalar dominance by $\pi$ and $\eta$ which is inappropriate to the singlet case. In addition note that there are errors in [BAL 90] which are corrected in [BAL 93]. In their corrected papers [BAL 93] one has

$$
\begin{aligned}
& \delta \Gamma_{1, \tau_{4}}^{p}=-\frac{(0.02 \pm 0.013)(\mathrm{GeV} / \mathrm{c})^{2}}{Q^{2}} \\
& \delta \Gamma_{1, \tau_{4}}^{n}=-\frac{(0.005 \pm 0.003)(\mathrm{GeV} / \mathrm{c})^{2}}{Q^{2}}
\end{aligned}
$$

These corrections are much smaller than those obtained from the GDH sum rule (4.2.38) and are quite negligible for the EMC experiment.

c) Summary on higher twist effects in the EMC experiment

There is a significant difference between the size of these effects as calculated on the basis of the GDH sum rule (4.2.38) and on the basis of QCD sum rules (4.2.48). In both cases, however, the effects for the EMC experiment are negligible.

We shall thus proceed to analyse the theoretical implications of the EMC result, which gives a value for $a_{0}$ compatible with zero. In the following we shall use the value given in (4.2.22).

\subsection{The EMC result: implications in the Naive Parton Model}

In Appendix $\mathrm{B}$ we show how to express the protonic matrix element of the axial vector current of a quark field of definite flavour in terms of the polarized parton distributions, in the framework of the Naive Parton Model.

We have

$$
\left\langle P, S\left|\bar{\psi}_{f}(0) \gamma_{\mu} \gamma_{5} \psi_{f}(0)\right| P, S\right\rangle=2 M S_{\mu} \int_{0}^{1} d x\left[\Delta q_{f}(x)+\Delta \bar{q}_{f}(x)\right] .
$$

It follows from $(4.2 .3,5,7$ and 8$)$ that

$$
\begin{gathered}
a_{3}=\int_{0}^{1} d x[\Delta u(x)+\Delta \bar{u}(x)-\Delta d(x)-\Delta \bar{d}(x)] \\
a_{8}=\frac{1}{\sqrt{3}} \int_{0}^{1} d x[\Delta u(x)+\Delta \bar{u}(x)+\Delta d(x)+\Delta \bar{d}(x)-2 \Delta s(x)-2 \Delta \bar{s}(x)]
\end{gathered}
$$

and

$$
a_{0}=\Delta \Sigma \equiv \int_{0}^{1} d x \Delta \Sigma(x)
$$


where

$$
\Delta \Sigma(x) \equiv \Delta u(x)+\Delta \bar{u}(x)+\Delta d(x)+\Delta \bar{d}(x)+\Delta s(x)+\Delta \bar{s}(x) .
$$

Notice that (4.3.2-4) inserted into (4.2.1) agree with (4.1.1).

If, as is at first sight not an unreasonable assumption, one assumes that one can neglect the strange quark contribution so that $a_{0} \simeq \sqrt{3} a_{8}$ one is led to the Ellis-Jaffe sum rule [ELL 74] mentioned in Section 4 which gives a much larger value for $\Gamma_{1}^{p}$ than found by the EMC:

$$
\left(\Gamma_{1}^{p}\right)_{E J}=\frac{1}{12}\left\{a_{3}+\frac{5}{\sqrt{3}} a_{8}\right\} \simeq 0.188 \pm 0.004
$$

where we have used $(4.2 .14,16$ and 21$)$.

\subsubsection{The "spin crisis in the Parton Model"}

Let us consider now the physical significance of $\Delta \Sigma(x)$. Since $q^{ \pm}(x)$ count the number of quarks of momentum fraction $x$ with spin component $\pm \frac{1}{2}$ along the direction of motion of the proton (let us call this the $z$-direction), the total contribution to $J_{z}$ coming from a given flavour quark is

$$
\begin{aligned}
S_{z} & =\int_{0}^{1} d x\left\{\left(\frac{1}{2}\right) q^{+}(x)+\left(-\frac{1}{2}\right) q^{-}(x)\right\} \\
& =\frac{1}{2} \int_{0}^{1} d x \Delta q(x) .
\end{aligned}
$$

It follows that

$$
a_{0}=2 S_{z}^{q u a r k s}
$$

where $S_{z}^{q u a r k s}$ is the contribution to $J_{z}$ from the spin of all quarks and antiquarks.

Now in the Naive Parton Model $\boldsymbol{p}_{\perp}=0$ and all quarks move parallel to the parent hadron, i.e. for a quark of momentum $\boldsymbol{p}, \boldsymbol{p}=x \boldsymbol{P}$. Hence any orbital angular momentum carried by the quarks is perpendicular to $\boldsymbol{P}$ and thus does not contribute to $J_{z}$. In addition it is assumed that the gluons are unpolarized, for reasons explained in Section 4.3.2. Hence, in the Naive Parton Model, one expects for a proton of helicity $+1 / 2$ :

$$
S_{z}^{q u a r k s}=J_{z}=1 / 2 .
$$

We stress that this ignores $\boldsymbol{p}_{\perp}$ effects and assumes only quark and antiquark constituents are polarized.

From (4.3.8) and (4.2.22) we get instead of the value $1 / 2$ of (4.3.9)

$$
\left(S_{z}^{\text {quarks }}\right)_{\text {Exp }}=0.03 \pm 0.06 \pm 0.09
$$

It was this highly unexpected result which was termed the "spin crisis in the Parton Model" [LEA 88].

\subsubsection{The angular momentum sum rule}

In a more general framework, if we allow for the existence of parton transverse momentum and for the possibility that the gluons are polarized, (4.3.9) generalizes to the angular momentum sum rule

$$
J_{z}=S_{z}^{\text {quarks }}+S_{z}^{\text {gluons }}+L_{z}^{\text {partons }}=1 / 2
$$


and the question then arises as to whether the result (4.3.10) is as surprising as it at first seems. On the one hand models of the static properties of the baryons always have $S$-wave ground states of quarks only, so in these there is no $S_{z}^{\text {gluons }}$ nor $L_{z}^{\text {partons }}$. But these models deal with constituent quarks whereas the Parton Model involves current quarks.

Unfortunately nobody knows the precise connection between these so it is perhaps unjustified to make assertions about partons on the basis of constituent models. However, Lipkin [LIP 90] has given an argument based on partons, which emphasizes how peculiar it is that $S_{z}^{\text {quarks }}$ is so small. We present a simplified version of his treatment. The key point is that the $S U(6)$ prediction for $g_{A}$, namely $5 / 3$, is really quite close to the measured value (4.2.16), so that the proton wave function cannot be too different from the $S U(6)$ wave function. Thus if we write for this proton state

$$
|p\rangle=\cos \theta|S U(6)\rangle+\sin \theta|\psi\rangle
$$

where $|\psi\rangle$ is a state orthogonal to $|S U(6)\rangle$, the latter being a state in which the valence partons are in an $S U(6)$ configuration and anything else present has zero orbital, and zero total spin, angular momentum, then consideration of the contribution to $g_{A}$ from various possible plausible states $|\psi\rangle$, each having different amounts of spin carried by the valence quarks and by any other constituents, leads to the bound $\sin ^{2} \theta \leq 3 / 16$. On the other hand in the $S U(6)$ state, $J_{z}=S_{z}^{q u a r k s}=1 / 2$, so that requiring that

$$
\frac{1}{2} \cos ^{2} \theta+\left(S_{z}^{\text {quarks }}\right)_{\psi} \sin ^{2} \theta \simeq 0
$$

implies the large value

$$
\left|S_{z}^{q u a r k s}\right|_{\psi} \gtrsim 2
$$

Given the complexity of the state $|\psi\rangle$ that this implies, it is then hard to understand why the proton is the only stable state with $J=1 / 2, I=1 / 2$.

We shall be able to clarify this question a little more in Section 5 when we consider the surprising feature that QCD induces a $Q^{2}$-dependence in $S_{z}^{\text {gluons }}$ and $L_{z}^{\text {partons }}$. This will force us to rethink our interpretation of the physical meaning of the matrix element of $J_{5 \mu}^{0}$.

\subsubsection{Trouble with the strange quark}

There is another difficulty associated with the results that follow from analysing the EMC and hyperon data in terms of the Naive Parton Model.

We can use the numerical values (4.2.16, 21 and 22) to solve, via (4.2.14) and (4.3.2, $3,4)$, for the contributions of quarks and antiquarks of a given flavour. One finds:

$$
\begin{aligned}
& \Delta u \equiv \int_{0}^{1} d x[\Delta u(x)+\Delta \bar{u}(x)]=0.79 \pm 0.03 \pm 0.04 \\
& \Delta d \equiv \int_{0}^{1} d x[\Delta d(x)+\Delta \bar{d}(x)]=-0.47 \pm 0.03 \pm 0.04 \\
& \Delta s \equiv \int_{0}^{1} d x[\Delta s(x)+\Delta \bar{s}(x)]=-0.26 \pm 0.06 \pm 0.09 .
\end{aligned}
$$

But Preparata and Soffer [PRE 88] suggested that one can bound the strange quark contribution using the fact that, manifestly,

$$
|\Delta s(x)| \leq s(x)
$$


and the knowledge of the behaviour of $s(x)$ as determined from deep inelastic neutrino experiments.

There is an unjustified step in the analysis of [PRE 88] which can be modified [LEA $88 \mathrm{~b}$ ], and the argument is not rigorous since a specific form for $\Delta s$ is assumed. Nonetheless the bound is unlikely to be far from the truth. It yields

$$
|\Delta s|=\left|\int_{0}^{1} d x[\Delta s(x)+\Delta \bar{s}(x)]\right| \leq 0.072 \pm 0.030
$$

which is roughly a factor of three smaller than the value given in (4.3.17).

We have to conclude that the analysis based on the Naive Parton Model or the Operator Product Expansion with free fields is in difficulty.

\subsection{Analysis of $\Gamma_{1}$ using the QCD Improved Parton Model}

The analysis in Sections 4.1-3 was based on the Naive Parton Model which emerges from the Operator Product Expansion when all fields are treated as free fields. An immediate question is whether the difficulties we encountered can be removed by the inclusion of perturbative QCD corrections. In this Section we shall use the QCD-improved expressions which will be derived in Section 5 and demonstrate that the corrections do not help significantly. We shall also discuss the Bjorken sum rule.

\subsubsection{The operator product expansion for $\boldsymbol{\Gamma}_{\mathbf{1}}^{p}$}

As is well known, QCD corrections induce a logarithmic $Q^{2}$-dependence which breaks Bjorken scaling. As is outlined in Section 5.2 the expression (4.2.1) is modified to

$$
\Gamma_{1}^{p}\left(Q^{2}\right)=\frac{1}{12}\left\{\left(a_{3}+\frac{1}{\sqrt{3}} a_{8}\right) E_{N S}\left(Q^{2}\right)+\frac{4}{3} a_{0} E_{S}\left(Q^{2}\right)\right\}
$$

where the coefficient functions $E_{N S}$ and $E_{S}$ have perturbative expansions [KOD 79] recently extended to order $\alpha_{s}^{2}$ and $\alpha_{s}^{3}$ respectively [LAR 91, 94]

$$
\begin{gathered}
E_{N S}\left(Q^{2}\right)=1-\frac{\alpha_{s}}{\pi}-\left(\begin{array}{l}
3.58 \\
3.25
\end{array}\right)\left(\frac{\alpha_{s}}{\pi}\right)^{2}-\left(\begin{array}{l}
20.22 \\
13.85
\end{array}\right)\left(\frac{\alpha_{s}}{\pi}\right)^{3} \\
E_{S}\left(Q^{2}\right)=1-\left(\begin{array}{c}
0.333 \\
0.040
\end{array}\right)\left(\frac{\alpha_{s}}{\pi}\right)-\left(\begin{array}{r}
1.10 \\
-0.07
\end{array}\right)\left(\frac{\alpha_{s}}{\pi}\right)^{2}
\end{gathered}
$$

where $\alpha_{s}=\alpha_{s}\left(Q^{2}\right)$ and where the upper and lower figures refer to $N_{f}=3$ or 4 respectively. Strictly speaking the coefficients of $a_{3}$ and $a_{8}$ are only equal in the case of massless quarks, which is assumed in the calculation of (4.4.2). These perturbative corrections will be important in analysing the new data at lower $Q^{2}$, to be discussed in Section 4.5.2. In the notation of Section 5.2 we have $E_{N S}=E_{1,1}^{1}=E_{1,3}^{1} ; E_{S}=E_{1, \psi}^{1}$.

Once interactions are allowed the operators and currents have to be renormalized and their matrix elements will, in general, depend upon the subtraction point or renormalization scale $\mu$. For a conserved current, however, the matrix elements are independent of the renormalization scale. These matters were mentioned in Section 4.2 and will be taken up again in Section 6 . The important point to remember is that $a_{3}$ and $a_{8}$ are independent of $\mu$, whereas $a_{0}$ depends upon $\mu$. 
If we choose $\mu^{2}=Q^{2}$ then, in leading logarithmic approximation (LLA), the expressions $(4.3 .2-4)$ for the $a_{j}$ in terms of quark densities $\Delta q(x)$ are simply modified by the replacement

$$
\Delta q(x) \rightarrow \Delta q\left(x ; Q^{2}\right)
$$

where the $Q^{2}$-evolution is controlled by the spin dependent Altarelli-Parisi equations [ALT 77].

For the EMC experiment the mean value of $Q^{2}$ is about $10.7(\mathrm{GeV} / \mathrm{c})^{2}$ which corresponds to $\alpha_{s}\left(Q^{2}\right) \simeq 0.24$ for 4 flavours. The leading correction term is of order $8 \%$ in (4.4.2) and even smaller in (4.4.3 and we cannot expect a dramatic change in the values quoted in (4.2.22). Indeed one finds

$$
a_{0}=0.17 \pm 0.12 \pm 0.17
$$

to be compared with the naive expectation $a_{0} \simeq 1$. The values quoted in (4.3.15-17) become, for $Q^{2} \simeq 10(\mathrm{GeV} / \mathrm{c})^{2}$

$$
\begin{aligned}
\Delta u & =0.82 \pm 0.03 \pm 0.04 \\
\Delta d & =-0.44 \pm 0.03 \pm 0.04 \\
\Delta s & =-0.21 \pm 0.06 \pm 0.09
\end{aligned}
$$

which are close to the values given in (4.3.15-17) ए.

Thus the straightforward perturbative QCD corrections to (4.2.1) in no way alleviate the problem, and the value of $\Delta s$ is still surprisingly large compared with (4.3.19).

\subsection{The new experiments: neutron data and the Bjorken sum rule}

The operators $J_{5 \mu}^{0}$ and $J_{5 \mu}^{8}$ that give rise to the terms $a_{0}$ and $a_{8}$ in (4.4.1) are invariant under isotopic spin rotations. Thus in going from proton matrix elements to neutron matrix elements they remain unchanged. $J_{5 \mu}^{3}$, on the other hand, transforms like the 3rd component of an isotopic spin triplet and therefore changes sign. It follows that the Bjorken sum rule

$$
\int_{0}^{1} d x\left[g_{1}^{p}\left(x, Q^{2}\right)-g_{1}^{n}\left(x, Q^{2}\right)\right]=S_{B j}\left(Q^{2}\right)
$$

where $S_{B j}\left(Q^{2}\right)$ is the theoretical value of the Bjorken sum rule, given by

$$
\begin{aligned}
S_{B j}\left(Q^{2}\right) & =\frac{a_{3}}{6}\left\{1-\frac{\alpha_{s}}{\pi}-\left(\begin{array}{l}
3.58 \\
3.25
\end{array}\right)\left(\frac{\alpha_{s}}{\pi}\right)^{2}-\left(\begin{array}{c}
20.22 \\
13.85
\end{array}\right)\left(\frac{\alpha_{s}}{\pi}\right)^{3} \cdots\right\} \\
& =(0.2096 \pm 0.0005)\left\{1-\frac{\alpha_{s}}{\pi}-\left(\begin{array}{c}
3.58 \\
3.25
\end{array}\right)\left(\frac{\alpha_{s}}{\pi}\right)^{2}-\left(\begin{array}{l}
20.22 \\
13.85
\end{array}\right)\left(\frac{\alpha_{s}}{\pi}\right)^{3} \ldots\right\}
\end{aligned}
$$

holds on fundamental grounds. Note that the fact that $a_{3}$ is independent of $Q^{2}$ is linked to the conservation of the non-singlet axial current and is discussed more fully in Section 6.1. The upper and lower figures in (4.5.2) refer to $N_{f}=3$ or 4 respectively.

1) Note added in proof: An analysis of all the proton data at $Q^{2}=10(\mathrm{GeV})^{2}$ [ADA 94] alters (4.2.19) to $\Gamma_{1}^{p}=0.142 \pm 0.008 \pm 0.011$ and consequently (4.4.5) becomes (notice that in [ADA 94] $\alpha_{s} \simeq 0.23$ is used) $a_{0}=0.27 \pm 0.08 \pm 0.10$, still uncomfortably far from $a_{0}=1$. 
The Ellis-Jaffe sum rule value (4.3.6), $\Gamma_{1}^{p} \simeq 0.188$, would imply, via $(4.5 .1,2)$ at $Q^{2}=10.7(\mathrm{GeV} / \mathrm{c})^{2}$

$$
\Gamma_{1}^{n} \equiv \int_{0}^{1} d x g_{1}^{n}\left(x, Q^{2}\right) \simeq-0.0003
$$

whereas the measured EMC value of $\Gamma_{1}^{p}$ gives hope of a somewhat more sizeable neutron result:

$$
\Gamma_{1}^{n} \simeq-0.06 \quad \text { at } \quad Q^{2}=10.7(\mathrm{GeV} / \mathrm{c})^{2}
$$

\subsubsection{The new experiments on deuterium and ${ }^{3} \mathrm{He}$}

The first experiments to measure $g_{1}^{n}(x)$ have now begun to report results. At CERN the Spin Muon Collaboration (SMC) have used a polarized deuterium target at an average $Q^{2}$ of $4.6(\mathrm{GeV} / \mathrm{c})^{2}$ [ADE 93]. At SLAC the E142 group uses a polarized ${ }^{3} \mathrm{He}$ target at $\left\langle Q^{2}\right\rangle=2.0(\mathrm{GeV} / \mathrm{c})^{2}[\mathrm{ANT} 93]$. (For a combined analysis of the data see [ADE 94]).

The SMC data on the deuteron asymmetry $A_{1}^{d}(x)$ at $Q^{2}=4.6(\mathrm{GeV} / \mathrm{c})^{2}$ are shown in Fig. 4.6, and in Fig. 4.7 the data on $x g_{1}^{d}(x)$. The E142 data on the neutron asymmetry $A_{1}^{n}(x)$ and on $g_{1}^{n}(x)$ at $Q^{2}=2(\mathrm{GeV} / c)^{2}$ are shown in Fig. 4.8. The neutron data were extracted as explained in Section 2.1.4 using the EMC proton data.

Soon after the appearance of the new results some rather dramatic claims were made that the very fundamental Bjorken sum rule had been violated. In retrospect these incorrect assertions arose because of too naive a comparison of the new data with the EMC proton data at $10.7(\mathrm{GeV} / \mathrm{c})^{2}$. A more careful analysis is necessary, as emphasized by Ellis and Karliner [ELL 93] and by Close and Roberts [CLO 93] and Altarelli, Nason, Ridolfi [ALT 94].

The main issues are the following:

1) The extraction of $g_{1}^{n}\left(x, Q^{2}\right)$ from nuclear data at some $\left\langle Q^{2}\right\rangle$, as explained in Section 2.1.4, requires a knowledge of $g_{1}^{p}\left(x, Q^{2}\right)$ at the same mean $Q^{2}$. It is unjustified to naively combine the EMC data at $\left\langle Q^{2}\right\rangle=10.7(\mathrm{GeV} / \mathrm{c})^{2}$ with the deuterium data at 4.6 and the ${ }^{3} \mathrm{He}$ data at $2.0(\mathrm{GeV} / \mathrm{c})^{2}$.

There is no absolutely safe way to overcome this difficulty. It does seem, however, that the values of the asymmetries at fixed $x$, show no significant variation with $Q^{2}$ where it has been possible to examine this. On the other hand the variation of $F_{2}\left(x, Q^{2}\right)$ with $Q^{2}$ has been much studied.

In the improved analysis, therefore, $g_{1}^{p}\left(x, Q^{2}\right)$ is calculated at the required $Q^{2}$ by using

$$
g_{1}^{p}\left(x, Q^{2}\right)=\frac{A_{\|}^{p}}{D} \frac{F_{2}^{p}\left(x, Q^{2}\right)}{2 x\left[1+R^{p}\left(x, Q^{2}\right)\right]}
$$

and assuming $A_{\|}^{p}$ independent of $Q^{2}$. The resulting dependence of $g_{1}^{p}\left(x, Q^{2}\right)$ upon the mean $Q^{2}$ is shown in Fig. 4.9 taken from [ELL 93]. In (4.5.3) values of $F_{2}^{p}$ were taken from the NMC data [AMA 92] and of $R^{p}$ from the SLAC data [WHI 90]. These $g_{1}^{p}\left(x, Q^{2}\right)$ yield, [ELL 93],

$$
\begin{aligned}
\Gamma_{1}^{p}\left[2(\mathrm{GeV} / \mathrm{c})^{2}\right] & =0.124 \pm 0.013 \pm 0.019 \\
\Gamma_{1}^{p}\left[4.6(\mathrm{GeV} / \mathrm{c})^{2}\right] & =0.125 \pm 0.013 \pm 0.019
\end{aligned}
$$

Figure 4.10 shows the $g_{1}^{n}\left(x, Q^{2}\right)$ extracted from the nuclear data on this basis.

2) The extrapolation of $g_{1}^{n}\left(x, Q^{2}\right)$ to $x=1$ and $x=0$ in order to compute the first moment $\Gamma_{1}^{n}$ requires some care. 
The SMC experiment at $\left\langle Q^{2}\right\rangle=4.6(\mathrm{GeV} / \mathrm{c})^{2}$ covers the range $0.006 \leq x \leq 0.6$. They find

$$
\int_{0.006}^{0.6} g_{1}^{d}(x) d x=0.024 \pm 0.020 \pm 0.014
$$

The extrapolation to $x=0$ is taken to be of the reasonable form $g_{1}^{d} \sim x^{-\alpha}$ with $-0.5 \leq \alpha \leq 0$, in accord with the discussion in Section 4.2.3 and compatible with Eq. (4.2.23). This leads to an estimate of $-0.003 \pm 0.003$ for the integral from 0 to 0.006 .

The extrapolation to $x=1$ is based on the fact that the asymmetry is bounded, $|A| \leq 1$, and leads to the estimate $0.002 \pm 0.004$ for the integral from 0.6 to 1 .

The result is, per nucleon,

$$
\Gamma_{1}^{d}\left[Q^{2}=4.6(\mathrm{GeV} / \mathrm{c})^{2}\right]=0.023 \pm 0.020 \pm 0.015
$$

Note that using $(2.1 .55),(4.4 .2,3)$ and $(4.2 .14)$ we have (taking $N_{f}=4$ )

$$
\Gamma_{1}^{d}=\frac{\left(1-1.5 \omega_{D}\right)}{12}\left\{\frac{1}{3}(3 F-D)\left[1-\frac{\alpha_{s}}{\pi}-3.25\left(\frac{\alpha_{s}}{\pi}\right)^{2}\right]+\frac{4}{3} a_{0}\left[1-0.04 \frac{\alpha_{s}}{\pi}\right]\right\} .
$$

Taking $\alpha_{s}=0.28 \pm 0.03$ at $Q^{2}=4.6(\mathrm{GeV} / c)^{2}[\mathrm{NAS} 93]$ and using the values in (4.2.21), one obtains $\left(\omega_{D}=0.058\right)$

$$
a_{0}=0.09 \pm 0.20 \pm 0.15
$$

again consistent with zero and perfectly compatible with the value quoted in (4.4.5).

For $\Gamma_{1}^{n}$ [ELL 93] obtain a result very slightly different from the value quoted by the SMC because of the small change in $\Gamma_{1}^{p}$ shown in (4.5.4), i.e.

$$
\Gamma_{1}^{n}\left[4.6(\mathrm{GeV} / \mathrm{c})^{2}\right]=-0.076 \pm 0.037 \pm 0.046
$$

The E142 experiment at $\left\langle Q^{2}\right\rangle=2(\mathrm{GeV} / \mathrm{c})^{2}$ covers the range $0.03 \leq x \leq 0.6$. [ELL 93] have re-analysed the extrapolations to $x=0$ and $x=1$ using the same approach as in the SMC case and they estimate contributions to $\Gamma_{1}^{n}$ of $-0.006 \pm 0.006$ for the integral from 0 to 0.03 and $0.000 \pm 0.003$ for the region 0.6 to 1 . The result is

$$
\Gamma_{1}^{n}\left[2.0(\mathrm{GeV} / \mathrm{c})^{2}\right]=-0.028 \pm 0.006 \pm 0.009
$$

which differs somewhat from the value $-0.022 \pm 0.011$ quoted by E142.

\subsubsection{Tests of the Bjorken sum rule}

Using the values of $\Gamma_{1}^{p}$ given in (4.5.4) and of $\Gamma_{1}^{n}$ given in (4.5.9 and 10) one can now test the Bjorken sum rule, bearing in mind, however, that we have assumed that the asymmetry is $Q^{2}$-independent for the range involved. Before comparing with the data it is instructive to see to what extent the perturbative QCD corrections influence the expected value of the Bjorken sum rule, $S_{B_{j}}\left(Q^{2}\right)$ given by (4.5.2). We show below in Table 4.1 the values of $S_{B_{j}}\left(Q^{2}\right)$ at various $Q^{2}$ calculated to order $\alpha_{s}, \alpha_{s}^{2}$ and $\alpha_{s}^{3}$ starting from the central value 0.2096 without corrections. We have used values of $\alpha_{s}\left(Q^{2}\right)$ from [NAS 93] which differ somewhat from the values used in [ELL 93] and also from those used by the SMC group. We believe these represent a good compromise between the LEP and unpolarized DIS determinations which differ somewhat. 
Table 4.1 Values of $\alpha_{s}\left(Q^{2}\right)$ and of $S_{B_{j}}\left(Q^{2}\right)$ to various orders in perturbation theory

\begin{tabular}{|c|c|c|c|c|c|}
\hline & & & \multicolumn{3}{|c|}{$S_{B_{j}}\left(Q^{2}\right)=0.2096$ to zero order } \\
\cline { 4 - 6 }$Q^{2}$ & \multirow{2}{*}{$N_{f}$} & $\alpha_{s}\left(Q^{2}\right)$ & to $\mathcal{O}\left(\alpha_{s}\right)$ & to $\mathcal{O}\left(\alpha_{s}^{2}\right)$ & to $\mathcal{O}\left(\alpha_{s}^{3}\right)$ \\
\hline in $(\mathrm{GeV} / \mathrm{c})^{2}$ & & & & & \\
\hline 2 & 3 & $0.354 \pm 0.04$ & 0.186 & 0.176 & 0.170 \\
4.6 & 4 & $0.283 \pm 0.03$ & 0.191 & 0.185 & 0.183 \\
10.7 & 4 & $0.238 \pm 0.03$ & 0.194 & 0.190 & 0.189 \\
\hline
\end{tabular}

We see that at $Q^{2}=2$ the corrections are very important, $\simeq 20 \%$, and that even at $Q^{2}$ $=10.7$ they are not negligible, $\simeq 10 \%$.

We come now to the comparison between the experimental and theoretical values of the Bjorken sum rule. In allowing for target mass corrections and higher twist corrections as discusses in Section 4.2.4 we must recall that the GDH and QCD sum rule approach give quite different estimates for the higher twist corrections.

In general terms one has

$$
\int_{0}^{1} d x\left[g_{1}^{p}\left(x, Q^{2}\right)-g_{1}^{n}\left(x, Q^{2}\right)\right]=S_{B_{j}}\left(Q^{2}\right)+\delta S_{\tau 4}^{B j}+\delta S_{T}^{B j} .
$$

The target mass correction given in (4.2.40) is negligible for the proton and an estimate for the neutron is much smaller [ELL 93] so we can ignore $\delta S_{T}^{B j}$ even at $Q^{2}=2$.

In the QCD sum rule approach one has, from (4.2.48)

$$
\left.\delta S_{\tau 4}^{B j}\right|_{Q C D \text { sum rules }} \approx-\frac{(0.015 \pm 0.013)(\mathrm{GeV} / \mathrm{c})^{2}}{Q^{2}} .
$$

In order to estimate the higher twist corrections in the GDH sum rule approach let us rewrite $(4.2 .37)$ as

$$
\Gamma_{1}^{p, n}\left(Q^{2}\right)=\Gamma_{1, A s}^{p, n}-\frac{\mu^{2}}{Q^{2}}\left[2 \Gamma_{1, A s}^{p, n}+\lambda_{p, n}\right]
$$

where

$$
\lambda_{p, n} \equiv \frac{1}{2}\left(\frac{\mu^{2}}{M^{2}}\right)\left[\frac{\kappa_{p, n}^{2}}{4}+I_{p, n}^{R e s}(0)\right] .
$$

Using the values [BUR 93]

$$
I_{p}^{\operatorname{Res}}(0)=-1.028 \quad I_{n}^{\operatorname{Res}}(0)=-0.829
$$

we find

$$
\begin{aligned}
\left.\delta S_{\tau 4}^{B j}\right|_{\text {GDH sum rule }} & =-\frac{\mu^{2}}{Q^{2}}\left\{2\left(\Gamma_{1}^{p}-\Gamma_{1}^{n}\right)_{A s}+\lambda_{p}-\lambda_{n}\right\} \\
& =-\frac{0.16(\mathrm{GeV} / \mathrm{c})^{2}}{Q^{2}}
\end{aligned}
$$

This appears to be a huge correction at small values of $Q^{2}$. However it is illegitimate to use (4.5.16) in a region where the resonance contribution $I_{\text {Res }}\left(Q^{2}\right)$ in (4.2.33) has not yet died out. The $(p-n)$ curve in Fig. 4.5 indicates that $I_{\text {Res }}$ is not negligible at $Q^{2}=2$ $(\mathrm{GeV} / \mathrm{c})^{2}$. Thus we should add a contribution

$$
\left.\frac{Q^{2}}{2 M^{2}} I_{\text {Res }}^{p-n}\left(Q^{2}\right)\right|_{Q^{2}=2} \simeq 0.05
$$


to the correction $(4.5 .16)$ at $Q^{2}=2(\mathrm{GeV} / \mathrm{c})^{2}$, yielding

$$
\left.\delta S^{B j}\right|_{\text {GDH sumrule }}=-0.03 \quad \text { at } \quad Q^{2}=2(\mathrm{GeV} / \mathrm{c})^{2} .
$$

At $Q^{2}=4.6(\mathrm{GeV} / \mathrm{c})^{2}$ it would appear to be safe to neglect $I_{\text {Res }}\left(Q^{2}\right)$ and to use just (4.5.16). However the corrections do seem surprisingly large. We are unable to estimate the errors in (4.5.16 and 18).

In Table 4.2 we compare the experimental value of the LHS of (4.5.11) with the theoretical value of the RHS using the results of Table 4.1 and with the two versions of the higher twist corrections.

Table 4.2 Test of the Bjorken sum rule

\begin{tabular}{|c|c|c|c|}
\hline & & \multicolumn{2}{|c|}{$S_{B_{j}}\left(Q^{2}\right)+\delta S_{T}^{B_{j}}+\delta S_{\tau 4}^{B_{j}}$} \\
\cline { 3 - 4 } & & & \\
$\left\langle Q^{2}\right\rangle$ & $\int_{0}^{1} d x\left[g_{1}^{p}\left(x, Q^{2}\right)-g_{1}^{n}\left(x, Q^{2}\right)\right]$ & QCD sum rule & GDH sum rule \\
\hline in $(\mathrm{GeV} / \mathrm{c})^{2}$ & & & 0.14 \\
\hline 2 & $0.152 \pm 0.014 \pm 0.021$ & $0.162 \pm 0.023$ & 0.15 \\
\hline 4.6 & $0.201 \pm 0.039 \pm 0.050$ & $0.180 \pm 0.017$ & \\
\hline
\end{tabular}

It is seen that there is absolutely no significant evidence for a failure of the sum rule. Clearly at the low value of $Q^{2}=2$ the higher twist effects help to make the agreement more impressive, but even without them there is no real contradiction, given the size of the experimental errors.

\section{The Operator Product Expansion (OPE)}

The fundamental understanding of the $Q^{2}$-behaviour of the moments in unpolarized DIS came originally from a study of the operator product expansion. Later it was discovered that the same results could be obtained in the QCD Improved Parton Model.

When it comes to the polarized case it has been claimed that the two approaches yield different results. We shall argue that this is not the case and claim that there is perfect agreement between the two methods.

We shall also clarify the situation with regard to $g_{2}(x)$ itself, to its first moment (the Burkhardt-Cottingham sum rule), and to its relationship with $g_{1}(x)$ (the EfremovLeader-Teryaev and the Wandzura-Wilczek sum rules), about which there are many misconceptions in the literature.

\subsection{General structure of the OPE}

It is well known, and can easily be deduced from the Feynman diagram Fig. 2.1, that the hadronic tensor $W^{\mu \nu}$ involved in the expression for the deep inelastic crosssection [see (2.1.1)] is given by the Fourier transform of the nucleon matrix elements of the commutator of electromagnetic currents $J_{\mu}(x)$ :

$$
W_{\mu \nu}(q ; P, S)=\frac{1}{2 \pi} \int d^{4} x e^{i q \cdot x}\left\langle P, S\left|\left[J_{\mu}(x), J_{\nu}(0)\right]\right| P, S\right\rangle
$$

where $S^{\mu}$ is the covariant spin vector specifying the nucleon state of momentum $P^{\mu}$. It is convenient to introduce an amplitude $T_{\mu \nu}$ which is closely related to the forward $T$-matrix 
element for Compton scattering of a virtual photon of 4-momentum $q$ and helicities $\lambda$ and $\lambda^{\prime}$. In the convention for the $\hat{T}$-operator

$$
\hat{S}=\hat{I}+i(2 \pi)^{4} \delta^{4}\left(P_{f}-P_{i}\right) \hat{T}
$$

one has

$$
\left\langle P, S ; q, \lambda^{\prime}|\hat{T}| P, S ; q, \lambda\right\rangle=4 \pi \alpha \varepsilon_{\mu}^{*}\left(\lambda^{\prime}\right) T^{\mu \nu} \varepsilon_{\nu}(\lambda)
$$

where

$$
T_{\mu \nu}(q ; P, S)=i \int d^{4} x e^{i q \cdot x}\left\langle P, S\left|T\left(J_{\mu}(x) J_{\nu}(0)\right)\right| P, S\right\rangle
$$

is given in terms of the time ordered product of the currents.

Both $W_{\mu \nu}$ and $T_{\mu \nu}$ may be split into parts symmetric $(S)$ or antisymmetric $(A)$ under $\mu \leftrightarrow \nu$, and one can show that

$$
W_{\mu \nu}^{(S, A)}=\frac{1}{\pi} \operatorname{Im} T_{\mu \nu}^{(S, A)}
$$

The symmetric part is independent of the spin vector $S^{\mu}$ and plays a rôle only in unpolarized scattering. We shall therefore concentrate on the antisymmetric part and we follow the treatment of Kodaira and his group [KOD 79].

The behaviour of $T_{\mu \nu}$ (and therefore $W_{\mu \nu}$ ) in the deep inelastic limit is controlled by the behaviour of the product of currents near the light cone $x^{2}=0$ and can be derived from Wilson's operator product expansion.

It is important to note that the expressions (5.1.1 and 3), for which the operator product approach can be utilised, only arise because of the fully inclusive nature of the deep inelastic reaction being considered. Indeed the starting point from which (5.1.1) can be derived is the expression

$$
W_{\mu \nu} \propto \sum_{X}\left\langle P, S\left|J_{\mu}\right| X\right\rangle\left\langle X\left|J_{\nu}\right| P, S\right\rangle
$$

which appears in the formula for the cross-section. Only if the sum is over all final states $|X\rangle$ does this reduce to (5.1.1).

The antisymmetric part of the Fourier transform of the operator product appearing in (5.1.3) is expanded in terms of local operators $R$ and coefficient functions $E$ in the form [KOD 79]:

$$
\begin{aligned}
i \int d^{4} x e^{i q \cdot x} T\left(J_{\mu}(x) J_{\nu}(0)\right) & =-i \sum_{n=1} \frac{\left[1-(-1)^{n}\right]}{2}\left(\frac{2}{Q^{2}}\right)^{n} q_{\mu_{1}} \ldots q_{\mu_{n-2}} \sum_{i} \delta_{i}\left\{\varepsilon_{\mu \nu \lambda \sigma} q^{\lambda} q_{\mu_{n-1}}\right. \\
& \times E_{1, i}^{n}\left(Q^{2}, g\right) R_{1, i}^{\sigma \mu_{1} \ldots \mu_{n-1}} \\
& +\left(\varepsilon_{\mu \rho \lambda \sigma} q_{\nu} q^{\rho}-\varepsilon_{\nu \rho \lambda \sigma} q_{\mu} q^{\rho}-q^{2} \varepsilon_{\mu \nu \lambda \sigma}\right) \frac{n-1}{n} \\
& \left.\times E_{2, i}^{n}\left(Q^{2}, g\right) R_{2, i}^{\lambda \sigma \mu_{1} \ldots \mu_{n-2}}\right\}
\end{aligned}
$$

where $g$ is the QCD coupling constant and where $i$ takes on the values $1,2 \ldots, 8, \psi, G$, the detailed significance of which will be explained below. The operators with $i=1, \ldots, 8$ transform like an $\mathrm{SU}(3)$ flavour octet. Those with labels $\psi$ or $G$ are flavour singlets.

The factors $\delta_{i}$ reflect the charge and isotopic spin structure of the currents. Taking for the electromagnetic current (in units of $e$ )

$$
J_{\mu}=\sum_{f} e_{f} \bar{\psi}_{f} \gamma_{\mu} \psi_{f} \quad f=u, d, s
$$


(a colour sum is, of course, implied), and bearing in mind that we are ultimately only interested in the current commutator that occurs in $W_{\mu \nu}$, one may take for the $\delta_{i}$

$$
\delta_{1}=\frac{1}{3} \quad \delta_{8}=\frac{1}{3 \sqrt{3}} \quad \delta_{\psi}=\delta_{G}=\frac{2}{9}
$$

and all other $\delta_{i}=0$.

Note that, as will be seen below, there does not exist an $R_{2}$-type operator with $n<2$. Nonetheless because of the explicit factor $(n-1)$ in (5.1.5) we have formally written a sum from $n=1$. Also there is no operator of the $R_{1, G}$ type for $n=1$. It should formally be regarded as zero for $n=1$ in the sum over $n$.

The leading twist operators occurring are of two types:

1) The set $R_{1, i}^{\sigma \mu_{1} \ldots \mu_{n-1}}$ of twist 2 .

For $i=1, \ldots, 8$ these are the flavour non-singlet operators

$$
R_{1, i}^{\sigma \mu_{1} \ldots \mu_{n-1}}=(i)^{n-1}\left\{\overline{\boldsymbol{\psi}} \gamma_{5} \gamma^{\sigma} D^{\mu_{1}} \ldots D^{\mu_{n-1}}\left(\frac{\boldsymbol{\lambda}_{i}}{2}\right) \boldsymbol{\psi}\right\}_{S} \quad(n \geq 1)
$$

where $S$ implies complete symmetrization in the indices $\sigma, \mu_{1}, \mu_{2}, \ldots, \mu_{n-1}$, the $\boldsymbol{\lambda}_{i}$ are the Gell-Mann $S U(3)$ flavour matrices and $D^{\mu}$ is the usual QCD covariant derivative. For $i=\psi$ or $G$ we have the flavour singlet operators

$$
\begin{aligned}
& R_{1, \psi}^{\sigma \mu_{1} \ldots \mu_{n-1}}=(i)^{n-1}\left\{\overline{\boldsymbol{\psi}} \gamma_{5} \gamma^{\sigma} D^{\mu_{1}} \ldots D^{\mu_{n-1}} \boldsymbol{\psi}\right\}_{S} \quad(n \geq 1) \\
& R_{1, G}^{\sigma \mu_{1} \ldots \mu_{n-1}}=(i)^{n-1} \operatorname{Tr}\left\{\varepsilon^{\sigma \alpha \beta \gamma} \boldsymbol{G}_{\beta \gamma} D^{\mu_{1}} \ldots D^{\mu_{n-2}} \boldsymbol{G}_{\alpha}^{\mu_{n-1}}\right\}_{S} \quad(n \geq 2)
\end{aligned}
$$

where $\boldsymbol{G}_{\mu \nu}$ is the usual matrix (in colour space) form of the gluon field tensor [see Eq. (6.3.5)].

2) The set $R_{2, i}^{\sigma \lambda \mu_{1} \ldots \mu_{n-2}}$ of twist 3 .

For $i=1, \ldots, 8$ these are the flavour non-singlet operators

$$
R_{2, i}^{\lambda \sigma \mu_{1} \ldots \mu_{n-2}}=(i)^{n-1}\left\{\overline{\boldsymbol{\psi}} \gamma_{5} \gamma^{\lambda} D^{\sigma} D^{\mu_{1}} \ldots D^{\mu_{n-2}}\left(\frac{\boldsymbol{\lambda}_{i}}{2}\right) \boldsymbol{\psi}\right\}_{S^{\prime}} \quad(n \geq 2)
$$

where $S^{\prime}$ implies antisymmetrization on $\lambda, \sigma$ and symmetrization on the indices $\mu_{1}, . ., \mu_{n-2}$.

For $i=\psi$ or $G$ we have flavour singlet operators

$$
\begin{aligned}
& R_{2, \psi}^{\lambda \sigma \mu_{1} \ldots \mu_{n-2}}=(i)^{n-1}\left\{\overline{\boldsymbol{\psi}} \gamma_{5} \gamma^{\lambda} D^{\sigma} D^{\mu_{1}} \ldots D^{\mu_{n-2}} \boldsymbol{\psi}\right\}_{S^{\prime}} \quad(n \geq 2) \\
& R_{2, G}^{\lambda \sigma \mu_{1} \ldots \mu_{n-2}}=(i)^{n-1} \operatorname{Tr}\left\{\varepsilon^{\sigma \alpha \beta \gamma} \boldsymbol{G}_{\beta \gamma} D^{\mu_{1}} \ldots D^{\mu_{n-2}} \boldsymbol{G}_{\alpha}^{\lambda}\right\}_{S^{\prime}} \quad(n \geq 2) .
\end{aligned}
$$

The above operators are often referred to as $R_{1, i}^{n}, R_{2, i}^{n}$ for brevity.

It should be noted that the above list of operators is a complete set of operators in the massless quark theory (if $m_{q} \neq 0$ there exists a further set of twist 3 operators). We shall not deal with these. They are discussed in [KOD 79].

The coefficient functions $E_{1, i}^{n}\left(Q^{2}, g\right), E_{2, i}^{n}\left(Q^{2}, g\right)$ are the Fourier transforms of the singular functions of $x$ that appear in the Operator Product Expansion and have to be calculated, using perturbative QCD, as a power series expansion in the coupling $g$. The factors in (5.1.5) are arranged so that for free fields, i.e. $g=0$, one has the simple results:

$$
\begin{aligned}
E_{1, i}^{n}\left(Q^{2}, g=0\right) & =1 & & E_{2, i}^{n}\left(Q^{2}, g=0\right)=1 \quad i=1, \ldots, 8 \\
E_{1, \psi}^{n}\left(Q^{2}, g=0\right) & =1 & & E_{2, \psi}^{n}\left(Q^{2}, g=0\right)=1 \\
E_{1, G}^{n}\left(Q^{2}, g=0\right) & =0 & & E_{2, G}^{n}\left(Q^{2}, g=0\right)=0 .
\end{aligned}
$$


Since gluons are electrically neutral it is no surprise to see that the operators involving the gluon field tensors $G_{\mu \nu}^{a}$ play no rôle when $g=0$.

In an interacting theory the $E$ 's are functions, $E_{1, i}^{n}\left(Q^{2} / \mu^{2} ; g\right)$ etc., of the renormalization scale $\mu^{2}$ and they satisfy standard renormalization group equations. The flavour octet operators are multiplicatively renormalized, but the $G$ and $\psi$ type singlet operators mix under renormalization, except for the case $n=1$. This is reflected in the $Q^{2}$-evolution of the coefficient functions (for details see [KOD 79]).

The proton matrix elements of these operators have the form:

$$
\begin{aligned}
\left\langle P, S\left|R_{1, i}^{\sigma \mu_{1} \ldots \mu_{n-1}}\right| P, S\right\rangle & =\frac{-2 M a_{n}^{i}}{n}\left\{S^{\sigma} P^{\mu_{1}} \ldots P^{\mu_{n-1}}\right\}_{S} \\
\left\langle P, S\left|R_{2, i}^{\lambda \sigma \mu_{1} \ldots \mu_{n-2}}\right| P, S\right\rangle & =M d_{n}^{i}\left(S^{\sigma} P^{\lambda}-S^{\lambda} P^{\sigma}\right) P^{\mu_{1}} \ldots P^{\mu_{n-2}},
\end{aligned}
$$

where the factors $a_{n}^{i}, d_{n}^{i}$ - essentially reduced matrix elements - reflect the unknown, nonperturbative aspect of the dynamics. Depending upon which current is involved, they may or may not depend upon the renormalization scale $\mu^{2}$, as is discussed later.

The connection with the simpler operators used in Section 4 is as follows. For $n=1$ we have

$$
\begin{gathered}
\left(R_{1, i}\right)_{\sigma}=\overline{\boldsymbol{\psi}}_{\gamma_{5}} \gamma_{\sigma}\left(\frac{\boldsymbol{\lambda}_{i}}{2}\right) \boldsymbol{\psi} \quad i=1, \ldots, 8 \\
=-J_{5 \sigma}^{i} \quad \text { of eq. }(4.2 .3)
\end{gathered}
$$

so that via (4.2.7) and (5.1.13)

$$
a_{i}=2 a_{1}^{i}
$$

Also, note that

$$
\begin{aligned}
\left(R_{1, \psi}\right)_{\sigma}= & \overline{\boldsymbol{\psi}} \gamma_{5} \gamma_{\sigma} \boldsymbol{\psi} \\
& =-J_{5 \sigma}^{0} \quad \text { of eq. (4.2.5) }
\end{aligned}
$$

so that via (4.2.8) and (5.1.13)

$$
a_{0}=a_{1}^{\psi}
$$

\subsection{Equations for the moments of $g_{1,2}\left(x, Q^{2}\right)$}

The expressions for the moments are obtained from the OPE in the standard fashion:

i) $g_{1,2}$ are related to the absorptive parts of forward scattering amplitudes for virtual Compton scattering;

ii) dispersion relations in the energy $\nu$ are written for these scattering amplitudes;

iii) for small enough $\nu$ the latter can be expanded in a power series in $\nu$ or, equivalently, in $1 / x$. Only even or odd powers appear because of the crossing symmetry of the scattering amplitudes. The coefficients in the power series are moments of the absorptive parts and thus of the scaling functions $g_{1,2}$;

iv) this power series in $1 / x$ is matched to the one which emerges from the OPE when the scalar products occurring in (5.1.5) are multiplied out.

The result is an expression for the moments of $g_{1,2}$ in terms of the factors $a_{n}^{i}, d_{n}^{i}$ and the coefficient functions $E_{1, i}^{n}$ and $E_{2, i}^{n}$. One finds [KOD 79] for the $n$-th moment:

$$
\int_{0}^{1} d x x^{n-1} g_{1}\left(x, Q^{2}\right)=\frac{1}{2} \sum_{i} \delta_{i} a_{n}^{i} E_{1, i}^{n}\left(Q^{2}, g\right) \quad n=1,3,5 \ldots
$$


and

$$
\int_{0}^{1} d x x^{n-1} g_{2}\left(x, Q^{2}\right)=\left(\frac{1-n}{2 n}\right) \sum_{i} \delta_{i}\left[a_{n}^{i} E_{1, i}^{n}\left(Q^{2}, g\right)-d_{n}^{i} E_{2, i}^{n}\left(Q^{2}, g\right)\right] \quad n=3,5,7 \ldots
$$

Note firstly that the Operator Product Expansion only gives information about the odd moments of $g_{1,2}\left(x, Q^{2}\right)$.

Note also the important feature that (5.2.2) only gives information about the moments of $g_{2}$ for $n \geq 3$. In the original literature (5.2.2) is given as holding also for $n=1$. That this is incorrect has been stressed by Jaffe [JAF 90], and we support his contention. Equation (5.2.2) thus gives no information about the first moment

$$
\Gamma_{2}\left(Q^{2}\right) \equiv \int_{0}^{1} d x g_{2}\left(x, Q^{2}\right)
$$

Let us now concentrate on the first moment, $n=1$, of $g_{1}$ in Eq. (5.2.1). Since, from (5.1.9), there does not exist a gluon operator for $n=1$, the first moment $\Gamma_{1}\left(Q^{2}\right)$ of $g_{1}$ does not receive a direct contribution from gluonic operators. In fact in the free theory, with $g=$ 0 , there is no gluonic contribution at all to $g_{1}(x)$ or $g_{2}(x)$ as explained after Eq. (5.1.12), and the constants $a_{n}^{i}, d_{n}^{i}$ of $(5.1 .13,14)$ are then independent of $\mu^{2}$. Now because $\alpha_{s}\left(Q^{2}\right) \rightarrow$ 0 as $Q^{2} \rightarrow \infty$ it often happens that results found in the interacting theory reduce to the structure of the free theory results with just the replacements $a_{n}^{i} \rightarrow a_{n}^{i}\left(Q^{2}\right), d_{n}^{i} \rightarrow d_{n}^{i}\left(Q^{2}\right)$ equivalent, in the Parton Model, to the replacements $q(x) \rightarrow q\left(x, Q^{2}\right)$. If this were always the case then we would expect no gluon contribution to $g_{1}\left(x, Q^{2}\right)$ as $Q^{2} \rightarrow \infty$. But as already mentioned in Section 4, and as will be discussed in much greater detail in Sections 6, 7 and 8 in the framework of the Field Theoretic Parton Model, there is a contribution to $g_{1}\left(x, Q^{2}\right)$ from gluons in the nucleon even in the limit $Q^{2} \rightarrow \infty$. This apparent disagreement between the two approaches has upset many people, leading them to question the validity of the Field Theoretic Parton Model treatment. It must be stressed that there is no disagreement. The limit $g \rightarrow 0$ and the limit $Q^{2} \rightarrow \infty$ are not equivalent. The Operator Product Expansion will give a gluonic contribution to $\Gamma_{1}\left(Q^{2}\right)$ even though no gluonic operator exists for $n=1$. The point is that the gluonic contribution emerges from the quark operators when account is taken of the fact that they are interacting fields and not free fields. There is, in fact, complete equivalence between the approaches as is explained in detail in Section 8.6.

When the detailed results [KOD 79] for the coefficient functions calculated to order $\alpha_{s}$ are fed into (5.2.1) one obtains the fundamental formula (4.4.1) which was the basis for the QCD improved analysis of the polarized DIS data and which leads to the Bjorken sum rule $(4.5 .1,2)$.

\subsection{Is there a connection between $g_{1}(x)$ and $g_{2}(x)$ ?}

There is a much-quoted sum rule, known as the Wandzura-Wilczek relation [WAN 77 which claims to relate $g_{2}(x)$ to an integral over $g_{1}(x)$. The operator product results $(5.2 .1,2)$ allow us to see immediately that the relation cannot be exact.

From (5.2.1) and (5.2.2) we have that

$$
\begin{aligned}
\int_{0}^{1} d x x^{n-1}\left[g_{1}\left(x, Q^{2}\right)+g_{2}\left(x, Q^{2}\right)\right] & =\frac{1}{2 n} \sum_{i} \delta_{i} a_{n}^{i} E_{1, i}^{n}\left(Q^{2}, g\right) \\
& +\frac{n-1}{2 n} \sum_{i} \delta_{i} d_{n}^{i} E_{2, i}^{n}\left(Q^{2}, g\right) \quad(n=3,5,7 \ldots)
\end{aligned}
$$


where, recall, the contribution of the last term on the RHS is of twist 3.

Now, if we assume that (5.3.1) is valid for all integer $n$ - and we shall see in a moment that this may be a dangerous assumption - we can use the convolution theorem for Mellin transforms (which relates the product of the moments of two functions to the moment of their convolution) and Eq. (5.2.1) to obtain

$$
g_{1}\left(x, Q^{2}\right)+g_{2}\left(x, Q^{2}\right)=\int_{x}^{1} \frac{d y}{y} g_{1}\left(y ; Q^{2}\right)+[\text { twist } 3] .
$$

In [WAN 77] it is argued, on the basis of a model, that the twist 3 term can be neglected, leaving a direct connection between $g_{1}$ and $g_{2}$. However, this argument is quite unreliable. The selfsame model gives nonsensical results for $F_{1,2}(x)$ and $g_{1}(x)$ !

Thus, as will be further explained in Section 5.4, the neglect of the twist-3 term in (5.3.2) is dangerous and the Wandzura-Wilczek relation should not be trusted.

But even (5.3.2) may be a dangerous expression. For recall that (5.3.1) does not hold for $n=1$. In extrapolating it to $n=1$ we are tacitly assuming [see (5.2.2)] that the

first moment of $g_{2}, \Gamma_{2}=\int_{0}^{1} d x g_{2}(x)$, vanishes, whereas, as stressed earlier, the Operator Product Expansion gives no direct information on $\Gamma_{2}$ (this assumed vanishing of $\Gamma_{2}$ is known as the Burkhardt-Cottingham sum rule [BUR 70] and will be discussed in the next Section). Thus even (5.3.2) is, a priori, not reliable.

The correct way to proceed is to simply use (5.2.2) with (5.2.1) for $n \geq 3$, i.e.

$$
\int_{0}^{1} d x x^{n-1}\left\{g_{1}(x)+\left(\frac{n}{n-1}\right) g_{2}(x)\right\}=\frac{1}{2} \sum_{i} \delta_{i} d_{n}^{i} E_{2, i}^{n}\left(Q^{2}, g\right) \quad(n \geq 3)
$$

where the RHS is a twist 3 contribution.

This is still a useful equation, for once $g_{1}(x)$ and $g_{2}(x)$ are measured it yields information about the twist 3 operator contribution, which, it turns out, depends upon correlations amongst the partons in the nucleon - a question of great importance because it is just what determines single spin asymmetries in QCD (see Section 10).

\subsection{Does the first moment of $g_{2}(x)$ vanish?}

We have already mentioned that according to the Burkhardt-Cottingham sum rule [BUR 70]

$$
\Gamma_{2}\left(Q^{2}\right)=\int_{0}^{1} d x g_{2}\left(x, Q^{2}\right)=0
$$

We now wish to ask whether or not we can expect this sum rule to hold. Firstly, as stressed in the previous section, the result does not follow from the Operator Product Expansion. In fact the OPE gives no information about the first moment of $g_{2}$ since (5.2.2) is not valid for $n=1$.

The result (5.4.1) was derived originally by considering the asymptotic behaviour of a particular virtual Compton helicity amplitude whose absorptive part is proportional to $g_{2}$, and rests on the assumption that the asymptotic behaviour is controlled by lowlying Regge poles - in this case Regge poles like the $a_{1}$ with $\alpha_{a_{1}}(0) \simeq-0.14$. But a very careful analysis by Heimann [HEI 73] showed that there should be contributions from multi-pomeron and pomeron-Regge cuts thus invalidating the starting assumption in the Burkhardt-Cottingham derivation.

It should be noted that this same argument invalidates the Wandzura-Wilczek result with [twist 3] put equal to zero on the RHS of (5.3.2). For if (5.3.2) were true without the twist 3 term one could deduce (5.4.1) from it directly by integrating over $x$. 
Because of the well known connection between the behaviour of scaling functions in the limit $x \rightarrow 0$ and asymptotic Regge behaviour, the coupling to multi-pomeron cuts implies a highly singular behaviour

$$
g_{2}(x) \stackrel{x \rightarrow 0}{\sim} \frac{1}{x^{2}}
$$

where we have ignored factors of $\ln x$. Thus the integral in (5.4.1) might not even converge!

That said, we should stress that the couplings of Regge poles and cuts is a subtle question and such a remarkable conclusion as (5.4.2) must be tested experimentally. Because Regge cuts always arise from non-planar Feynman diagrams, and since these correspond to twist greater than 2 , it might be that the coefficient of $1 / x^{2}$ in (5.4.2) tends to zero as $Q^{2} \rightarrow \infty$. It is then not clear at what scale of fixed $Q^{2}$ one would expect to observe the divergence experimentally. Alternatively, if $g_{2}(x) \rightarrow C\left(Q^{2}\right) / x^{2}$ as $x \rightarrow 0$ with $C\left(Q^{2}\right) \ll 1$, it is not guaranteed that the divergence will be seen experimentally if $x$ is not small enough.

Heimann has given a clear illustration of the mechanism which could be behind the failure of the Burkhardt-Cottingham sum rule. It involves an illegitimate interchange of double integrals in a Fourier transform.

One can show that $g_{2}(x)$ is given by a Fourier transform of the following type

$$
g_{2}(x)=\int_{-\infty}^{\infty} d \lambda \frac{e^{-i \lambda x}}{2 \pi}[\lambda f(\lambda)]
$$

where the behaviour of $f(\lambda)$ as $\lambda \rightarrow 0$ is such that $\lambda f(\lambda) \rightarrow 0$ as $\lambda \rightarrow 0$.

Now because of its relation to the absorptive part of a scattering amplitude one knows that $g_{2}(x)=0$ for $|x|>1$ and $g_{2}(-x)=g_{2}(x)$. Then

$$
\begin{aligned}
\int_{0}^{1} d x g_{2}(x) \cos \mu x & =\frac{1}{2} \int_{-\infty}^{\infty} d x g_{2}(x) e^{i \mu x} \\
& =\frac{1}{2} \int_{-\infty}^{\infty} d x \int_{-\infty}^{\infty} \frac{d \lambda}{2 \pi} e^{i(\mu-\lambda) x}[\lambda f(\lambda)] .
\end{aligned}
$$

If the integrals can be interchanged, we have

$$
\begin{aligned}
\int_{0}^{1} d x g_{2}(x) \cos \mu x & =\frac{1}{2} \int_{-\infty}^{\infty} d \lambda \delta(\mu-\lambda)[\lambda f(\lambda)] \\
& =\frac{1}{2} \mu f(\mu)
\end{aligned}
$$

so that taking $\mu=0$

$$
\int_{0}^{1} d x g_{2}(x)=0
$$

However it is NOT always legitimate to interchange the integrals, and this is obvious if the integral on the LHS of (5.4.5) diverges, say for $\mu=0$. Indeed the Regge behaviour of $g_{2}(x)$ as $x \rightarrow 0$ is precisely an example of this type.

If one takes

$$
f(\lambda)=\lambda^{\alpha-1} \quad(0<\alpha<1)
$$

so that indeed $\lambda f(\lambda) \rightarrow 0$ as $\lambda \rightarrow 0$, one finds upon taking the Fourier transform (5.4.3), [HEI 73]

$$
g_{2}(x) \propto \frac{1}{x^{1+\alpha}}
$$

and the integral of $g_{2}(x)$ diverges. 


\subsection{The Efremov-Leader-Teryaev sum rule}

If one assumes that (5.3.3) holds also for $n=2$ one obtains

$$
\int_{0}^{1} d x x\left[g_{1}(x)+2 g_{2}(x)\right]=\frac{1}{2} \sum_{i} \delta_{i} d_{2}^{i} E_{2, i}^{2}\left(Q^{2}, g\right) \text {. }
$$

To lowest order in $\alpha_{s}$, because of (5.1.12) we need keep only the operators with $i=1, \ldots, 8$ and $i=\psi$. In that case, from (5.1.14) and (5.1.10 or 11) the $d_{2}$ are of the form

$$
d_{2} \propto\left\langle P, S\left|\overline{\boldsymbol{\psi}} \gamma_{5}[\not(S \cdot D)-\not(n \cdot D)] \boldsymbol{\psi}\right| P, S\right\rangle
$$

where $n^{\mu}$ is a four-vector introduced in the Field Theoretic Parton Model (Section 10) such that $n^{2}=0$ and $n \cdot P=1$.

It can be shown, in the framework of the Field Theoretic Parton Model, that the RHS of (5.5.2) vanishes in the chiral limit. This follows upon integrating eq. (D.20) of Appendix D and using eqs. (D.17, 13 and 6). Hence one has the sum rule [EFR 84]

$$
\int_{0}^{1} d x x\left[g_{1}(x)+2 g_{2}(x)\right]=0
$$

This is further discussed in Appendix E.

It should be noted, however, that like the Burkhardt-Cottingham sum rule, this result does not follow strictly from the OPE. Moreover if $g_{2}(x)$ diverges like, or close to, $1 / x^{2}$ as $x \rightarrow 0$ then, as with the Burkhardt-Cottingham case, the sum rule will fail because of the divergence of the integral of $x g_{2}(x)$.

\section{The axial anomaly and the axial gluon current $\boldsymbol{K}^{\boldsymbol{\mu}}$}

As we have seen in the previous Sections the Naive Parton Model interpretation of the EMC experiment, based upon treating the flavour singlet axial current $J_{5 \mu}^{0}$ as effectively the quark spin-density operator, leads to a negligible contribution to the proton spin from the quark spins, $a_{0}=2 S_{z}^{q u a r k s} \simeq 0$, in contradiction with the Quark Model. The fact that the experiment was done at an average $Q^{2} \simeq 10(\mathrm{GeV} / \mathrm{c})^{2}$ and that the Quark Model is usually trusted for static properties of the nucleon i.e. for small momentum transfers, $Q^{2} \simeq 0$, does not mean that the Naive Parton Model and Quark Model results cannot be compared with each other, as is sometimes claimed, so that there is a real contradiction. The statement that in the Quark Model we deal with constituent quarks and not with partons is somewhat irrelevant, since the states of three constituent quarks can be considered as a sum of states involving three valence partons in the same quantum state as the constituent quarks, with all the other partons (gluons, $q \bar{q}$ pairs etc.) having zero total angular momentum, as emphasized in Lipkin's argument in Section 4.3.2.

Secondly, there appears to be no significant $Q^{2}$-variation in the experimental data down to $Q^{2} \simeq 1(\mathrm{GeV} / \mathrm{c})^{2}$. The sharp change in $\Gamma_{1}^{p}\left(Q^{2}\right)$ demanded by the Drell, Hearn, Gerasimov sum rule occurs for smaller $Q^{2}$ (as discussed in Section 4.1.1), so cannot be used, in the region of the deep inelastic data, as an argument for large higher twist effects.

We must, therefore, return to the foundations of the Parton Model to try to seek a resolution to the problem and we shall learn, surprisingly, of subtle effects which force us to alter some aspects of our naive interpretation of the Parton Model. 


\subsection{Is there really a "spin crisis"?}

In field-theoretical language, the usual statement that the nucleon predominantly consists of three quarks in an $S U(6)$ state (with zero orbital angular momentum) means that the 3 quarks $\rightarrow 3$ quarks Green function with these quantum numbers has a pole at the mass of the nucleon, with $J=1 / 2$, with a significant residue.

The fact that the nucleon is not just a 3 quark state means that the nucleon pole also occurs, albeit with smaller residue, in more complicated Green functions such as $3 q+g \rightarrow 3 q+g$, perhaps with non-zero orbital angular momentum. The operator $J_{5 \mu}^{0}$, containing quark field operators only, is the quark spin density operator for free fields and therefore it does measure the total quark spin in the initial parton state. But since, as we shall see, $J_{5 \mu}^{0}$ is not a conserved current the expectation value in the nucleon state where the partons interact strongly with each other is not the same as the expectation value in the initial state made up of free partons. Only in the Naive Parton Model, where we ignore these interactions, can we equate the expectation values in the nucleon and free parton states. Since everybody knows that only total $J_{z}$ and not $S_{z}$ is generally conserved one may wonder why it is even necessary to emphasize the non-conservation of $J_{5 \mu}^{0}$. The reason is that naively, i.e. from the equations of motion, $J_{5 \mu}^{0}$ is conserved for massless quarks. So the non-conservation is anomalous if one works with massless quarks as is often done in the Parton Model.

For $J_{5 \mu}^{3}$ and $J_{5 \mu}^{8}$ the conservation is exact with massless quarks. It is also exact if $S U(2)$ and $S U(3)$ are exact symmetries. Since these symmetries are slightly broken the conservation will be slightly imperfect with quarks of non-zero mass. The currents are then partially conserved. We take it that any dependence on the renormalization scale for $J_{5 \mu}^{3}$ and $J_{5 \mu}^{8}$ can be neglected.

But there is a further consequence of the non-conservation of $J_{5 \mu}^{0}$ which puts into question our interpretation of the expectation value of $J_{5 \mu}^{0}$ as the mean value of the total parton spin. To see this consider the protonic matrix element $\left\langle P\left|J_{5 \mu}^{0}\right| P\right\rangle$ in the Heisenberg picture. We may insert a sum over free parton states (assuming, as usual, completeness) so that

$$
\left\langle P\left|J_{5 \mu}^{0}\right| P\right\rangle=\sum\left|\left\langle k_{1} \ldots k_{n} \mid P\right\rangle\right|^{2}\left\langle k_{1} \ldots k_{n}\left|J_{5 \mu}^{0}\right| k_{1} \ldots k_{n}\right\rangle .
$$

Strictly speaking the parton states should either be 'IN' or 'OUT' type states, but this is irrelevant for our discussion.

Now in an interacting quantum field theory we are forced to renormalize the fields and operators, with the result that the value of matrix elements like $\left\langle k_{1} \ldots k_{n}|\hat{O}| k_{1} \ldots k_{n}\right\rangle$ will, in general, depend on the renormalization scale $\mu^{2}$. Only if the operator $\hat{O}$ is conserved can one show that the matrix elements are independent of $\mu^{2}$. This is the case for $J_{5 \mu}^{3}$ and $J_{5 \mu}^{8}$ with massless quarks. Since anything of real physical content must be independent of $\mu^{2}$ we see that the expectation value of non-conserved operator cannot have any simple physical significance. Thus it is misleading to think of the expectation value of $J_{5 \mu}^{0}$ as "the physical spin of the parton" - it is not a fixed number, it depends on the value of $\mu^{2}$ and it can, in principle, have any value whatsoever. To emphasize this one should always indicate the renormalization scale $i . e$. write $\left\langle k_{1} \ldots k_{n}\left|J_{5 \mu}^{0}\right| k_{1} \ldots k_{n}\right\rangle_{\mu^{2}}$. (Sometimes one refers to this as the spin at scale $\mu^{2}$, but there is no implication that its value should be of order 1.)

Bearing this in mind we see that Lipkin's argument (Section 4.3.2) loses its force in the context of an interacting quantum field theory. The spin crisis is a crisis only in the domain of the Naive Parton Model, as was indeed claimed in the paper which introduced 
the concept of a "crisis" [LEA 88].

\subsection{On the connection between QCD, the Quark Model and the Naive Parton Model}

Given that the expectation value of $J_{5 \mu}^{0}$ depends upon the renormalization scale $\mu^{2}$ we would like to know if there is a value of $\mu^{2}$ at which the expectation value agrees with the Quark Model result i.e. corresponds to the physical spin of free quarks. We shall suggest that this happens as $\mu^{2} \rightarrow 0$, but because the Quark Model is an effective theory of the non-perturbative regime of QCD we do not have a rigorous argument. However we shall adduce some arguments from the perturbative domain to support our contention.

The Quark Model is characterized by the fact that it does not contain gluons; the dynamics is in the quark degrees of freedom. Now neither the quark momentum operator nor the gluon momentum operator is conserved in QCD. Only their sum is. Thus the momentum fractions of a hadron carried by quarks or by gluons each depend upon the renormalization scale. For large $\mu^{2}$, where $\alpha_{s}\left(\mu^{2}\right)$ is small so that perturbation theory can be trusted, we know that the momentum fraction carried by the gluons increases to the limit $16 / 25$ at $\mu^{2} \rightarrow \infty$. Thus the gluons play a smaller rôle in the momentum sum rule at smaller $\mu^{2}$. Similarly, we shall see in Section 8 that the spin at scale $\mu$ carried by the gluons increases without limit as $\mu^{2} \rightarrow \infty$, so that gluons play a lesser rôle in the angular momentum sum rule at lower $\mu^{2}$.

Both these examples suggest that one is approaching the Quark Model as $\mu^{2} \rightarrow 0$. Of course the above are perturbative arguments but it seems reasonable to assume that the trend continues down to non-perturbatively small values of $\mu^{2}$. At the other end of the scale we have the partonic picture which was invented to explain Bjorken scaling which holds as $Q^{2} \rightarrow \infty$, which in the present context corresponds to $\mu^{2} \rightarrow \infty$. And indeed, since $\alpha_{s}\left(\mu^{2}\right) \rightarrow 0$ as $\mu^{2} \rightarrow \infty$ one does usually obtain the relationships of the Naive Parton Model in this limit, but in fact one never recovers Bjorken scaling and one has to utilize $Q^{2}$-dependent parton distributions.

The key exception to this rule is the gluonic contribution to the first moment of $g_{1}\left(x, Q^{2}\right)$. Here, as will be seen in Section 6.3 and 7.2, we have a QCD correction proportional to $\alpha_{s}\left(Q^{2}\right)$ multiplied by the gluon spin at scale $Q^{2}$, which increases like $\ln Q^{2}$. The logarithmic decrease of $\alpha_{s}\left(Q^{2}\right)$ as $Q^{2} \rightarrow \infty$ is just compensated by the increase in the gluon spin, leaving a finite, non-zero contribution as $Q^{2} \rightarrow \infty$. This is quite anomalous and is, indeed, linked directly to the existence of the axial anomaly in QCD. It is thus a counterexample to the usual rule that one recovers the relationships of the Naive Parton Model in the limit $Q^{2} \rightarrow \infty$.

This discovery catalyzed by the EMC result is of profound importance and, ironically, will stand, even if it turns out that the EMC result is modified by future experiments.

\subsection{The axial anomaly}

Consider the axial current

$$
J_{5 \mu}^{f}=\bar{\psi}_{f}(x) \gamma_{\mu} \gamma_{5} \psi_{f}(x)
$$

made up of quark operators of definite flavour $f$. (An implicit colour sum is always implied). From the free Dirac equation of motion one finds that

$$
\partial^{\mu} J_{5 \mu}^{f}=2 i m_{q} \bar{\psi}_{f}(x) \gamma_{5} \psi_{f}(x)
$$


where $m_{q}$ is the mass of the quark of flavour $f$.

In the chiral limit $m_{q} \rightarrow 0$ (6.3.2) appears to imply that $J_{5 \mu}^{f}$ is conserved. If this were really true there would be a symmetry between left and right-handed quarks, leading to a parity degeneracy of the hadron spectrum e.g. there would exist two protons, of opposite parity. However, the formal argument from the free equations of motion is not reliable and, as shown originally by Adler, and by Bell and Jackiw [ABJ 69] (in the context of QED), there is an anomalous contribution arising from the triangle diagram shown in Fig. 6.1. As a consequence the axial current is not conserved when $m_{q}=0$. One has instead, for the QCD case

$$
\partial^{\mu} J_{5 \mu}^{f}=\frac{\alpha_{s}}{4 \pi} G_{\mu \nu}^{a} \widetilde{G}_{a}^{\mu \nu}=\frac{\alpha_{s}}{2 \pi} \operatorname{Tr}\left[\boldsymbol{G}_{\mu \nu} \widetilde{\boldsymbol{G}}^{\mu \nu}\right]
$$

where $\widetilde{G}_{\mu \nu}^{a}$ is the dual field tensor

$$
\widetilde{G}_{\mu \nu}^{a} \equiv \frac{1}{2} \varepsilon_{\mu \nu \rho \sigma} G_{a}^{\rho \sigma}
$$

and where a field vector or tensor without a colour label stands for a matrix. In this case

$$
\boldsymbol{G}_{\mu \nu} \equiv\left(\frac{\boldsymbol{\lambda}_{a}}{2}\right) G_{\mu \nu}^{a} .
$$

The result (6.3.3), which emerges from a calculation of the triangle diagram (Fig. 6.1) using $m_{q}=0$ and the gluon virtuality $k^{2} \neq 0$, is really a particular limit of a highly non-uniform function. If we take $m_{q} \neq 0, k^{2} \neq 0$ the RHS of (6.3.3) is multiplied by

$$
T\left(m_{q}^{2} / k^{2}\right)=1-\frac{2 m_{q}^{2} / k^{2}}{\sqrt{1+4 m_{q}^{2} / k^{2}}} \ln \left(\frac{\sqrt{1+4 m_{q}^{2} / k^{2}}+1}{\sqrt{1+4 m_{q}^{2} / k^{2}}-1}\right) .
$$

We see that this anomaly corresponds to $T \rightarrow 1$ for $\left(m_{q}^{2} / k^{2}\right) \rightarrow 0$. On the other hand, for on-shell gluons, $k^{2}=0$, and $m_{q} \neq 0$, i.e. in the limit $\left(m_{q}^{2} / k^{2}\right) \rightarrow \infty$ the terms cancel, $T \rightarrow 0$, and there is no anomaly. As explained in Section 8.1 the particular case $k^{2}=0$ is irrelevant and there is no doubt that the anomaly is relevant.

The anomaly induces a pointlike interaction between $J_{5 \mu}^{0}$ and gluons. That it is pointlike can be seen by taking different gluon momenta $k_{1}$ and $k_{2}$ in Fig. 6.1 and noting that the amplitude does not depend on the momentum transfer $k_{1}-k_{2}$ when $m_{q}=$ 0 . Therefore, in computing the matrix element of $J_{5 \mu}^{0}$ in a hadron state, we will get a contribution from the gluon components of the hadron as well as the more obvious contribution from quarks. From Adler's expression [ABJ 69] for the triangle diagram, modified to QCD, one finds for the forward gluonic matrix element of the flavour $f$ current (our convention is $\varepsilon_{0123}=1$ )

$$
\begin{aligned}
\left\langle k, \lambda\left|J_{5 \mu}^{f}\right| k, \lambda\right\rangle & =\frac{i \alpha_{s}}{2 \pi} \varepsilon_{\mu \nu \rho \sigma} k^{\nu} \varepsilon^{* \rho}(\lambda) \varepsilon^{\sigma}(\lambda) T\left(m_{q}^{2} / k^{2}\right) \\
& =-\frac{\alpha_{s}}{2 \pi} S_{\mu}^{g}(k, \lambda) T\left(m_{q}^{2} / k^{2}\right)
\end{aligned}
$$

where $\lambda$ is the gluon helicity and we may take

$$
S_{\mu}^{g}(k, \lambda) \approx \lambda k_{\mu}
$$

as the covariant spin vector for almost massless gluons. 
Using the methods of Section 3 we may then compute the gluonic contributions to the hadronic expectation value $\left\langle P, S\left|J_{5 \mu}^{0}\right| P, S\right\rangle$. In this case the gluons being bound will be slightly off-shell i.e. $k^{2} \neq 0$, but small. The full triangle contribution involves a sum over all quark flavours. We take $m_{u}, m_{d}$ and $m_{s}$ to be $\ll k^{2}$ whereas $m_{c}, m_{b}$ and $m_{t}$ are $\gg k^{2}$. The function $T\left(m_{q}^{2} / k^{2}\right)$ thus takes the values:

$$
\begin{array}{llll}
T=1 & \text { for } & u, d, s \\
T=0 & \text { for } & c, b, t
\end{array}
$$

and the gluon contribution is then given by [see (4.2.8)]

$$
\begin{aligned}
a_{0}^{\text {gluons }}\left(Q^{2}\right) & =-3 \frac{\alpha_{s}}{2 \pi} \int_{0}^{1} d x \Delta g\left(x, Q^{2}\right) \\
& =-3 \frac{\alpha_{s}}{2 \pi} \Delta g\left(Q^{2}\right)
\end{aligned}
$$

or from $(4.2 .1)$

$$
\Gamma_{1 p}^{\text {gluons }}\left(Q^{2}\right)=-\frac{1}{3} \frac{\alpha_{s}}{2 \pi} \Delta g\left(Q^{2}\right) .
$$

$\Delta g\left(x, Q^{2}\right)$ is the difference between the number density of gluons with the same helicity as the nucleon and those with opposite helicity; its integral $\Delta g\left(Q^{2}\right)$ is the total helicity carried by the gluons. Note that if $N_{f}$ massless quark flavours contribute in the anomalous triangle then on the RHS of (6.3.11)

$$
1 / 3 \rightarrow N_{f}\left\langle e_{f}^{2}\right\rangle / 2
$$

where $\left\langle e_{f}^{2}\right\rangle$ is the mean of the squared charges.

Although (6.3.3) was derived perturbatively to order $\alpha_{s}$, it is believed to be an exact result. It was shown long ago by Adler [ABJ 69] that the anomaly is not influenced by higher order corrections at the 2-loop level. More recently Anselm and Johansen [ANS 89a] showed that the 3-loop diagram of Fig. 6.2 effectively multiplies the anomaly result by a cut-off dependent constant. But this constant is the same as the one shown by Adler to renormalize $J_{5 \mu}^{0}$ via Fig. 6.3. Consequently the QED version of (6.3.3) is unchanged. These results remain true in QCD for the matrix elements of (6.3.3). Further, it has been argued by Jackiw [JAC 85] that (6.3.3) is true even outside the perturbative realm of QCD.

If we consider the anomaly contribution to the hadronic matrix element then it might appear possible to regard the contribution (6.3.11) as arising from a QCD correction to the quark distribution function. However this is quite incorrect since the important region of $p^{2}$ for the quark lines in the triangle loop integral, must be $p^{2} \rightarrow \infty$ in order to produce the pointlike behavior. The triangle really should be regarded as a point interaction between the current and the gluons. The result (6.3.10) is of fundamental importance. It tells us that the Naive Parton Model formula (4.3.4) for $a_{0}$ (and hence for $\Gamma_{1}^{p}$ in terms of the $\left.\Delta q_{f}\right)$ is incorrect. We now have, instead,

$$
a_{0}=\Delta \Sigma-3 \frac{\alpha_{s}}{2 \pi} \Delta g
$$

This result will be discussed in some detail in Section 7. But we note, immediately, that it has the fundamental implication that the small measured value of $a_{0}$ does not necessarily imply that $\Delta \Sigma$ is small. 


\subsection{The axial gluon current $K^{\mu}$ and the gluon spin}

It is not difficult to show that if one introduces an axial gluon current

$$
\begin{aligned}
K^{\mu} & =\frac{1}{2} \varepsilon^{\mu \nu \rho \sigma} A_{\nu}^{a}\left(G_{\rho \sigma}^{a}-\frac{g}{3} f_{a b c} A_{\rho}^{b} A_{\sigma}^{c}\right) \\
& =\varepsilon^{\mu \nu \rho \sigma} \operatorname{Tr}\left\{\boldsymbol{A}_{\nu}\left(\boldsymbol{G}_{\rho \sigma}+\frac{i}{3} g\left[\boldsymbol{A}_{\rho}, \boldsymbol{A}_{\sigma}\right]\right)\right\}
\end{aligned}
$$

where, as earlier, the matrix $\boldsymbol{A}_{\rho}=\frac{\boldsymbol{\lambda}_{a}}{2} A_{\rho}^{a}$, then

$$
\partial_{\mu} K^{\mu}=\frac{1}{2} G_{\mu \nu}^{a} \widetilde{G}_{a}^{\mu \nu}=\operatorname{Tr}\left(\boldsymbol{G}_{\mu \nu} \widetilde{\boldsymbol{G}}^{\mu \nu}\right) .
$$

Consequently, if $m_{q}=0$, the modified current

$$
\widetilde{J}_{5 \mu}^{f} \equiv J_{5 \mu}^{f}-\frac{\alpha_{s}}{2 \pi} K_{\mu}
$$

is conserved, $\partial^{\mu} \widetilde{J}_{5 \mu}^{f}=0$.

The matrix elements of the modified singlet axial current

$$
\widetilde{J}_{5 \mu}^{0} \equiv J_{5 \mu}^{0}-N_{f} \frac{\alpha_{s}}{2 \pi} K_{\mu}
$$

are independent of the renormalization scale and should correspond with the value obtained in the Quark Model (no gluons; approximately $S U(6)$ quark wave function) i.e. in the analogue of (4.2.8),

$$
\left\langle P, S\left|\widetilde{J}_{5 \mu}^{0}\right| P, S\right\rangle=2 M \tilde{a}_{0} S^{\mu},
$$

we expect $\tilde{a}_{0}$ independent of $Q^{2}$ and thus $\tilde{a}_{0} \simeq 1$.

We remarked in Section 1 that many of the operators corresponding to standard dynamical observables are in fact non gauge-invariant in a local gauge theory. In the gauge $A_{0}^{a}(x)=0$ the gluon spin operator $\hat{\boldsymbol{S}}^{g}$ becomes

$$
\hat{S}_{i}^{g}=-\varepsilon_{i j k} A_{a}^{j} \partial^{0} A_{a}^{k} .
$$

But in this gauge the cubic term vanishes for the spatial components of the vector $K^{\mu}$ and one finds

$$
K_{i}=-\hat{S}_{i}^{g} \quad\left(\text { gauge } A_{a}^{0}=0\right) .
$$

Consider now the hadronic expectation value of $K^{\mu}$. By the methods of Section 3, and in accord with (6.4.7) we find, in the gauge $A_{0}=0$,

$$
\left\langle P, S\left|K^{\mu}\right| P, S\right\rangle=-2 M S^{\mu}(P) \Delta g .
$$

Let us now consider the question of the gauge dependence of this relation. The current $K^{\mu}$ is not gauge invariant. In an Abelian theory like QED there is no cubic term and the gauge transformation induced in $K^{\mu}$ as a consequence of

$$
A_{\mu}(x) \rightarrow A_{\mu}(x)+\partial_{\mu} \Lambda(x)
$$

can be written in the form

$$
K_{\mu}(x) \rightarrow K_{\mu}(x)-\frac{1}{2}\left[\partial^{\nu} \Lambda(x)\right] \varepsilon_{\mu \nu \rho \sigma} F^{\rho \sigma}(x)
$$


where we have used the fact that $F^{\mu \nu}$ is gauge invariant in QED. Although $K_{\mu}(x)$ changes, its forward matrix elements, or expectation values do not, since the expectation value of $F^{\rho \sigma}(x)$ vanishes. The latter follows because in QED $F^{\rho \sigma}$ is related to the $A_{\mu}(x)$ entirely via derivatives, and one may utilize $\left[\hat{P}_{\mu}, f(x)\right]=-i \partial f / \partial x^{\mu}$.

In QCD, under

$$
\boldsymbol{A}_{\mu} \rightarrow \boldsymbol{U} \boldsymbol{A}_{\mu} \boldsymbol{U}^{-1}+\frac{i}{g}\left(\partial_{\mu} \boldsymbol{U}\right) \boldsymbol{U}^{-1}
$$

one has

$$
\boldsymbol{G}_{\mu \nu} \rightarrow \boldsymbol{U} \boldsymbol{G}_{\mu \nu} \boldsymbol{U}^{-1}
$$

and one ends up with

$$
\begin{aligned}
K_{\mu} & \rightarrow K_{\mu}+\frac{2 i}{g} \varepsilon_{\mu \nu \alpha \beta} \partial^{\nu} \operatorname{Tr}\left(\boldsymbol{A}^{\alpha} \boldsymbol{U}^{-1} \partial^{\beta} \boldsymbol{U}\right) \\
& +\frac{2}{3 g^{2}} \varepsilon_{\mu \nu \alpha \beta} \operatorname{Tr}\left\{\boldsymbol{U}^{-1}\left(\partial^{\nu} \boldsymbol{U}\right) \boldsymbol{U}^{-1}\left(\partial^{\alpha} \boldsymbol{U}\right) \boldsymbol{U}^{-1}\left(\partial^{\beta} \boldsymbol{U}\right)\right\}
\end{aligned}
$$

The second term in the RHS of (6.4.13) is a total divergence and gives zero contribution to the expectation value of $K_{\mu}$. The third term can also be shown to be a divergence [CRO 83] but in this case cannot be discarded because of the non-trivial topological structure of QCD as discussed in Section 8.1. The last term may indeed be ignored for "small" gauge transformations i.e. those continuously connected to the unit transformation $\boldsymbol{U}=\boldsymbol{I}$, but it cannot be ignored for "large" (topologically non-trivial) gauge transformations. We shall return to study the consequence of this in Sections 8.1 and 8.4.

\section{$7 \quad$ Reinterpretation of the measurement of $\Gamma_{1}^{p}$}

\subsection{The rôle of the anomalous gluon contribution}

We showed in Section 4, that the measurement of $g_{1}^{p}$ can be interpreted as effectively the measurement of $a_{0}$ which is proportional to the proton expectation value of the flavour singlet axial vector current $J_{5 \mu}^{0}$. We also argued that the value found by the EMC i.e. $a_{0} \simeq 0$ was most unintuitive in the framework of the Naive Parton Model, since, therein, it implies $\Delta \Sigma \simeq 0$.

On account of the anomaly, the connection between $a_{0}$ and $\Delta \Sigma$ is quite different from what it is in the Naive Parton Model, and we now have [see (6.3.12)]

$$
a_{0}\left(Q^{2}\right)=\Delta \Sigma-3 \frac{\alpha_{s}\left(Q^{2}\right)}{2 \pi} \Delta g\left(Q^{2}\right)
$$

instead of (4.3.4).

The anomaly has generated an effective point interaction between the virtual photon and the gluons and the small value of $a_{0}$ can now arise as a result of a cancellation between $\Delta \Sigma$ and the $Q^{2}$-dependent gluon spin contribution $\left(3 \alpha_{s}\left(Q^{2}\right) / 2 \pi\right) \Delta g\left(Q^{2}\right)$. Why these terms should roughly cancel will be discusses presently.

Quantitatively if we take $a_{0} \simeq 0.17$ at $Q^{2}=10(\mathrm{GeV} / \mathrm{c})^{2}$ where $\alpha_{s} \simeq 0.24$ we can let the quarks carry, say $60 \%$ of the proton spin i.e. choose $\Delta \Sigma=0.6$ and (7.1.1) then implies

$$
\Delta g\left[Q^{2}=10(\mathrm{GeV} / \mathrm{c})^{2}\right] \simeq 3.8
$$


This is, at first sight, quite unphysically large Đ. But it should not be forgotten that according to the discussion in Section 6.1, this is the spin carried by the gluons at scale $10(\mathrm{GeV} / \mathrm{c})^{2}$ and its value could be anything. To get some idea as to whether this value is absurd or not, we can use the evolution equation to be derived in Section 7.2 to estimate $\Delta g\left(Q^{2}\right)$ at some lower scale, closer to the Quark Model regime. Going as far as we dare, we take $Q^{2}=4 \Lambda_{\mathrm{QCD}}^{2}$ where $\alpha_{s} \simeq 1$ and find that

$$
\Delta g\left(4 \Lambda_{\mathrm{QCD}}^{2}\right) \simeq 0.7,
$$

a not unreasonable value.

In (7.1.1) we have shown no $Q^{2}$-dependence in $\Delta \Sigma$. This follows upon using (6.4.3, 5 and 8) to get

$$
\begin{aligned}
\tilde{a}_{0} & =\left(\Delta \Sigma-3 \frac{\alpha_{s}}{2 \pi} \Delta g\right)+3 \frac{\alpha_{s}}{2 \pi} \Delta g \\
& =\Delta \Sigma
\end{aligned}
$$

and the fact that $\widetilde{a}_{0}$, being related to the conserved current $\widetilde{J}_{5 \mu}^{0}$ is independent of the renormalization scale $\mu^{2}$ (or $Q^{2}$ ), as we shall explain below.

Consider the axial charge $\widetilde{Q}_{5}$ associated with the conserved current $\widetilde{J}_{5 \mu}^{0}$. We have

$$
\widetilde{Q}_{5}=\int d^{3} \boldsymbol{x} \widetilde{J}_{50}^{0}(\boldsymbol{x}, t)
$$

Let us ask how it would be possible for $\widetilde{Q}_{5}$ to depend upon the renormalization scale $\mu^{2}$, in a massless theory. The only way would be via the variable $\mu t$ and such a dependence would then induce a dependence upon $t$. But we know that for a conserved local current the charge is time-independent. Thus $\widetilde{Q}_{5}$ must be independent of the renormalization scale. It follows, as claimed after Eq. (7.1.4), that $\widetilde{a}_{0}$ or $\Delta \Sigma$ are independent of $Q^{2}$.

\subsection{Why the gluon contribution survives as $Q^{2} \rightarrow \infty$}

At first sight it seems surprising that the gluonic term in (7.1.1) survives at large $Q^{2}$. It looks like an $\alpha_{s}$ correction which usually is expected to disappear as $Q^{2} \rightarrow \infty$. However, this is misleading since the gluon spin at scale $Q^{2}$ behaves just like $\left[\alpha_{s}\left(Q^{2}\right)\right]^{-1}$ as $Q^{2} \rightarrow \infty$. This can be seen either from the spin-dependent Altarelli-Parisi evolution equations [ALT 77] or from the anomalous dimensions of the operators involved. We shall outline the latter approach.

Now the axial current $J_{5 \mu}^{0}$ is multiplicatively renormalized which implies that the dependence on renormalization scale appears in a multiplicative factor. Consequently one can write

$$
\frac{d}{d \ln Q^{2}}\left[\Delta \Sigma-\frac{3 \alpha_{s}\left(Q^{2}\right)}{2 \pi} \Delta g\left(Q^{2}\right)\right]=-\gamma\left(\alpha_{s}\right)\left[\Delta \Sigma-\frac{3 \alpha_{s}\left(Q^{2}\right)}{2 \pi} \Delta g\left(Q^{2}\right)\right]
$$

where $\gamma\left(\alpha_{s}\right)$ is the anomalous dimension of $J_{\mu 5}^{0}$.

This is the standard form of an anomalous dimension evolution equation. What is a little unusual is that the power series expansion for $\gamma\left(\alpha_{s}\right)$ begins only in second order [KOD 80] i.e.

$$
\gamma\left(\alpha_{s}\right)=\gamma_{2}\left(\frac{\alpha_{s}}{4 \pi}\right)^{2}+\ldots
$$

1) Note added in proof: A value of $a_{0} \simeq 0.27$ (see footnote in Section 4.4.1, [ADA 94]) would imply a smaller value $\Delta g\left[Q^{2}=10(\mathrm{GeV} / \mathrm{c})^{2}\right] \simeq 3.0$. 
with

$$
\gamma_{2}=16 N_{f}
$$

The solution to (7.2.1) is then

$$
\left[\Delta \Sigma-\frac{3 \alpha_{s}}{2 \pi} \Delta g\right]_{Q^{2}}=\left[\Delta \Sigma-\frac{3 \alpha_{s}}{2 \pi} \Delta g\right]_{Q_{0}^{2}} \times \exp \left\{\frac{\gamma_{2}}{4 \pi \beta_{0}}\left[\alpha_{s}\left(Q^{2}\right)-\alpha_{s}\left(Q_{0}^{2}\right)\right]\right\}
$$

where, as usual,

$$
\beta_{0}=11-\frac{2}{3} N_{f}
$$

Equation (7.2.4) implies that the quantity

$$
\left[\Delta \Sigma-3 \frac{\alpha_{s}\left(Q^{2}\right)}{2 \pi} \Delta g\left(Q^{2}\right)\right] \times \exp \left\{-\frac{\gamma_{2}}{4 \pi \beta_{0}} \alpha_{s}\left(Q^{2}\right)\right\}
$$

is independent of $Q^{2}$. Let us put it equal to a constant $C$, where $C$ is the value of (7.2.6) at some arbitrary chosen value of $Q^{2}$.

We now take the limit $Q^{2} \rightarrow \infty$. Since $\Delta \Sigma$ is independent of $Q^{2}$ and $\alpha_{s}\left(Q^{2}\right) \rightarrow 0$ we obtain

$$
\lim _{Q^{2} \rightarrow \infty} \frac{3 \alpha_{s}\left(Q^{2}\right)}{2 \pi} \Delta g\left(Q^{2}\right)=\Delta \Sigma-C
$$

Thus it must be that $\Delta g\left(Q^{2}\right)$ increases like $\left[\alpha_{s}\left(Q^{2}\right)\right]^{-1}$ as $Q^{2}$ increases.

\subsection{The angular momentum sum rule revisited}

We have suggested that the results on the first moment in polarized deep inelastic lepton-hadron scattering can be explained as a cancellation between a reasonably large quark spin contribution $\Delta \Sigma \simeq(0.6-0.7)$, as expected intuitively, and the anomalous gluon contribution $\Delta g$. The above value of $\Delta \Sigma$ is consistent with various relativistic bag model calculations and, as will be explained in Section 9.3, with the so-called "Generalized Goldberger-Treiman relation".

However, in order to accomplish this cancellation, one requires a large gluon spin $\Delta g$ at $\left\langle Q^{2}\right\rangle \simeq 10(\mathrm{GeV} / \mathrm{c})^{2}$ i.e. $\Delta g \simeq 4$. As we have explained $\Delta g$ grows indefinitely as $Q^{2}$ increases according to the usual QCD evolution equations so that such a large value of $\Delta g$ cannot be ruled out by our expectation that the gluon contribution is small at very small $Q^{2}$.

It should be noted that the large $\Delta g$ has important consequences for the total angular momentum sum rule. For a nucleon with helicity $+1 / 2$ moving along the $z$-axis we must have

$$
J_{z}=S_{z}^{q}+S_{z}^{g}+L_{z}=\frac{1}{2}
$$

where $L_{z}$ represents the total orbital angular momentum of all partons. With $S_{z}^{q} \simeq 0.7$ and independent of $Q^{2}$, the growing value of $S_{z}^{g}$ has to be compensated by an analogous growth in the magnitude of $L_{z}$. A detailed analysis of how this happens has been given by Ratcliffe [RAT 87].

In the process of evolution of a quark it radiates a gluon in some preferred helicity state and, in so doing, conserves its own helicity. It can then radiate again, with the gluon again produced in the same preferred helicity state. Thus gluons of some definite helicity are preferentially being radiated and $\Delta g$ thus increases. But since the total angular 
momentum is conserved at each step of the evolution, something has to compensate for the component of spin carried by the radiated gluon. This, it turns out, is provided by the orbital angular momentum of the $q g$-pair in the process $q \rightarrow q g$. Hence as $\Delta g$ increases with each step in the evolution, so too does $L_{z}$ with opposite sign.

The angular momentum sum rule (7.3.1) is thus completely in accord with the QCD growth of both $S_{z}^{g}$ and $L_{z}$ as $Q^{2}$ increases.

\section{The anomaly in the QCD Field Theoretic Model}

We would like to try to understand the previous results concerning the rôle of the anomaly in $\Gamma_{1}^{p}$ in more intuitive terms, in particular in the framework of the Feynman diagram approach which provides a more rigorous, field-theoretic approach than the Parton Model [EFR 88, ALT 88, LEA 88a, CAR 88]. (We refer to this picture as the QCD Field Theoretic Model). We also wish to study the gluon contribution to $g_{1}(x)$ itself.

\subsection{The factorization theorem}

According to the factorization theorem, which is the basis for deriving Parton Model type results from field-theory, and which has been proved to all orders in perturbative QCD, the cross-section asymmetry $\Delta \sigma_{\gamma^{*} p}$

$$
\Delta \sigma_{\gamma^{*} p} \equiv \sigma_{1,1 / 2}-\sigma_{-1,1 / 2}
$$

for the absorption of a virtual photon on a proton of momentum $P$ (the subscripts label helicities), is given by

$$
\begin{aligned}
\Delta \sigma_{\gamma^{*} p} & =\int_{0}^{1} d x^{\prime}\left\{\sum_{f}\left[\Delta q_{f}\left(x^{\prime}\right)+\Delta \bar{q}_{f}\left(x^{\prime}\right)\right] \Delta \sigma_{\gamma^{*} q}\left(x^{\prime} P\right)\right. \\
& \left.+\Delta g\left(x^{\prime}\right) \Delta \sigma_{\gamma^{*} g}\left(x^{\prime} P\right)\right\}
\end{aligned}
$$

where the sum is over flavours and the partonic cross-sections refer to photo-absorption on quarks or gluons of momentum $p=x^{\prime} P$. In the following we consider the $\gamma^{*}$-proton collision either in the Lab frame with $\gamma^{*}$ moving along $O Z$ or in an 'infinite momentum' frame where the fast proton and $\gamma^{*}$ move parallel to $O Z$.

The partonic sub-process $\gamma^{*} q$ is dominated by the Born diagram in Fig. 8.1 which physically corresponds to a forward jet with essentially zero $k_{T}$ (momentum perpendicular to the photon direction) in the Lab frame or in an 'infinite momentum frame' boosted from the Lab frame along the photon direction. (Of course there is another jet, in the backward direction in the frame where the $\gamma^{*}$ energy is zero, arising from the fragments of the proton). Integrated over $k_{T}$ the Born contribution leads to the usual expression for $g_{1}^{p}$ that one obtains in the Naive Parton Model.

The partonic sub-process $\gamma^{*} g$ is dominated by $q \bar{q}$ pair production as shown in Fig. 8.2. It is characterized by the formation of two jets with essentially opposite $\boldsymbol{k}_{T}$ and is distinguishable from the process of Fig. 8.1 provided $k_{T}$ is large enough. If one calculates the differential cross-section asymmetry $d \Delta \sigma_{\gamma^{*} g} / d k_{T}^{2}$, where $\boldsymbol{k}_{T}$ and $-\boldsymbol{k}_{T}$ are the jet momenta perpendicular to $O Z$, one finds an interesting dependence on the quark mass, as shown in Fig. 8.3, where the $k_{T^{-}}$-dependence is shown for three choices of quark mass: $m_{q}^{2} \simeq Q^{2}, m_{q}^{2} \neq 0$ but $\ll Q^{2}$ and $m_{q}^{2}=0$. For $k_{T} \gg m_{q}$ it is negative and for $k_{T} \ll m_{q}$ it is positive. For $m_{q}=0$ it is thus always negative. Integrated over $k_{T}$ one 
gets for the contribution to the scaling function of the partonic process $\ell g \rightarrow \ell X, g_{1}^{\gamma^{*} g}(x)$, from Fig. 8.2, for each quark flavour $f$ [BAS 93]

$$
\begin{aligned}
g_{1}^{\gamma^{*} g}\left(x, Q^{2}\right) & =\frac{e_{f}^{2}}{2} \frac{\alpha_{s}}{2 \pi}\left\{(2 x-1)\left[\ln \left(\frac{Q^{2}(1-x) / x}{x(1-x) p^{2}+m_{q}^{2}}\right)-1\right]\right. \\
& \left.+(1-x) \frac{2 m_{q}^{2}-x(2 x-1) p^{2}}{x(1-x) p^{2}+m_{q}^{2}}\right\} .
\end{aligned}
$$

Here $x$ stands for the Bjorken variable of the partonic process, $x=Q^{2} / 2 p \cdot q$. For $m_{q}^{2}=0$ this simplifies to

$$
\left.g_{1}^{\gamma^{*} g}\left(x, Q^{2}\right)\right|_{m_{q}^{2}=0}=\frac{e_{f}^{2}}{2} \frac{\alpha_{s}}{2 \pi}(2 x-1)\left[\ln \left(Q^{2} / p^{2}\right)-2(\ln x+1)\right] .
$$

We now wish to feed this into the analogue of (8.1.2) in order to obtain the gluonic contribution to $g_{1}^{p}(x)$. In doing so we must remember that in the QCD Field Theoretic Model the convolution formula (8.1.2) emerges as the lowest twist approximation to what was originally a Feynman diagram with an integration $\int d^{4} p$. In this integration the point $p^{2}=0$ is of zero measure. We saw in Section 6.3 that the anomaly is present if $p^{2} \neq 0$, but absent if $p^{2}=0$. From the present point of view the case $p^{2}=0$ is irrelevant and it is quite clear that the anomaly does contribute to DIS.

It should also be remembered that in the QCD Improved Parton Model treating the above as a gluonic subprocess assumes that for gluons in the nucleon $p^{2}$ is small i.e. $p^{2} \leq M^{2}$.

The analogue of (8.1.2) for the contribution of Fig. 8.2 to the scaling function $g_{1}^{p}(x)$ is, for 3 massless flavours,

$$
g_{1}^{p}(x)=\frac{1}{3} \int_{x}^{1} \frac{d x^{\prime}}{x^{\prime}} \Delta g\left(x^{\prime}\right) g_{1}^{\gamma^{*} g}\left(x / x^{\prime}\right)
$$

Taking the first moment of the convolution (8.1.5) we obtain for the contribution of Fig. 8.2 to $\Gamma_{1}^{p}$ (for 3 flavours)

$$
\Gamma_{1}^{p}=\frac{1}{3}\left[\int_{0}^{1} d x \Delta g(x)\right]\left[\int_{0}^{1} d x g_{1}^{\gamma^{*} g}(x)\right]
$$

and using (8.1.4) one finds exactly the contribution (6.3.11) due to the anomaly.

\subsection{Study of the gluonic contribution to $g_{1}(x)$ and the definition of $\Delta g(x)$}

However, some caution is necessary about the interpretation of these results because they are based on perturbation theory, so may not be reliable as regards contributions coming from small $k_{T}$. In the small $k_{T}$ region, with $p^{2}$ limited, the momentum $p^{\prime}$ carried by the quark propagator in Fig. 8.2 is such that $\left(p^{\prime}\right)^{2} \lesssim \mathcal{O}\left(\Lambda_{\mathrm{QCD}}^{2}\right)$ so cannot be treated perturbatively. Moreover for $\left(p^{\prime}\right)^{2}$ of this scale, the contribution should be considered as due to the interaction of the virtual photon with the quark-parton of momenta $p^{\prime}$. This feature is a common one in the QCD Improved Parton Model. It has nothing specific to do with the polarized case. And the same diagram, Fig. 8.2, of course contributes in the unpolarized case, where the contribution of small $k_{T}$ is absorbed into a re-definition of the quark distribution function $q(x)$, rendering it $Q^{2}$-dependent i.e. one then deals with functions $q\left(x, Q^{2}\right)$ which evolve as $Q^{2}$ varies. 
In the unpolarized case the situation is uncontroversial. One finds, for the Born + first order terms, a result whose structure symbolically is of the form

$$
\begin{aligned}
\{1 & \left.+\alpha_{s} \ln \left(Q^{2} / p^{2}\right)+\alpha_{s} f_{q}(x)\right\} \otimes q(x) \\
& +\left\{\alpha_{s} \ln \left(Q^{2} / p^{2}\right)+\alpha_{s} f_{g}(x)\right\} \otimes g(x)
\end{aligned}
$$

where $q(x)$ and $g(x)$ are unpolarized quark and gluon distribution functions. In LLA we neglect the terms $\alpha_{s} f_{q}(x)$ and $\alpha_{s} f_{g}(x)$, and, correct to order $\alpha_{s}$, factorize the remaining terms by introducing a factorization scale $\mu^{2}$ :

$$
\begin{aligned}
\left\{1+\alpha_{s} \ln \left(Q^{2} / p^{2}\right)\right\} q(x) & =\left\{1+\alpha_{s} \ln \left(Q^{2} / \mu^{2}\right)+\alpha_{s} \ln \left(\mu^{2} / p^{2}\right)\right\} q(x) \\
& \approx\left\{1+\alpha_{s} \ln \left(Q^{2} / \mu^{2}\right)\right\}\left\{1+\alpha_{s} \ln \left(\mu^{2} / p^{2}\right)\right\} q(x) .
\end{aligned}
$$

We also write

$$
\alpha_{s} \ln \left(Q^{2} / p^{2}\right) g(x)=\left\{\alpha_{s} \ln \left(Q^{2} / \mu^{2}\right)+\alpha_{s} \ln \left(\mu^{2} / p^{2}\right)\right\} g(x)
$$

and then (8.2.1) becomes, to order $\alpha_{s}$,

$$
\left\{1+\alpha_{s} \ln \left(Q^{2} / \mu^{2}\right)\right\} q\left(x, \mu^{2}\right)+\alpha_{s} \ln \left(Q^{2} / \mu^{2}\right) g(x)
$$

where

$$
q\left(x, \mu^{2}\right) \equiv\left[1+\alpha_{s} \ln \left(\mu^{2} / p^{2}\right)\right] q(x)+\alpha_{s} \ln \left(\mu^{2} / p^{2}\right) g(x) .
$$

In this way the contributions sensitive to low $k_{T}$ and singular in the limit $p^{2} \rightarrow 0$ gets absorbed into new, non-perturbative distribution functions which we cannot calculate, but have to measure.

The leading logarithm $\ln Q^{2}$ comes specifically from the ultraviolet region of the $k_{T}$ integration which kinematically runs up to

$$
k_{T}^{2} \leq \frac{Q^{2}}{4} \frac{1-x}{x}
$$

It is therefore insensitive to different ways of factorizing or separating the low and high $k_{T}$ regions and there is no ambiguity about the definition of $q\left(x, \mu^{2}\right)$ in $L L A$.

The situation is different when working beyond the LLA where one does not neglect terms of the form $\alpha_{s} f(x)$ in (8.2.1). They do not uniquely arise from the large $k_{T}$ region and it is essentially a matter of taste or convention whether they or part of them are included in $q\left(x, \mu^{2}\right)$ or left as a piece of the gluon contribution. For this reason it is important, beyond the LLA, to indicate what convention is in use. Hence one uses $q\left(x, \mu^{2}\right)_{\overline{\mathrm{MS}}}, q\left(x, \mu^{2}\right)_{\mathrm{DIS}}$ etc..

In what way is the polarized case different? The key difference is the factor $(2 x-1)$ in expression (8.1.4). For it means that the usual large logarithm $\ln \left(Q^{2} / p^{2}\right)$ does not appear in the first moment, and the anomaly contribution comes from the second term $2(\ln x+1)$ in $(8.1 .4)$. But this seems to be like the terms $\alpha_{s} f(x)$ in (8.2.1) and therefore inherently ambiguous. There are many papers on this question. For access to these see e.g. [BAS 91]. We believe that there is a reasonably persuasive argument in favour of a particular resolution of the ambiguity. Our approach is similar in spirit to that of Ross and Roberts [ROS 90] and Carlitz, Collins, Mueller [CAR 88] (se also [MAN 90a]).

To study the rôle of $k_{T}$ let us introduce a lower cut-off $\Lambda$ in the $k_{T}$ integration i.e. in evaluating the diagram of Fig. 8.2 we keep $k_{T} \geq \Lambda$. The result which replaces (8.1.3) 
is, for $Q^{2} \gg \Lambda^{2}$,

$$
\begin{aligned}
g_{1}^{\gamma^{*} g}\left(x, Q^{2}\right)_{k_{T} \geq \Lambda} & =\frac{e_{f}^{2}}{2} \frac{\alpha}{2 \pi}\left\{(2 x-1)\left[\ln \left(\frac{\left.Q^{2}(1-x) / x\right)}{x(1-x) p^{2}+m_{q}^{2}+\Lambda^{2}}\right)-1\right]\right. \\
& \left.+(1-x) \frac{2 m_{q}^{2}-x(2 x-1) p^{2}}{x(1-x) p^{2}+m_{q}^{2}+\Lambda^{2}}\right\}
\end{aligned}
$$

The first term in square brackets can be written

$$
\ln \left(\frac{Q^{2}}{p^{2}} \frac{1-x}{x}\right)-\ln \left[x(1-x)+\frac{m_{q}^{2}}{p^{2}}+\frac{\Lambda^{2}}{p^{2}}\right]
$$

and we see that the entire term $\ln \left\{\left(Q^{2} / p^{2}\right)[(1-x) / x]\right\}$ and not just $\ln \left(Q^{2} / p^{2}\right)$ is insensitive to the value of $\Lambda$ and therefore can be associated with the region of large $k_{T}$. This is no surprise since the structure $Q^{2}(1-x) / x$ just reflects the upper limit of the $k_{T}$ integration (8.2.2). It thus seems clear that the $\ln [(1-x) / x]$ emerging from this term is a high $k_{T}$ effect and surely belongs to the gluon partonic sub-process, in agreement with the anomaly analysis.

Returning to the case $m_{q}=0, \Lambda=0$, we thus rewrite (8.1.4), for each flavour $f$, as

$$
g_{1}^{\gamma^{*} g}\left(x, Q^{2}\right)=\frac{e_{f}^{2}}{2} \frac{\alpha_{s}}{2 \pi}(2 x-1)\left\{\ln \left(\frac{Q^{2}}{p^{2}} \frac{1-x}{x}\right)-\ln x(1-x)-2\right\},
$$

introduce the factorization scale $\mu^{2}$ :

$$
\begin{aligned}
g_{1}^{\gamma^{*} g}\left(x, Q^{2}\right) & =\frac{e_{f}^{2}}{2} \frac{\alpha_{s}}{2 \pi}(2 x-1)\left\{\ln \left(\frac{Q^{2}}{\mu^{2}} \frac{1-x}{x}\right)+\ln \left(\mu^{2} / p^{2}\right)\right. \\
& -\ln x(1-x)-2\}
\end{aligned}
$$

and associate the first term with the gluonic contribution. The rest of the terms are then absorbed into the quark distribution $\Delta q_{f}+\Delta \bar{q}_{f}$.

Finally then, choosing $\mu^{2}=Q^{2}$, the gluonic contribution from Fig. 8.2 to $g_{1}^{p}(x)$ is, via $(8.1 .5)$

$$
\left.g_{1}^{p}(x)\right|_{\text {gluonic }}=\frac{1}{3} \frac{\alpha_{s}}{2 \pi} \int_{x}^{1} \frac{d x^{\prime}}{x^{\prime}}\left\{\left(2 \frac{x}{x^{\prime}}-1\right) \ln \left(\frac{1-x / x^{\prime}}{x / x^{\prime}}\right)\right\} \Delta g\left(x^{\prime}\right)
$$

and the modified quark contribution is

$$
\left.g_{1}^{p}\left(x, Q^{2}\right)\right|_{\text {quark singlet }}=\frac{1}{9} \Delta \Sigma\left(x, Q^{2}\right)
$$

where the contribution of Fig. 8.2 yields

$$
\begin{aligned}
\Delta \Sigma\left(x, Q^{2}\right) & =\Delta \Sigma(x)+\frac{\alpha_{s}}{2 \pi} \int_{x}^{1} \frac{d x^{\prime}}{x^{\prime}}\left(2 \frac{x}{x^{\prime}}-1\right)\left[\ln \left(Q^{2} / p^{2}\right)\right. \\
& \left.-\ln \frac{x}{x^{\prime}}\left(1-\frac{x}{x^{\prime}}\right)-2\right] \Delta g\left(x^{\prime}\right) .
\end{aligned}
$$

Of course, the first moment

$$
\Delta \Sigma=\int_{0}^{1} d x \Sigma\left(x, Q^{2}\right)
$$

remains independent of $Q^{2}$ in accordance with the discussion in Section 7.2. Clearly, the full definition of $\Delta \Sigma\left(x, Q^{2}\right)$ to LLA must also include the contribution from Fig. 8.4. 


\subsection{Measuring $\Delta g(x)$ via 2 -jet production}

It is important to note that the definition of $\Delta g(x)$ adopted above associates it with the physical process of the electroproduction of two jets with large transverse momentum $\boldsymbol{k}_{T}$ and $-\boldsymbol{k}_{T}$ respectively. Measurement of this semi-inclusive process thus gives direct access to $\Delta g(x)$. It is determined by a physical measurement and thus, manifestly, must be gauge invariant. (The formal question of gauge invariance is discussed in Section 9.3).

We have for the contribution to $g_{1}\left(x, Q^{2}\right)$ coming from the two-jet events

$$
\begin{gathered}
\gamma^{*} N \rightarrow \operatorname{jet}\left(\boldsymbol{k}_{T}\right)+\operatorname{jet}\left(-\boldsymbol{k}_{T}\right)+X \\
\left.g_{1}^{2-\text { jet }}\left(x, Q^{2}\right)\right|_{k_{T} \geq \Lambda}=\left.\frac{1}{3} \int_{x}^{1} \frac{d x^{\prime}}{x^{\prime}} g_{1}^{\gamma^{*} g}\left(x / x^{\prime}, Q^{2}\right)\right|_{k_{T} \geq \Lambda} \Delta g\left(x^{\prime}\right)
\end{gathered}
$$

where $g_{1}^{\gamma^{*} g}$ was given in (8.2.3) and the lower cut off on $k_{T}$ is given by

$$
\Lambda^{2} \gg m_{q}^{2}, p^{2}
$$

where $p^{2}$ is the 'virtuality' of the gluon.

\subsection{The shape of $g_{1}^{p}(x)$ and the anomalous gluon contribution}

We have seen that the anomalous gluonic contribution to the first moment $\Gamma_{1}^{p}$ permits an explanation of the EMC experiment in which the total spin carried by the quarks can be relatively large provided that $\Delta g=\int_{0}^{1} d x \Delta g(x)$ is big enough. For example $S_{z}^{\text {quarks }} \simeq 0.6 J_{z}^{\text {nucleon }}$ requires $\Delta g \simeq 4$ at $Q^{2} \simeq 11(\mathrm{GeV} / \mathrm{c})^{2}$.

We consider now the $x$-dependence of $g_{1}^{p}(x)$ given by (we ignore perturbative QCD corrections)

$$
\begin{aligned}
g_{1}^{p}(x) & =\frac{1}{2}\left\{\frac{4}{9}[\Delta u(x)+\Delta \bar{u}(x)]+\frac{1}{9}[\Delta d(x)+\Delta \bar{d}(x)]\right. \\
& \left.+\frac{1}{9}[\Delta s(x)+\Delta \bar{s}(x)]\right\}+\left.g_{1}^{p}(x)\right|_{\text {gluonic }}
\end{aligned}
$$

where $g_{1}^{p}(x)_{\text {gluonic }}$ is given in (8.2.6).

We saw in Section 4 that without a gluonic contribution the EMC experiment forced us to have $\Delta \Sigma \simeq 0$ and this was achieved by having a surprising large negative $\Delta s$ contribution.

It is interesting to contemplate the other extreme i.e. to take $\Delta s(x) \simeq 0$. Assuming a roughly $S U(3)$ invariant description of the sea quarks (this is not a good approximation for the unpolarized distributions) $g_{1}^{p}(x)$ will in this case depend only upon the valence quark distributions $\Delta u_{\mathrm{v}}(x)$ and $\Delta d_{\mathrm{v}}(x)$ and on $\Delta g(x)$. The normalization of the valence quark distribution is fixed by the values of F and D (see Sections 4.2 and 4.3) and their shape is constrained by the requirement

$$
\left|\Delta u_{v}(x)\right| \leq u_{v}(x) \quad\left|\Delta d_{v}(x)\right| \leq d_{v}(x) .
$$

Furthermore at $x \simeq 0.3$, where $x g_{1}^{p}(x)$ has its maximum value (see Fig. 4.2) there is essentially no contribution from $\Delta g(x)$, so that there is a further constraint on $\Delta u_{v}(x)$, $\Delta d_{v}(x)$ in this region. It then turns out that one requires a significant negative contribution near $x=0.1$ to fit the data in Fig. 4.2. The question then is: can a large gluonic term provide such a contribution? It is found empirically [ROS 90] that this is not possible as 
is demonstrated in Fig. 8.5. One can trace this failure to the feature that the factor in parenthesis in (8.2.6) has a double zero at $x^{\prime}=2 x$. Given that $\Delta g(x)$ is bounded by

$$
|\Delta g(x)| \leq g(x)
$$

and that $g(x)$ dies out rapidly as $x$ increases, the region of overlap in the convolution in (8.2.6) where both the kernel and $\Delta g(x)$ are big is a very small one and in effect the integration only samples $\Delta g\left(x^{\prime}\right)$ for a small portion of the region $x \leq x^{\prime} \leq 1$.

Of course there is no reason to suppose a total absence of a strange quark contribution. The problem with the EMC analysis without the anomalous gluon contribution was that it demanded a surprisingly large negative $\Delta s(x)$. Figure 8.6 taken from [ROS 90] shows that a reasonable compromise is possible. The fit shown corresponds, for F/D $=0.55$ (which is approximately the value used in Section 4), to having

$$
\begin{aligned}
& \Delta u=0.84 \quad \Delta d=-0.41 \quad \Delta s=-0.05 \\
& \Delta \Sigma=0.38 \quad \Delta g \simeq 3 \text {. }
\end{aligned}
$$

The exact values should not be taken too seriously since they depend upon the simple, but reasonable parametrization used for the polarized quark distributions in [ROS 90], and because the small $x$ behavior used does conform to the expectations from Regge theory. However the fit does indicate that the EMC data can be explained with a reasonable strange quark contribution, a sensible $\Delta \Sigma$ and a moderate $\Delta g$ 巴.

\subsection{The anomaly in $\nu p$-elastic scattering and weak interaction structure functions}

We mentioned in Section 6.3 that there is no anomaly in the limit $m_{q}^{2} / k^{2} \rightarrow \infty$. Analogously, we see that in the limit of $m_{q}^{2} / p^{2} \rightarrow \infty$ (8.1.3) becomes

$$
g_{1}^{\gamma^{*} g}\left(x, Q^{2}\right)=\frac{e_{f}^{2}}{2} \frac{\alpha_{s}}{2 \pi}\left\{(2 x-1)\left[\ln \left(\frac{Q^{2}}{m_{q}^{2}} \frac{1-x}{x}\right)-1\right]+2(1-x)\right\}
$$

and the first moment vanishes. In this case it is natural to consider the contribution from Fig. 8.2 as a modification of the quark distribution functions.

There is an interesting application of the rôle of quark mass in the anomaly in elastic neutrino proton scattering, which is mediated by $Z_{0}$ exchange as shown in Fig. 8.7. Since the parity-violating $Z_{0}$-quark coupling involves $\gamma^{\mu} \gamma_{5}$ Fig. 8.7 also depends upon the proton matrix element of the axial vector current. In the Naive Parton Model, for forward scattering, the contribution is, in principle, proportional to

$$
\delta q \equiv(\Delta u+\Delta \bar{u}-\Delta d-\Delta \bar{d})+(\Delta c+\Delta \bar{c}-\Delta s-\Delta \bar{s})
$$

(the sign change for the $I_{3}=-1 / 2$ quarks, compared to the electromagnetic case, is due to the $Z_{0}$ couplings).

Experimentally [AHR 87] it seems that $\delta q$ is about $12 \%$ larger than expected from the $\Delta u, \Delta d$ contribution (see also the discussion in Section 4.2.3). This has been interpreted [ELL 88] as evidence for a large negative $\Delta s$ in agreement with (4.3.17), assuming a negligible $\Delta c$. However, the anomaly also contributes to this process via the diagram of Fig. 8.8.

1) Note added in proof: Presumably with the new proton data [ADA 94] an even more reasonable $\Delta \Sigma$ and $\Delta g$ would be possible. 
By the above discussion there is no contribution from the heavy quarks, but for each massless quark, in the QCD Field Theoretic Model,

$$
\Delta q+\Delta \bar{q} \rightarrow \Delta q+\Delta \bar{q}-\frac{\alpha_{s}}{2 \pi} \Delta g .
$$

Hence, in (8.5.2)

$$
\delta q \rightarrow \delta q+\frac{\alpha s}{2 \pi} \Delta g .
$$

In the extreme case $\Delta s=0$ one then requires a rather large $\Delta g \simeq 5$ to fit the data. As discussed in Section 8.4 such a scenario leads to difficulties with the $x$-dependence of $g_{1}^{p}(x)$. But the more reasonable choice $\Delta s=-0.05, g \simeq 3$ is perfectly acceptable.

The weak interaction structure functions discussed in Section 3.5 also receive an anomalous gluonic contribution via a diagram analogous to that of Fig. 8.2, with the photon replaced by a $Z_{0}$ (neutral current) or a by $W^{ \pm}$(charged current). Such contribution leads again, for each massless quark, to the result (8.5.3) [VOG 91]; for the structure functions measured in charged current DIS, which involve diagrams with transitions from light to heavy quarks [see Eqs. (3.5.8)], there are interesting quark mass effects [VOG 91; MAT 92, 92b].

\subsection{Operator Product Expansion vs. QCD Improved Parton Model}

In Section 5 we stressed that only the fully inclusive electroproduction process could be expressed in terms of the matrix element of a product of electromagnetic currents (5.1.1) and hence only in this case could one utilize the Operator Product Expansion. Our definition of $\Delta g(x)$ has focused on the semi-inclusive large $k_{T} 2$-jet process and it might thus seem strange that our results for the first moment should agree exactly with the anomaly result obtained using the Operator Product Expansion. But this is simply a 'red herring'. We have not thrown away the part of the cross-section corresponding to small $k_{T}$. It is simply absorbed into the redefinition of the quark distribution functions. So the results of the Operator Product Expansion should agree with the QCD Improved Parton Model results.

Moreover, if we return to the triangle anomaly discussed in Section 6.3 we may ask what region of the $k_{T}$ integration involved in evaluating the triangle diagram was important for the anomaly. As stressed in [CAR 88] it is indeed the region of large $k_{T}$ that is relevant, so there is consistency between the two methods of deriving the anomaly contribution to the first moment of $g_{1}^{p}(x)$.

These considerations, as emphasized in [ROS 90], draw our attention to an important issue, namely that whatever cut-off or factorization scheme we use, we must use it consistently if we wish to compare results from the Operator Product Expansion with those of the QCD Improved Parton Model. We must also be careful when dealing with physical quantities that depend on both polarized and unpolarized distribution functions.

For example, given that $g_{ \pm}(x)$ measures the number density of gluons polarized along or opposite to the spin direction of the proton, they must be positive. Thus one expects

$$
|\Delta g(x)|=\left|g_{+}(x)-g_{-}(x)\right| \leq\left|g_{+}(x)+g_{-}(x)\right|=g(x) .
$$

But $g(x)$ is measured in unpolarized scattering and depends upon the factorization convention utilized there. Clearly (8.6.1) and analogous relations for quark distributions can only be expected to hold if the same cut-off convention is used for $\Delta g(x), g(x), \Delta q(x)$ and $q(x)$. The relevance to the phenomenology of $g_{1}^{p}(x)$ was discussed in Section 8.4. 
Finally, there is another 'red herring' in the literature which needs to be pointed out. The anomaly emerges from the triangle diagram only if $k_{T}$ is integrated up to infinity, as it must be in the Feynman diagram. It is claimed that, on the contrary, the anomaly emerges when integrating up to $k_{T}^{2} \approx Q^{2}$ in the QCD Improved Parton Model. The latter statement is misleading. True, at fixed $x, k_{T}^{2} \max \approx Q^{2}$ but to get the anomaly we must integrate over $x$ from 0 to 1 . The region $x \rightarrow 0$ corresponds, via (8.2.2), precisely to $k_{T}^{\max } \rightarrow \infty$.

In summary there is perfect agreement between the two approaches to the gluon contribution in polarized deep inelastic scattering, provided care is taken to use consistent factorization or renormalization conventions in both.

\section{$9 \quad$ Non-perturbative effects in the interpretation of the measurement of $\Gamma_{1}^{p}$}

The previous discussion has focused on the rôle of perturbative aspects of the axial anomaly in the theoretical description of polarized DIS. But the axial anomaly also has deep and non-trivial non-perturbative consequences in QCD because of the complicated topological structure of the non-abelian theory. We first provide a brief survey of the topological issues and than turn to their rôle in DIS. We show that they support the conclusions drawn from the reinterpretation of the measurement of $\Gamma_{1}^{p}$ including the effect of the anomaly. Finally we give a brief survey of the open questions in the understanding of the EMC result.

\subsection{Topological effects and the axial ghost in QCD - a pedagogical reminder}

We follow the approach of Dyakonov and Eides [DYA 81]. A more detailed pedagogical treatment can also be found in [LEA 94]. Consider a purely gluonic version of QCD, and choose for simplicity the gauge in which $A_{0}^{a}(x)=0$. While satisfying this gauge condition the theory is still invariant under arbitrary time-independent gauge tranformations

$$
\boldsymbol{A}_{j} \rightarrow \boldsymbol{U} \boldsymbol{A}_{j} \boldsymbol{U}^{-1}+\frac{i}{g}\left(\partial_{j} \boldsymbol{U}\right) \boldsymbol{U}^{-1}
$$

where, as usual, $\boldsymbol{A}_{j}$ without a colour label stands for the matrix $\left(\boldsymbol{\lambda}^{a} / 2\right) A_{j}^{a}$ and $\boldsymbol{U}=\boldsymbol{U}(\boldsymbol{x})$ only and where, for a consistent theory, one utilises transformation such that $\boldsymbol{U}(\boldsymbol{x}) \rightarrow \boldsymbol{I}$ as $|\boldsymbol{x}| \rightarrow \infty$.

The fields $A_{j}^{a}$ play the rôle of generalized coordinates in the quantum theory and the rôle of generalized momenta is played by

$$
\pi_{a}^{j}(x) \equiv \frac{\partial \mathcal{L}}{\partial \dot{A}_{j}^{a}}=-\dot{A}_{a}^{j}=E_{j}^{a}
$$

where $\boldsymbol{E}^{a}$ is the colour-electric field.

The generalized coordinates and momenta satisfy the usual canonical equal time commutation relations

$$
\left[\pi_{i}^{a}(\boldsymbol{x}, t), A_{j}^{b}(\boldsymbol{y}, t)\right]=-\delta_{i j} \delta_{a b} \delta^{3}(\boldsymbol{x}-\boldsymbol{y}) .
$$

The Hamiltonian, quite analogous to QED, turns out to be

$$
H=\int d^{3} \boldsymbol{x} \operatorname{Tr}\left[\boldsymbol{E}^{2}+\boldsymbol{B}^{2}\right]
$$


where the components of the colour-magnetic field $\boldsymbol{B}^{a}$ are defined by

$$
B_{j}^{a}=\frac{1}{2} \varepsilon_{j k l} G_{a}^{k l} .
$$

Unlike QED, the fields do change under an arbitrary gauge transformation:

$$
\boldsymbol{E}_{j} \rightarrow \boldsymbol{U} \boldsymbol{E}_{j} \boldsymbol{U}^{-1} \quad \boldsymbol{B}_{j} \rightarrow \boldsymbol{U} \boldsymbol{B}_{j} \boldsymbol{U}^{-1}
$$

Bearing in mind that $\boldsymbol{E}^{2}=\dot{\boldsymbol{A}}^{2}$ we see that the first term in (9.1.4) plays the rôle of the kinetic energy whereas $V \equiv \operatorname{Tr}\left[\boldsymbol{B}^{2}\right]$ represents the potential energy.

The analogue of a wave function in ordinary quantum mechanics is here a functional $\Psi[\boldsymbol{A}(x)]$ of the fields and the stationary states and energies are found from

$$
H \Psi=E \Psi
$$

Now the gauge transformations $\boldsymbol{U}(\boldsymbol{x})$ fall into discrete classes labelled by an integer $n$, the winding number. The class with $n=0$ are called small gauge tranformations and can be built up from the identity by a series of infinitesimal transformations - by smoothly varying the parameters which specify them they can be continously deformed into the identity transformation. The classes with $n \neq 0$ are called large gauge transformations and cannot be continuously transformed into the identity transformation (for an elementary discussion see [LEA 94]).

In Section 6.4 we introduced the gluon axial current $K^{\mu}$ [see (6.4.1)] which is a function of the fields $A_{j}^{a}$. Based on this we can construct a new variable $X$, a function of the fields $A_{j}^{a}$, (often called a collective coordinate):

$$
X \equiv \frac{g^{2}}{16 \pi^{2}} \int d^{3} \boldsymbol{x} K_{0}(x)
$$

and such that it changes by the integer $n$ when the fields undergo a gauge transformation of winding number $n$ but does not change under small gauge transformation. In fact

$$
X \rightarrow X+\frac{1}{24 \pi^{2}} \int d^{3} \boldsymbol{x} \varepsilon_{i j k} \operatorname{Tr}\left[\left(\partial_{i} \boldsymbol{U}\right) \boldsymbol{U}^{-1}\left(\partial_{j} \boldsymbol{U}\right) \boldsymbol{U}^{-1}\left(\partial_{k} \boldsymbol{U}\right) \boldsymbol{U}^{-1}\right]
$$

and the change in $X$ can be shown to be just $n$. Thus

$$
X \rightarrow X+n
$$

Note that the non-vanishing of the trace term (9.1.9) is a consequence of the nonAbelian nature of QCD. For normal QED, (i.e. in 4-dimensional Minkowski space) on the contrary, $U=e^{i \Lambda(\boldsymbol{x})}$ and the trace term vanishes.

Now we can imagine expressing the potential energy $V$ in terms of $X$ and any other necessary variables. If then we plot $V$ vs. $X$ it must have the same value at all values $X_{n}=X+n$, since it is, via (9.1.6), invariant under all gauge transformations, large or small. In other words if we express the potential in terms of $X$ and the other required variables, $V=V(X, Y, \ldots)$, then $V$ is a periodic function of $X$ with period 1 as shown in Fig. 9.1 This periodicity was first noticed by Faddeev [FAD 76], Jackiw and Rebbi [JAC 76], 't Hooft ['THO 76] and Callan, Dashen and Gross [CAL 76].

Before exploring the consequences it may help to examine a very simple example of the above phenomenon. 
Consider electrodynamics in one time and one space dimension, where $A^{\alpha}=\left(A_{0}, A_{x}\right)$, and define $K_{\alpha}=\varepsilon_{\alpha \beta} A^{\beta},\left(\varepsilon_{01}=-\varepsilon_{10}=1\right)$ so that $K_{0}=A_{x}$.

Let

$$
X=\frac{g}{2 \pi} \int_{-\infty}^{\infty} d x K_{0}(x)=\frac{g}{2 \pi} \int_{-\infty}^{\infty} d x A_{x}(x)
$$

Then, under the gauge transformation $U=e^{i \phi(x)}$,

$$
A_{x} \rightarrow A_{x}+\frac{i}{g}\left(\frac{\partial}{\partial x} U\right) U^{-1}=A_{x}-\frac{1}{g} \frac{d \phi}{d x}
$$

Consequently

$$
X \rightarrow X-\frac{1}{2 \pi} \int_{-\infty}^{\infty} d x \frac{d \phi}{d x}=X-\frac{1}{2 \pi}\{\phi(\infty)-\phi(-\infty)\}
$$

But the requirement $U(x) \rightarrow 1$ at spatial infinity means that $\phi(\infty)$ and $\phi(-\infty)$ must both be multiples of $2 \pi$, but not necessarily the same multiples. Hence $X \rightarrow X+$ (an integer $n)$.

Returning to QCD we see that we are dealing with a quantum problem with periodic potential i.e. an analogue of an electron in a crystal lattice. The energy spectrum will thus be a band spectrum. Now, near the bottom of the lowest energy band the electron behaves like a free particle with $E=k^{2} / 2 m^{*}$ and $m^{*}$ an effective mass which depends on the details of the shape of the potential. As a consequence the Green's function $\langle 0|T[x(t) x(0)]| 0\rangle$ has the property that

$$
\left.\int d t e^{i \omega t}\langle 0|T[x(t) x(0)]| 0\rangle\right|_{\omega \rightarrow 0} \longrightarrow \frac{i}{\omega^{2} m^{*}}
$$

like the propagator of a free particle凹. The electron can move freely in the lattice. Note that these results are inherently non-perturbative.

Analogously in QCD this means that the vacuum state wave function is not localized in $X$ at one of the minima $X=$ integer, but that the ground state is spreat out in $X$ in the form of a Bloch wave

$$
|\theta\rangle=\sum_{n} e^{i n \theta}|n ; \ldots\rangle
$$

where the state $|n ; .$.$\rangle is approximately localized at the point X=n$, and the value of $\theta$ which specifies the lowest energy states is beyond our power of calculation. The above phenomenon occurs because the barriers between the different minima of the potential can be penetrated. In QCD the instantons, which are solutions of the Euclidean version of the theory, with finite actions, allow one to construct a semi-classical approximation to $|\theta\rangle$, but the structure (9.1.12) is a general result, independent of any consideration of instantons. In the following the vacuum state $|\theta\rangle$ is to be understood as the true QCD vacuum.

Analagously to (9.1.11) the Green's function involving $X(t) X(0)$ will have a pole at $\omega=0$. Bearing in mind how $X$ is constructed from $K_{0}$, we expect to find a massless pole

1) This is an immediate consequence of the equation for the Green's function of a free particle

$$
m \frac{d^{2}}{d t^{2}}\langle 0|T[x(t) x(0)]| 0\rangle=i \delta(t)
$$


in the momentum space Feynman propagator for $K_{\mu}(x)$, which is the Fourier transform of $\left\langle 0\left|T\left[K_{\mu}(x) K_{\nu}(0)\right]\right| 0\right\rangle$. To avoid a plethora of constants, let us introduce

$$
\widetilde{K}_{\mu}(x) \equiv \frac{N_{f} \alpha_{s}}{2 \pi} K_{\mu}(x)
$$

where $N_{f}$ is the number of light flavours -3 in our case. We thus expect to have for the $\widetilde{K}_{\mu}$ propagator:

$$
\int d^{4} x e^{i q \cdot x}\left\langle 0\left|T\left[\widetilde{K}_{\mu}(x) \widetilde{K}_{\nu}(0)\right]\right| 0\right\rangle=-i \Upsilon^{4} \frac{\left(g_{\mu \nu}-q_{\mu} q_{\nu} / q^{2}\right) \beta+q_{\mu} q_{\nu} / q^{2}}{q^{2}+i \epsilon}
$$

where $\Upsilon$ and $\beta$ are constants.

Some comments are necessary about the above expression. The above form will hold in any covariant gauge but the value of $\beta$ will be gauge-dependent. The fact that $\partial_{\mu} K^{\mu}(x)$ is gauge-invariant implies that the longitudinal part of the propagator (9.1.14), i.e. the part proportional to $q_{\mu} q_{\nu} / q^{2}$, must be gauge-invariant. Thus the value of $\Upsilon$ cannot depend upon the gauge and any gauge-dependence associated with the propagator can only arise from an explicit dependence upon $\beta$.

The massless ghost state thus introduced will be referred to as the 'axial ghost'. The 'ghost state' exists in an unphysical sector of the Hilbert space. (Recall that in quantizing a gauge theory the physical states form only a subspace of Hilbert space). Finally note that the coupling of the ghost will be such that the apparent $1 / q^{4}$ double pole never actually occurs and, indeed, such that no physical matrix element has a pole at $q^{2}=0$.

Notice that aside from the normalization factor, the LHS of (9.1.14) corresponds

exactly to the momentum space Feynman propagator for the 'field' $\widetilde{K}_{\mu}(x)$. We shall introduce the shorthand notation

$$
\langle T[A B]\rangle_{q} \equiv \int d^{4} x e^{i q \cdot x}\langle 0|T[A(x) B(0)]| 0\rangle
$$

for these momentum space propagators.

Equation (9.1.14) implies that in QCD there exists a zero mass axial vector ghost particle which couples to the axial gluon current $K_{\mu}$. The necessity for the ghost pole in QCD was recognised as early as 1975 by Kogut and Suskind [KOG 75]. The ghost pole was invoked by Veneziano [VEN 79] to resolve the $U(1)$ problem.

\subsection{A reminder about the $U(1)$ problem}

Although we shall not discuss the resolution of the problem we give a brief reminder as to what exactly the problem is.

As mentioned in Section 6.3 the QCD Lagrangian in the chiral limit $m_{q} \rightarrow 0$ seems to contain an additional symmetry, namely that it appears to be invariant under separate flavour transformations on the left and right-handed parts of the quark fields. Such a symmetry would lead to a parity doubling of the hadron spectrum if the vacuum were invariant under chiral transformations and therefore unique. For example, it would imply a $(J)^{P}=(1 / 2)^{-}, I=1 / 2$ partner for the nucleon. Experimentally the nearest resonance with these quantum numbers to the nucleon is the $N(1535)$, some $600 \mathrm{MeV}$ heavier than the nucleon. Now the quark mass parameters in the Lagrangian are believed to be small $\left(m_{u}, m_{d}<10 \mathrm{MeV} / \mathrm{c}^{2}\right.$ and $\left.m_{s} \simeq 150 \mathrm{MeV} / \mathrm{c}^{2}\right)$ on the scale of hadron masses, so the chiral limit should be a reasonable approximation. It is then difficult to believe that the 
non-zero quark masses are responsible for the mass gap between the nucleon and the $N(1535)$.

It is thus assumed that the chiral symmetry is broken principally as a result of a spontaneous breaking mechanism which gives rise, for example, to a quark-antiquark condensate. There is no longer a unique vacuum, but a continuum of states related to each other by chiral rotations and the symmetry is broken by choosing one of these as the true vacuum state. As a consequence there should exist a zero mass pseudoscalar Goldstone boson. Its mass would be strictly zero in the limit $m_{q}=0$ and would be expected to be non-zero, but small, in the realistic case of small quark masses. There is one Goldstone boson for each broken generator of the original symmetry.

Since the QCD Lagrangian is, in fact, invariant under $U(3)$ transformations of flavour there ought to exist nine light pseudoscalars whose masses would be zero if the $m_{q}$ were all zero. The obvious pseudoscalar nonet is $\left(\pi, K, \eta, \eta^{\prime}\right)$. In the following we shall treat the $\eta$ as the eigth member of a pure $S U(3)$ octet, and the $\eta^{\prime}$ as a pure $S U(3)$ singlet. (In reality there is a small amount of mixing which we ignore since it has no bearing on the arguments to follow).

It turns out, however, that the mass of the $\eta^{\prime}(958)$ is much larger than expected on theoretical grounds. The other 8 pseudoscalars, associated with the breaking of chiral $S U(3)_{F}$, have acceptable masses, so it is only the Goldstone boson associated with $U(1)_{F}$ that is in difficulty. It is this that is referred to as the ' $U(1)$ problem'.

\subsection{The axial ghost}

It turns out that simply introducing an axial ghost pole via (9.1.14) leads to a result for the $\eta^{\prime}$ mass which is inconsistent in the chiral limit. The correct procedure seems to be the following:

i) The above ghost is considered as a 'bare' ghost.

ii) One introduces a quark-antiquark pseudoscalor flavour singlet field $\eta_{0}(x)$ corresponding to the 'particle'

$$
\left|\eta_{0}\right\rangle \equiv \frac{1}{\sqrt{3}}|u \bar{u}+d \bar{d}+s \bar{s}\rangle
$$

which would have corresponded to the $\eta^{\prime}$ if there were no axial anomaly. The $\eta_{0}$ has a mass $m_{0}$ similar to the other pseudoscalor mesons.

iii) We introduce a direct coupling between the ghost field and $\eta_{0}(x)$ which results in mixing between the bare ghost and the $\eta_{0}$ and then study the consequences of the interaction.

To this end we introduce the bare massless ghost field $G_{\mu}^{0}(x)$ which couples directly to $\widetilde{K}_{\mu}$ so as to induce the mass zero pole in the $\widetilde{K}_{\mu}$ propagator $(9.1 .14)$. The

non-perturbative transition amplitude for the $\widetilde{K}_{\mu}-G_{\mu}^{0}$ coupling will be indicated graphically as in Fig. 9.2. The bare ghost propagator is then given by (9.1.14) without the factor $\Upsilon^{4}$. The coupling of the ghost to the $\eta_{0}$ will be described by an effective Lagrangian term $-\Delta m G_{\mu}^{0}(x) \partial^{\mu} \eta_{0}(x)$ where $\Delta m$ is a coupling constant that will play the rôle of a mass correction. This induces the diagrammatic vertices shown in Fig. 9.3.

The bare $G_{\mu}^{0}$ and $\eta_{0}$ now mix and the mass eigenstates have to be found by diagonalizing their propagators. The simplest method is by summing the diagrams, Fig. 9.4, generated by the interaction. One finds for the masses of the renormalized ghost $G_{\mu}$ and the renormalized physical $\eta^{\prime}$

$$
m_{G}=0 \quad m_{\eta^{\prime}}^{2}=m_{0}^{2}+(\Delta m)^{2} .
$$


The extra term $(\Delta m)^{2}$ can be adjusted to raise $m_{\eta^{\prime}}$ well above the typical pseudoscalar mass $m_{0}$ and this resolves the $\mathrm{U}(1)$ problem.

We can estimate the size of the parameter $\Upsilon$ involved in the coupling of $\widetilde{K}_{\mu}$ and $G_{\mu}^{0}$ as follows.

Consider the divergence of the flavour singlet axial current. We have

$$
\partial^{\mu} J_{5 \mu}^{0}=P(x)+\partial^{\mu} \widetilde{K}_{\mu}(x)
$$

where

$$
P(x)=2 i \sum_{f} m_{f} \bar{q}_{f} \gamma_{5} q_{f}
$$

The current $J_{5 \mu}^{0}-\widetilde{K}_{\mu}$ is divergenceless in the chiral limit and is thus analogous to the flavour non-singlet currents. We thus define $f_{0}$ by

$$
\left\langle 0\left|J_{5 \mu}^{0}-\widetilde{K}_{\mu}\right| \eta_{0}\right\rangle=i \sqrt{3} f_{0} q_{\mu}
$$

and expect to have $f_{0} \approx f_{\pi}=135 \mathrm{MeV}$. Now one can show that the parameter $\Upsilon$ is related to $f_{0}$ and $\Delta m$ via

$$
\Upsilon^{2}=\sqrt{3} f_{0} \Delta m
$$

The value of $m_{0}$ can be obtained from the magnitude of the quark condensates $\langle\bar{q} q\rangle$ known from QCD sum rules and this leads, via (9.3.2) and (9.3.6) to the estimate $\Upsilon \simeq$ $460 \mathrm{MeV}$ [DYA 81].

For the longitudinal part of the complete propagator for $G_{\mu}$, which we shall need later, one finds from the diagrams in Fig. 9.4a

$$
\left\langle T\left[G_{\nu} G_{\mu}\right]\right\rangle_{q}=\frac{i}{q^{2}}\left(\frac{q_{\mu} q_{\nu}}{q^{2}}\right) \frac{q^{2}-m_{0}^{2}}{q^{2}-m_{\eta^{\prime}}^{2}}
$$

and for the non diagonal $G_{\mu} \rightarrow \eta^{\prime}$ propagator given in Fig. 9.4b

$$
\left\langle T\left[\eta^{\prime} G_{\mu}\right]\right\rangle_{q}=\frac{q_{\mu}}{q^{2}} \frac{\Delta m}{q^{2}-m_{\eta^{\prime}}^{2}} .
$$

We see, therefore, that the axial ghost plays an essential rôle in resolving the $U(1)$ problem in QCD. A more sophisticated treatment, allowing for some breaking of $S U(3)_{F}$ does not affect the above results significantly, but does lead to a successful calculation of the mixing between the singlet $\left|\eta_{0}\right\rangle$ and the eighth member of the octet $\left|\eta_{8}\right\rangle$.

Finally we note that there is no danger of the production of the axial ghost as a physical particle. The cross-section for such production would have the form

$$
d \sigma_{G} \propto \mathcal{M}_{\mu} \mathcal{M}_{\nu}^{*} \varepsilon^{\mu}(\lambda)\left[\varepsilon^{\nu}(\lambda)\right]^{*} \delta\left(k^{2}\right) d^{4} k
$$

where $\mathcal{M}_{\mu}$ is the Feynman amplitude and $\varepsilon^{\mu}(\lambda)$ the polarization vector for a ghost of helicity $\lambda$ and momentum $k$. As long as the axial ghost coupling to physical particles is of the derivative type we will always have $\mathcal{M}_{\mu} \propto k_{\mu}$. The cross-section will thus involve $k^{\mu} k^{\nu} \varepsilon_{\mu}(\lambda)\left[\varepsilon_{\nu}(\lambda)\right]^{*} \delta\left(k^{2}\right)$. But for a physical particle $k^{\mu} \varepsilon_{\mu}(\lambda)=0$ and the cross-section vanishes. 


\subsection{Rôle of the ghost in the nucleon spin problem}

We showed in Section 6.4 that the nucleon expectation value of $K_{\mu}$ plays a direct rôle in polarized deep inelastic scattering. In Eq. (6.4.8) we calculated perturbatively the value of this matrix element. Now, because of the coupling of $K_{\mu}$ to the non-perturbative axial ghost, there appears to be the possibility of, in addition to the RHS of (6.4.8), a purely non-perturbative contribution. Similar reasoning applies to matrix elements of $\widetilde{J}_{5 \mu}^{0}$ [see (6.4.4)] which, perturbatively, was interpreted as the contribution of the quark spin. We shall now see how this leads to a new relation for $\Delta \Sigma$.

In any covariant gauge the nucleonic matrix elements of $\widetilde{K}_{\mu}$ must have the following general form:

$$
\left\langle P^{\prime}, \lambda^{\prime}\left|\widetilde{K}_{\mu}(0)\right| P, \lambda\right\rangle=\bar{u}_{\lambda^{\prime}}\left(P^{\prime}\right)\left[\gamma_{\mu} \widetilde{\mathcal{K}}_{1}\left(q^{2}\right)+q_{\mu} \widetilde{\mathcal{K}}_{2}\left(q^{2}\right)\right] \gamma_{5} u_{\lambda}(P)
$$

where $\widetilde{\mathcal{K}}_{1,2}\left(q^{2}\right)$ are scalar form factors, $q_{\mu}=P_{\mu}^{\prime}-P_{\mu}$ and $\lambda, \lambda^{\prime}$ are helicities.

We shall now show that the ghost contributes only to $\widetilde{\mathcal{K}}_{2}\left(q^{2}\right)$. The relevant Feynman diagrams are shown in Fig. 9.5 where the propagators are the complete propagators shown in Figs. 9.4a, b.

For the interaction of the $\eta_{0}$ with the nucleon we take a standard pseudoscalar coupling term in the Lagrangian: $-i g_{\eta_{0} N \bar{N}} \bar{\psi}_{N} \gamma_{5} \psi_{N} \eta_{0}$ and for the coupling of $G_{\mu}^{0}$ a term $-i g_{G_{0} N \bar{N}} G_{\mu}^{0} \partial^{\mu}\left(\bar{\psi}_{N} \gamma_{5} \psi_{N}\right)$, chosen so that ghosts cannot be emitted as physical particles. These give rise to the Feynman vertices shown in Fig. 9.6.

The diagrams of Fig. 9.5 yield a contribution to $\left\langle P^{\prime}, \lambda^{\prime}\left|\widetilde{K}_{\mu}(0)\right| P, \lambda\right\rangle$ of

$$
\frac{q_{\mu}}{q^{2}} \bar{u} \gamma_{5} u \frac{\Upsilon^{2}}{m_{\eta^{\prime}}^{2}}\left(\Delta m g_{\eta_{0} N \bar{N}}-m_{0}^{2} g_{G_{0} N \bar{N}}\right) .
$$

Hence, comparing with (9.4.1) the ghost contributes only to $\widetilde{\mathcal{K}}_{2}$ :

$$
\widetilde{\mathcal{K}}_{2}^{\text {ghost }}\left(q^{2}\right)=\frac{\Upsilon^{2}}{q^{2} m_{\eta^{\prime}}^{2}}\left(\Delta m g_{\eta_{0} N \bar{N}}-m_{0}^{2} g_{G_{0} N \bar{N}}\right),
$$

i.e. the ghost induces a pole in $\widetilde{\mathcal{K}}_{2}\left(q^{2}\right)$ at $q^{2}=0$. Since no other contribution can do this, (9.4.3) becomes an exact expression for $\widetilde{\mathcal{K}}_{2}\left(q^{2}\right)$ as $q^{2} \rightarrow 0$.

Note that the gauge-dependent parameter $\beta$ [see (9.1.14)] in the ghost propagator does not appear in (9.4.3). Thus the value of $\widetilde{\mathcal{K}}_{2}\left(q^{2}\right)$ as $q^{2} \rightarrow 0$, as given by (9.4.3), must be gauge-invariant.

Now the gluonic contribution that was calculated pertubatively in Section 6 occurs only in $\widetilde{\mathcal{K}}_{1}\left(q^{2}\right)$. From (6.4.8) one sees that

$$
\widetilde{\mathcal{K}}_{1}(0)=-\frac{3 \alpha_{s}}{2 \pi} \Delta g .
$$

We are now able to give the formal argument for the gauge-invariance of $\Delta g$. We know that $\partial_{\mu} K^{\mu}$ is gauge-invariant. But its forward matrix element just involves a linear combination of $\widetilde{\mathcal{K}}_{1}(0)$ and $\left[q^{2} \widetilde{\mathcal{K}}_{2}\left(q^{2}\right)\right]_{q^{2}=0}$ i.e. a linear combination of $\Delta g$ and other physical parameters [see (9.4.3)] which do not depend upon the gauge. Thus $\Delta g$ itself must be independent of the gauge.

Clearly the ghost will also contribute to the modified singlet current $\widetilde{J}_{5 \mu}^{0}$ defined in (6.4.4). If we put

$$
\left\langle P^{\prime}, \lambda^{\prime}\left|\widetilde{J}_{5 \mu}^{0}\right| P, \lambda\right\rangle=\bar{u}_{\lambda^{\prime}}\left(P^{\prime}\right)\left[\gamma_{\mu} \widetilde{G}_{1}\left(q^{2}\right)+q_{\mu} \widetilde{G}_{2}\left(q^{2}\right)\right] \gamma_{5} u_{\lambda}(P)
$$


then we will have, for the ghost contribution

$$
\widetilde{G}_{2}^{\text {ghost }}\left(q^{2}\right)=-\widetilde{\mathcal{K}}_{2}^{\text {ghost }}\left(q^{2}\right)
$$

and for the perturbative QCD contribution from (6.4.5) and (7.1.4)

$$
\widetilde{G}_{1}(0)=\Delta \Sigma \text {. }
$$

We shall now derive a connection between $\Delta \Sigma$, the $\eta^{\prime}$ decay constant and the $\eta^{\prime} N \bar{N}$ coupling.

Since in the Parton Model we work with massless quarks, consider the chiral limit, $m_{q} \rightarrow$ 0. Then in (9.4.3), $m_{\eta^{\prime}}^{2} \rightarrow(\Delta m)^{2}, m_{0}^{2} \rightarrow 0$ and using (9.3.6) we have for (9.4.6)

$$
\widetilde{G}_{2}\left(q^{2}\right)=-\frac{\sqrt{3}}{q^{2}} f_{0} g_{\eta_{0} N \bar{N}}+\text { non singular terms } .
$$

Now in this limit $\widetilde{J}_{5 \mu}^{0}$ is conserved. Hence

$$
0=\left\langle P^{\prime}, \lambda^{\prime}\left|\partial^{\mu} \widetilde{J}_{5 \mu}^{0}\right| P, \lambda\right\rangle=i q^{\mu}\left\langle P^{\prime}, \lambda^{\prime}\left|\widetilde{J}_{5 \mu}^{0}\right| P, \lambda\right\rangle
$$

Using (9.4.5) we have

$$
0=\bar{u}_{\lambda^{\prime}}\left(P^{\prime}\right) \gamma_{5} u_{\lambda}(P)\left[2 M \widetilde{G}_{1}\left(q^{2}\right)+q^{2} \widetilde{G}_{2}\left(q^{2}\right)\right] .
$$

Hence

$$
\widetilde{G}_{1}\left(q^{2}\right)=-\frac{q^{2}}{2 M} \widetilde{G}_{2}\left(q^{2}\right)
$$

and via $(9.4 .8)$

$$
\widetilde{G}_{1}\left(q^{2}\right)=\frac{\sqrt{3} f_{0} g_{\eta_{0} N \bar{N}}}{2 M}+\mathcal{O}\left(q^{2}\right)
$$

Going to $q^{2}=0$ and using (9.4.7) we obtain [VEN 89, EFR 90]

$$
\Delta \Sigma=\frac{\sqrt{3} f_{0} g_{\eta_{0} N \bar{N}}}{2 M}
$$

known as a Generalized Golberger-Treiman relation (TGV in [VEN 89]).

Note that the form of (9.4.13) differs from that in [VEN 89]. In [VEN 89] one starts with the matrix element of $J_{5 \mu}^{0}$ expressed like (9.4.5) in terms of scalar functions $G_{1,2}\left(q^{2}\right)$ :

$$
\left\langle P^{\prime}, \lambda^{\prime}\left|J_{5 \mu}^{0}\right| P, \lambda\right\rangle=\bar{u}_{\lambda^{\prime}}\left(P^{\prime}\right)\left[\gamma_{\mu} G_{1}\left(q^{2}\right)+q_{\mu} G_{2}\left(q^{2}\right)\right] \gamma_{5} u_{\lambda}(P)
$$

[note that $G_{1}(0)$ is then identical to $a_{0}$ used in Section 4.2 and Section 6] and one finds

$$
G_{1}(0)=\frac{\sqrt{3}}{2 M} f_{\eta^{\prime}}\left(g_{\eta^{\prime} N \bar{N}}-\Gamma_{Q N \bar{N}}\right) .
$$

In this form $\Gamma_{Q N \bar{N}}$ is the total contribution from gluonic effects, both from the ghost and from the gluon-parton, and of course $G_{1}(0)$ involves not just $\Delta \Sigma$ but $\Delta \Sigma-$ $\left(3 \alpha_{s} / 2 \pi\right) \Delta g$. A renormalization group analysis then leads [SHO 92] to the identification of $\Delta \Sigma$ with the first term on the RHS of (9.4.15). However, it seems to us that there is some point in separating the ghost and gluon-parton contributions. The former is totally nonperturbative and non-Abelian and is absent in QED. The latter is partially perturbative 
and would be present also in an Abelian theory. Further, the two contributions have a significantly different kinematical structure in the matrix element of $K_{\mu}$ and so can, at least in principle, be measured separately. Obviously one learns about $\Delta g$ in deep inelastic scattering; but also in various high $p_{T}$ processes, as discussed in Section 11. And it may be possible to study ghost effects in $N N$ spin dependent processes in the Regge region, as explained below.

The result (9.4.13) is unchanged if we allow for a non-zero, but flavour symmetric quark mass. Moreover it can be generalized [EFR 91] to allow for both $S U(3)_{F}$ breaking and isospin breaking. The change is small provided that allowance is made both for breaking of the equality of the quark masses and for $\pi^{0}-\eta-\eta^{\prime}$ mixing. If the latter is neglected one obtains large and misleading corrections, even the wrong sign for the gluonic contribution [CHE 89]! There is a significant cancellation between the effects of the two types of symmetry breaking, as is discussed in detail in [EFR 91].

Now (9.4.13) is interesting because it suggests, in principle, and independent measurement of $\Delta \Sigma$. The coupling of $\eta^{\prime}$ to nucleons can be obtained from studying the rôle of $\eta^{\prime}$-exchange in nucleon-nucleon elestic scattering. A phase shift analysis in the framework of a one boson exchange potential model, leads to $g_{\eta^{\prime} N \bar{N}} \simeq 7.3$ with, presumably, rather large uncertainty [DUM 83]. But in this analysis there was no ghost exchange. So the measured coupling does not really correspond to the $g_{\eta_{0} N \bar{N}}$ in (9.4.13). The ghost would generate a contact NN potential and for small $t,|t| \ll m_{\eta^{\prime}}^{2}$, what is measured is actually $\sqrt{g_{\eta_{0} N \bar{N}}^{2}-m_{\eta^{\prime}}^{2} g_{G N \bar{N}}^{2}}$ and not $g_{\eta_{0} N \bar{N}}$, but, as we shall argue, it seems likely that $\left|m_{\eta^{\prime}} g_{G N \bar{N}}\right| \ll g_{\eta_{0} N \bar{N}}$.

Now $g_{\eta^{\prime} N \bar{N}}$ can also be estimated from $\eta^{\prime} \rightarrow 2 \gamma$ decay. The decay rate has been calculated using a triangle diagram made up of the baryon octet, and assuming, as happens for $\pi^{\circ} \rightarrow 2 \gamma$, that this gives the same result as a calculation based on quark triangles [BAG 90]. This yields $g_{\eta^{\prime} N \bar{N}}=6.3 \pm 0.4$.

Both the quoted values are close to the $S U(6)$ value [TOR 84], $g_{\eta^{\prime} N \bar{N}} \simeq 6.5$. We see that there will be consistency between all these values provided that $m_{\eta^{\prime}} g_{G_{0} N \bar{N}}$ is small enough.

It may be helpful to note that there is an analogous situation suggesting a small ghost coupling. In the calculation of the 2-photon matrix element of $\widetilde{J}_{5 \mu}^{0},\left\langle\gamma\left(k_{1}\right) \gamma\left(k_{2}\right)\left|\widetilde{J}_{5 \mu}^{0}\right| 0\right\rangle$ the conservation is broken by a contribution from the QED anomaly i.e. there is an additional term $(\alpha=1 / 137)$ :

$$
\partial^{\mu} \widetilde{J}_{5 \mu}^{0}=\ldots+\frac{\alpha}{4 \pi} N_{c} \sum_{f} e_{f}^{2} \varepsilon_{\mu \nu \rho \sigma} F^{\mu \nu} F^{\rho \sigma}
$$

where $N_{c}=$ number of colours, $e_{f}=$ quark charge in units of $e$ and $F^{\mu \nu}$ is the electromagnetic field tensor. Of course, in our previous strong interaction considerations, we neglected the RHS of (9.4.16) altogether. Here, because we are dealing with photons, it is this term, and not the QCD anomaly, that is relevant. Now the above matrix element of $\widetilde{J}_{5 \mu}^{0}$ is proportional to $g_{\eta^{\prime} \gamma \gamma}$ (directly related to the rate $\eta^{\prime} \rightarrow 2 \gamma$ ) and earlier studies [ROE 90] showed consistency with (9.4.16) at the $10 \%$ level. But recently it was realised [VEN 92] that the ghost should also contribute. This has the effect of replacing $g_{\eta^{\prime} \gamma \gamma}$ by $g_{\eta^{\prime} \gamma \gamma}-m_{\eta^{\prime}} g_{G \gamma \gamma}$ and the earlier consistency of (9.4.16) implies that $m_{\eta^{\prime}} g_{G \gamma \gamma}$ must be $\leq 5 \%$ of $g_{\eta^{\prime} \gamma \gamma}$.

The smallness of the ghost coupling to quarks in both the $N \bar{N}$ and $\gamma \gamma$ case is linked to the success of the OZI approximation. It would also be expected to be small in the 
$1 / N_{c}$ expansion [VEN 92], where for any state $X$,

$$
\frac{m_{\eta^{\prime}} g_{G X X}}{g_{\eta^{\prime} X X}} \sim \frac{1}{\sqrt{N_{c}}}
$$

but this does not explain why it is as small as it seems to be.

In summary it thus seems not unreasonable to assume

$$
m_{\eta^{\prime}} g_{G_{0} N \bar{N}} \ll g_{\eta_{0} N \bar{N}}
$$

and thus

$$
g_{\eta_{0} N \bar{N}} \simeq 6-7 \text {. }
$$

Returning to (9.4.13) we take $f_{0} \approx f_{\pi}=135 \mathrm{MeV}$ and find

$$
\Delta \Sigma \simeq 0.8
$$

This supports the interpretation of the EMC result as a cancellation between a significant $\Delta \Sigma$ and a large gluon contribution as discussed in Section 7.1. It is in agreement with the naive expectation that the quark spin contribution is $\simeq 1 / 2$. At low $Q^{2}$ this is the dominant contribution. At high $Q^{2}$ gluon spin and orbital angular momentum far outstrip $\Delta \Sigma$. But this should not be a cause for concern. As stressed in Section 6.1 the proton probed at large $Q^{2}$ is very different from the static proton.

It is clearly important to be able to test the validity of the above analysis by trying to measure separately $g_{\eta_{0} N \bar{N}}$ and $g_{G_{0} N \bar{N}}$. One proposal [EFR 91a] is to look for the spin-flip effects caused by the $\eta^{\prime}$ and ghost in a situation where other flip mechanisms are absent or suppressed e.g. in $A_{N N}$ or $D_{N N}$ in proton-deuteron or deuteron-deuteron scattering, in the Regge region $|t| \ll s$. The $I=1$ exchanges of $\rho$ and $\pi$ are forbidden and the $\mathbb{P}, \mathbb{P}^{\prime}, \omega$ and $f$ exchanges are predominantly non-flip. So $\eta, \eta^{\prime}$ and the ghost should be the most important exchanges. The ghost exchange presumally contributes a fixed pole in the complex- $J$ plane where the $\eta$ and $\eta^{\prime}$ are associated with moving Regge poles. Consequently the energy variation of their contributions will be quite different in character and this should become discernable at high energies.

\subsection{Attempts to provide a physical interpretation of the EMC result}

The result of the EMC experiment can be summarized as the statement that [see

$$
\left|a_{0}\right|=\left|\Delta \Sigma-3 \frac{\alpha_{s}}{2 \pi} \Delta g\right| \ll 0 .
$$

Now from (6.4.2) we have

$$
\left\langle P^{\prime}, \lambda^{\prime}\left|\partial^{\mu} \widetilde{K}_{\mu}\right| P, \lambda\right\rangle=-3 \frac{\alpha_{s}}{\pi}\left\langle P^{\prime}, \lambda^{\prime}\left|\boldsymbol{E}^{a} \cdot \boldsymbol{B}^{a}\right| P, \lambda\right\rangle
$$

where $\boldsymbol{E}^{a}$ and $\boldsymbol{B}^{a}$ are the colour-electric and magnetic fields, and from (9.4.11) the LHS is

$$
\left[2 M \widetilde{\mathcal{K}}_{1}\left(q^{2}\right)+q^{2} \widetilde{\mathcal{K}}_{2}\left(q^{2}\right)\right] \bar{u}_{\lambda^{\prime}}\left(P^{\prime}\right) \gamma_{5} u_{\lambda}(P) .
$$

Consider now the term in square brackets as $q^{2} \rightarrow 0$. From $(9.4 .4)$ and $(9.4 .6,8)$

$$
\begin{aligned}
\lim _{q^{2} \rightarrow 0}\left[2 M \widetilde{\mathcal{K}}_{1}\left(q^{2}\right)+q^{2} \widetilde{\mathcal{K}}_{2}\left(q^{2}\right)\right] & =-2 M \frac{3 \alpha_{s}}{2 \pi} \Delta g+\sqrt{3} f_{0} g_{\eta_{0} N \bar{N}} \\
& =\text { [gluon-parton] }+ \text { [ghost }] .
\end{aligned}
$$


Use of the Generalized Goldberger-Treiman relation (9.4.13) then implies, via (9.5.1), that

$$
\left|\lim _{q^{2} \rightarrow 0}\left[2 M \widetilde{\mathcal{K}}_{1}\left(q^{2}\right)+q^{2} \widetilde{\mathcal{K}}_{2}\left(q^{2}\right)\right]\right| \ll 0 .
$$

In other words there seems to be a cancellation between the 'semi-perturbative' gluon-parton contribution and the non-perturbative 'ghost' contribution in (9.5.4). It remains a question of some interest as to why this compensation occurs. We consider briefly some of the physical arguments presented to explain this.

That $a_{0} \simeq 0$ was claimed to be quite natural in the Skyrme model approach to the 'spin crisis' [BRO 88]. The $S U(3)$ Skyrme model deals with an octet of pseudoscalar Goldstone bosons whose self-interactions are described by the non-linear Skyrme Lagrangian. Quark or gluon degrees of freedom do not occur explicitly and the baryons appear as topologically non-trivial soliton solutions of the equations of motion. Since there are no quark fields it is not absolutely obvious what function of the boson fields corresponds to the singlet axial current $J_{5 \mu}^{0}$. Nonetheless arguments can be given for the form of $J_{5 \mu}^{0}$ and in [BRO 88] it was claimed that $a_{0}$ is of order $1 / N_{c}$ and, in fact, $a_{0} \simeq 0$. (In these papers $a_{0}$ is identified with $\Delta \Sigma$, but that is irrelevant). However, this approach has been criticized by Ryzak [RYZ 89] who points out that [BRO 88] utilises an effective Lagrangian calculated to order $1 / N_{c}$ in a $1 / N_{c}$ expansion, yet which does not permit the $\eta^{\prime}$ to decay although such decay is precisely a $1 / N_{c}$ effect. In addition, in this approximation $m_{\eta^{\prime}} / M \propto\left(N_{c}\right)^{-3 / 2}$ which is far from reality. Inclusion of a term to allow $\eta^{\prime}$ decay would destabilize the soliton solutions. Ryzak has shown how to overcome this difficulty. He argues that there are many uncertainties in the estimation of $a_{0}$ but concludes that one can have $a_{0}=0.2 \pm 0.1$ in Skyrme type models. However it is hard to see why the $\eta^{\prime}$ couples strongly to the nucleon in these models.

An entirely different approach to the compensation is discussed in [FOR 90], where it is argued that the cancellation i.e. the validity of (9.5.5) is natural for instanton configurations of the gluon fields when there is just one flavour. But it is not at all self-evident what happens for $N_{f}=3$.

Yet another proposal [KUH 90] is that there is a link between the so-called conformal anomaly, which involves $G_{\mu \nu} G^{\mu \nu}$ and the axial anomaly, which involves $G_{\mu \nu} \widetilde{G}^{\mu \nu}$. By making an assumption about analyticity in the mass of the regulator fermion fields it is claimed that one has the following exact result in the chiral limit:

$$
a_{0}=\frac{-2 N_{f}}{11 N_{c}-2 N_{f}}=-\frac{2}{9}
$$

for 3 light flavours. Such a connection between the anomalies and the relation (9.5.6) seems quite natural in the framework of supersymmetric QCD where both the energymomentum tensor (with its conformal anomaly) and the flavour singlet current (with its axial anomaly) occur in the same supermultiplet of currents. But the extension to ordinary QCD is far from obvious, and some counter-arguments have been given [ANS 92a]. So it is not at all clear that (9.5.6) can be taken at face value. On the other hand attempts have been made [DOR 93] to support (9.5.6) on the basis of instanton dominance of the $\theta$-vacuum.

It has also been suggested [DOR 91] that instanton dominance of the QCD vacuum could generate large polarized sea quark distributions. In [DOR 91, 93], it is tacitly assumed that there is no anomalous $\Delta g$ contribution to $a_{0}$, so the EMC experiment is interpreted as implying $\Delta \Sigma \simeq 0$ and the parameters of the model are adjusted so that the po- 
larized sea cancels the standard Naive Parton Model polarized valence quark contribution. The instantons are supposed to generate an effective quark-quark interaction which mimics Pomeron exchange except that the exchanged object has $I=0, G=C=1, P=+1$ and flips helicity. By the usual connection between high energy behaviour in off-shell Compton scattering and the small- $x$ behaviour of distributions [see Section 4.1.1] it is argued that for small $x,[\Delta q(x)+\Delta \bar{q}(x)] \propto\left(x \ln ^{2} x\right)^{-1}$. This unusually rapid growth at small $x$ is used to produce the large polarized sea quark contribution.

However, this approach seems to us highly speculative and is governed by the desire to obtain $\Delta \Sigma \simeq 0$. We have hopefully convinced the reader that the EMC experiment does not demand $\Delta \Sigma \simeq 0$ and that, moreover, the Generalized Goldberger-Treiman relation is nicely consistent with the intuitive belief that the value of $\Delta \Sigma$ is not far from 1 .

Finally we comment upon an attempt to resolve the issue by means of a lattice calculation [MAN 90] of the anomalous gluon contribution to $a_{0}$. The idea is to try to measure $\Delta g$ by measuring $\left\langle P, \lambda\left|\widetilde{K}_{\mu}\right| P, \lambda\right\rangle$, or something related to it, on a lattice. The choice of lattice variables to represent $\widetilde{K}_{\mu}$ is a non-trivial task but seems to have been successfully carried out. The simulation is done on a $6^{3} \times 10$ lattice at $\beta=6 / g^{2}=5.7$, thus corresponding to $\alpha_{s} \simeq 0.08$.

In [MAN 90] Eq. (6.4.8) was used to extract $\Delta g$, which is found to be small compared with what is needed to explain the EMC experiment with a reasonably large $\Delta \Sigma$. Namely it is claimed that $|\Delta g| \lesssim 0.5$.

However, we know from Section 9.4 that (6.4.8) is not the whole story, and the relation is modified by the ghost contribution. Indeed from (9.4.1) and (9.4.3) we see that the matrix element of $\widetilde{K}_{\mu}$ in the forward direction is quite ambiguous - the limit $q^{2} \rightarrow 0$ must be carefully specified. In fact two different matrix elements were studied, namely

$$
\lim _{P \rightarrow 0}\left\langle\mathbf{0}, \boldsymbol{S}^{\prime}|\widetilde{\boldsymbol{K}} \cdot \boldsymbol{S}| \boldsymbol{P}, \boldsymbol{S}\right\rangle \quad \text { and } \quad \lim _{P \rightarrow 0} \frac{\boldsymbol{S} \cdot \hat{\boldsymbol{P}}}{P}\left\langle\mathbf{0}, \boldsymbol{S}^{\prime}\left|\partial_{\mu} \widetilde{K}^{\mu}\right| \boldsymbol{P}, \boldsymbol{S}\right\rangle
$$

where $\hat{\boldsymbol{P}}$ is a unit vector along $\boldsymbol{P}$ and $P=|\boldsymbol{P}|$.

Using (9.4.1) and (9.5.4) the values found for these matrix elements lead to the bounds

$$
\begin{aligned}
\left|-\frac{3 \alpha_{s} \Delta g}{2 \pi}+\frac{1}{3} \frac{\sqrt{3} f_{0} g_{\eta_{0} N \bar{N}}}{2 M}\right| & \lesssim \frac{1}{20} \\
\left|-\frac{3 \alpha_{s} \Delta g}{2 \pi}+\frac{\sqrt{3} f_{0} g_{\eta_{0} N \bar{N}}}{2 M}\right| & \lesssim \frac{5}{20} .
\end{aligned}
$$

We see that the smallness of the numerical results does not imply that $\Delta g$ is small. Moreover the smallness of (9.5.9) just confirms the result (9.5.5) which was obtained from the Generalized Goldberger-Treiman relation and is thus in complete harmony with the interpretation of the EMC result where a large $\Delta \Sigma$ is cancelled by a significant gluonic contribution! Similar comments apply to a more recent numerical study [ALT 94a].

However, there is another difficulty with the interpretation of the lattice result [EFR 92; MAN 92]. The calculation is done in the so-called quenched approximation which means that closed $q \bar{q}$ loops are not taken into account. It is easy to see diagramatically that this implies that any $\eta^{\prime}$ propagator in the lattice calculation of the matrix elements (9.5.7) is effectively a bare propagator i.e. the $\eta^{\prime}$ mass is not shifted from the value $m_{0}$. There will thus be a contribution to (9.5.7) proportional to the bare $\eta^{\prime}$ propagator i.e. proportional to $\left(m_{0}^{2}-q^{2}\right)^{-1}$. Bearing in mind that $m_{0}$ vanishes in the chiral limit $m_{q} \rightarrow 0$ we see that 
there should be a singularity in the quenched lattice calculation of (9.5.7) in the chiral limit as $q^{2} \rightarrow 0$. This suggests that the numerical results should be treated with caution until the quenched approximation can be overcome.

It is interesting to note that an analogous problem would arise in a quenched lattice calculation of matrix elements of $\partial^{\mu} J_{5 \mu}^{0}$, which is central to the Adler-Bardeen relation. In this case the order of taking the limits $q^{2} \rightarrow 0, m_{q}^{2} \rightarrow 0$ determines whether or not a singularity should occur. Thus, in putting $q^{2}=0$ and then taking the limit $m_{q}^{2} \rightarrow 0$ one finds a cancellation of the singularity between the matrix elements of $P$ [see (9.3.3)] and the anomaly. This was not taken into account in the lattice calculations. However, taking $m_{q}^{2}=0$ and then latting $q^{2} \rightarrow 0$ produces a singularity in $q^{2} G_{2}\left(q^{2}\right)$ of Eq. (9.4.14).

\section{$10 \boldsymbol{g}_{\mathbf{1 , 2}}(\boldsymbol{x})$ in the QCD Field Theoretic Model}

In Section 3.2 and 3.3 we discussed various attempts to calculate $g_{2}(x)$ in the Naive Parton Model and in more sophisticated variants thereof. We emphasized that these calculations do not lead to coherent results. Different authors obtain different results and these are usually incompatible with each other. The basic origin of this difficulty was exposed in Section 3.4 where we explained that $g_{2}(x)$ simply cannot be calculated within the framework of a Parton Model.

In this Section we attack the problem from the much more solid basis of the twist3 QCD Field Theoretic Model. We calculate both $g_{1}(x)$ and $g_{2}(x)$ so as to be able to emphasize the differences between the two calculations.

The first 'rigorous' derivation of the result for $g_{2}(x)$ is due to Efremov and Teryaev [EFR 84]. It is closely connected with the problem of single spin asymmetries in QCD [HEL 91]. This work was somewhat extended by Ratcliffe [RAT 86] and by Efremov and Teryaev in a later paper [EFR 87; QIU 91].

\subsection{The QCD Field Theoretic Model}

Let us now turn to the QCD Field Theoretic Model. In this case one deals with a Feynman diagram one part of which is handled perturbatively and the other, being essentially non-perturbative, is parametrized in terms of a small number of unknown functions which play the rôle of the various parton number densities.

In the simplest diagram only quarks of 4-momentum $k$ from the hadron are involved and the diagram is split into a 'hard' part $E_{\alpha \beta}^{\mu \nu}$ and a 'soft' part $\Phi_{\alpha \beta}$ as shown in Fig. 10.1.

Both $E$ and $\Phi$ are absorptive parts for the reactions $\gamma^{*} q \rightarrow X$ and hadron $\rightarrow q X$ respectively. There are no explicit propagators for the quark lines; they are absorbed into $\Phi$.

The mathematical structure which represents the contribution of Fig. 10.1 to $W^{\mu \nu}$ is

$$
W^{\mu \nu}=\frac{1}{2 \pi} \int \frac{d^{4} k}{(2 \pi)^{4}} E_{\beta \alpha}^{\mu \nu}(q, k) \Phi_{\alpha \beta}(P, S ; k)
$$

where

$$
\Phi_{\alpha \beta}=\int d^{4} z e^{i k \cdot z}\left\langle P, S\left|\bar{\psi}_{\beta}(0) \psi_{\alpha}(z)\right| P, S\right\rangle
$$

The label $\alpha$ on $\psi_{\alpha}(z)$ is a spinor label. $\psi_{\alpha}(z)$ is a column vector in colour space, so $\bar{\psi} \psi$ involves a sum over colour. Colour is irrelevant in $E^{\mu \nu}$. 
Using matrix notation for the spinor indices, (10.1.1) becomes

$$
W^{\mu \nu}=\frac{1}{2 \pi} \int \frac{d^{4} k}{(2 \pi)^{4}} \operatorname{Tr}\left[E^{\mu \nu}(q, k) \Phi(P, S ; k)\right] .
$$

The Parton Model usually emerges upon making the following approximation:

1) The $\gamma^{*} q$ interaction is given its simplest form, as shown in Fig. 10.2.

In the following we calculate only with the uncrossed Born diagram. The result for the crossed diagram is obtained at the end by the replacement $x_{B j} \rightarrow-x_{B j}$ in the hadronic matrix elements connected with $\Phi_{\alpha \beta}$ and is simply to be added to the uncrossed result. For a quark of flavour $f$, charge $e_{f}$ (in units of $e$ ), for the diagram of Fig. 10.2a,

$$
E^{\mu \nu}=-e_{f}^{2} \pi \gamma^{\nu}\left[\not k+\not h+m_{q}\right] \gamma^{\mu} \delta\left[(k+q)^{2}-m_{q}^{2}\right]
$$

where $m_{q}$ is the quark mass. Using Fig. 10.2 in Fig. 10.1 gives rise to the familiar 'handbag diagram'. In order to isolate the antisymmetric part of $W^{\mu \nu}$ we make the replacement in (10.1.4):

$$
\begin{gathered}
\gamma^{\nu} \gamma^{\rho} \gamma^{\mu} \rightarrow \frac{1}{2}\left[\gamma^{\nu} \gamma^{\rho} \gamma^{\mu}-\gamma^{\mu} \gamma^{\rho} \gamma^{\nu}\right]=-i \varepsilon^{\mu \nu \rho \sigma} \gamma_{\sigma} \gamma_{5} \\
\gamma^{\nu} \gamma^{\mu} \rightarrow \frac{1}{2}\left[\gamma^{\nu} \gamma^{\mu}-\gamma^{\mu} \gamma^{\nu}\right]=i \sigma^{\mu v}
\end{gathered}
$$

and recalling (2.1.8) find

$$
\begin{aligned}
W_{\mu \nu}^{(A)} & =\frac{e_{f}^{2}}{2} \int \frac{d^{4} k}{(2 \pi)^{4}} \delta\left[(k+q)^{2}-m_{q}^{2}\right]\left\{\varepsilon_{\mu \nu \rho \sigma}\left(q^{\rho}+k^{\rho}\right) \operatorname{Tr}\left[\gamma^{\sigma} \gamma_{5} \Phi\right]\right. \\
& \left.-m_{q} \operatorname{Tr}\left[\sigma_{\mu \nu} \Phi\right]\right\} .
\end{aligned}
$$

2) One assumes that the soft matrix element cuts off rapidly for $k^{2}$ off the mass-shell $k^{2}=m_{q}^{2}$, and for $k^{\mu}$ non-collinear with respect to the hadron momentum $P^{\mu}$.

This is implemented as follows. Consider a reference frame where the hadron is moving at high momentum along $O Z$ so that

$$
P^{\mu}=(E, 0,0, P) \quad \text { with } \quad E \approx P
$$

is a 'large' four-vector. We introduce a 'small' null vector

$$
n^{\mu}=\left(\frac{1}{P+E}, 0,0,-\frac{1}{P+E}\right)
$$

such that

$$
n \cdot P=1, \quad n^{2}=0 .
$$

One can then write for $k^{\mu}$

$$
k^{\mu}=(k \cdot n) P^{\mu}+\frac{1}{2}\left[\frac{k^{2}+\boldsymbol{k}_{T}^{2}}{(k \cdot n)}-M^{2}(k \cdot n)\right] n^{\mu}+k_{T}^{\mu}
$$

where

$$
k_{T}^{\mu}=\left(0, \boldsymbol{k}_{T}, 0\right) .
$$

In view of the assumption about $\Phi$ we can say that

$$
k^{\mu} \approx(k \cdot n) P^{\mu} .
$$

It should be noted that some care is necessary in deciding whether the approximation (10.1.13) is adequate. We shall see that this depends crucially upon whether we are considering a nucleon with longitudinal $(L)$ or with transverse $(T)$ polarization. 


\section{$10.2 g_{1}(x)$ : Longitudinal polarization}

For the study of $g_{1}$ we consider a nucleon with helicity $\lambda= \pm 1 / 2$ and it is sufficient to approximate (10.1.7) by putting

$$
(q+k)^{\rho} \approx q^{\rho}+(k \cdot n) P^{\rho}
$$

and dropping the term proportional to the quark mass $m_{q}$. Then writing

$$
q^{\rho}+(k \cdot n) P^{\rho}=\int d x \delta(x-k \cdot n)[q+x P]^{\rho}
$$

we can take the integration over $d^{4} k$ in (10.1.7) through to obtain

$$
W_{\mu \nu}^{(A)}=\frac{e_{f}^{2}}{2} \varepsilon_{\mu \nu \rho \sigma} \int d x \frac{\delta\left(x-x_{\mathrm{Bj}}\right)}{2 P \cdot q}(q+x P)^{\rho} A^{\sigma}(x)
$$

where (using $S_{L}$ to denote longitudinal spin)

$$
\begin{aligned}
A^{\sigma}(x) & \equiv \int \frac{d^{4} k}{(2 \pi)^{4}} d^{4} z \delta(x-k \cdot n) e^{i k \cdot z}\left\langle P, S_{L}\left|\bar{\psi}(0) \gamma^{\sigma} \gamma_{5} \psi(z)\right| P, S_{L}\right\rangle \\
& =\int \frac{d \lambda}{2 \pi} e^{i \lambda x}\left\langle P, S_{L}\left|\bar{\psi}(0) \gamma^{\sigma} \gamma_{5} \psi(\lambda n)\right| P, S_{L}\right\rangle
\end{aligned}
$$

is a pseudo-vector which can only depend upon the vectors $P^{\mu}, n^{\mu}$ and $v^{\mu} \equiv \varepsilon^{\mu \alpha \beta \gamma} S_{\alpha} P_{\beta} n_{\gamma}$ and the pseudo-vector $S^{\mu}$, and which must be linear in $S^{\mu}$. Given that $S \cdot P=0$ the only possibilities are $S^{\sigma}$ and $(n \cdot S) P^{\sigma}$. Note that with the normalization

$$
\left\langle\boldsymbol{P} \mid \boldsymbol{P}^{\prime}\right\rangle=(2 \pi)^{3} 2 E \delta^{3}\left(\boldsymbol{P}-\boldsymbol{P}^{\prime}\right)
$$

$A^{\sigma}(x)$ has dimensions $[M]$.

Recall that for a nucleon with 4-momentum given by (10.1.8)

$$
S^{\mu}(\lambda)=\frac{2 \lambda}{M}(P, 0,0, E) \quad S^{2}=-1
$$

where $\lambda$ is a helicity $\left(\lambda= \pm \frac{1}{2}\right)$ so that

$$
S^{\mu}(\lambda)=\frac{2 \lambda}{M}\left(P^{\mu}-M^{2} n^{\mu}\right)
$$

and we may take

$$
S^{\mu}(\lambda) \approx \frac{2 \lambda}{M} P^{\mu}
$$

i.e. $M S^{\mu}(\lambda)$ is a 'large' vector.

In view of (10.2.8) the structures $(n \cdot S) P^{\sigma}$ and $S^{\sigma}(\lambda)$ are equivalent in leading order and the only possibility is then (the factor 4 is for later convenience)

$$
A^{\sigma}(x, \lambda)=4 M h_{L}(x) S^{\sigma}(\lambda)
$$

where the dimensionless longitudinal distribution function is given by

$$
4 h_{L}(x)=\frac{n_{\sigma} A^{\sigma}(x)}{2 \lambda}=\int \frac{d \tau}{2 \pi} e^{i \tau x} \widetilde{h}_{L}(\tau)
$$

1) There appears to be a sign error in the definition of $h_{L}(x)$ in [COR 92]. Our $h_{L}(x)=-\frac{1}{2} h_{L}^{C O R}$, the factor $1 / 2$ being included so as to obtain (10.2.14). Our sign agrees with [EFR 84]. 
where

$$
\widetilde{h}_{L}(\tau)=\frac{1}{M\left(n \cdot S_{L}\right)}\left\langle P, S_{L}\left|\bar{\psi}(0) \not h \gamma_{5} \psi(\tau n)\right| P, S_{L}\right\rangle .
$$

Substituting into (10.2.3) and adding the contribution from the crossed Born diagram, Fig. 10.2b, yields

$$
W_{\mu \nu}^{(A)}(L)=e_{f}^{2} \frac{M}{P \cdot q}\left[h_{L}\left(x_{\mathrm{Bj}}\right)+h_{L}\left(-x_{\mathrm{Bj}}\right)\right] \varepsilon_{\mu \nu \rho \sigma} q^{\rho} S^{\sigma}(\lambda) .
$$

Note that the term $x P^{\rho}$ in (10.2.3) does not contribute on account of (10.2.8). Consequently (10.2.12) is gauge invariant $q^{\mu} W_{\mu \nu}^{(A)}=0$. Note that (10.2.8), which holds only for longitudinal spin, is crucial for the gauge invariance.

Comparing with (2.1.19) in the approximation $S^{\mu} \propto P^{\mu}$ we obtain, for the contribution of a quark of flavour $f$,

$$
g_{1}(x)=\frac{1}{2} e_{f}^{2}\left[h_{L}^{f}(x)+h_{L}^{f}(-x)\right] .
$$

If one treats the quark fields in (10.2.10) as free fields and regards the nucleon as an assemblage of free partons one finds

$$
h_{L}^{f}(x)=\Delta q_{f}(x) \quad h_{L}^{f}(-x)=\Delta \bar{q}_{f}(x)
$$

so that (10.2.13) reproduces the simple Parton Model result for $g_{1}(x)$. Equation (10.2.13) provides a field theoretic generalization of the Parton Model result. A more intuitive expression is given in Section 10.5. We now consider how the transverse spin case differs from the above.

\section{$10.3 g_{2}(x)$ : Transverse polarization}

In order to see the essential difference between the longitudinal and transverse spin cases consider again the result (10.2.12). In the CM of the $\gamma^{*}$-nucleon collision, as far as magnitudes are concerned, for the longitudinal case we have

$$
\left|q_{\sigma}\right| \sim\left|M S_{\sigma}(\lambda)\right|=\mathcal{O}(\nu) ; \quad P \cdot q=M \nu
$$

so that for the large components of $W_{\mu \nu}^{(A)}(L)$,

$$
\left|W_{12}^{(A)}(L)\right|=\mathcal{O}(\nu / M)
$$

assuming that $\left|h_{L}(x)\right|=\mathcal{O}(1)$.

In the transverse spin case the analogue of (10.2.4) can only be proportional to $S_{T}^{\sigma}$ since $n \cdot S_{T}=0$, and will produce a result like (10.2.12) with $S(\lambda) \rightarrow S_{T}$. Given that $\left|S_{T}\right|=\mathcal{O}(1)$ one has, for the 'large' components, only

$$
\left|W_{\mu \nu}^{(A)}(T)\right|=\mathcal{O}(1) .
$$

This immediately suggests that care must be exercised in neglecting non-leading terms e.g. in (10.1.11).

Secondly, note that in $(10.2 .1)$ the term $(k \cdot n) P^{\mu}$ of $(10.1 .11)$ did not contribute because of the fact that, in leading order, $P_{\mu} \propto S_{\mu}(\lambda)$. In the transverse case this will not happen and the analogue of (10.2.12) will contain a term $\varepsilon_{\mu \nu \rho \sigma} P^{\rho} S_{T}^{\sigma}$ in $W_{\mu \nu}^{(A)}(T)$ which, (analogously to the Parton Model case (3.4.3) when $m \neq m_{q}$ ) is not gauge invariant. 
We must therefore return to (10.1.4) and improve upon the approximation (10.1.13). However, it will then turn out that the more complicated non Parton Model diagram involving gluon exchange, Fig. 10.3, contributes to the same order. This is not entirely surprising given that the operator product result (5.2.2) for the moments of $g_{2}(x)$ involves $A_{\mu}$.

Amazingly, as was shown by Efremov and Teryaev [EFR 84], this term just cancels the unwanted contribution from the $(k \cdot n) \not P$ and the mass terms of the handbag diagram and the final result is gauge invariant. Essential in this proof is the use of the equations of motion for the quark field.

The analysis to show the cancellation is rather complicated and is carried out in Appendix C. We shall only state the result here. It is the exact analogue of (10.2.12), namely, including the contribution of the crossed Born diagram Fig. 10.2b,

$$
W_{\mu \nu}^{(A)}(T)=e_{f}^{2} \frac{M}{P \cdot q}\left[f_{T}\left(x_{B j}\right)+f_{T}\left(-x_{B j}\right)\right] \varepsilon_{\mu \nu \rho \sigma} q^{\rho} S_{T}^{\sigma}
$$

where the analogue of $(10.2 .9)$ is

$$
A^{\sigma}(x, T)=4 M f_{T}(x) S_{T}^{\sigma}
$$

with ए1)

$$
4 f_{T}(x)=\int \frac{d \tau}{2 \pi} e^{i \tau x} \widetilde{f}_{T}(\tau)
$$

where

$$
\widetilde{f}_{T}(\tau)=\frac{1}{M}\left\langle P, S_{T}\left|\bar{\psi}(0) \gamma_{5} \$_{T} \psi(\tau n)\right| P, S_{T}\right\rangle
$$

Comparing (10.3.4) with (2.1.19), for the case of transverse polarization, we obtain for the contribution of a quark of flavour $f$,

$$
g_{1}(x)+g_{2}(x)=\frac{e_{f}^{2}}{2}\left[f_{T}^{f}(x)+f_{T}^{f}(-x)\right] .
$$

Although the surviving contribution comes from the 'handbag' diagram it does not, in fact, have any simple parton interpretation as is explained briefly in Section 10.4.

\subsection{The Naive Parton Model for $g_{2}(x)$ revisited}

The result (10.3.8) for $g_{1}(x)+g_{2}(x)$ together with the expression (10.3.6) for $f_{T}(x)$ which only involves quark field operators might suggest that there ought to be a formula for $g_{1}(x)+g_{2}(x)$ derivable in the Naive Parton Model. However one can easily see that this is not so.

In the Naive Parton Model we would treat $\psi(z)$ as a free field and the proton as an assemblage of free partons. The key element in evaluating (10.3.7) would then be the matrix element.

$$
\left\langle k, s^{\prime}\left|\bar{\psi}(0) \gamma_{5} \$_{T} \psi(\tau n)\right| k, s\right\rangle
$$

taken between free quark states labelled by the covariant spin vectors $s$ and $s^{\prime}$. This matrix element will be multiplied by proton wave functions giving the amplitudes for finding quarks with $(k, s)$ and $\left(k, s^{\prime}\right)$ respectively. There are essentially two possibilities:

i) If we choose to use quark states of definite helicity $\lambda, \lambda^{\prime}$, for massless quarks the matrix element (10.4.1) is diagonal i.e. $\left\langle k, \lambda^{\prime}|\ldots| k, \lambda\right\rangle \propto \delta_{\lambda^{\prime} \lambda}$ and the product of

\footnotetext{
1) $\operatorname{Our} f_{T}(x)=2 f_{T}^{\mathrm{COR}}(x)$ for reasons explained after Eqn. (10.2.10).
} 
wave-functions becomes their modulus squared, yielding a probabilistic interpretation. But in this case one finds that (10.4.1) is proportional to $m_{q} S_{T} \cdot s(\lambda)$ which is zero in the Naive Parton Model with no $k_{\perp}$. So any non-zero result is only of order $k_{\perp}$ and cannot be evaluated without a model of the $k_{T}$-distribution, an aspect of the nucleon structure which is usually considered to be beyond the Naive Parton Model.

ii) If we choose to use quark states of definite transverse spin e.g. states

$$
\mid \uparrow \text { or } \downarrow\rangle=\frac{1}{\sqrt{2}}(|+\rangle \pm i|-\rangle),
$$

then the matrix element (10.4.1) is zero for massless quarks if $s^{\prime}=s$ and is largest for non-diagonal transitions $|\uparrow\rangle \rightarrow|\downarrow\rangle$. Consequently one does not obtain a probabilistic expression from the nucleon wave-functions. Moreover even the non-diagonal transitions vanish in the absence of $k_{\perp}$.

Of course the above arguments do not mean that one cannot do model calculations of $g_{1}(x)+g_{2}(x)$. They simply imply that the results will not have the traditional Parton Model form and will depend upon specific assumptions about the $k_{\perp}$ dependence of the nucleon wave-function.

\subsection{Probabilistic form for $g_{1}(x)$ in the Field Theoretic Model}

We mentioned in Section 10.2 that the hadronic matrix element $h_{L}(x)$, in terms of which $g_{1}(x)$ is given in (10.2.13), reduces to the usual partonic $\Delta q(x)$ when the fields are treated as non-interacting free fields. However even in the case of interacting fields one can rewrite the formulae $(10.2 .10,11)$ for $h_{L}(x)$ in such a way as to display manifestly the probabilistic interpretation.

From the analysis of the matrix element $\left\langle P, S_{L}\left|\bar{\psi}(0) \gamma^{\mu} \gamma_{5} \psi(\tau n)\right| P, S_{L}\right\rangle$ given in Eq. (E.1) it is easy to see that to leading order the matrix elements with $\mu=0$ and $\mu=3$ are equal. From the definition of $n^{\mu}(10.1 .9)$ we can thus replace the factor $\not h$ which occurs in the definition of $h_{L}(x)$ by

$$
\not h=\frac{1}{P+E}\left(\gamma^{0}+\gamma^{3}\right) \rightarrow \frac{2}{P+E} \gamma^{0} \approx \frac{\gamma^{0}}{P} .
$$

Inside the matrix element (10.2.11) we may then write

$$
\begin{aligned}
\bar{\psi}(0) h \gamma_{5} \psi(\tau n) & \rightarrow \frac{1}{P} \bar{\psi}(0) \gamma^{0} \gamma_{5} \psi(\tau n)=\frac{1}{P} \psi^{\dagger}(0) \gamma_{5} \psi(\tau n) \\
& =\frac{1}{P}\left\{\psi^{\dagger}(0) \frac{1+\gamma_{5}}{2} \psi(\tau n)-\psi^{\dagger}(0) \frac{1-\gamma_{5}}{2} \psi(\tau n)\right\} \\
& =\frac{1}{P}\left\{\left(\frac{1+\gamma_{5}}{2} \psi(0)\right)^{\dagger}\left(\frac{1+\gamma_{5}}{2} \psi(\tau n)\right)\right. \\
& \left.-\left(\frac{1-\gamma_{5}}{2} \psi(0)\right)^{\dagger}\left(\frac{1-\gamma_{5}}{2} \psi(\tau n)\right)\right\}
\end{aligned}
$$

where, in the last step, we have used

$$
\left(\frac{1 \pm \gamma_{5}}{2}\right)^{2}=\left(\frac{1 \pm \gamma_{5}}{2}\right)
$$

Finally, inserting a sum over a complete set of states, using translational invariance to shift from $\psi(\tau n)$ to $\psi(0)$, and carrying out the integration over $\tau$ in (10.2.10), we 
obtain, taking a nucleon with helicity $\lambda=+1 / 2$,

$$
h_{L}(x)=q_{+}(x)-q_{-}(x)
$$

where

$$
q_{ \pm}(x) \equiv \frac{1}{4 P} \sum_{X}\left|\left\langle X\left|\frac{1 \pm \gamma_{5}}{2} \psi(0)\right| P, \lambda=1 / 2\right\rangle\right|^{2} \delta\left[n \cdot P_{X}-(1-x)\right] .
$$

This displays manifestly the probabilistic nature of $q_{ \pm}(x)$ even in the fully interactive theory.

The same approach clearly fails for $f_{T}(x)$ given by $(10.3 .6,7)$, in terms of which $g_{2}(x)$ is given by $(10.3 .8)$.

Concerning our expression (10.5.5) for $q_{ \pm}(x)$, one can derive a more accurate result, in which one does not make the approximation that the matrix elements with $\mu=0$ and $\mu=3$ are equal, using the formalism of light-cone fields [MAN 91a].

Define

$$
\gamma^{ \pm}=\frac{1}{\sqrt{2}}\left(\gamma^{0} \pm \gamma^{3}\right)
$$

and projection operator

$$
P_{+}=\frac{1}{2} \gamma^{-} \gamma^{+} \quad P_{+}^{2}=P_{+}
$$

and "good" component of the field

$$
\psi_{+} \equiv P_{+} \psi
$$

Then a more accurate expression for $q_{ \pm}(x)$ is

$$
q_{ \pm}(x)=\frac{1}{4 P} \sum_{X}\left|\left\langle X\left|\frac{1 \pm \gamma_{5}}{2} \psi_{+}(0)\right| P, \lambda=1 / 2\right\rangle\right|^{2} \delta\left[n \cdot P_{X}-(1-x)\right] .
$$

\section{Future experiments}

Throughout this paper we have stressed the rôle of the axial anomaly in providing an unexpected gluon contribution to the first moments $\Gamma_{1}^{p, n}$ measured in polarized deep inelastic lepton-hadron scattering. As a consequence of this mechanism the data can be explained with a large spin contribution $\Delta \Sigma \simeq 0.7$ from the quarks, as expected intuitively. This is in accord with the Generalized Goldberger-Treiman relation and with various relativistic bag model calculations. The smallness of the flavour singlet part of $\Gamma_{1}^{p}$ is explained as a cancellation between $\Delta \Sigma$ and the anomalous gluon contribution.

However, in order to accomplish this one requires a large gluon spin $\Delta g$ at $\left\langle Q^{2}\right\rangle \simeq 10$ $(\mathrm{GeV} / \mathrm{c})^{2}$, i.e. $\Delta g \simeq 4$. As we have explained, it is inevitable, within QCD-evolution, that $\Delta g$ grows indefinitely with $Q^{2}$, so even if intuitively we expect $\Delta g$ very small at small $Q^{2}$ we really cannot rule out the above value at $\left\langle Q^{2}\right\rangle \simeq 10(\mathrm{GeV} / \mathrm{c})^{2}$.

But, clearly, it is of the utmost importance to test this explanation by measuring $\Delta g$ in other, independent reactions. If it is as large as the DIS experiments suggest then there will be measurable effects in several other processes. In the following we indicate briefly some of the possibilities that have been studied thus far [WIN 92, REY 93].

In Section 7.3 we explained how the growth of $\Delta g$ is compensated for by an analogous growth in $L_{z}$, the total orbital angular momentum carried by all partons. The hard processes we are about to discuss, being short range interactions, are insensitive to $L_{z}$ and can thus reveal $\Delta g$ directly. 


\subsection{Semi-inclusive deep inelastic lepton-hadron reactions}

Perhaps the most straightforward proposal [CAR 88, MAN 91] is in semi-inclusive polarized DIS.The idea is to select events with final state hadrons of large $p_{T}$ relative to the $\gamma^{*}$-nucleon collision axis. Such events should be enriched by two-jet production via the photon-gluon fusion subprocess shown in Fig. 8.2 and will depend directly on $\Delta g$. The contribution to $\Gamma_{1}^{p}$ coming from this subclass of events should be sensitive to $\Delta g$ and could even be negative for some choice of $p_{T}$ cut-off.

Very much in the same spirit one could select on events with charm pair production [GLU 88] or on $J / \psi$ production at large $p_{T}$ [KAL 89; SRI 92]. The mechanism is the same as shown in Fig. 8.2. These processes are quite sensitive to $\Delta g$.

Another proposal is to look at the semi-inclusive production of forward kaons, which should be sensitive to the strange quarks in the nucleon [CLO 89]. The idea here is to measure the polarization of the strange quarks in the nucleon, i.e. $\Delta s$. This is important because if the anomalous gluon contribution is NOT the correct mechanism to explain the smallness of the flavour singlet part of $\Gamma_{1}^{p}$ then one can arrange to have $\Delta \Sigma \simeq 0$ by invoking a surprisingly large and negative polarized strange quark distribution, as was discussed in Section 4.3.3. However the interpretation of the experiment might be difficult because some contribution could arise from $s \bar{s}$ pairs created in the photon-gluon fusion diagram, Fig. 8.2.

Most of the above measurements should be feasible in the present SMC (CERN) and SLAC experiments (provided high enough statistics and good enough particle identification can be achieved) and in the next generation of experiments now being planned, viz. HERMES at DESY and HELP at CERN.

\subsection{Hadron-hadron reactions}

If it is possible to produce high energy collisions of longitudinally polarized protons or antiprotons on a longitudinally polarized nucleon target then a whole range of experimental possibilities become feasible for studying $\Delta g$.

\subsubsection{Hard $\boldsymbol{\gamma}$ and Drell-Yan reactions}

Several possibilities have been analysed (see [BER 89]). The classic and most direct experiment would be the polarization asymmetry in the production cross-section for hard $\gamma^{\prime}$ s at high $p_{T}$. The obvious asymmetry

$$
A=\frac{d \sigma^{\overrightarrow{3}}-d \sigma^{\rightleftarrows}}{d \sigma^{\vec{\rightarrow}}+d \sigma^{\rightleftarrows}}
$$

depends strongly on $\Delta g$ and $\Delta q$.

It should also be possible to detect the polarization asymmetry in the production cross-section for a high $p_{T}$ jet [RAM 88], but this will be less sensitive to $\Delta g$ than hard $\gamma$ production because quark initiated processes play a relatively larger rôle.

Experiments of the above type are planned for the RHIC collider now under construction at Brookhaven but it might also be feasible to do such measurements using the secondary polarized proton beam colliding with a fixed polarized target at the Fermilab TEVATRON. 
One of the most interesting possibilities for learning about $\Delta g$ in hadron-hadron collisions involves the production of Drell-Yan lepton pairs at large $p_{T}$, i.e. where the $p_{T}$ of the pair is large with respect to the collision axis. Of course the obvious experiment, analogous to the hard $\gamma$ case, is the production asymmetry using both polarized beam and polarized target. But what is especially interesting about the Drell-Yan case, given the difficulty of providing both a polarized beam and a polarized target at high energies, is that certain asymmetries sensitive to $\Delta g$ exist even when just the beam or just the target is longitudinally polarized.

The problem is that these single spin asymmetries vanish in the usual Born mechanism for large $p_{T}$ production shown in Fig. 11.1. A non-zero asymmetry arises from $\gamma-Z^{0}$ interference for large mass pairs [LEA 93, 94a] or from interference between the Born diagrams and the one loop QCD corrections to $q g \rightarrow q \gamma$ and $q \bar{q} \rightarrow \gamma g$ [CAR 92].

The most complete information is obtained if the angular distribution of the lepton in the pair rest frame can be measured [LEA 93, 94a]. Alternatively, one can measure what might be called the handedness $(H)$ of the lepton pair. Let $\boldsymbol{k}_{ \pm}$be the laboratory momentum of the $\ell^{ \pm}$and $\boldsymbol{P}$ be the laboratory momentum of the proton polarized beam (or target). Define

$$
X_{l} \equiv \boldsymbol{P} \cdot\left(\boldsymbol{k}_{+} \times \boldsymbol{k}_{-}\right)
$$

and let $N\left(X_{l}\right)$ be the number of pairs with given value of $X_{l}$. Then

$$
H \equiv \frac{N\left(X_{l}>0\right)-N\left(X_{l}<0\right)}{N\left(X_{l}>0\right)+N\left(X_{l}<0\right)}
$$

is sensitive to $\Delta g$.

All such single spin experiments, however, look difficult because of the small crosssections. It has also been pointed out that there is a correlation between the polarization of the produced lepton or direct photon and the polarization of the beam [CON 91], which depends on $\Delta g$. Such an experiment might be feasible with muon pairs, but clearly it is very difficult to monitor the final lepton polarization.

\subsubsection{Heavy quark production}

Probably the most sensitive experiment to $\Delta g$ and, in many ways, perhaps the most realistic, is the polarization asymmetry in the production of heavy quarks using longitudinally polarized beam and target [COR 88]. In this case the dominant subprocess is the gluon fusion reaction $g+g \rightarrow q+\bar{q}$ and the asymmetry will be proportional to $(\Delta g)^{2}$. Sizable asymmetries are expected and should be measurable at RHIC.

\subsection{Jet handedness}

If it were possible to measure the polarization of the high $p_{T}$ quark produced in the partonic reaction

$$
q+\vec{g} \rightarrow \vec{q}+g
$$

where an unpolarized quark collides with a polarized gluon, then one would have a very direct measure of the gluon polarization. The advantage of such an approach is that it requires only an unpolarized beam colliding with a polarized target. The problem is to

measure the final quark's polarization in the dominant process where it hadronizes into a jet. Thus one has to study the reaction

$$
p+\vec{p} \rightarrow j e t+X
$$


and somehow monitor the polarization of the quark which initiated the jet [STR 92]. (One is effectively trying to measure the longitudinal spin transfer $D_{L L}$ [BOU 80].)

One can define the handedness of a jet and show that it is proportional to the polarization of the initiating parton [NAC 77; EFR 78, 92a]. Namely, analogously to (11.2.2) one can define

$$
X_{L} \equiv \frac{\boldsymbol{P}_{J} \cdot\left(\boldsymbol{p}_{1} \times \boldsymbol{p}_{2}\right)}{\left|\mathbf{P}_{J}\right|}
$$

where $\boldsymbol{P}_{J}$ is the jet momentum and $\boldsymbol{p}_{1,2}$ are the momenta of two particles in the jet chosen according to some definite criteria. For example we could choose the leading and the second leading particles in the jet.

The handedness is then defined in terms of the number of jets with $X_{L}>0$ and $X_{L}<0$, i.e.

$$
H_{j e t}=\frac{N\left(X_{L}>0\right)-N\left(X_{L}<0\right)}{N\left(X_{L}>0\right)+N\left(X_{L}<0\right)} .
$$

(An analogous quantity can be defined based on using the momenta of the three most leading particles in the jet).

The essential point is that

$$
H_{j e t}=\alpha_{J} P_{q}
$$

where $P_{q}$ is the degree of longitudinal polarization of the initiating quark and $\alpha_{J}$ is the 'jet analysing power', the calculation of which lies in the realm of non-perturbative QCD. Thus the key issue is the size of $\alpha_{J}$.

Miraculously $\alpha_{J}$ can be measured! The point is that in the electroweak process

$$
e^{+} e^{-} \rightarrow Z^{0} \rightarrow q \bar{q} \rightarrow 2 \text { jets }
$$

the $q$ and $\bar{q}$ are significantly polarized and their polarization is known with some confidence from electroweak theory. Thus by measuring $H_{\text {jet }}$ in (11.3.5) we effectively measure $\alpha_{J}$. One can argue $\mathbb{T}$ that it is also possible to measure $\alpha_{J}$ independently of electroweak theory by studying the correlations between the handeness of the two jets in (11.3.6).

There is a theoretical estimate that $\alpha_{J} \simeq(5-10) \%$ [RYS 93, EFR 94]. If this is confirmed by measurements of (11.3.5) then it will open up the real possibility of measuring $\Delta g$ at presently existing accelerators, i.e at the Fermilab TEVATRON, the CERN SPS and the Serpukhov UNK.

\section{Conclusions}

Polarized Deep Inelastic Scattering has proved to be an exciting and controversial field in which unexpected experimental results have engendered the discovery of unexpectedly subtle theoretical problems.

Much new data has become available in the past years from the SMC at CERN and from the E142 at SLAC, including, for the first time, results on the neutron. And the controversial EMC proton data of 1987 have been confirmed by the SMC.

The principal points that have emerged are:

1. The Bjorken sum rule has been tested, and provided sufficient care is exercised in dealing with experiments at different mean $Q^{2}$ and in incorporating perturbative QCD corrections, there is absolutely no evidence for any violation of the sum rule. Earlier claims to the contrary were simply too cavalier in handling the data.

1) See [NAC 77]: note that $\alpha_{J}$ corresponds to the quantity $\frac{L-R}{L+R}$ in that paper. 
2. The measurement of the first moment $\Gamma_{1}^{p}$ of $g_{1}^{p}(x)$ does not measure the contribution of the quark spins to the spin of the proton. There is an anomalous gluon contribution which significantly alters the theoretical expression for $\Gamma_{1}^{p}$ so that the smallness of $\Gamma_{1}^{p}$ no longer implies an almost zero contribution from the quark spins. This explanation requires a significant polarized gluon density and this feature must be tested independently. The polarized gluon number density $\Delta g$ can be measured in polarized semi-inclusive deep inelastic lepton-hadron interactions, and in hadron-hadron collisions involving polarized beam and target. Both hard $\gamma$ and Drell-Yan reactions are sensitive to $\Delta g$, as is the polarization asymmetry in heavy quark production. Another possible approach is via "jet handedness". RHIC seems to be an ideal machine for exploring the property of $\Delta g$.

3. While $\Gamma_{1}^{p}$ itself no longer determines the contribution of the quark spins to the spin of the nucleon, there does exist a non-perturbative Generalized Goldberger-Treiman relation which connects the quark spin to the coupling of the $\eta^{\prime}$ to nucleons. This coupling can in principle be deduced from a detailed phase-shift analysis of elastic nucleon-nucleon scattering or from some other experiments. Estimates already exist but a more accurate determination would be of great interest.

4. It turns out that $g_{2}(x)$ cannot be calculated in the Naive Parton Model nor in the QCD Improved Parton Model. Nonetheless an expression can be obtained for it in a field theoretic approach. The result does not have a partonic interpretation.

5. Neither the Burkhardt-Cottingham nor the Efremov-Leader-Teryaev sum rules follow from the Operator Product Expansion. Indeed if simple Regge arguments can be trusted, the behaviour at small $x$ of $g_{2}$ should lead to divergent integrals in these sum rules. But the argument is not watertight and every effort should be made to test the sum rules experimentally 1 .

\section{Appendix A - Some kinematic relations amongst asymmetries and scaling functions}

The spin-spin asymmetries $A_{\|}$and $A_{\perp}$, Eqs. (2.1.27) and (2.1.38) respectively, are usually expressed in terms of virtual Compton scattering asymmetries $A_{1,2}$, as

$$
A_{\|}=D\left(A_{1}+\eta A_{2}\right)
$$

and

$$
A_{\perp}=d\left(A_{2}-\xi A_{1}\right)
$$

where

$$
\begin{gathered}
A_{1}=\frac{M \nu G_{1}-Q^{2} G_{2}}{W_{1}}=\frac{g_{1}-\left(4 M^{2} x^{2} / Q^{2}\right) g_{2}}{F_{1}} \\
A_{2}=\sqrt{Q^{2}} \frac{M G_{1}+\nu G_{2}}{W_{1}}=\frac{2 M x}{\sqrt{Q^{2}}} \frac{g_{1}+g_{2}}{F_{1}} \\
D=\frac{E-\epsilon E^{\prime}}{E(1+\epsilon R)} \quad \eta=\frac{\epsilon \sqrt{Q^{2}}}{E-\epsilon E^{\prime}} \\
d=D \sqrt{\frac{2 \epsilon}{1+\epsilon}} \quad \xi=\eta \frac{1+\epsilon}{2 \epsilon}
\end{gathered}
$$

1) Note added in proof: The first few data on $g_{2}^{p}(x)$ which have just appeared [ADA 94a] have large errors and do not allow yet a significant test of the sum rules. 
with

$$
\frac{1}{\epsilon}=1+2\left(1+\frac{\nu^{2}}{Q^{2}}\right) \operatorname{tg}^{2} \frac{\theta}{2}
$$

and, as in Eq. (2.1.32),

$$
R=\frac{W_{2}}{W_{1}}\left(1+\frac{\nu^{2}}{Q^{2}}\right)-1=\frac{F_{2}}{2 x F_{1}}\left(1+\frac{4 M^{2} x^{2}}{Q^{2}}\right)-1 .
$$

The analysis of $A_{\|}$proceeds through subsequent approximations. One first notices that both the coefficient $\eta$ and $A_{2}$, which is bounded by [LEA 85]

$$
\left|A_{2}\right| \leq \sqrt{R}
$$

are small, so that it is reasonably accurate to write

$$
A_{\|} \approx D A_{1}
$$

One then neglects the $g_{2}$ term in $A_{1}$, Eq. (A.3), and replaces $F_{1}(x)$ by

$$
F_{1}(x)=\frac{F_{2}(x)}{2 x[1+R(x)]}
$$

which originates from Eq. (A.7) for $4 M^{2} x^{2} \ll Q^{2}$.

Thus one obtains

$$
g_{1}(x) \approx \frac{A_{\|}}{D} \frac{F_{2}(x)}{2 x[1+R(x)]},
$$

which expresses $g_{1}(x)$ in terms of the measured asymmetry $A_{\|}$, the unpolarized structure function $F_{2}(x) /[2 x(1+R)]$ (also measured) and the known coefficient D. It can be shown that all the above approximations are actually quite harmless [LEA 88].

The analysis of $A_{\perp}$ is dicussed in Section 2.1.3.

\section{Appendix B - Current matrix elements in the Parton Model}

In the Standard Model all hadronic currents are expressed in terms of quark fields $\psi_{f}(x)$ where $f$ labels the flavour. We require the forward matrix elements of these currents taken between nucleon states. We shall use the quark-parton model to evaluate these in the frame $S^{\infty}$ where the proton moves along $O Z$ at high speed.

Consider the generic current

$$
J_{f}=\bar{\psi}_{f} \Gamma \psi_{f}
$$

where $\Gamma=\gamma^{\alpha}$ or $\gamma^{\alpha} \gamma_{5}$, evaluated at the space-time origin $x_{\mu}=0$. We shall evaluate the forward matrix elements of $J_{f}$ in a proton state with covariant spin vector $S_{\mu}$.

We insert complete sets of quark states, corresponding to partons of four-momentum $k$ and helicity $\lambda$.

Because the proton is polarized we need to introduce $n_{f}(\boldsymbol{k}, \lambda ; S) d^{3} \boldsymbol{k}$ as the number of flavour $f$ quarks with three-momentum in the range $\boldsymbol{k} \rightarrow \boldsymbol{k}+d^{3} \boldsymbol{k}$ and with helicity $\lambda$ inside a proton of four-momentum $P$ and covariant spin vector $S_{\mu}$. After some algebra one finds for the forward hadronic matrix elements,

$$
\left\langle P, S\left|J_{f}\right| P, S\right\rangle=\sum_{\lambda} \int d^{3} \boldsymbol{k} \frac{P_{0}}{\epsilon} n_{f}(\boldsymbol{k}, \lambda ; S)\left\langle k, \lambda\left|J_{f}\right| k, \lambda\right\rangle
$$


where $\epsilon$ is the parton energy and where we have utilised the fact that for fast quarks $\gamma^{\alpha}$ and $\gamma^{\alpha} \gamma^{5}$ conserve helicity.

Let us now define more specifically

$$
J_{f}^{\alpha}=\bar{\psi}_{f} \gamma^{\alpha} \psi_{f} \quad J_{f}^{5 \alpha}=\bar{\psi}_{f} \gamma^{\alpha} \gamma_{5} \psi_{f}
$$

Then treating the quark-partons as 'free' particles in $S^{\infty}$ one has

$$
\left\langle k, \lambda\left|J_{f}^{\alpha}\right| k, \lambda\right\rangle=\bar{u}_{\lambda} \gamma^{\alpha} u_{\lambda}=2 k^{\alpha}
$$

and

$$
\left\langle k, \lambda\left|J_{f}^{5 \alpha}\right| k, \lambda\right\rangle=\bar{u}_{\lambda} \gamma^{\alpha} \gamma_{5} u_{\lambda}=2 m_{f} s^{\alpha}(\lambda)
$$

where $s^{\alpha}(\lambda)$ is the parton's covariant spin vector corresponding to a helicity state $\lambda= \pm \frac{1}{2}$,

$$
s^{\alpha}(\lambda)=\frac{2 \lambda}{m_{f}}(|\boldsymbol{k}|, \epsilon \hat{\boldsymbol{k}}) .
$$

On grounds of Lorentz covariance the forward nucleonic matrix elements have a similar structure:

$$
\left\langle P, S\left|J_{f}^{\alpha}\right| P, S\right\rangle=2 v_{f} P^{\alpha}
$$

and

$$
\left\langle P, S\left|J_{f}^{5 \alpha}\right| P, S\right\rangle=2 M a_{f} S^{\alpha}
$$

where $v_{f}, a_{f}$ are numbers (Lorentz scalars) that measure the strength of the vector and axial-vector matrix elements. As explained in [BAI 82] the numbers $v_{f}$ are exactly known when the currents $J_{f}^{\alpha}$ are conserved currents.

Substituting (B.4 and 5) into (B.2) and using (B.7 and 8) one finds

$$
P^{\alpha} v_{f}=\sum_{\lambda} \int d^{3} \boldsymbol{k} \frac{P_{0}}{\epsilon} n_{f}(\boldsymbol{k}, \lambda ; S) k^{\alpha}
$$

and

$$
M S^{\alpha} a_{f}=\sum_{\lambda} \int d^{3} \boldsymbol{k} \frac{P_{0}}{\epsilon} n_{f}(\boldsymbol{k}, \lambda ; S) m_{f} s^{\alpha}(\lambda)
$$

Now taking in $S^{\infty}$

$$
k \approx\left(x^{\prime} P+\frac{m^{2}+\boldsymbol{k}_{T}^{2}}{2 x^{\prime} P}, \boldsymbol{k}_{T}, x^{\prime} P\right)
$$

and using it in (B.6), and taking

$$
S^{\alpha}(\Lambda)=\frac{2 \Lambda}{M}\left(P, 0,0,-P-\frac{M^{2}}{2 P}\right)
$$

for a proton of helicity $\Lambda= \pm \frac{1}{2}$, we find eventually, from (B.9 and 10)

$$
v_{f}=\int_{0}^{1} d x^{\prime}\left[q_{f}^{P}\left(x^{\prime}\right)+q_{f}^{A}\left(x^{\prime}\right)\right]=\int_{0}^{1} d x q_{f}(x)
$$

and

$$
a_{f}=\int_{0}^{1} d x^{\prime}\left[q_{f}^{P}\left(x^{\prime}\right)-q_{f}^{A}\left(x^{\prime}\right)\right]=\int_{0}^{1} d x \Delta q_{f}(x) .
$$

where $q_{f}^{P}(x)$ and $q_{f}^{A}(x)$ are respectively the number densities of quarks $f$ with spin parallel or antiparallel to the nucleon spin. 
In the above we considered only quarks. A similar arguments holds for antiquark constituents, where the partonic matrix elements are

$$
\begin{gathered}
\left\langle k, \lambda\left|J_{f}^{\alpha}\right| k, \lambda\right\rangle=-\bar{v}_{\lambda} \gamma^{\alpha} v_{\lambda}=-2 k^{\alpha} \\
\left\langle k, \lambda\left|J_{f}^{5 \alpha}\right| k, \lambda\right\rangle=-\bar{v}_{\lambda} \gamma^{\alpha} \gamma_{5} v_{\lambda}=2 m_{f} s^{\alpha}(\lambda) .
\end{gathered}
$$

Finally then one finds

$$
\begin{gathered}
v_{f}=\int_{0}^{1} d x\left[q_{f}(x)-\bar{q}_{f}(x)\right] \\
a_{f}=\int_{0}^{1} d x\left[\Delta q_{f}(x)+\Delta \bar{q}_{f}(x)\right] .
\end{gathered}
$$

The currents occuring in DIS are all just linear combination of the currents $J_{f}^{\alpha}$ and $J_{f}^{5 \alpha}$ so that all results quoted in Section 4.3 follow trivially from (B.7, 8, 16 and 17).

\section{Appendix C - Transverse spin: the restoration of electromagnetic gauge invariance}

We work in a reference system where the nucleon 4-momentum $P^{\mu}$ is given by (10.1.8) and where the null-vector $n^{\mu}$ is as in (10.1.9).

We shall choose the gauge $A \cdot n=0$ for simplicity. In the general treatment [EFR 84] where $E^{\mu \nu}(\ldots, k ; \ldots)$ of $(10.1 .3)$ does not refer just to the Born ('handbag') diagram, the technique is to replace $(10.2 .1)$ by

$$
(q+k)^{\rho}=q^{\rho}+(k \cdot n) P^{\rho}+\Delta k^{\rho}
$$

and to do a Taylor expansion in the small quantity $\Delta k^{\rho}$, so that (10.1.1) will involve $\left(\partial E^{\mu \nu}\right) /\left(\partial k_{\rho}\right)$ evaluated at $k^{\rho}=(q+x P)^{\rho}$.

The contribution to $W^{\mu \nu}$ from Fig. 10.3 which involves gluon exchange $(G)$ is of the form (we show explicitely the colour labels $i, j, a$ )

$$
\begin{aligned}
W_{G}^{\mu \nu}= & \frac{1}{2 \pi} \int \frac{d^{4} k_{1}}{(2 \pi)^{4}} \frac{d^{4} k_{2}}{(2 \pi)^{4}} E_{\beta \alpha}^{\mu \rho \nu}\left(k_{1}, k_{2}\right) g\left(\lambda^{a} / 2\right)_{j i} \\
& \times \int d^{4} z_{1} d^{4} z_{2} e^{i k_{1} z_{1}} e^{i\left(k_{2}-k_{1}\right) z_{2}}\left\langle P, S\left|\bar{\psi}_{\beta, j}(0) A_{\rho}^{a}\left(z_{2}\right) \psi_{\alpha, i}\left(z_{1}\right)\right| P, S\right\rangle .
\end{aligned}
$$

We absorb the colour matrix $\left(\lambda^{a} / 2\right)_{j i}$ and the factor $g$ into the hadronic matrix element to obtain

$$
W_{G}^{\mu \nu}=\frac{1}{2 \pi} \int \frac{d^{4} k_{1}}{(2 \pi)^{4}} \frac{d^{4} k_{2}}{(2 \pi)^{4}} \operatorname{Tr}\left[E^{\mu \rho \nu}\left(k_{1}, k_{2}\right) \Phi_{\rho}^{G}\left(P, S ; k_{1}, k_{2}\right)\right]
$$

where

$$
\Phi_{\rho}^{G}=\int d^{4} z_{1} d^{4} z_{2} e^{i k_{1} z_{1}} e^{i\left(k_{2}-k_{1}\right) z_{2}}\left\langle P, S\left|\bar{\psi}(0) g A_{\rho}\left(z_{2}\right) \psi\left(z_{1}\right)\right| P, S\right\rangle
$$

and where now $\psi$ is a column vector in spin space and in colour space and $A_{\rho}$ is the matrix $A_{\rho}=\left(\boldsymbol{\lambda}^{a} / 2\right) A_{\rho}^{a}$. The trace in (C.3) is only over spinor labels.

In the general treatment a key rôle is played by the Ward identity

$$
\frac{\partial E^{\mu \nu}}{\partial k_{\rho}}(q+x P)=E^{\mu \rho \nu}(q+x P, q+x P)
$$


which permits a connection between the leading order part of $W_{G}^{\mu \nu}$ and the Taylor expansion of $W^{\mu \nu}$ from the quark diagram of Fig. 10.2 . The reader is referred to [EFR 84] for the general analysis. Here we shall illustrate the phenomenon by taking the simplest case where $E^{\mu \nu}$ is given by the Born diagram in Fig. 10.2 and $E^{\mu \rho \nu}$ by the Born diagram shown in Fig. C.1. Both $E^{\mu \nu}$ and $E^{\mu \rho \nu}$ are independent of colour.

For this simplest case it is easier not to use the Taylor expansion (C.1) in (10.1.4) but to split $\not q+\not k+m_{q}$ into $\not q$, which yields the result (10.3.4), and the contribution from $\not k+m_{q}$, which we shall call $\Delta W_{\mu \nu}^{(A)}$, where

$$
\Delta W_{(A)}^{\mu \nu}=\frac{e_{f}^{2}}{2} \int \frac{d^{4} k}{(2 \pi)^{4}}\left\{\varepsilon^{\mu \nu \rho \sigma} \operatorname{Tr}\left[\gamma_{\sigma} \gamma_{5} \Phi_{\rho}\right]-m_{q} \operatorname{Tr}\left[\sigma^{\mu \nu} \Phi\right]\right\} \delta\left[(k+q)^{2}-m_{q}^{2}\right]
$$

where we have used (10.1.5) and (10.1.7) and where

$$
\Phi_{\rho}=\int d^{4} z e^{i k z}\left\langle P, S\left|\bar{\psi}(0) i \partial_{\rho} \psi(z)\right| P, S\right\rangle .
$$

To obtain (C.7) we have used the fact that $k_{\rho}$ can be obtained by differentiating $e^{i k z}$, plus the usual assumption that the hadronic matrix element vanishes for $|z| \rightarrow \infty$. Note for future reference that by translational invariance one can show that

$$
\bar{\psi}(0) i \vec{\partial}_{\rho} \psi(z) \Longleftrightarrow \bar{\psi}(0)\left(-i \overleftarrow{\partial}_{\rho}\right) \psi(z)
$$

are equivalent inside the matrix element in (C.7).

Introducing $I=\int d x \delta(x-k \cdot n)$, and using (C.8), Eq. (C.6) can eventually be written as

$$
\Delta W_{(A)}^{\mu \nu}=\frac{e_{f}^{2}}{8 P \cdot q} \int d x \delta\left(x-x_{B j}\right)\left\langle\varepsilon^{\mu \nu \rho \sigma} \gamma_{\sigma} \gamma_{5}\left(-i \vec{\partial}_{\rho}+i \overleftarrow{\partial}_{\rho}\right)-2 m_{q} \sigma^{\mu \nu}\right\rangle
$$

where we have introduced the shorthand for any $\mathrm{X}$

$$
\langle X\rangle \equiv \int \frac{d \tau}{2 \pi} e^{i \tau x}\left\langle P, S_{T}|\bar{\psi}(0) X \psi(\tau n)| P, S_{T}\right\rangle
$$

Turning now to the gluon diagram, using the Born approximation in Fig. C.1, we keep only the leading terms in the quark propagators:

$$
\begin{aligned}
q^{\rho}+k_{1}^{\rho} \approx q^{\rho}+\left(k_{1} \cdot n\right) P^{\rho} & =\int d x_{1} \delta\left(x_{1}-k_{1} \cdot n\right)\left(q^{\rho}+x_{1} P^{\rho}\right) \\
q^{\rho}+k_{2}^{\rho} \approx q^{\rho}+\left(k_{2} \cdot n\right) P^{\rho} & =\int d x_{2} \delta\left(x_{2}-k_{2} \cdot n\right)\left(q^{\rho}+x_{2} P^{\rho}\right) .
\end{aligned}
$$

After some manipulation (C.3) becomes

where, for any $X$,

$$
\begin{aligned}
W_{G}^{\mu \nu}= & \frac{e_{f}^{2}}{8 P \cdot q} \int d x_{1} d x_{2}\left\langle\left\langle\gamma^{\nu}\left(\not 1+x_{2} \not P\right) g A\left(\tau_{1} n\right)\left(\not 1+x_{1} \not P\right) \gamma^{\mu}\right\rangle\right\rangle \\
& \times \operatorname{Disc}\left[\frac{1}{\left(x_{1}-x_{\mathrm{Bj}}\right)\left(x_{2}-x_{\mathrm{Bj}}\right)}\left(\frac{i}{\pi P \cdot q}\right)\right]
\end{aligned}
$$

$$
\begin{aligned}
& \left\langle\left\langle X\left(x_{1}, x_{2} ; \tau_{1}\right)\right\rangle\right\rangle \equiv \int \frac{d \tau_{1}}{2 \pi} \frac{d \tau_{2}}{2 \pi} e^{i \tau_{2} x_{2}} e^{i \tau_{1}\left(x_{1}-x_{2}\right)} \\
& \quad \times\left\langle P, S_{T}\left|\bar{\psi}(0) X\left(x_{1}, x_{2}, \tau_{1} n\right) \psi\left(\tau_{2} n\right)\right| P, S_{T}\right\rangle .
\end{aligned}
$$


Note that if $X$ depends on only one of the variables $x_{1}$ or $x_{2}$, one has the useful results

$$
\begin{aligned}
& \int d x_{2}\left\langle\left\langle X\left(x_{1} ; \tau_{1}\right)\right\rangle\right\rangle=\left\langle X\left(x_{1} ; \tau_{1}\right)\right\rangle \\
& \int d x_{1}\left\langle\left\langle X\left(x_{2} ; \tau_{1}\right)\right\rangle\right\rangle=\left\langle X\left(x_{2} ; 0\right)\right\rangle .
\end{aligned}
$$

Note that although $(\mathrm{C} .14,15)$ look innocuous there is the hidden assumption that the Fourier transforms in (C.10, C.13) can be inverted. We shall return to this question in Appendix E.

The discontinuity in (C.12) is most simply evaluated by splitting into partial fractions. It will turn out to be useful to do this in two ways:

$$
\begin{aligned}
\operatorname{Disc}\left[\frac{1}{\left(x_{1}-x_{\mathrm{Bj}}\right)\left(x_{2}-x_{\mathrm{Bj}}\right)}\right]= & \operatorname{Disc}\left\{\frac{1}{x_{2}-x_{1}}\left[\frac{1}{x_{1}-x_{\mathrm{Bj}}}-\frac{1}{x_{2}-x_{\mathrm{Bj}}}\right]\right\} \\
= & \frac{-i \pi}{x_{2}-x_{1}}\left[\delta\left(x_{1}-x_{\mathrm{Bj}}\right)-\delta\left(x_{2}-x_{\mathrm{Bj}}\right)\right]
\end{aligned}
$$

or

$$
\begin{aligned}
\operatorname{Disc}\left[\frac{1}{\left(x_{1}-x_{\mathrm{Bj}}\right)\left(x_{2}-x_{\mathrm{Bj}}\right)}\right] & =\operatorname{Disc}\left\{\frac{1}{x_{1}+x_{2}-2 x_{\mathrm{Bj}}}\left[\frac{1}{x_{1}-x_{\mathrm{Bj}}}+\frac{1}{x_{2}-x_{\mathrm{Bj}}}\right]\right\}_{(} \\
& =\frac{-i \pi}{x_{1}+x_{2}-2 x_{\mathrm{Bj}}}\left[\delta\left(x_{1}-x_{\mathrm{Bj}}\right)+\delta\left(x_{2}-x_{\mathrm{Bj}}\right)\right] .
\end{aligned}
$$

$$
\gamma^{\alpha} \gamma^{\rho} \gamma^{\beta}-g^{\alpha \rho} \gamma^{\beta}=g^{\rho \beta} \gamma^{\alpha}-g^{\alpha \beta} \gamma^{\rho}-i \varepsilon^{\alpha \rho \beta \sigma} \gamma_{\sigma} \gamma_{5}
$$

is helpful in simplifying the $\gamma$-algebra and will be crucial at a later stage of the analysis.

When using this to simplify $\gamma^{\nu}\left(\not q+x_{2} \not P\right) A\left(\not q+x_{1} \not P\right) \gamma^{\mu}$, and when the piece antisymmetric in $\mu \nu$ is extracted, one can discard all terms of the type $\gamma^{\nu}(\not P$ or $\not q)(P \cdot A$ or $q \cdot A) \gamma^{\mu}$, since they give a vanishing contribution in leading order. To see this, via (10.1.5), such contributions involve terms like

$$
\left(P^{\rho} \text { or } q^{\rho}\right)\left(P_{\alpha} \text { or } q_{\alpha}\right) \varepsilon^{\mu \nu \alpha \sigma}\left\langle P, S_{T}\left|\bar{\psi}(0) \gamma_{\sigma} \gamma_{5} A_{\rho}\left(\tau_{1} n\right) \psi\left(\tau_{2} n\right)\right| P, S_{T}\right\rangle .
$$

Now the matrix element is a pseudotensor. The leading terms linear in $S_{T}$ can then only have the forms $P_{\sigma} S_{\rho}^{T}$ and $S_{\sigma}^{T} P_{\rho}$. But the latter must be absent since in our gauge $A \cdot n=0$. Thus (C.19) involves $P \cdot S_{T}$ or $q \cdot S_{T}$ which both vanish.

Finally, the antisymmetric part of the $\gamma$-matrix expression in (C.12) becomes

$$
g(P \cdot q)\left\{-i \varepsilon^{\mu \nu \rho \sigma} \gamma_{\sigma} \gamma_{5} A_{\rho}(\tau n)\left(x_{1}+x_{2}-2 x_{\mathrm{Bj}}\right)+\left(x_{1}+x_{2}\right) \not h\left[P^{\nu} A^{\mu}(\tau n)-P^{\mu} A^{\nu}(\tau n)\right]\right\} .
$$

Combining this judiciously with (C.16 and 17) we get for the antisymmetric part of (C.12)

$$
\begin{aligned}
W_{G(A)}^{\mu \nu} & =\frac{e_{f}^{2}}{8 P \cdot q} \int d x_{1} d x_{2}\left\{g \delta ( x _ { 1 } - x _ { \mathrm { Bj } } ) \left\langle\left\langle-\varepsilon^{\mu \nu \rho \sigma} \gamma_{\sigma} \gamma_{5} A_{\rho}(\tau n)\right.\right.\right. \\
& \left.\left.-i \not h\left[P^{\nu} A^{\mu}(\tau n)-P^{\mu} A^{\nu}(\tau n)\right]\right\rangle\right\rangle-g \delta\left(x_{2}-x_{\mathrm{Bj}}\right)\left\langle\left\langle\varepsilon^{\mu \nu \rho \sigma} \gamma_{\sigma} \gamma_{5} A_{\rho}(\tau n)\right.\right. \\
& \left.\left.\left.-i \not h\left[P^{\nu} A^{\mu}(\tau n)-P^{\mu} A^{\nu}(\tau n)\right]\right\rangle\right\rangle\right\} .
\end{aligned}
$$

The trivial dependence on $x_{1}, x_{2}$ allows us to use (C.14 and 15) to obtain

$$
\begin{aligned}
W_{G(A)}^{\mu \nu} & =\frac{e_{f}^{2}}{8 P \cdot q} \int d x \delta\left(x-x_{\mathrm{Bj}}\right)\left\{\left\langle-\varepsilon^{\mu \nu \rho \sigma} \gamma_{\sigma} \gamma_{5} A_{\rho}(0)\right.\right. \\
& \left.-i \not h\left[P^{\nu} A^{\mu}(0)-P^{\mu} A^{\nu}(0)\right]\right\rangle-\left\langle\varepsilon^{\mu \nu \rho \sigma} \gamma_{\sigma} \gamma_{5} A_{\rho}(\tau n)\right. \\
& \left.\left.-i \not h\left[P^{\nu} A^{\mu}(\tau n)-P^{\mu} A^{\nu}(\tau n)\right]\right\rangle\right\}
\end{aligned}
$$


Now note that the structures of $\Delta W_{(A)}^{\mu \nu}$ in (C.9) and $W_{G(A)}^{\mu \nu}$ in (C.22) are identical, so we may add them to obtain

$$
\begin{aligned}
\Delta W_{(A)}^{\mu \nu}+W_{G(A)}^{\mu \nu} & =\frac{e_{f}^{2}}{8 P \cdot q} \int d x \delta\left(x-x_{\mathrm{Bj}}\right)\left\{\left\langle\varepsilon^{\mu \nu \rho \sigma} \gamma_{\sigma} \gamma_{5} \vec{D}_{\rho}(\tau n)\right.\right. \\
& -m_{q} \sigma^{\mu \nu}-i g \not h\left[P^{\mu} A^{\nu}(\tau n)-P^{\nu} A^{\mu}(\tau n)\right]+\varepsilon^{\mu \nu \rho \sigma} \gamma_{\sigma} \gamma_{5} \overleftarrow{D}_{\rho}(0) \\
& \left.\left.-m_{q} \sigma^{\mu \nu}+i g \not h\left[P^{\mu} A^{\nu}(0)-P^{\nu} A^{\mu}(0)\right]\right\rangle\right\}
\end{aligned}
$$

where we have introduced

$$
\begin{aligned}
& \vec{D}_{\rho}(z)=i \vec{\partial}_{\rho}-g A_{\rho}(z) \\
& \overleftarrow{D}_{\rho}(z)=-i \overleftarrow{\partial}_{\rho}-g A_{\rho}(z)
\end{aligned}
$$

Consider first the terms involving $\not h$. We can simplify them by analysing the structure of the matrix element

$$
\left\langle P, S_{T}\left|\bar{\psi} \gamma^{\mu} A^{\nu} \psi\right| P, S_{T}\right\rangle
$$

It is a true tensor which can only be built from the vectors $P^{\alpha}, n^{\alpha}$ and

$$
\begin{aligned}
v^{\alpha} & =\varepsilon^{\alpha \rho \sigma \lambda} S_{\rho}^{T} P_{\sigma} n_{\lambda} \quad v^{2}=-1 \\
P \cdot v & =n \cdot v=S^{T} \cdot v=0
\end{aligned}
$$

and the pseudovector $S_{\alpha}^{T}$, and which must be linear in $S_{\alpha}^{T}$. The only possible forms are $P^{\mu} v^{\nu}$ or $P^{\nu} v^{\mu}$ of which the latter cannot occur because of the gauge condition $A \cdot n=0$. It follows that

$$
\left\langle\not P A^{\nu}\right\rangle=\left\langle \$_{T}^{\prime} A^{\nu}\right\rangle=\left\langle\psi A^{\nu}\right\rangle=0
$$

If therefore we write, to leading order,

$$
\gamma^{\mu} A^{\nu}=\left[\not h P^{\mu}+\not P n^{\mu}-\not \$_{T} S_{T}^{\mu}-\not \psi v^{\mu}\right] A^{\nu}
$$

then inside (C.23) only the term $\not P^{\mu}$ contributes. Inside the matrix element we thus have the equivalence

$$
\not P^{\mu} A^{\nu} \Longleftrightarrow \gamma^{\mu} A^{\nu}
$$

We may thus, in (C.23), make the replacement

$$
\not h g\left[P^{\mu} A^{\nu}-P^{\nu} A^{\mu}\right] \rightarrow \gamma^{\mu} g A^{\nu}-\gamma^{\nu} g A^{\mu} .
$$

We may also, as a consequence of (C.8) add $0=\left\langle\vec{\partial}_{\rho}+\overleftarrow{\partial}_{\rho}\right\rangle$ so that e.g.

$$
\left\langle i \gamma^{\mu} g A^{\nu}(0)-i \gamma^{\mu} g A^{\nu}(\tau n)\right\rangle=\left\langle-i \gamma^{\mu} \overleftarrow{D}^{\nu}(0)+i \gamma^{\mu} \vec{D}^{\nu}(\tau n)\right\rangle
$$

Finally, (C.23) becomes:

$$
\begin{aligned}
\Delta W_{(A)}^{\mu \nu}+W_{G(A)}^{\mu \nu} & =\frac{e_{f}^{2}}{8 P \cdot q} \int d x \delta\left(x-x_{\mathrm{Bj}}\right)\left\{\left\langle\varepsilon^{\mu \nu \rho \sigma} \gamma_{\sigma} \gamma_{5} \vec{D}_{\rho}(\tau n)-m_{q} \sigma^{\mu \nu}\right.\right. \\
& \left.+i \gamma^{\mu} \vec{D}^{\nu}(\tau n)-i \gamma^{\nu} \vec{D}^{\mu}(\tau n)\right\rangle+\left\langle\varepsilon^{\mu \nu \rho \sigma} \gamma_{\sigma} \gamma_{5} \overleftarrow{D}_{\rho}(0)\right. \\
& \left.\left.-m_{q} \sigma^{\mu \nu}-i \gamma^{\mu} \stackrel{\leftarrow}{D}^{\nu}(0)+i \gamma^{\nu} \overleftarrow{D}^{\mu}(0)\right\rangle\right\}
\end{aligned}
$$


We shall now demonstrate that both matrix elements in (C.31) vanish as a consequence of the equations of motion

$$
\left[\overrightarrow{\not D}(z)-m_{q}\right] \psi(z)=0 \quad \bar{\psi}(z)\left[\overleftarrow{\not D}(z)-m_{q}\right]=0
$$

The starting point is the $\gamma$-matrix identity which follows from (C.18):

$$
0=-\sigma^{\mu \nu} \gamma^{\rho}+i g^{\nu \rho} \gamma^{\mu}-i g^{\mu \rho} \gamma^{\nu}+\varepsilon^{\mu \nu \rho \sigma} \gamma_{\sigma} \gamma_{5}
$$

Taking the scalar product with $\vec{D}_{\rho}(z)$, acting on $\psi(z)$, and using (C.32) yields

$$
0=\left[-m_{q} \sigma^{\mu \nu}+\varepsilon^{\mu \nu \rho \sigma} \gamma_{\sigma} \gamma_{5} \vec{D}_{\rho}(z)+i \gamma^{\mu} \vec{D}^{\nu}(z)-i \gamma^{\nu} \vec{D}^{\mu}(z)\right] \psi(z)
$$

which is exactly the structure that appears in the first matrix element of (C.31). A similar argument shows that the second matrix element also vanishes.

Thus the miracle is achieved. The unwanted $x \not P$ and mass terms have disappeared as a consequence of the equations of motion when the two parton handbag diagram is combined with the three parton gluon diagram. The result is (10.3.4).

\section{Appendix D - Distribution and two-parton correlation functions for transverse spin}

It is of some interest to consider the distribution and correlation functions associated with the matrix elements of the quark and gluon operators appearing in Appendix $\mathrm{C}$ and to derive certain relationships between them which follow from the equations of motion. The structure of these correlation functions will be further studied in Appendix E.

Consider first $\left\langle\sigma^{\mu \nu}\right\rangle$ in the notation of (C.10). Its leading order tensorial structure can only be $\epsilon^{\mu \nu \alpha \beta} P_{\alpha} S_{\beta}^{T}$. Thus we put

$$
\left\langle\sigma^{\mu \nu}\right\rangle=4 h_{T}(x) \epsilon^{\mu \nu \alpha \beta} P_{\alpha} s_{\beta}^{T}
$$

and find that $\square$

$$
4 h_{T}(x)=\int \frac{d \tau}{2 \pi} e^{i \tau x} \widetilde{h}_{T}(\tau)
$$

where

$$
\tilde{h}_{T}(\tau)=\left\langle P, S_{T}\left|\bar{\psi}(0) \not \gamma_{5} \$_{T} \psi(\tau n)\right| P, S_{T}\right\rangle .
$$

It is $h_{T}(x)$ that is the true analogue of $h_{L}(x)$ of $(10.2 .10)$ and which has a simple parton interpretation in terms of probabilities to find quarks with transverse spin in a transversely polarized nucleon. As we have seen, however, in DIS this term appears multiplied by the quark mass [Eq. (10.1.7)] and in fact eventually cancels out. Nonetheless it is a fundamental distribution reflecting a different aspect of the structure of the nucleon, and, as has been emphasized by [COR 92], can be measured directly in Drell-Yan reactions.

Consider next the matrix element $\left\langle\left\langle\gamma^{\mu} \vec{D}^{\nu}(\tau n)\right\rangle\right\rangle$. In leading order one can only have the tensor structures $P^{\mu} v^{\nu}$ or $P^{\nu} v^{\mu}$ but one can show that the latter term is not permitted by time-reversal invariance. So one can put

$$
\left\langle\left\langle\gamma^{\mu} \vec{D}^{\nu}(\tau n)\right\rangle\right\rangle=M B^{V}\left(x_{1}, x_{2}\right) P^{\mu} v^{\nu}
$$

1) $\operatorname{Our} h_{T}(x)=1 / 2 h_{T}^{C O R}(x)$ for reasons explained after Eq. (10.2.11). 
It follows that

$$
B^{V}\left(x_{1}, x_{2}\right)=\int \frac{d \tau_{1}}{2 \pi} \frac{d \tau_{2}}{2 \pi} e^{i \tau_{2} x_{2}} e^{i \tau_{1}\left(x_{1}-x_{2}\right)} \widetilde{B}^{V}\left(\tau_{1}, \tau_{2}\right)
$$

where

$$
\widetilde{B}^{V}\left(\tau_{1}, \tau_{2}\right)=-\frac{1}{M}\left\langle P, S_{T}\right| \bar{\psi}(0) \not h\left(v \cdot \vec{D}\left(\tau_{1} n\right) \psi\left(\tau_{2} n\right)\left|P, S_{T}\right\rangle .\right.
$$

Note that it can be shown [EFR 84] that as a consequence of time reversal invariance

$$
B^{V}\left(x_{1}, x_{2}\right)=-B^{V}\left(x_{2}, x_{1}\right) .
$$

Now using (C.14) one has

$$
\left\langle i \gamma^{\mu} \vec{D}^{\nu}(\tau n)-i \gamma^{\nu} \vec{D}^{\mu}(\tau n)\right\rangle=i M \int d x_{2} B^{V}\left(x_{1}, x_{2}\right) \varepsilon^{\mu \nu \alpha \beta} P_{\alpha} S_{\beta}^{T}
$$

where we have used

$$
\begin{aligned}
P^{\mu} v^{\nu}-P^{\nu} v^{\mu} & =P^{\mu} \varepsilon^{\nu \alpha \beta \gamma} S_{\alpha}^{T} P_{\beta} n_{\gamma}-P^{\nu} \varepsilon^{\mu \alpha \beta \gamma} S_{\alpha}^{T} P_{\beta} n_{\gamma} \\
& =\varepsilon^{\mu \nu \alpha \beta} P_{\alpha} S_{\beta}^{T} .
\end{aligned}
$$

Finally, consider $\left\langle\left\langle\gamma^{\sigma} \gamma_{5} \vec{D}^{\rho}(\tau n)\right\rangle\right\rangle$. In leading order, one can only have

$$
\left\langle\left\langle\gamma^{\sigma} \gamma_{5} \vec{D}^{\rho}\left(\tau_{1} n\right)\right\rangle\right\rangle=M B^{A}\left(x_{1}, x_{2}\right) P^{\sigma} S_{T}^{\rho}+M B_{T}^{A}\left(x_{1}, x_{2}\right) P^{\rho} S_{T}^{\sigma}
$$

with

$$
B^{A}\left(x_{1}, x_{2}\right)=\int \frac{d \tau_{1}}{2 \pi} \frac{d \tau_{2}}{2 \pi} e^{i \tau_{2} x_{2}} e^{i \tau_{1}\left(x_{1}-x_{2}\right)} \widetilde{B}^{A}\left(\tau_{1}, \tau_{2}\right)
$$

where

$$
\widetilde{B}^{A}\left(\tau_{1}, \tau_{2}\right)=-\frac{1}{M}\left\langle P, S_{T}\left|\bar{\psi}(0) \not h \gamma_{5}\left[S^{T} \cdot \vec{D}\left(\tau_{1} n\right)\right] \psi\left(\tau_{2} n\right)\right| P, S_{T}\right\rangle
$$

and

$$
B_{T}^{A}\left(x_{1}, x_{2}\right)=\int \frac{d \tau_{1}}{2 \pi} \frac{d \tau_{2}}{2 \pi} e^{i \tau_{2} x_{2}} e^{i \tau_{1}\left(x_{1}-x_{2}\right)} \widetilde{B}_{T}^{A}\left(\tau_{1}, \tau_{2}\right)
$$

where

$$
\widetilde{B}_{T}^{A}\left(\tau_{1}, \tau_{2}\right)=-\frac{1}{M}\left\langle P, S_{T}\left|\bar{\psi}(0) \$_{T} \gamma_{5} n\left[n \cdot D\left(\tau_{1} n\right)\right] \psi\left(\tau_{2} n\right)\right| P, S_{T}\right\rangle
$$

From time reversal invariance one has [EFR 84]

$$
B^{A}\left(x_{1}, x_{2}\right)=B^{A}\left(x_{2}, x_{1}\right) .
$$

Note that in (D.12), because of the gauge condition,

$$
n \cdot \vec{D}\left(\tau_{1} n\right)=i n^{\mu} \partial_{\mu}
$$

so that the $\tau_{1}$ integration can be done yielding

$$
\begin{aligned}
& B_{T}^{A}\left(x_{1}, x_{2}\right)=-\frac{i}{M} \delta\left(x_{1}-x_{2}\right) \int \frac{d \tau_{2}}{2 \pi} e^{i \tau_{2} x_{2}} \\
& \quad \times\left\langle P, S_{T}\left|\bar{\psi}(0) \$_{T}^{\prime} \gamma_{5} n^{\alpha} \partial_{\alpha} \psi\left(\tau_{2} n\right)\right| P, S_{T}\right\rangle .
\end{aligned}
$$

It is fairly obvious, and is shown in Appendix E, that the derivative $n \cdot \partial$ acting on $\psi$ is equivalent to $\partial / \partial \tau_{2}$ which may then be switched so as to act on the exponential, yielding

$$
B_{T}^{A}\left(x_{1}, x_{2}\right)=4 \delta\left(x_{1}-x_{2}\right) x_{2} f_{T}\left(x_{1}\right)
$$


where $f_{T}$ is the function appearing in (10.3.6).

Using (C.14) and (D.9) we have

$$
\left\langle\varepsilon^{\mu \nu \rho \sigma} \gamma_{\sigma} \gamma_{5} \vec{D}_{\rho}\left(\tau_{1} n\right)\right\rangle=M \int d x_{2}\left\{B_{T}^{A}\left(x_{1}, x_{2}\right)-B^{A}\left(x_{1}, x_{2}\right)\right\} \varepsilon^{\mu \nu \alpha \beta} P_{\alpha} S_{\beta}^{T} .
$$

The vanishing of the matrix element of (C.34) thus implies, using (D.1, 7 and 17)

$$
\int d x_{2}\left\{B_{T}^{A}\left(x_{1}, x_{2}\right)-B^{A}\left(x_{1}, x_{2}\right)+i B^{V}\left(x_{1}, x_{2}\right)\right\}=\frac{4 m}{M} h_{T}\left(x_{1}\right) .
$$

Taking account of (D.17) this becomes:

$$
\int d x_{2}\left\{i B^{V}\left(x_{1}, x_{2}\right)-B^{A}\left(x_{1}, x_{2}\right)\right\}=\frac{4 m}{M} h_{T}\left(x_{1}\right)-4 x_{1} f_{T}\left(x_{1}\right) .
$$

Use of the equations of motion acting to the left upon $\bar{\psi}(0)$ eventually leads to an analogous relationship:

$$
\int d x_{1}\left\{i B^{V}\left(x_{1}, x_{2}\right)+B^{A}\left(x_{1}, x_{2}\right)\right\}=\frac{4 m}{M} h_{T}\left(x_{2}\right)+4 x_{2} f_{T}\left(x_{2}\right) .
$$

\section{Appendix E - The Burkhardt-Cottingham and the Efremov-Leader-Teryaev Sum Rules in the QCD Field Theoretic Model}

We shall show how the above sum rules can be derived from a careful study of the structure of the matrix elements involved in Section 10 and in Appendix D. The other ingredient is the equation of motion for $\psi(z)$. Also one has to make assumptions about the invertability of certain Fourier transforms. One sees very clearly where the weak point is in the 'proof' of these sum rules (see Sections 5.4 and 5.5). In the case of the EfremovLeader-Teryaev sum rule, the original derivation [EFR 84] was a little unsatisfactory, because it appealed to a particular reaction to derive properties inherent to the nucleon. The present derivation deals only with nucleon matrix elements.

Consider first the forward matrix element of the bilocal operator

$$
\bar{\psi}(0) \gamma^{\mu} \gamma_{5} \psi(z)
$$

on the light cone $z^{2}=0$. Its most general form is

$$
\frac{1}{M}\left\langle\bar{\psi}(0) \gamma^{\mu} \gamma_{5} \psi(z)\right\rangle_{P, S}=A_{1} S^{\mu}+(z \cdot S) A_{2} P^{\mu}+(z \cdot S) A_{3} z^{\mu}
$$

where $\langle\ldots\rangle_{P, S}$ is short for $\langle P, S|\ldots| P, S\rangle$. The scalar functions $A_{1,2,3}$ are functions only of $z \cdot P$.

From (E.1) we deduce

$$
\begin{aligned}
\frac{1}{M}\left\langle\bar{\psi}(0) \gamma^{\mu} \gamma_{5} \partial^{\nu} \psi(z)\right\rangle_{P, S} & =A_{1}^{\prime} S^{\mu} P^{\nu}+A_{2} P^{\mu} S^{\nu}+A_{3} z^{\mu} S^{\nu} \\
& +(z \cdot S)\left[A_{2}^{\prime} P^{\mu} P^{\nu}+A_{3}^{\prime} z^{\mu} P^{\nu}+A_{3} g^{\mu \nu}\right]
\end{aligned}
$$

where

$$
A^{\prime} \equiv \frac{d A(z \cdot P)}{d(z \cdot P)}
$$


We now put $z^{\mu}=\tau n^{\mu}$ as required in the matrix elements involved in Section 10 and Appendix D. Then

$$
\frac{1}{M}\left\langle\bar{\psi}(0) \gamma^{\mu} \gamma_{5} \psi(\tau n)\right\rangle_{P, S}=A_{1} S^{\mu}+\tau(n \cdot S)\left[A_{2} P^{\mu}+\tau A_{3} n^{\mu}\right]
$$

where now $A_{i}=A_{i}(\tau)$, and

$$
\begin{aligned}
\frac{1}{M}\left\langle\bar{\psi}(0) \gamma^{\mu} \gamma_{5} \partial^{\nu} \psi(\tau n)\right\rangle_{P, S} & =A_{1}^{\prime} S^{\mu} P^{\nu}+A_{2} P^{\mu} S^{\nu}+\tau\left\{A_{3} n^{\mu} S^{\nu}\right. \\
& \left.+(n \cdot S)\left[A_{2}^{\prime} P^{\mu} P^{\nu}+\tau A_{3}^{\prime} n^{\mu} P^{\nu}+A_{3} g^{\mu \nu}\right]\right\}
\end{aligned}
$$

We assume that all scalar functions are such that $\tau A(\tau) \rightarrow 0$ as $\tau \rightarrow 0$ for all terms occurring in (E.4 and 5). Then at $\tau=0$ we have the simple structures

$$
\frac{1}{M}\left\langle\bar{\psi}(0) \gamma^{\mu} \gamma_{5} \psi(0)\right\rangle_{P, S}=A_{1}(0) S^{\mu}
$$

and

$$
\frac{1}{M}\left\langle\bar{\psi}(0) \gamma^{\mu} \gamma_{5} \partial^{\nu} \psi(0)\right\rangle_{P, S}=A_{1}^{\prime}(0) S^{\mu} P^{\nu}+A_{2}(0) P^{\mu} S^{\nu}
$$

We shall also require, from (E.5)

$$
\frac{1}{M}\left\langle\bar{\psi}(0) \gamma_{5} \not \partial \psi(\tau n)\right\rangle_{P, S}=-\tau(n \cdot S)\left[M^{2} A_{2}^{\prime}+5 A_{3}+\tau A_{3}^{\prime}\right]
$$

so that at $\tau=0$

$$
\frac{1}{M}\left\langle\bar{\psi}(0) \gamma_{5} \not \partial \psi(0)\right\rangle_{P, S}=0 .
$$

Finally note, from (E.5) that

$$
\begin{aligned}
\frac{1}{M}\left\langle\bar{\psi}(0) \gamma^{\mu} \gamma_{5} n \cdot \partial \psi(\tau n)\right\rangle_{P, S} & =A_{1}^{\prime} S^{\mu}+(n \cdot S)\left[\left(A_{2}+\tau A_{2}^{\prime}\right) P^{\mu}+\tau\left(2 A_{3}+\tau A_{3}^{\prime}\right) n^{\mu}\right. \\
& =\frac{1}{M} \frac{d}{d \tau}\left\langle\bar{\psi}(0) \gamma^{\mu} \gamma_{5} \psi(\tau n)\right\rangle_{P, S}
\end{aligned}
$$

a result used in Appendix D.

Consider now the gluonic matrix element

$$
1 / M\left\langle\bar{\psi}(0) \gamma^{\mu} \gamma_{5} g A^{\nu}(z) \psi(z)\right\rangle_{P, S}
$$

with $z=\tau n$. Its most general form is

$$
\begin{aligned}
\tau(S \cdot n)[ & \left.B_{1} P^{\mu} P^{\nu}+\tau B_{2} P^{\mu} n^{\nu}+\tau B_{3} n^{\mu} P^{\nu}+\tau^{2} B_{4} n^{\mu} n^{\nu}\right] \\
& +B_{5} S^{\mu} P^{\nu}+B^{6} P^{\mu} S^{\nu}+\tau B_{7} S^{\mu} n^{\nu}+\tau B_{8} n^{\mu} S^{\nu}
\end{aligned}
$$

The gauge condition $n_{\mu} A^{\mu}=0$ implies that

$$
B_{5}=0, \quad \tau B_{1}=-B_{6}, \quad \tau B_{3}=-B_{8}
$$

so that

$$
\begin{aligned}
\frac{1}{M}\left\langle\bar{\psi}(0) \gamma^{\mu} \gamma_{5} g A^{\nu}(\tau n) \psi(\tau n)\right\rangle_{P, S} & =\tau B_{1}\left[(S \cdot n) P^{\mu} P^{\nu}-P^{\mu} S^{\nu}\right] \\
+\tau(S \cdot n)\left[B_{2} P^{\mu} n^{\nu}\right. & \left.+\tau B_{4} n^{\mu} n^{\nu}\right] \\
+ & \tau^{2} B_{3}\left[(S \cdot n) n^{\mu} P^{\nu}-n^{\mu} S^{\nu}\right]+\tau B_{7} S^{\mu} n^{\nu}
\end{aligned}
$$


Notice the crucial feature, that the imposition of the gauge condition, together with the assumptions about the vanishing of products like $\tau B(\tau)$ as $\tau \rightarrow 0$, leads to the vanishing of (E.13) at $\tau=0$ i.e.

$$
\left\langle\bar{\psi}(0) \gamma^{\mu} \gamma_{5} g A^{\nu}(0) \psi(0)\right\rangle_{P, S}=0 .
$$

This result will be crucial for deriving the Efremov-Leader-Teryaev sum rule.

Let us now relate some of the above coefficients to the functions occurring in the discussion of $g_{1}$ and $g_{2}$.

From (10.2.11) and (E.4) we have

$$
\widetilde{h}_{L}(\tau)=A_{1}(\tau)+\tau A_{2}(\tau) .
$$

From (10.3.7) and (E.4)

$$
\tilde{f}_{T}(\tau)=A_{1}(\tau) .
$$

Then from (10.2.13) and (10.2.10), if the Fourier transforms can be inverted,

$$
\begin{aligned}
\int_{0}^{1} d x g_{1}(x) & =\frac{e_{f}^{2}}{8} \widetilde{h}_{L}(0) \\
& =\frac{e_{f}^{2}}{8} A_{1}(0) \quad \text { by }(\mathrm{E} .15) .
\end{aligned}
$$

Similarly, from (10.3.8 and 6)

$$
\begin{aligned}
\int_{0}^{1} d x\left[g_{1}(x)+g_{2}(x)\right] & =\frac{e_{f}^{2}}{8} \widetilde{f}_{T}(0) \\
& =\frac{e_{f}^{2}}{8} A_{1}(0) \quad \text { by (E.16). }
\end{aligned}
$$

Equations (E.17 and 18) imply the Burkhardt-Cottingham sum rule

$$
\int_{0}^{1} d x g_{2}(x)=0
$$

We have already discussed in Section 5.4 why the above derivation may fail because of the non-invertability of the Fourier transforms. We turn now to the Efremov-LeaderTeryaev sum rule.

Consider first Eq. (D.20) which followed from the equations of motion. Integrating over $x_{1}$, using (D.6), (D.10) and (10.3.6) there results:

$$
\widetilde{B}^{A}(0,0)=\left.i \frac{d \widetilde{f}_{T}}{d \tau}\right|_{\tau=0}
$$

where we have taken the quark mass to be zero for simplicity and where we have taken, on the basis of (10.3.6),

$$
4 x f_{T}(x)=i \int \frac{d \tau}{2 \pi} e^{i \tau x} \frac{d \tilde{f}_{T}}{d \tau}(\tau) .
$$

Now because of (E.14), from (D.11)

$$
\widetilde{B}^{A}(0,0)=-\frac{i}{M}\left\langle\bar{\psi}(0) \not h \gamma_{5}\left(S_{T} \cdot \partial\right) \psi(0)\right\rangle_{P, S}
$$

so that via (E.7)

$$
\widetilde{B}^{A}(0,0)=i A_{2}(0) .
$$


Use of this and (E.16) in (E.20) yields

$$
A_{2}(0)=\left.\frac{d}{d \tau} A_{1}(\tau)\right|_{\tau=0}=A_{1}^{\prime}(0) .
$$

Now by arguments similar to those that lead to (E.21), we have

$$
\begin{array}{rlrl}
\frac{8}{e_{f}^{2}} \int_{0}^{1} d x x g_{1}(x) & =\left.i \frac{\widetilde{h}_{L}(\tau)}{d \tau}\right|_{\tau=0} & \\
& =i\left[A_{1}^{\prime}(0)+A_{2}(0)\right] & & \text { by }(\mathrm{E} .15) \\
& =2 i A_{1}^{\prime}(0) & & \text { by }(\mathrm{E} .23) .
\end{array}
$$

Similarly we have

$$
\begin{aligned}
\frac{8}{e_{f}^{2}} 2 \int_{0}^{1} d x x\left[g_{1}(x)\right. & \left.+g_{2}(x)\right]=\left.2 i \frac{d}{d \tau} \tilde{f}_{T}(\tau)\right|_{\tau=0} \\
& =2 i A_{1}^{\prime}(0) \quad \text { by }(\mathrm{E} .16) .
\end{aligned}
$$

Subtracting (E.24) from (E.25) yields the Efremov-Leader-Teryaev sum rule

$$
\int_{0}^{1} d x x\left[g_{1}(x)+2 g_{2}(x)\right]=0 .
$$

The same caveats apply to this 'proof' as do to the Burkhardt-Cottingham sum rule.

\section{Acknowledgements}

The authors are grateful for the hospitality and support of the Theory Division, CERN, the Physics Department, Birkbeck College and the Dipartimento di Fisica Teorica, University of Torino. They wish to acknowledge helpful discussions with A. Anselm, D. Diakonov, S. Forte, P. Hoodbhoy, V. Hughes, B. Ioffe, S. Kulagin, G. Mallot, P. Mulders, P. Nason, R. Sassot, T. Sloan, G. Smirnov, K. Sridhar, O. Teryaev and M. Velasco.

M.A. is grateful to the British Council for financial support. A.V.E. thanks the Royal Society of London, the U.K. SERC, the International Science Foundation (Grant RFE 000) and the Russian Foundation for Fundamental Investigation (Grant 93-02-3811), for financial support. E.L. is grateful to the U.K. SERC and the INFN for support. He also acknowledges the warm hospitality of the European Centre for Theoretical Studies, Trento.

\section{References}

[ABJ 69] S.I. Adler, Phys. Rev. 177 (1969) 2426; J.S. Bell and R. Jackiw, N.C. 51A (1969) 47.

[ADA 94] D. Adams et al., Phys. Lett. B329 (1994) 399.

[ADA 94a] D. Adams et al., Phys. Lett. B336 (1994) 125.

[ADE 93] B. Adeva et al., Phys. Lett. B302 (1993) 533.

[ADE 94] B. Adeva et al., Phys. Lett. B320 (1994) 400. 
[AHM 76] M.A. Ahmed and G.G. Ross, Nucl. Phys. B111 (1976) 441.

[AHR 87] L.H. Ahrens et al., Phys. Rev. D35 (1987) 785.

[ALG 78] M.J. Alguard et al., Phys. Rev. Lett. 41 (1978) 70.

[ALT 77] G. Altarelli and G. Parisi, Nucl. Phys. B126 (1977) 298.

[ALT 82] G. Altarelli, Phys. Rep. 81 (1982) 1.

[ALT 88] G. Altarelli and G.G. Ross, Phys. Lett. B212 (1988) 391.

[ALT 94] G. Altarelli, P. Nason and G. Ridolfi, Phys. Lett. B320 (1994) 152; Erratum, B325 (1994) 538.

[ALT 94a] R. Altmeyer et al., Phys. Rev. D49 (1994) 3087.

[AMA 92] NMC Collaboration: P. Amaudruz et al., Phys. Lett. B295 (1992) 159.

[ANS 79] M. Anselmino, Phys. Rev. D19 (1979) 2803.

[ANS 89] M. Anselmino, B.L. Ioffe and E. Leader, Yad. Fiz 49 (1989) 214 [Sov. J. Nucl. Phys. 49 (1989) 136].

[ANS 89a] A.A. Anselm and A. Johansen, JETP Lett. 49 (1989) 214.

[ANS 92] M. Anselmino and E. Leader, Phys. Lett. B293 (1992) 216.

[ANS 92a] A.A. Anselm, Phys. Lett. B291 (1992) 455.

[ANS 93] M. Anselmino, P. Gambino and J. Kalinowski, Z. Phys. C64 (1994) 267.

[ANT 93] D.L. Anthony et al., Phys. Rev. Lett. 71 (1993) 759.

[ASH 88] J. Ashman et al., Phys. Lett. B206 (1988) 364.

[ASH 89] J. Ashman et al., Nucl. Phys. B328 (1989) 1.

[BAG 90] B. Bagchi, A. Lahiri, J. Phys. G16 (1990) L239.

[BAI 82] See e.g. Chapter 4 of D. Bailin, 'Weak Interactions', Sussex University Press (1982). Note that our sign convention for F and D is opposite to Bailin's.

[BAL 90] I.I. Balitsky, V.M. Braun and A.V. Kolesnichenko, Phys. Lett. B242 (1990) 245.

[BAL 93] I.I. Balitsky, V.M. Braun and A.V. Kolesnichenho, Penn State University Preprint, hep-ph 9310316.

[BAR 79] J.A. Bartelski, Acta Phys. Pol. B10 (1979) 10; 923.

[BAS 91] S.D. Bass, B.L. Ioffe, N.N. Nikolaev and A.W. Thomas, J. Moscow Phys. Soc. 1 (1991) 317.

[BAS 93] S.D. Bass, A.W. Thomas, J. Phys. C19 (1993) 925.

[BAU 83, 88] G. Baum et al., Phys. Rev. Lett. 51 (1983) 1135; V.W. Hughes et al., Phys. Lett. 212 (1988) 511.

[BER 89] E. Berger and J. Qiu, Phys. Rev. D40 (1989) 778 and 3128; S. Gupta, D. Indumathi and M.V.N. Murthy, Z. Phys. C42 (1989) 493; H.Y Cheng and S.N. Lai, Phys. Rev. D41 (1990) 91; C. Bourrely, J. Ph. Guillet and J. Soffer, Nucl. Phys. B361 (1991) 72; R.M. Godbole, S. Gupta and K. Sridhar, Phys. Lett. B225 (1991) 120; A.P. Contogouris et al., Phys. Lett. B304 (1993) 329.

[BIL 75] S.M. Bilenky, N.A. Dadajan and E.H. Hristowa, Sov. J. Nucl. Phys. 21 (1975) 657.

[BJO 65] J.D. Bjorken and S.C. Drell, 'Relativistic Quantum Fields', New York, Mc Graw-Hill, 1965.

[BJO 66] J.D. Bjorken, Phys. Rev. 148 (1966) 1467.

[BOU 80] C. Bourrely, E. Leader and J. Soffer, Phys. Rep. 59 (1980) 95.

[BRO 88] S. Brodsky, J. Ellis and M. Karliner, Phys. Lett. B206 (1988) 309; J. Ellis and M. Karliner, Phys. Lett. B213 (1988) 73.

[BUR 70] H. Burkhardt and W.N. Cottingham, Ann. Phys. 56 (1970) 453.

[BUR 92] V.D. Burkert and B.L. Ioffe, Phys. Lett. B296 (1992) 223. 
[BUR 93] V.D. Burkert and B.L. Ioffe, CEBAF preprint, CEBAF-PR-93-034, October 1993.

[CAH 78] R.N. Cahn and F.G. Gilman, Phys. Rev. D17 (1978) 1313.

[CAL 76] C. Callan, R. Dashen, D. Gross, Phys. Lett 63B (1976) 334.

[CAR 72] C.E. Carlson and Wu-ki Tung, Phys. Rev. D5 (1972) 721; J.S. Bell and R. Jackiw, N.C. 51 A (1969) 47.

[CAR 88] R.D. Carlitz, J.C. Collins and A.H. Mueller, Phys. Lett. B214 (1988) 229.

[CAR 92] R.D. Carlitz and R.S. Willey, Phys. Rev. D45 (1992) 2323.

[CHE 89] T.P. Cheng and L.F. Li, Phys. Rev. Lett. 62 (1989) 1441.

[CIO 93] C. Ciofi degli Atti, S. Scarpetta, E. Pace and G. Salmè, Phys. Rev. C48 (1993) R968.

[CLO 88] F.E. Close and R.G. Roberts, Phys. Rev. Lett. 40 (1988) 1471.

[CLO 89] F. Close and L. Milner, ORNL-report 31770 (1989); L. Frankfurt et al., Phys. Lett. B230 (1989) 141; A.V. Efremov and V. Alizade, Prepr. JINR E-2-91-465 (1991); M. Veltri et al., Proceedings of 'Physics at HERA' (Hamburg, 1991) Vol. 1, p. 447.

[CLO 93] F.E. Close and R.G. Roberts, Phys. Lett. B316 (1993) 165. See also plenary talk: 13th Intl. Conf. on Particles and Nuclei, PANIC 93, July 1993, Perugia, Italy.

[CON 91] A.P. Contogouris and S. Papadopoulos, Phys. Lett B260 (1991) 204; M. Glück and W. Vogelsang, Phys. Lett B277 (1992) 515; P. Matheus and R. Ramachandran, Z. Phys. C53 (1992) 305.

[COR 88] J.L. Cortes and B. Pire, Phys. Rev. D38 (1988) 3586; G. Ballochi et al., HELP, CERN/LEPC 89-90 and LEPC/M88 (1989); M.A. Doncheshi and R.W. Robinett, Phys. Lett. B248 (1990) 188; A.P Contogouris, S. Papadopoulos and B. Kamal, Phys. Lett. B246 (1990) 523; K. Culter et al., HERMES, DESY/PRE 90-1 (1990)

[COR 92] J.L. Cortes, B. Pire and J.P. Ralston, Z. Phys. C55 (1992) 409.

[CRA 83] N.S. Craigie, K. Hidaka, M. Jacob and F.M. Renard, Phys. Rep. 99 (1983) 69.

[CRO 83] C. Cronström and J. Mickelsson, J. Math. Phys. 24 (1983) 2528. See also [JAC 85].

[DAL 56] R.H. Dalitz, Int. School of Physics "Enrico Fermi", Corse, Vol. 33, Academic Press, 1956.

[DEF 93] D.E. de Florian et al., La Plata preprint 93-03 (1993).

[DER 73] E. Derman, Phys. Rev. D7 (1973) 2755.

[DOR 91] A.E. Dorokhov and N.I. Kochelev, Phys. Lett. B259 (1991) 335.

[DOR 93] A.E Dorokhov, N.I Kochelev and A.Yu. Zubov, Int. J. of Mod. Phys. A8 (1993) 603.

[DRE 64] S.D. Drell and J.D. Walecka, Ann. Phys. 28 (1964) 18.

[DRE 66] S.D. Drell and A.C. Hearn, Phys. Rev. Lett. 16 (1966) 908.

[DUM 83] O. Dumbrais et al., Nucl. Phys. B216 (1983) 277.

[DYA 81] D.I. Dyakonov and M.I. Eides, Sov. Phys. JETP 54 (1981) 2.

[EFR 78] A.V. Efremov, Sov. J. Nucl. Phys. 28 (1978) 83; R.H. Dalitz, G.R. Goldstein and R. Marshall, Z. Phys. C42 (1989) 441; A.V. Efremov, L. Mankiewicz and N.A. Törnqvist, Phys. Lett. B284 (1992) 394.

[EFR 84] A.V. Efremov and O.V. Teryaev, Sov. J. Nucl. Phys. 39 (1984) 962 [Yad. Fiz. 39 (1984) 1517].

[EFR 87] A.V.Efremov and O.V. Teryaev, Phys. Lett. B200 (1987) 363. 
[EFR 88] A.V. Efremov and O.V. Teryaev, JINR, Report No. E2-88-287 (1988); unpublished.

[EFR 90] A.V. Efremov, J. Soffer and N. Törnqvist, Phys. Rev. Lett. 64 (1990) 1495; A.V. Efremov, J. Soffer and O.V. Teryaev, Nucl. Phys. B396 (1990) 97.

[EFR 91] A.V. Efremov, J. Soffer and N. Törnqvist, Phys. Rev. D44 (1991) 1369.

[EFR 91a] A.V. Efremov and V. Karotkijan, Sov. J. Nucl. Phys. 54 (1991) 937.

[EFR 92] A.V. Efremov, 'Polarization Dynamics in Nuclear and Particle Physics' (Proceedings of the 2nd Adriatico Research Conference, Trieste, 1992), Ed. A.O. Barut et al., World Scientific, Singapore, 1993, p. 218.

[EFR 92a] A.V. Efremov, L. Mankiewicz and N.A. Törnqvist, Phys. Lett. B284 (1992) 394.

[EFR 94] A.V. Efremov, 'A model for the estimation of jet handedness', (to be published).

[EFR 94a] A.V. Efremov and E. Leader, BNL preprint BNL-60734 (1994); submitted to Phys. Rev. Lett.

[ELL 74] J. Ellis and R.L. Jaffe, Phys. Rev. D9 (1974) 1444; Erratum, D10 (1974) 1669.

[ELL 88] J. Ellis and M. Karliner, Phys. Lett. B213 (1988) 73.

[ELL 93] J. Ellis and M. Karliner, Phys. Lett. B313 (1993) 131; See also Plenary talk at PANIC-93.

[EPE 92] L.N. Epele, H. Fanchiotti, C.A. Garcia Canal and R. Sassot, Phys. Lett. 287 (1992) 247.

[EPE 93] L.N. Epele, H. Fanchiotti, C.A. Garcia Canal, E. Leader and R. Sassot, Birkbeck College Preprint, October 1993.

[FAD 76] L.D. Faddeev, 'Non-local field theories', Proceedings of Alushta Conference on Nonlocal and Nonlinear Theories, Dubna, 1976.

[FEY 72] R.P. Feynman, 'Photon-hadron Interactions', Benjamin, Reading, MA, 1972.

[FOR 90] S. Forte and E. Shuryak, Nucl. Phys. B357 (1990) 153; S. Forte, Phys. Lett. B224 (1989) 189 and Nucl. Phys. B331 (1990) 1.

[GER 66] S. Gerasimov, Sov. J. Nucl. Phys. 2 (1966) 930.

[GLU 88] M. Glück and E. Reya, Z. Phys. C39 (1988) 569; J.P. Guillet, Z. Phys. C39 (1988) 75; G. Altarelli and W.J. Stirling, Particle World 1 (1989) 40; M. Glück, E. Reya and W. Vogelsang, Nucl. Phys. B351 (1991) 579.

[HEI 73] R.L. Heimann, Nucl. Phys. B64 (1973) 429.

[HEL 91] See e.g. K. Heller, 'High Energy Spin Physics' (Proc. of 9th Int. Symp., Bonn, 1990), Ed K.-N. Althoff and W. Meyer, Springer, 1991, Vol .1, p.97; S. Nurushev, 'Frontier of High Energy Spin Physics' (Proc. of 10 Int. Symp. on High Energy Spin Physics, Nagoya, Japan, 1992), Ed. Hasegawa T. et al., Yamada Sci. Foundation and Univ. Acad. Press. Inc., 1993, Tokyo, Japan, p. 311

[HEY 72] A.J.G. Hey and J.E. Mandula, Phys. Rev. D5 (1972) 2610.

[HSU 88] S.Y. Hsuch et al., Phys. Rev. D38 (1988) 2056.

[IOF 84] B.L. Ioffe, V .A. Khoze and L.N. Lipatov, 'Hard processes', North-Holland, Amsterdam, 1984.

[IOF 92] B.L. Ioffe and A.Yu. Khodjamirian, Preprint ITEP-41 (1992).

[JAC 76] R. Jackiw, G. Rebbi, Phys. Rev. Lett. 37 (1976) 172.

[JAC 85] R. Jackiw, 'Current algebra and anomalies', World Scientific, Singapore (1985).

[JAC 89] J.D. Jackson, G.G. Ross and R.G. Roberts, Phys. Lett. B226 (1989) 159. 
[JAF 90] R.L. Jaffe, Comments Nucl. Phys. 19 (1990) 239.

[JAF 91] R.L. Jaffe and Xiangdong Ji, Phys. Rev. D43 (1991) 724.

[JEN 91] E. Jenkins, Nucl. Phys. B354 (1991) 24.

[JOS 77] A.S. Joshipura and P. Roy, Ann. Phys. 104 (1977) 440.

[KAL 89] P. Kalyniak, M.K. Sundaresan and P.I.S. Watson, Phys. Lett. B216 (1989) 397.

[KAU 77] J. Kaur, Nucl. Phys. B128 (1977) 219.

[KOD 79] J. Kodaira, S. Matsuda, K. Sasaki and T. Uematsu, Nucl. Phys. B159 (1979) 99; J. Kodaira, Nucl. Phys. B165 (1979) 129; J. Kodaira, S. Matsuda, T. Muta, T. Uematsu and K. Sasaki, Phys. Rev. D20 (1979) 627. Note that our covariant spin vector $S_{\mu}$ is $1 /(2 M)$ times the spin vector in the above papers.

[KOD 80] J. Kodaira, Nucl. Phys. B165 (1980) 129.

[KOG 75] J. Kogut and L. Suskind, Phys. Rev. D11 (1975) 3583.

[KOT 70] A. Kotanski, Acta Physica Polonica B1 (1970) 45.

[KUH 90] J.H. Kühn and V.I. Zakharov, Phys. Lett. B252 (1990) 615.

[LAM 82] C.S.Lam and B. Li, Phys. Rev. D28 (1982) 683.

[LAM 89] B. Lampe, Phys. Lett. B227 (1989) 469.

[LAR 91] S.A. Larin and J.A.M. Vermaseren, Phys. Lett. B259 (1991) 345.

[LAR 94] S.A. Larin, Phys. Lett. B334 (1994) 192.

[LEA 85] See, e.g., E. Leader and E. Predazzi, 'An Introduction to Gauge Theories and the New Physics', Cambridge University Press (1985).

[LEA 88] E. Leader and M. Anselmino, Z. Phys. C41 (1988) 239.

[LEA 88a] E. Leader and M. Anselmino, Santa Barbara Preprint NSF-88-142, July 1988, unpublished.

[LEA 88b] E. Leader and P. Ratcliffe, unpublished.

[LEA 93] E. Leader and K. Sridhar, Phys. Lett. B311 (1993) 324.

[LEA 94] E. Leader and E. Predazzi, 'An Introduction to Gauge Theories and Modern Particle Physics', Cambridge University Press, in press.

[LEA 94a] E. Leader and K. Sridhar, Nucl. Phys. B419 (1994) 3

[LIP 90] H. Lipkin, Phys. Lett. B237 (1990) 130.

[MAN 90] J.E. Mandula, Phys. Rev. Lett. 65 (1990) 1403.

[MAN 90a] L. Mankiewicz and A. Schafer, Phys. Lett. B242 (1990) 455.

[MAN 91] A.V. Manohar, Phys. Lett. B255 (1991) 579.

[MAN 91a] L. Mankiewicz, Phys. Rev. D43 (1991) 733.

[MAN 92] J.E. Mandula, 'Polarization Dynamics in Nuclear and Particle Physics' (Proceedings of the 2nd Adriatico Research Conference, Trieste, 1992), Ed. A.O. Barut et al., World Scientific, Singapore, 1993, p. 227.

[MAT 92] P. Mathews and V. Ravindran, Int. J. Mod. Phys. A7 (1992) 309.

[MAT 92b] P. Mathews and V. Ravindran, Phys. Lett. B278 (1992) 175.

[NAC 77] O. Nachtmann, Nucl. Phys. B127 (1977) 314.

[NAS 71] C. Nash, Nucl. Phys. B31 (1971) 419.

[NAS 93] We are very grateful to P. Nason for supplying values of $\alpha_{s}\left(Q^{2}\right)$ which are based upon next-to-leading order $Q^{2}$-dependence with normalisation to $\alpha_{s}\left(M_{Z}\right)=$ $0.116 \pm 0.01$ and exact matching at heavy quark thresholds. The error at $Q^{2}=M_{Z}^{2}$ reflects the disagreement between LEP and DIS values. See S. Catani, Proc. EPS Conf, Marseille, July 1993.

[NIK 73] N.N. Nikolaev, M.A. Shifman and M.Z. Shmatikov, JETP 18 (1973) 39.

[PDG 92] Particle Data Group, Phys. Rev. D45 (1992). 
[PRE 88] G. Preparata and J. Soffer, Phys. Rev. Lett. 61 (1988) 1167

[QIU 91] J. Qiu and G. Sterman, Phys. Rev. Lett. 67 (1991) 2264; Nucl. Phys. B378 (1992) 52.

[RAL 79] J. Ralston and D.E. Soper, Nucl. Phys. B152 (1979) 109. The reader is warned that there is a term $\gamma_{5}\left[\not k_{A}, \mathcal{A}\right]$ where $\mathcal{A}^{\mu}=\left(k_{A} \cdot S_{A}\right) P_{A}^{\mu}-\left(k_{A} \cdot P_{A}\right) S_{A}^{\mu}$, missing from what was meant to be the most general expression for the tensor structure of $\Phi$. This term is not important for the analysis in [RAL 79]. (E. Leader, private communication to the authors, unpublished.)

[RAM 88] G.P Ramsey, D. Richards and D. Sivers, Phys. Rev. D37 (1988) 3140; Z. Kunst, Phys. Lett B218 (1989) 243; G.P. Ramsey and D. Sivers, Phys. Rev. D43 (1991) 2861; M.A. Doncheshi, R.W. Robinett and L. Weinhauf, Phys. Rev. D44 (1991) 2717; P. Chiappetta and G. Nardulli, Z. Phys. C51 (1991) 435.

[RAT 86] P.G. Ratcliffe, Nucl. Phys. B264 (1986) 493.

[RAT 87] P. Ratcliffe, Phys. Lett. B192 (1987) 180.

[RAV 92] V. Ravishankar, Nucl. Phys. B374 (1992) 309.

[REY 93] E. Reya, 'QCD 20 years later', Ed. P.H. Zerwas and H.A. Kastrup, World Scientific, 1993, Vol. 1, p. 272.

[ROE 90] N.A. Roe et al., (ASP-Collaboration) Phys. Rev. D41 (1990) 17; D.I. Diakonov and M.I. Eides, 'Elementary Particle Physics', Lecture at XVI LIYaP Winter School, LIYaP, 1981, p. 123.

[ROS 90] G.G. Ross and R.G. Roberts, Preprint RAL-90-062, August 1990; unpublished.

[RYS 93] M.G. Ryskin, Phys. Lett. B319 (1993) 346.

[RYZ 89] Z. Ryzak, Phys. Lett. B217 (1989) 325; also private communication.

[SHO 92] G.M. Shore and G. Veneziano, Nucl. Phys. B381 (1992) 23.

[SHU 82] E.V. Shuryak and A.I. Vainshtein, Nucl. Phys. B201 (1982) 142.

[SRI 92] K. Sridhar and E. Leader, Phys. Lett. B295 (1992) 283.

[STR 92] M. Stratmann and W. Vogelsang, Phys. Lett. B295 (1992) 277.

[TOR 84] N. Törnqvist, P. Zenczykowski, Phys. Rev. D29 (1984) 2139.

['THO 76] G. 't Hooft, Phys. Rev. Lett. 37 (1976) 8; Phys. Rev. D14 (1976) 3432.

[VEN 79] G. Veneziano, Nucl. Phys. B159 (1979) 213.

[VEN 89] G. Veneziano, Mod. Phys. Lett. A4 (1989) 1605; G.M. Shore and G. Veneziano, Phys. Lett B244 (1990) 75.

[VEN 92] G. Veneziano and G. M Shore, Nucl. Phys. B381 (1992) 3.

[VOG 91] W. Vogelsang and A. Weber, Nucl. Phys. B362 (1991) 3.

[WA2 83] WA2 Group, Z. Phys. C21 (1983) 27; Particle Data Group, Phys. Lett. 111B (1982).

[WAN 77] S. Wandzura and F. Wilczek, Phys. Lett. B72 (1977) 195.

[WHI 90] L.W. Whitlow et al., Phys. Lett. B250 (1990) 193.

[WIN 92] R. Windmolders, Int. J. Mod. Phys. A7 (1992) 639.

[WOL 89] R.M. Woloshyn, Nucl. Phys. A496 (1989) 749.

[XIA 93] Xiangdong Ji, Nucl. Phys. B402 (1993) 217. 


\section{Figure captions}

Fig. 2.1 Feynman diagram for inelastic lepton-nucleon scattering: $\ell N \rightarrow \ell X$.

Fig. 2.2 Definition of the angles $\alpha, \beta, \theta, \varphi, \phi$ and $\Theta$.

Fig. 2.3 Further definition of the angles $\alpha, \theta$ and $\phi$.

Fig. 2.4 Spin configuration relevant to Eq. (2.1.39) for the measurement of $A_{\perp}$ with $\phi=0$.

Fig. 2.5 a) Lowest order Feynman diagrams for weak interaction contributions to $\ell N \rightarrow$ $\ell X$ and b) to $\ell^{\mp} N \rightarrow \nu(\bar{\nu}) X$.

Fig. 3.1 Predictions for $x g_{2}(x)$ and $x\left[g_{1}(x)+g_{2}(x)\right]$ based on Eq. (3.3.11) [JAC 89].

Fig. 3.2 Prediction for $g_{2}(x)$ in the bag model [JAF 91]. Dotted line $=$ twist-2 contribution; dashed $=$ twist-3; solid $=$ sum.

Fig. 3.3 Interaction of hard photon with quark.

Fig. 4.1 EMC (•) [ASH 89] and SLAC-Yale (o) [ALG 78; BAU 83] data on $A_{1}(x)$.

Fig. 4.2 EMC data on $x g_{1}^{p}(x)$. Also shown is the extrapolation to $x=0$ of the integral of $g_{1}^{p}(x)$ (see text).

Fig. 4.3 Comparison of the new SMC data on $g_{1}^{p}(x)$ at $\left\langle Q^{2}\right\rangle=10(\mathrm{GeV} / \mathrm{c})^{2}$ [ADA 94] with EMC data.

Fig. 4.4 Behaviour of $I_{p}\left(Q^{2}\right)$ (curve 1) and $I_{p}\left(Q^{2}\right)-I_{n}\left(Q^{2}\right)$ (curve 2) at large $Q^{2}$ and as given by the GDH sum rule at $Q^{2}=0$ (cross and triangle respectively, from [ANS 89]).

Fig. 4.5 The resonance contribution to $I\left(Q^{2}\right)$ for proton, neutron and their difference (from [BUR 93]).

Fig. 4.6 SMC data on the deuteron asymmetry $A_{1}^{d}$ at $Q^{2}=4.6(\mathrm{GeV} / \mathrm{c})^{2}$ (from $[\mathrm{ADE}$ $93])$.

Fig. 4.7 SMC data on $x g_{1}^{d}(x)$ at $Q^{2}=4.6(\mathrm{GeV} / \mathrm{c})^{2}$ (from [ADE 93]).

Fig. 4.8 E142 data on the neutron asymmetry $A_{1}^{n}(x)$ and on $g_{1}^{n}(x)$ at $Q^{2}=2(\mathrm{GeV} / \mathrm{c})^{2}$. See text (from [ANT 93]).

Fig. 4.9 $g_{1}^{p}\left(x, Q^{2}\right)$ at various $Q^{2}$ as extracted from the deuterium and helium-3 data by [ELL 93] (see text). Continuous curve, $Q^{2}=2(\mathrm{GeV} / \mathrm{c})^{2}$; dotted, $Q^{2}=4.6$; dotdashed, $Q^{2}=10.7$.

Fig. 4.10 $g_{1}^{n}\left(x, Q^{2}\right)$ as extracted by [ELL 93] (see text). Continuous curve, $Q^{2}=2$ $(\mathrm{GeV} / \mathrm{c})^{2} ;$ dotted, $Q^{2}=4.6$, dot-dashed, $Q^{2}=10.7$.

Fig. 6.1 Triangle diagram giving rise to the axial anomaly.

Fig. 6.2 Diagram giving rise to renormalization of axial anomaly.

Fig. 6.3 Diagram giving rise to renormalization of the axial current.

Fig. 8.1 Born diagram for $\gamma^{*} q \rightarrow q$.

Fig. 8.2 Lowest order diagram for $\gamma^{*} g \rightarrow q \bar{q}$. 
Fig. 8.3 Dependence of $d \Delta \sigma^{\gamma^{*} q} / d k_{T}^{2}$ on $k_{T}$ for several choices of the quark mass.

Fig. 8.4 Lowest order diagram for $\gamma^{*} q \rightarrow g q$.

Fig. 8.5 Result of fitting $g_{1}^{p}(x)$ with $\Delta s=0$, and $\Delta g$ constrained by the unpolarized glue. The solid line corresponds to $F / D=0.63$ and the dashed line to $F / D=0.55$.

Fig. 8.6 Result of fitting $g_{1}^{p}(x)$ with $\Delta s \neq 0$, and $\Delta g$ constrained by the unpolarized glue. The solid line corresponds to $F / D=0.63$ and the dashed line to $F / D=0.55$.

Fig. 8.7 $Z_{0}$ exchange contribution to elastic $\nu p \rightarrow \nu p$.

Fig. 8.8 Anomaly contribution to elastic $\nu p \rightarrow \nu p$.

Fig. 9.1 The QCD potential $V(X, Y, \ldots)$ as a function of $X$.

Fig. 9.2 Graphical representation of the non-perturbative coupling of the bare axial ghost to the current $\widetilde{K}_{\mu}$.

Fig. 9.3 Graphical representation of the coupling between the axial ghost and the field $\eta_{0}(x)$.

Fig. 9.4 Graphical representation of matrix elements a) $\left\langle T\left[G_{\mu} G_{\nu}\right]\right\rangle$ and b) $\left\langle T\left[G_{\mu} \eta^{\prime}\right]\right\rangle$.

Fig. 9.5 Graphical representation of matrix element $\left\langle P^{\prime}, \lambda^{\prime}\left|\widetilde{K}_{\mu}(0)\right| P, \lambda\right\rangle$.

Fig. 9.6 Graphical representation of interaction of $\eta^{0}$ and ghost $G_{\mu}^{0}$ with nucleon.

Fig. 10.1 Separation of $\gamma^{*}+$ hadron $\rightarrow X$ into soft and hard parts.

Fig. 10.2 a) Born diagram for $\gamma^{*} q \rightarrow \gamma^{*} q$ and b) the crossed version.

Fig. 10.3 DIS interaction involving quark-gluon correlation.

Fig. 11.1 Conventional Born mechanism for large $p_{T}$ Drell-Yan pair production.

Fig. C.1 Born diagram for $E^{\mu \rho \nu}$. There is also a crossed diagram analogous to Fig. 10.2b. 
This figure "fig1-1.png" is available in "png" format from: http://arxiv.org/ps/hep-ph/9501369v2 
This figure "fig2-1.png" is available in "png" format from: http://arxiv.org/ps/hep-ph/9501369v2 
This figure "fig3-1.png" is available in "png" format from: http://arxiv.org/ps/hep-ph/9501369v2 
This figure "fig4-1.png" is available in "png" format from: http://arxiv.org/ps/hep-ph/9501369v2 
This figure "fig5-1.png" is available in "png" format from: http://arxiv.org/ps/hep-ph/9501369v2 
This figure "fig6-1.png" is available in "png" format from: http://arxiv.org/ps/hep-ph/9501369v2 
This figure "fig7-1.png" is available in "png" format from: http://arxiv.org/ps/hep-ph/9501369v2 
This figure "fig1-2.png" is available in "png" format from: http://arxiv.org/ps/hep-ph/9501369v2 
This figure "fig2-2.png" is available in "png" format from: http://arxiv.org/ps/hep-ph/9501369v2 
This figure "fig3-2.png" is available in "png" format from: http://arxiv.org/ps/hep-ph/9501369v2 
This figure "fig4-2.png" is available in "png" format from: http://arxiv.org/ps/hep-ph/9501369v2 
This figure "fig5-2.png" is available in "png" format from: http://arxiv.org/ps/hep-ph/9501369v2 
This figure "fig6-2.png" is available in "png" format from: http://arxiv.org/ps/hep-ph/9501369v2 
This figure "fig7-2.png" is available in "png" format from: http://arxiv.org/ps/hep-ph/9501369v2 
This figure "fig1-3.png" is available in "png" format from: http://arxiv.org/ps/hep-ph/9501369v2 
This figure "fig2-3.png" is available in "png" format from: http://arxiv.org/ps/hep-ph/9501369v2 
This figure "fig3-3.png" is available in "png" format from: http://arxiv.org/ps/hep-ph/9501369v2 
This figure "fig4-3.png" is available in "png" format from: http://arxiv.org/ps/hep-ph/9501369v2 
This figure "fig6-3.png" is available in "png" format from: http://arxiv.org/ps/hep-ph/9501369v2 
This figure "fig7-3.png" is available in "png" format from: http://arxiv.org/ps/hep-ph/9501369v2 
This figure "fig3-4.png" is available in "png" format from: http://arxiv.org/ps/hep-ph/9501369v2 
This figure "fig4-4.png" is available in "png" format from: http://arxiv.org/ps/hep-ph/9501369v2 
This figure "fig5-4.png" is available in "png" format from: http://arxiv.org/ps/hep-ph/9501369v2 
This figure "fig6-4.png" is available in "png" format from: http://arxiv.org/ps/hep-ph/9501369v2 
This figure "fig7-4.png" is available in "png" format from: http://arxiv.org/ps/hep-ph/9501369v2 
This figure "fig3-5.png" is available in "png" format from: http://arxiv.org/ps/hep-ph/9501369v2 
This figure "fig3-6.png" is available in "png" format from: http://arxiv.org/ps/hep-ph/9501369v2 
This figure "fig3-7.png" is available in "png" format from: http://arxiv.org/ps/hep-ph/9501369v2 
This figure "fig3-8.png" is available in "png" format from: http://arxiv.org/ps/hep-ph/9501369v2 
This figure "fig3-9.png" is available in "png" format from: http://arxiv.org/ps/hep-ph/9501369v2 
This figure "fig3-10.png" is available in "png" format from: http://arxiv.org/ps/hep-ph/9501369v2 
This figure "fig3-11.png" is available in "png" format from: http://arxiv.org/ps/hep-ph/9501369v2 
This figure "fig3-12.png" is available in "png" format from: http://arxiv.org/ps/hep-ph/9501369v2 
This figure "fig3-13.png" is available in "png" format from: http://arxiv.org/ps/hep-ph/9501369v2 
This figure "fig3-14.png" is available in "png" format from: http://arxiv.org/ps/hep-ph/9501369v2 
This figure "fig3-15.png" is available in "png" format from: http://arxiv.org/ps/hep-ph/9501369v2 
This figure "fig3-16.png" is available in "png" format from: http://arxiv.org/ps/hep-ph/9501369v2 
This figure "fig3-17.png" is available in "png" format from: http://arxiv.org/ps/hep-ph/9501369v2 
This figure "fig3-18.png" is available in "png" format from: http://arxiv.org/ps/hep-ph/9501369v2 
This figure "fig3-19.png" is available in "png" format from: http://arxiv.org/ps/hep-ph/9501369v2 
This figure "fig3-20.png" is available in "png" format from: http://arxiv.org/ps/hep-ph/9501369v2 
This figure "fig3-21.png" is available in "png" format from: http://arxiv.org/ps/hep-ph/9501369v2 
This figure "fig3-22.png" is available in "png" format from: http://arxiv.org/ps/hep-ph/9501369v2 